\title{
New In-Pile
}

Instrumentation to

Support Fuel Cycle

Research and

Development
J. Rempe
H. MacLean
R. Schley
D. Hurley
J. Daw
S. Taylor
J. Smith
J. Svoboda
D. Kotter
D. Knudson
S. C. Wilkins
M. Guers
L. Bond
L. Ott
J. McDuffee
E. Parma
G. Rochau

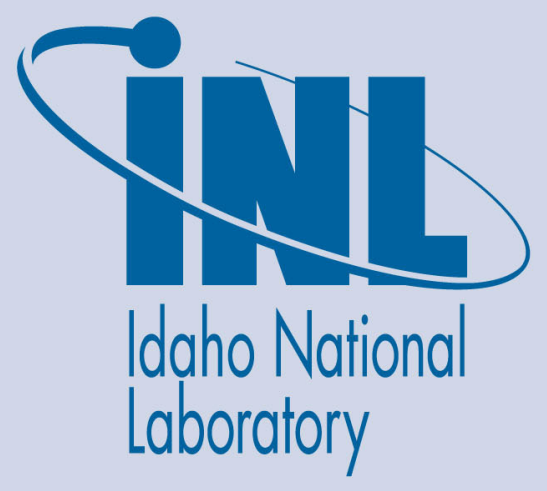

January 2011

The INL is a U.S. Department of Energy National Laboratory operated by Battelle Energy Alliance 
INL/EXT-10-19149

FCRD-FUEL-2011-000033

\title{
New In-Pile Instrumentation to Support Fuel Cycle Research and Development
}

\author{
J. Rempe \\ H. MacLean \\ R. Schley \\ D. Hurley \\ J. Daw \\ S. Taylor \\ J. Smith \\ J. Svoboda \\ D. Kotter \\ D. Knudson \\ S. C. Wilkins \\ M. Guers \\ L. Bond \\ L. Ott \\ J. McDuffee \\ E. Parma \\ G. Rochau
}

January 2011

\author{
Idaho National Laboratory \\ Fuel Cycle Research \& Development \\ Advanced Fuels Campaign \\ Idaho Falls, Idaho 83415 \\ http://www.inl.qov \\ Prepared for the \\ U.S. Department of Energy \\ Under DOE Idaho Operations Office \\ Contract DE-AC07-05ID14517
}




\section{DISCLAIMER}

This information was prepared as an account of work sponsored by an agency of the U.S. Government. Neither the U.S. Government nor any agency thereof, nor any of their employees, makes any warranty, expressed or implied, or assumes any legal liability or responsibility for the accuracy, completeness, or usefulness, of any information, apparatus, product, or process disclosed, or represents that its use would not infringe privately owned rights. References herein to any specific commercial product, process, or service by trade name, trade mark, manufacturer, or otherwise, does not necessarily constitute or imply its endorsement, recommendation, or favoring by the U.S. Government or any agency thereof. The views and opinions of authors expressed herein do not necessarily state or reflect those of the U.S. Government or any agency thereof. 


\section{SUMMARY}

New and enhanced nuclear fuels are a key enabler for new and improved reactor technologies. For example, the goals of the next generation nuclear plant (NGNP) will not be met without irradiations successfully demonstrating the safety and reliability of new fuels. Likewise, fuel reliability has become paramount in ensuring the competitiveness of nuclear power plants. Recently, the Office of Nuclear Energy in the Department of Energy (DOE-NE) launched a new direction in fuel research and development that emphasizes an approach relying on first principle models to develop optimized fuel designs that offer significant improvements over current fuels. To facilitate this approach, high fidelity, real-time, data are essential for characterizing the performance of new fuels during irradiation testing. A three-year strategic research program has been initiated for developing the required test vehicles with sensors of unprecedented accuracy and resolution for obtaining the data needed to characterize three-dimensional changes in fuel microstructure during irradiation testing. When implemented, this strategy will yield test capsule designs that are instrumented with new sensor technologies for irradiations at facilities primarily relied upon by the Fuel Cycle Research and Development (FCR\&D) program, the Advanced Test Reactor (ATR) and the High Flux Isotope Reactor (HFIR). Prior laboratory testing, and as needed, irradiation testing of sensors in these capsules will have been completed to give sufficient confidence that the irradiation tests will yield the required data.

From the onset of this instrumentation development effort, it was recognized that obtaining these sensors must draw upon the expertise of a wide-range of organizations not currently supporting nuclear fuels research. Hence, a draft version of this document was developed to provide necessary background information related to fuel irradiation testing, desired parameters for detection, and an overview of currently available in-pile instrumentation. Then, a workshop was held in which U.S. and foreign experts from fuels, irradiation, and instrumentation fields participated. Prior to this workshop, copies of a draft version of this document were distributed to participants to stimulate expert interactions at this meeting. During the workshop, candidate sensor technologies identified in this document were discussed and ranked by the experts using agreed upon criteria. The final version of this document describes the consensus reached during the workshop with respect to recommendations for the path forward for accomplishing the goals of this research program.

Based on the activities completed to develop this strategic plan, it is recommended that the FCR\&D instrumentation development program be initiated as a three year program that includes the following three tasks:

- Ultrasonics-Based Evaluations - In this task, laboratory evaluations and necessary irradiations will be completed to demonstrate the viability of this technology for in-pile applications. Specifically, tasks should be identified for obtaining the required signal processing upgrades, demonstrating the viability of ultrasonics to detect the parameters of interest under various anticipated test conditions (e.g., inert gas and sodium) with the required accuracy, and for developing test capsule(s) in which the proposed sensors could be deployed at the ATR and HFIR.

- Fiber Optics-Based Evaluations - In this task, laboratory evaluations and necessary irradiations will be completed to demonstrate the viability of this technology for in-pile applications. Specifically, tasks should be identified for obtaining the required signal processing, demonstrating the viability of fiber optics to detect the parameters of interest under various anticipated test conditions (e.g., inert gas and sodium) with the required accuracy (note that suitable coatings/sheaths must be identified for sodium applications and temperature limitations must be quantified). In addition, test capsule(s) designs will be developed in which the proposed sensors could be deployed at the ATR and HFIR.

- Upgrade US MTR In-Pile Instrumentation - In this task, laboratory evaluations and necessary irradiations will be completed to demonstrate the viability of enhanced detectors under anticipated ATR and 
HFIR irradiation conditions. Candidate sensors for enhancement include sensors for elongation and diameter evaluations, flux detectors, and thermal conductivity probes.

For each task, detailed project plans will be developed with a schedule identifying what can be accomplished within a three-year time period. Plans will also identify 'off-ramps' at key decision points where the viability of the technology could be assessed prior to further research. In addition, project plans will identify collaborations to ensure that research will take advantage of existing expertise for each technology. 


\section{CONTENTS}

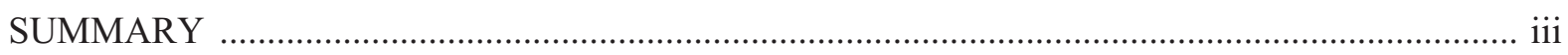

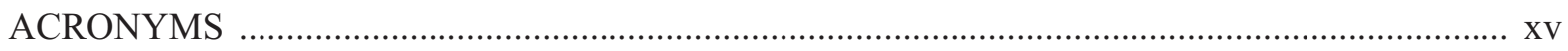

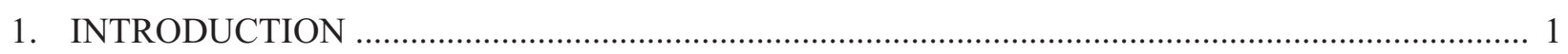

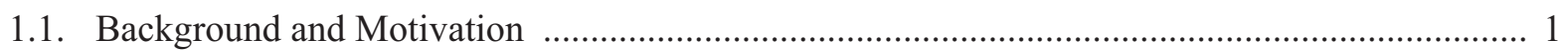

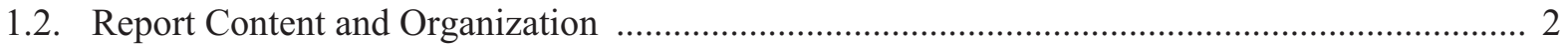

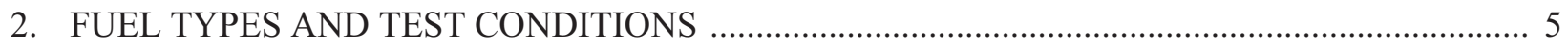

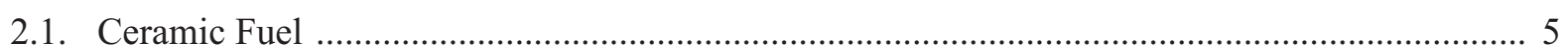

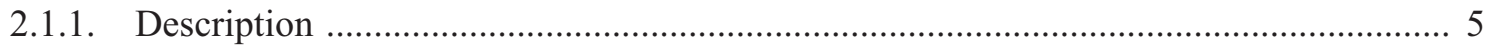

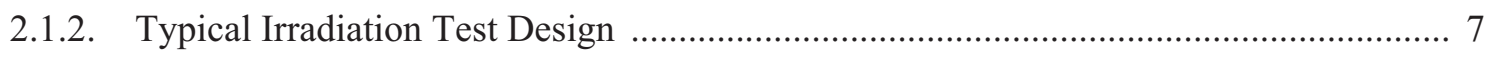

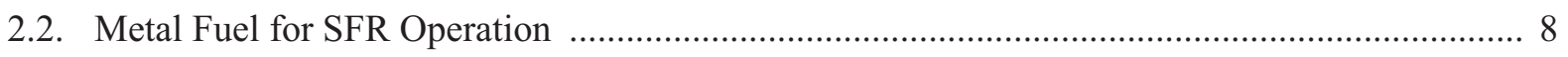

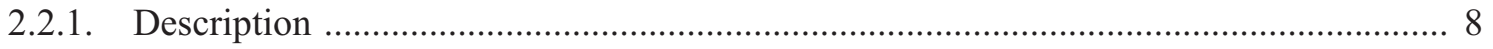

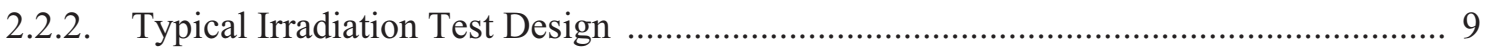

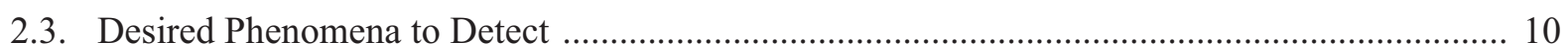

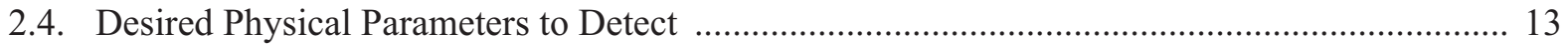

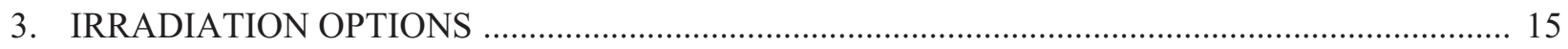

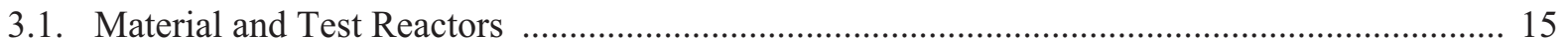

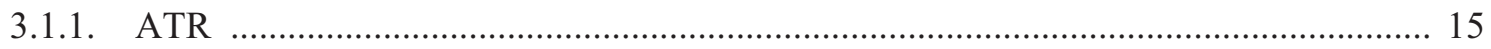

3.1.1.1. Reactor Design and Characteristics ..................................................... 15

3.1.1.2. Test Configurations and Conditions ..................................................... 15

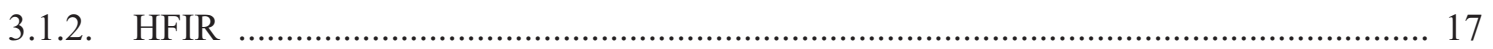

3.1.2.1. Reactor Design and Characteristics .................................................... 17

3.1.2.2. Test Configurations and Conditions ...................................................... 19

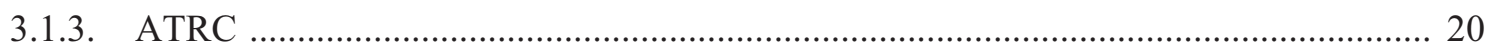

3.1.3.1. Reactor Design and Characteristics ..................................................... 20

3.1.3.2. Test Configurations and Conditions .................................................... 21

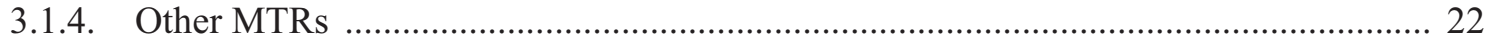

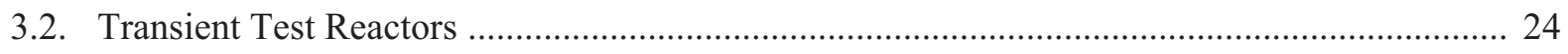

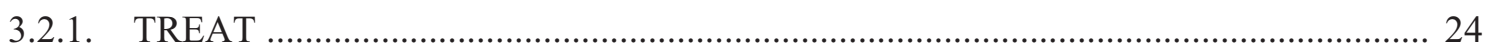

3.2.1.1. Reactor Design and Characteristics ....................................................... 24

3.2.1.2. Test Configurations and Conditions …….............................................. 25

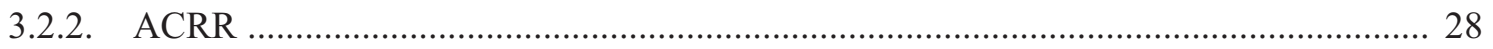

3.2.2.1. Reactor Design and Characteristics .................................................... 28

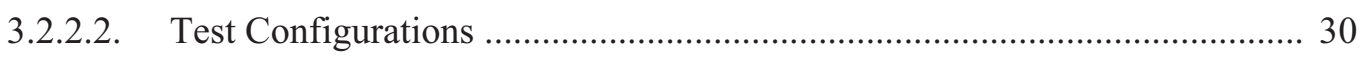

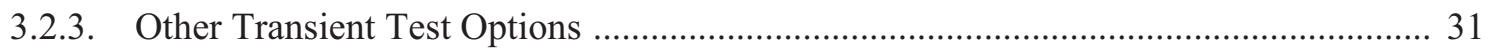

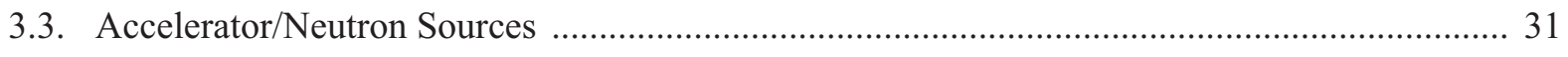

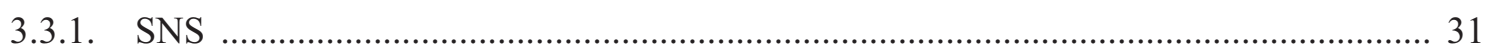

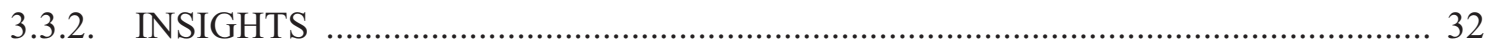




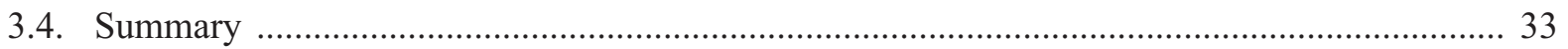

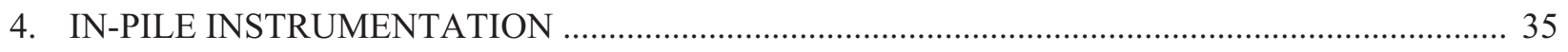

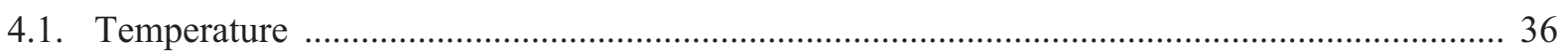

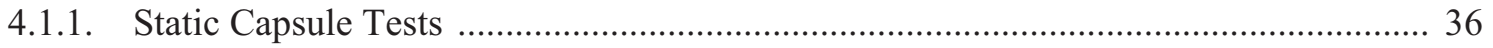

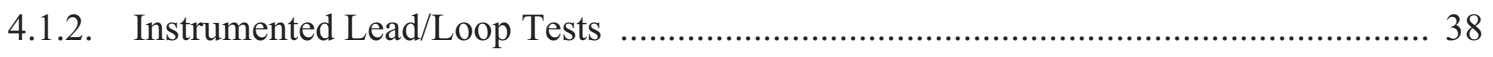

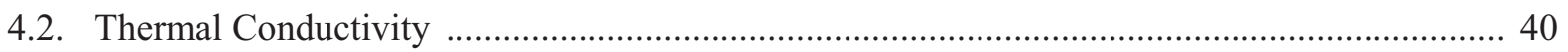

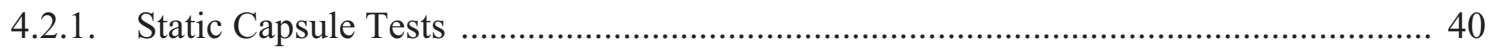

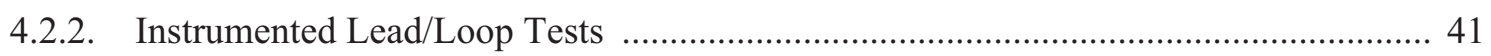

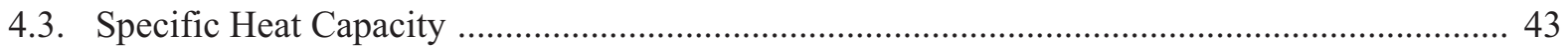

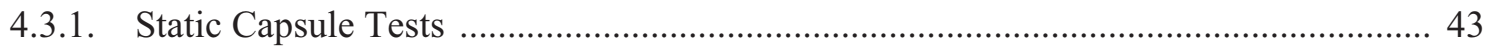

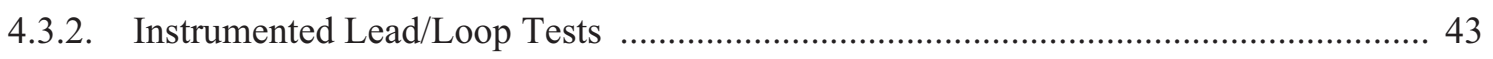

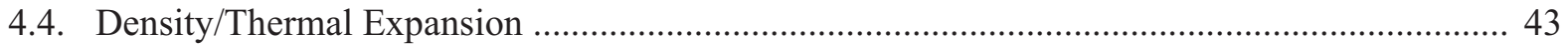

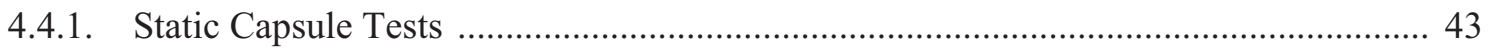

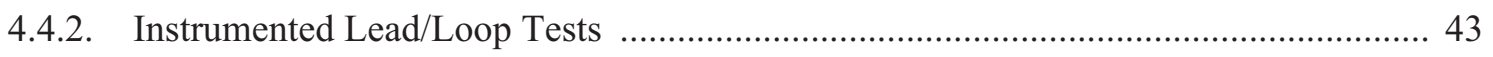

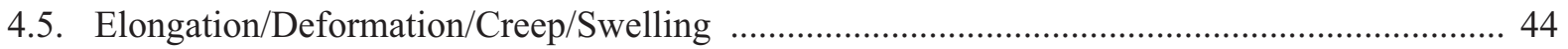

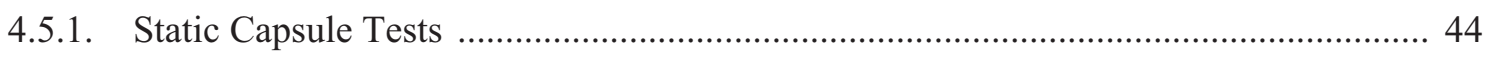

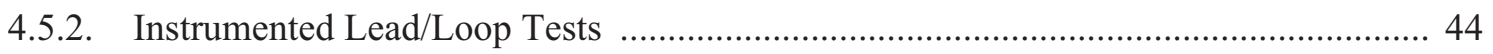

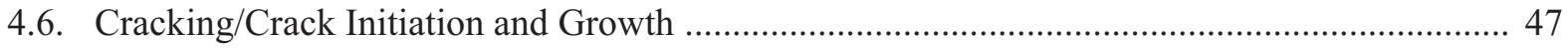

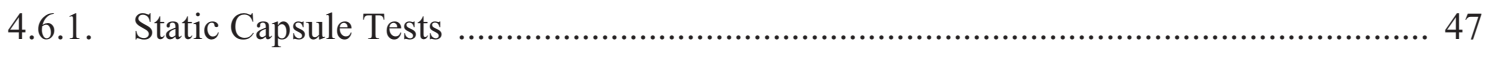

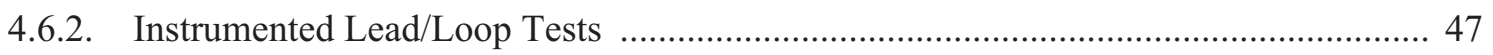

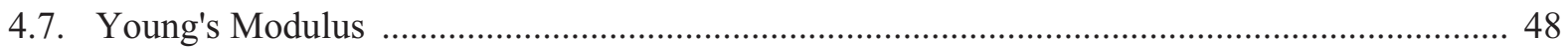

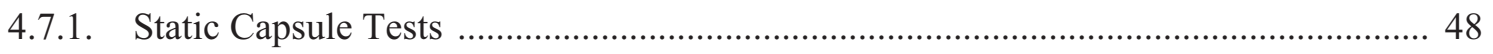

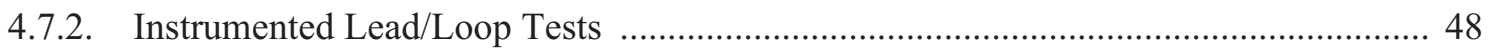

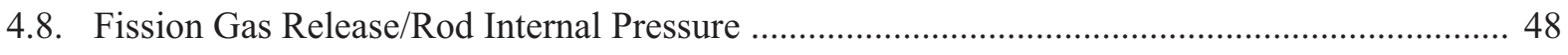

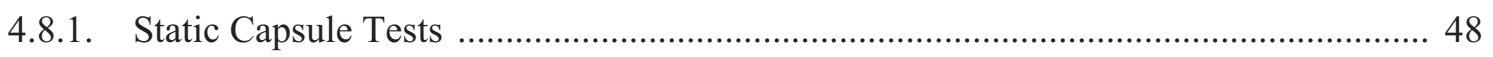

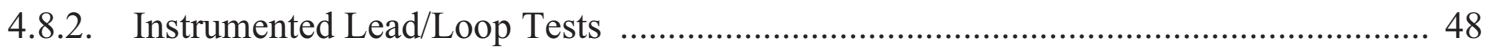

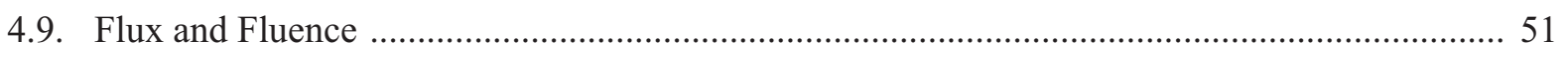

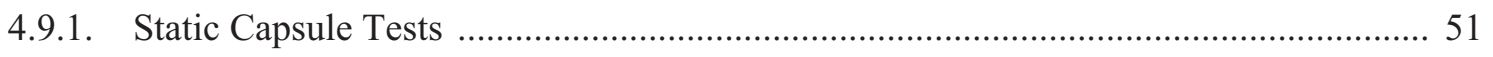

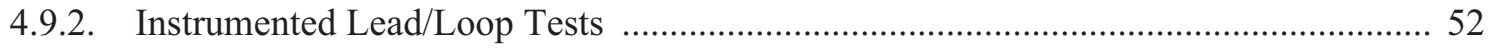

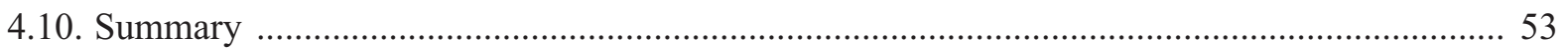

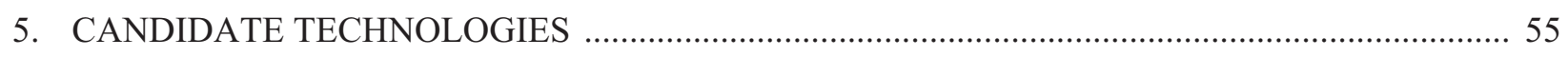

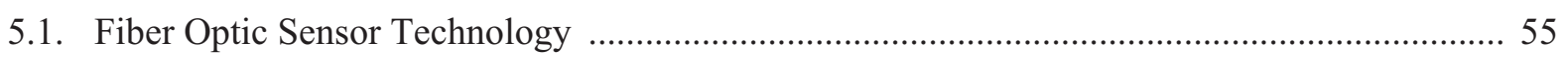

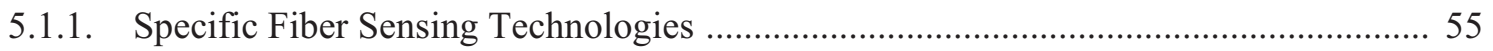

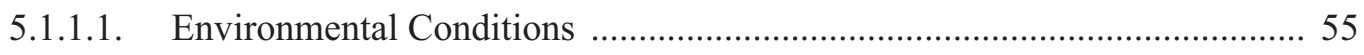

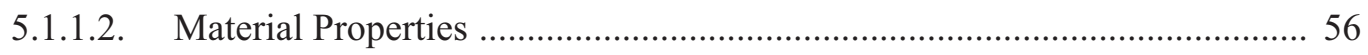

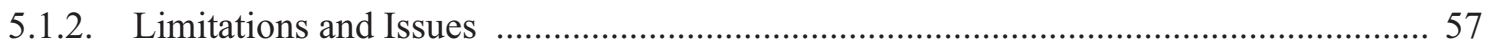

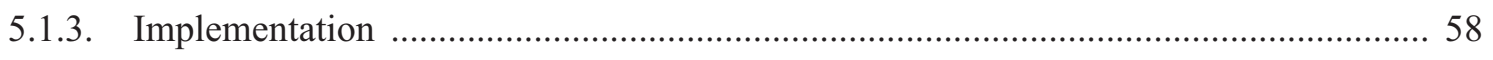

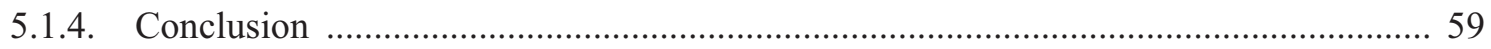




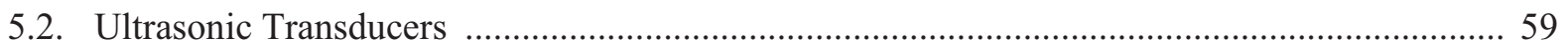

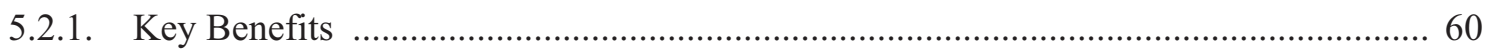

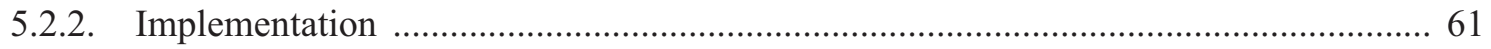

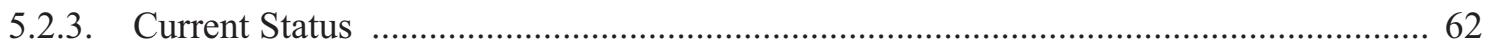

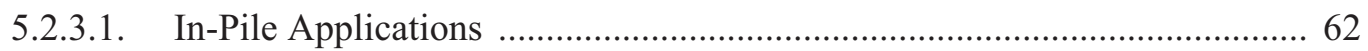

5.2.3.2. Liquid-Metal Reactor Applications ........................................................ 62

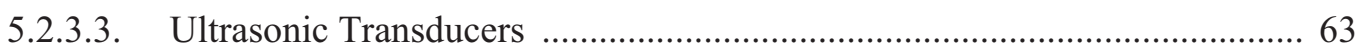

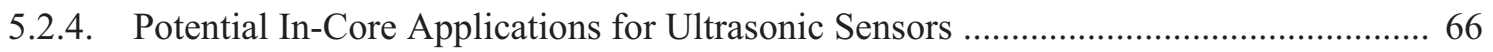

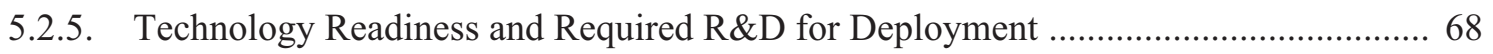

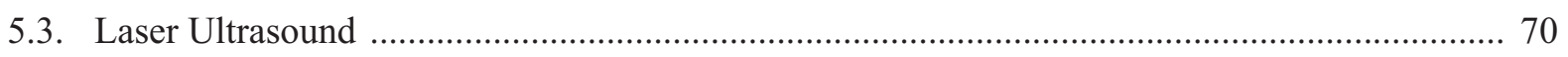

5.3.1. Laser-based Resonant Ultrasound Spectroscopy .................................................... 71

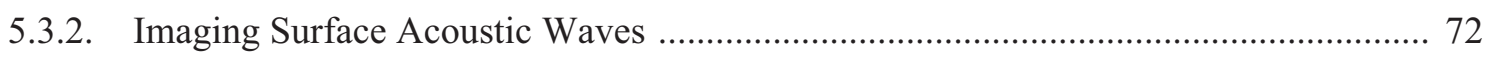

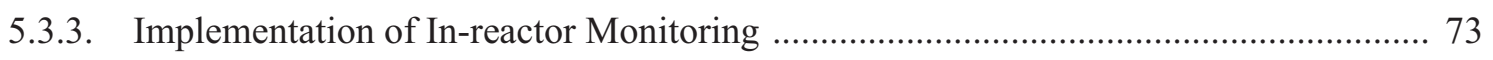

5.3.4. Implementation into an Irradiation Test Capsule ..................................................... 73

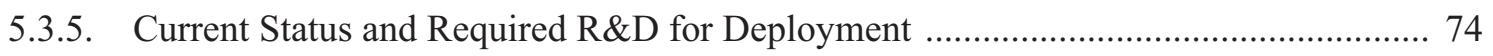

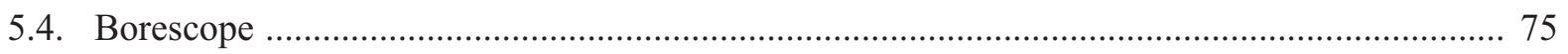

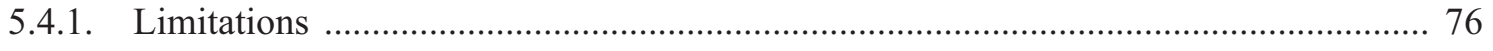

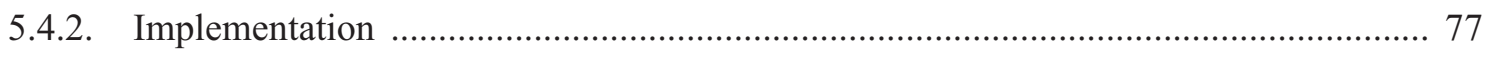

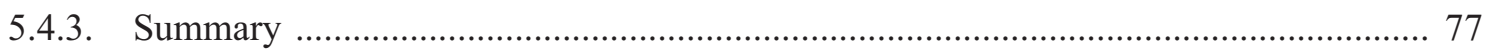

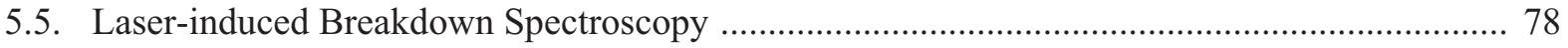

5.5.1. Implementation into an Irradiation Test Capsule .................................................... 79

5.5.2. Current Status and Required R\&D for Deployment .............................................. 81

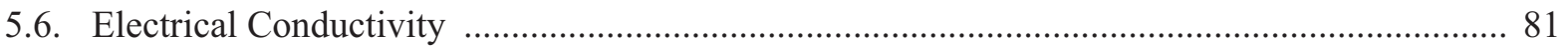

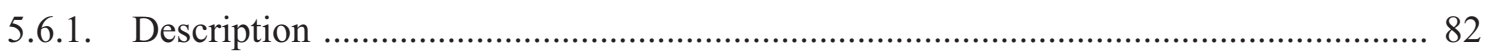

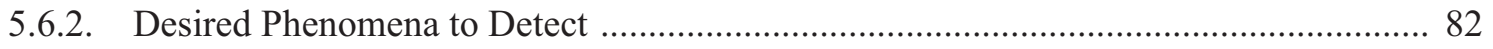

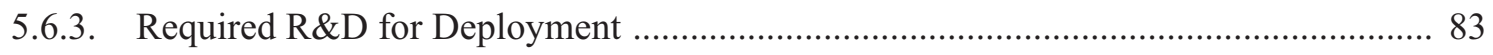

5.6.4. Desired Physical Parameters to be Detected ............................................................ 84

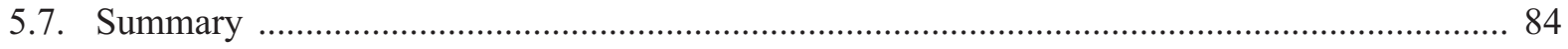

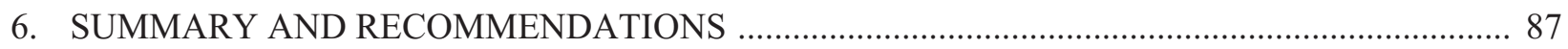

6.1. Test Conditions and Current In-Pile Instrumentation ......................................................... 87

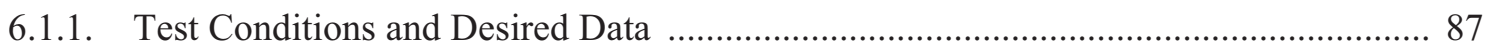

6.1.2. Currently Available Instrumentation to Provide Required Data ................................ 88

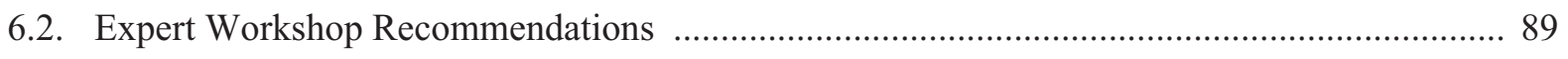

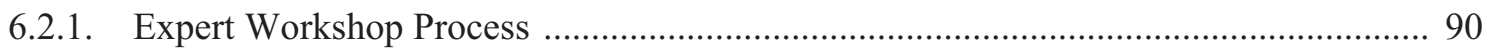

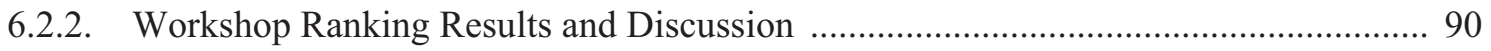

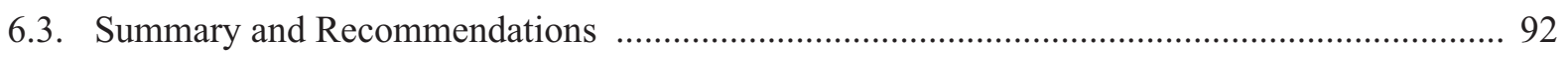

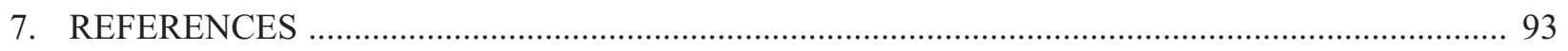

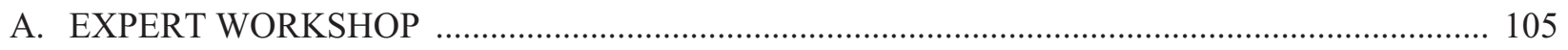




\section{FIGURES}

1. Schematic of LWR-2 fuel rodlet and capsule assembly for ATR irradiation. ............................... 6

2. Top view of LWR-2 test assembly for ATR irradiation. ......................................................... 6

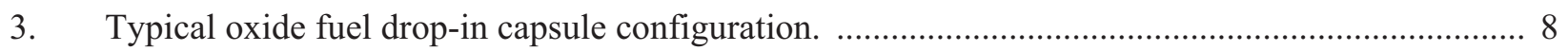

4. Typical metallic fuel drop-in capsule configuration. .............................................................. 9

5. Examples of constituent redistribution in metallic fast reactor fuels. ......................................... 10

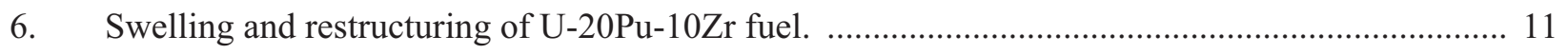

7. Fission gas release for fast reactor metallic fuel compositions. ................................................ 11

8. Examples of catastrophic fuel failure (left) and fuel restructuring (right). ................................ 12

9. ATR core cross section showing irradiation locations. ............................................................ 16

10. Schematic diagrams illustrating ATR irradiation locations. ..................................................... 16

11. Schematic of HFIR reactor core and beryllium reflector. ....................................................... 18

12. Cross section through HFIR pressure vessel. ........................................................................... 18

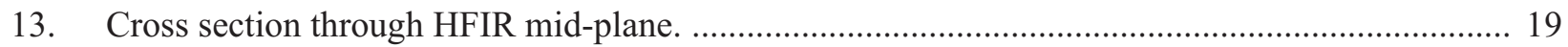

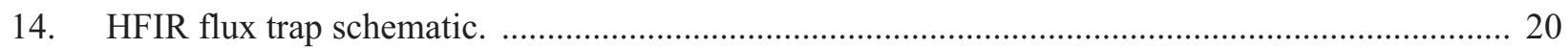

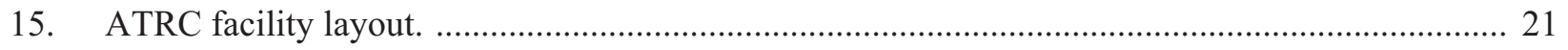

16. Sample /sensor positioning equipment and insertion locations at ATRC. ................................. 22

17. TREAT (a) facility schematic and (b) reactor shield (the Hodoscope is shown on the north face of the reactor, and a cask is shown on the radiography stand on the west face). .................. 25

18. Representative TREAT experimental loops. ............................................................................ 26

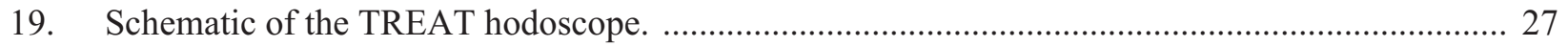

20. Example of analyzed TREAT hodoscope data (fuel motion with time). .................................... 28

21. ACRR and FREC-II operating at $2 \mathrm{MW}$ steady-state power. .................................................... 29

22. Cross-sectional view of the ACRR as modeled in MCNP. ..................................................... 30

23. Experiment configuration for a single or multiple fuel rodlets. ................................................... 31

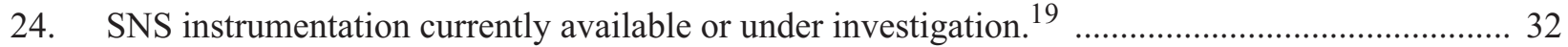

25. INSIGHTS conceptual design showing neutron source (yellow) surrounded by lead

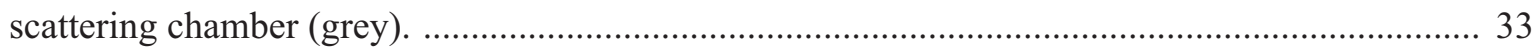

26. Setup to anneal and measure electrical resistivity of $\mathrm{SiC}$ temperature monitors. ........................ 37

27. Electrical resistivity measurement comparison on $\mathrm{SiC}$ monitors irradiated at $300^{\circ} \mathrm{C}$................ 37

28. Representative thermocouple response in INL $1200{ }^{\circ} \mathrm{C}$ tests. ................................................. 38

29. HTIR-TCs installed in AGR-1 test capsule and representative HTIR-TC and Type N data

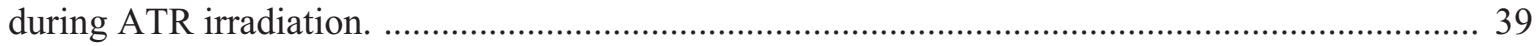

30. Schematic diagram of Expansion Thermometer (ET) used by IFE/HRP. ................................ 39

31. A typical multi-sensor pulse/echo ultrasonic thermometry system and 'notched' sensor. .............. 40 
32. In-pile thermal conductivity methods under evaluation (see text for variable definitions). .......... 41

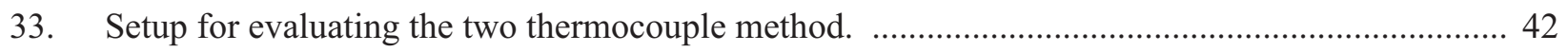

34. Setup for evaluating transient hot wire needle probe. .................................................................. 43

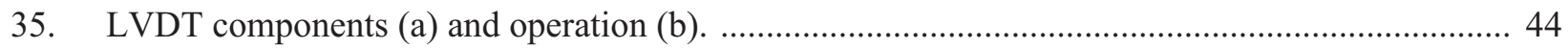

36. IFE/HRP test setups for detecting (a) cladding elongation; and (b) fuel elongation. ................... 45

37. HRP fuel pellet cladding interaction/crud deposition test rig (a) with diameter gauge (b). ........... 46

38. Tensile test module irradiated in the BR-2 ( $a$ and $b$ ) with schematic of tensile test module (c) that includes: (1) gas line, (2) pneumatic loading unit, (3) firm specimen fixing point, (4) specimen, (5) movable specimen fixing point, (6) LVDT plunger and (7) LVDT holder. ............ 47

39. Creep test rig positioned in autoclave for testing. ........................................................................ 47

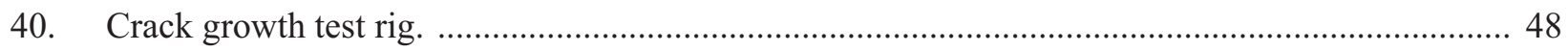

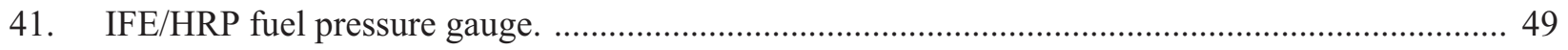

42. CEA counter-pressure sensor for measuring fission gas release. ............................................. 49

43. Setup for monitoring gas release during irradiation testing during the AGR-1 experiment

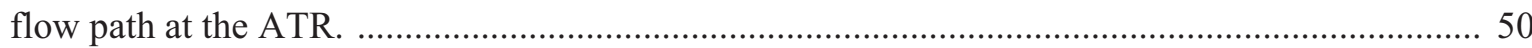

44. CEA fission gas release pressure and composition detection (a) sensor and (b) system

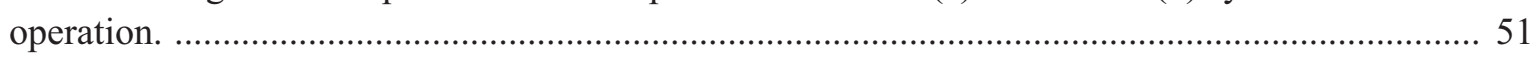

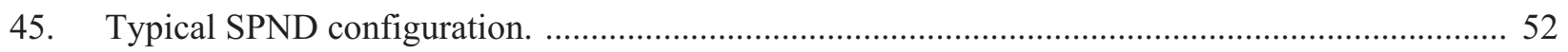

46. Representative miniature fission chamber configuration. ........................................................ 53

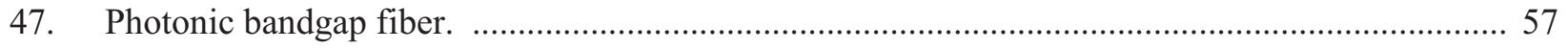

48. Schematic diagram of the fiber optic pressure boundary seal. ................................................. 58

49. Ultrasonic in-pile autoclave evaluations using (a) piezoelectric transducers or (b) magnetostrictive transducer on a creep specimen.

50. 'Stepped' array for in-situ scanning of fuel plates. ....................................................................... 61

51. Schematic illustrating PBF UT components and operation. ....................................................... 67

52. Potential capsule configuration for in-situ studies of ultrasonic transducer materials and wave propagation.

53. Resonant ultrasound used to provide in situ validation metric. The upper figure shows an electron backscatter diffraction (EBD) rendering of rolled and re-crystallized microstructures. The lower figure shows the change in the location of a single resonant peak during annealing. The data can be used to map the evolution of the elastic stiffness tensor during high temperature processing.

54. Results from investigation of surface acoustic wave propagation across an isolated grain boundary: (a) Image of surface acoustic phonon pulses propagating across a grain boundary (yellow line) in $\mathrm{Cu}$; (b) Electron backscatter micrograph of region of interest. 73

55. Conceptual sketch for implementing laser ultrasound in an irradiation test capsule. .................... 74

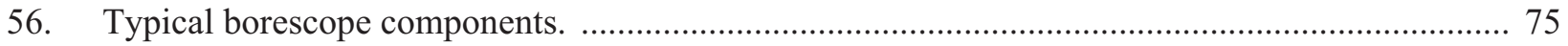


57. Fiberscope image illustrating individual pixel limitation on resolution. ${ }^{141}$................................ 76

58. Concept for implementing a borescope into an irradiation test capsule. ..................................... 77

59. Schematic of a LIBS system - Courtesy of US Army Research Laboratory. ${ }^{141}$.......................... 78

60. Broadband LIBS spectrum contains full information from 200 - $900 \mathrm{~nm}$. X-axis units are $\mathrm{nm}$, and $\mathrm{Y}$-axis units are relative intensity. ${ }^{142}$

61. Chromium concentration in steel alloys of differing composition is shown. Calibrations can be used to quantify concentrations, or chemometrics can be used to sort material. ${ }^{142}$ 80

62. Cross-section of circular DCF with offset core. ....................................................................... 81

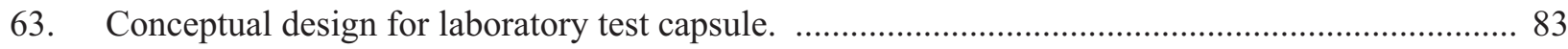




\section{TABLES}

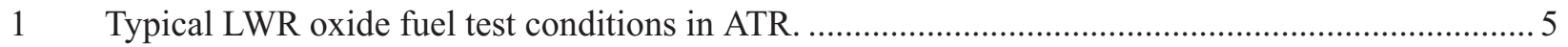

2 Previously-irradiated fast reactor ceramic fuel compositions. ................................................. 7

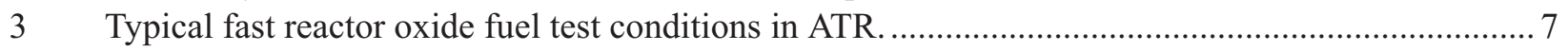

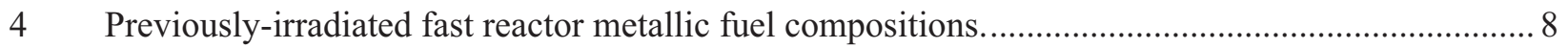

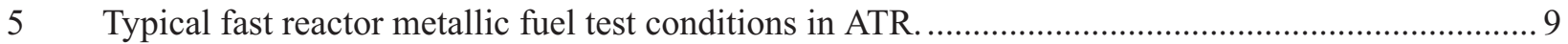

$6 \quad$ Summary of desired parameters for detection during fuel irradiation tests. ................................... 13

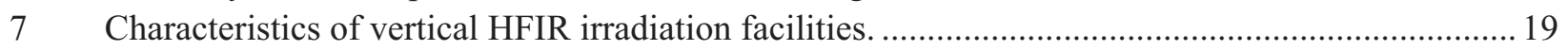

8 Comparison of design parameters in selected operating test reactors. ${ }^{18}$....................................... 23

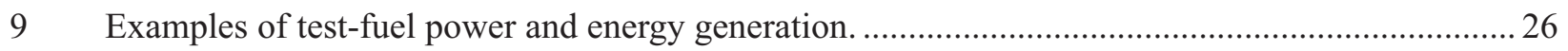

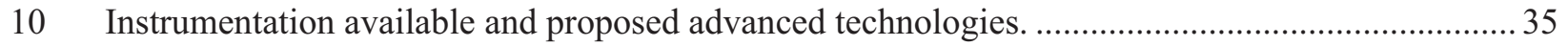

11 Summary of sensors deployed at or currently investigated for ATR and HFIR. ........................... 54

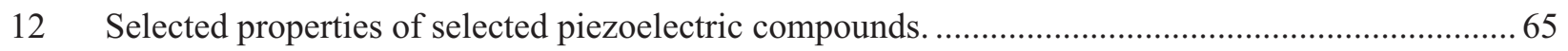

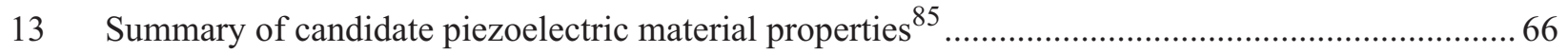

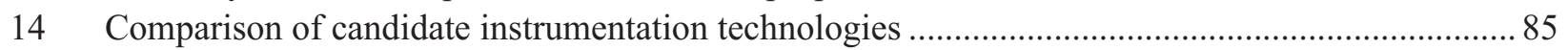

15 Summary of desired parameters for detection during fuel irradiation tests. .................................. 88

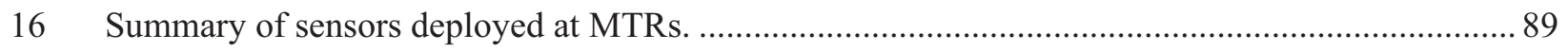

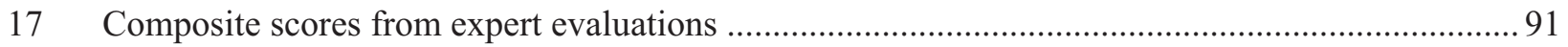




\section{ACRONYMS}

ACRR Annular Core Research Reactor

AFCI Advanced Fuel Cycle Initiative

ASTM American Society for Testing and Materials

ATR Advanced Test Reactor

ATRC ATR Critical

BWR Boiling Water Reactor

CCD Charge-coupled Device

CEA Commissariat à l'Énergie Atomique et aux Energies Alternatives

CT Compact Tension

DCF Double-clad Fiber

DCPD Direct Current Potential Drop

DOE Department of Energy

DOE-NE Office of Nuclear Energy in the Department of Energy

EBD Electron Backscatter Diffraction

EBR-II Experimental Breeder Reactor II

EFPI Extrinsic Fabry-Perot Interferometer

EPRI Electric Power Research Industry

ET Expansion Thermometer

FBG Fiber Bragg Grating

FCCI Fuel-Cladding Chemical Interaction

FCMI Fuel-Cladding Mechanical Interaction

FCR\&D Fuel Cycle Research and Development

FNDS Fast Neutron Detection System

FPMS Fission Product Monitoring System

FWHM Full width at half maximum (of pulse)

HBWR Halden Boiling Water Reactor

HEDL Hanford Engineering Development Laboratory

HFIR High-Flux Isotope Reactor

HSIS Hydraulic Shuttle Irradiation System

HTIR-TC High Temperature Irradiation Resistant ThermoCouple

HTTL High Temperature Test Laboratory

HTTR High Temperature Test Reactor

ID Inner Diameter

IFE/HRP Institute for Energy Technology at the Halden Reactor Project

INL Idaho National Laboratory

INSIGHTS Intense Neutron Spectra with Independent Gamma, Hydraulic and Temperature Separate-effects

JAEA Japan Atomic Energy

JHR Jules Horowitz Reactor 
KAERI Korea Atomic Energy Research Institute

LANL Los Alamos National Laboratory

LHGR Linear Heat Generation Rate

LIBS Laser-induced Breakdown Spectroscopy

LLNL Lawrence Livermore National Laboratory

LRUS Laser-based Resonant Ultrasound Spectroscopy

LVDT Linear Variable Differential Transformer

LWR Light Water Reactor

LWRS Light Water Reactor Sustainability

MCNP Monte Carlo N-Particle Transport Code

MFC Materials and Fuels Complex

MIT Massachusetts Institute of Technology

MITR Massachusetts Institute of Technology Nuclear Research Reactor

MOX Mixed OXide

MTR Material Test Reactor

NDE Nondestructive Evaluation

NDT Nondestructive Testing

NEWS Nonlinear Elastic Wave Spectroscopy

NGNP Next Generation Nuclear Plant

NSRR Nuclear Safety Research Reactor

NSUF National Scientific User Facility

OD Outer Diameter

ODS Oxide Dispersion Strengthened

OECD Organization for Economic Cooperation and Development

OFDR Optical Frequency Domain Reflectometry

ORNL Oak Ridge National Laboratory

OTDR Optical Time Domain Reflectometry

PBF Power Burst Facility

PBG Photonic Bandgap

PCF Power Coupling Factor

PIE Post-Irradiation Examination

PNNL Pacific Northwest National Laboratory

PWR Pressurized Water Reactor

PZT Lead Zirconium Titanate

RB* Large Removable Beryllium Facility

RIA Radiation Induced Attenuation

SFR Sodium Fast Reactor

SHM Structural Health Monitoring

SNL Sandia National Laboratory

SNS Spallation Neutron Source

SPNDs Self-powered Neutron Detectors 
THWM Transient Hot Wire Method

UT Ultrasonic Thermometer

TA-V Technical Area V

TED Total fission Energy Deposition

TEM Transmission Electron Microscope

TR Time Reversal

TREAT Transient REActor Test

TRISO Tri-structural Isotropic

TRU Transuranic

UK AEA United Kingdom Atomic Energy Authority

USU Utah State University

VXF Vertical Experiment Facility

WTP Waste Treatment Plant 


\section{Advanced Fuels Campaign New In-pile Instrumentation to Support Fuel Cycle Research and Development}

\section{INTRODUCTION}

New and enhanced nuclear fuels are a key enabler for new and improved reactor technologies. For example, the goals of the next generation nuclear plant (NGNP) will not be met without irradiations successfully demonstrating the safety and reliability of new fuels. Likewise, fuel reliability has become paramount in ensuring the competitiveness of nuclear power plants. Recently, the Office of Nuclear Energy in the Department of Energy (DOE-NE) launched a new direction in fuel research and development that emphasizes an approach relying on first principle models to develop optimized fuel designs that offer significant improvements over current fuels. To facilitate this approach, high fidelity, real-time, data are essential for characterizing the performance of new fuels during irradiation testing. A three-year strategic research program has been initiated for developing the required test vehicles with sensors of unprecedented accuracy and resolution for obtaining the data needed to characterize three-dimensional changes in fuel microstructure during irradiation testing. When implemented, this strategy will yield test capsule designs that are instrumented with new sensor technologies for irradiations at facilities primarily relied upon by the Fuel Cycle Research and Development (FCR\&D) program, the Advanced Test Reactor (ATR) at the Idaho National Laboratory (INL) and the High Flux Isotope Reactor (HFIR) at the Oak Ridge National Laboratory (ORNL). Prior laboratory testing, and as needed, irradiation testing of sensors deployed in these capsules will have been completed to give sufficient confidence that the irradiation tests will yield the required data.

From the onset of this instrumentation development effort, it was recognized that obtaining these sensors must draw upon the expertise of a wide-range of organizations not currently supporting nuclear fuels research. Hence, a draft version of this document was developed to provide necessary background information related to fuel irradiation testing, desired parameters for detection, and an overview of currently available in-pile instrumentation. Then, a workshop was held in which U.S. and foreign experts from fuels, irradiation, and instrumentation fields participated. Prior to this workshop, draft versions of this document were distributed to participants to stimulate expert interactions at this meeting. During the workshop, candidate sensor technologies identified in this document were discussed and ranked by experts using agreed upon criteria. The final version of this document describes the consensus reached during the workshop with respect to the path forward for accomplishing the goals of this research program.

\subsection{Background and Motivation}

As noted in Reference 1, DOE-NE wishes to "move beyond incremental improvement of existing reactor and fuel cycle technologies to achieve transformational advances in knowledge and application." DOE-NE2 defined several goals with respect to advanced sustainable fuel performance:

- Reduce transuranic production

- Perform fundamental analysis of fuel fabrication processes and their relation to fuel and cladding performance

- Evaluate very high burnup systems that require minimal or no chemical separations

To move beyond incremental improvement of existing reactor and fuel cycle technologies and achieve transformational advances in knowledge and application, science-based research is emphasized. 
The DOE-NE FC R\&D program places increased emphasis on advanced modeling and simulation tools to predict in-pile fuel behavior and to design innovative fuels that improve the performance of our existing commercial reactors and support new reactor designs. ${ }^{1-3}$ The goal of this FCR\&D Modeling and Simulation effort is to develop advanced tools that incorporate all of the relevant physics and chemistry, spanning phenomena from the macro-scale to the micro-structural level and over a large range of environmental variables, such as heat, pressure and radiation.

Improved modeling of the behavior of existing and advanced fuels faces several challenges. ${ }^{4}$ First, science-based models are needed to replace empirical correlations that are currently used to model major fuel behavior phenomena. However, these correlations were developed through existing databases of experimental data that are inadequate for developing science-based models. Second, there is a desire to predict fuel behavior with transuranics (TRU) added to the fuel. There are limited data for developing science-based models for these fuels. Last, there is the desire to develop models that can be used as a basis for extending fuel burnup to minimize waste. Those higher limits include higher burnups, linear powers, neutron fluences and operating temperatures. Again, there are limited data for developing and validating models for fuels exposed to these conditions. Though improved fuel modeling tools are potentially powerful vehicles of innovation and discovery, they have little value to the community without a dedicated accompanying effort to validate them.

Clearly, a new paradigm for instrumented in-pile testing is needed to achieve the transformational goals outlined by DOE-NE. Historically, fuel characterizations from irradiation tests are primarily done using a 'cook and look' approach at selected intervals. The time and labor to remove, examine, and return irradiated samples for each measurement makes this approach very expensive. In addition, such techniques provide limited data and may disturb the phenomena of interest. Recently, several research organizations affiliated with materials and test reactors have increased efforts to provide new in-pile sensors to support irradiation testing. ${ }^{5-11}$ However, none have started to develop the sensor technologies that would yield the data required to allow real-time observation of fuel microstructure and properties evolving during irradiation. The new data required to benchmark and validate models from DOE-NE's FCR\&D must be of unprecedented precision and resolution for early $(<24$ hours) and longer duration irradiations. Developing and deploying new sensors capable of delivering such high-precision, in-pile data is a daunting task, but key to implementing DOE-NE goals.

\subsection{Report Content and Organization}

This document outlines this strategic program to obtain the desired data during FCR\&D irradiation testing. It describes representative fuel irradiation tests, identifies the needs for data obtained from these tests, and provides recommendations for in-pile instrumentation that could be used to obtain such data. As noted previously, it was recognized that this effort must draw upon input from a wide-range of experts not currently supporting nuclear fuels research. Hence, a workshop was held to obtain this input and gain consensus on the approach recommended in this document. This document also reports results from this workshop.

The remainder of this report is organized into six sections. Section 2 describes representative fuel types and irradiation test conditions of interest to this program. Key phenomena identified in prior fuel performance evaluations are listed for which additional data are needed. Physical parameters that must be detected during irradiation tests are also identified with desired accuracies. Section 3 describes the ATR, HFIR, and other representative irradiation options in which new sensor technologies may be deployed in out-years to support FC R\&D irradiations. Section 4 provides an overview of in-pile instrumentation techniques currently used at ATR, HFIR, and other Materials and Test Reactors (MTRs) to obtain data from fuel irradiation tests. Section 5 identifies other instrumentation techniques, not currently used for in-pile applications, that could improve the fidelity of data obtained during fuel irradiations. Information in this section includes technologies described by experts at the workshop held to support this initiative. As noted 
previously, this workshop included researchers with nuclear and non-nuclear instrumentation expertise. Rankings provided by the experts on agreed-upon criteria are also provided in this section. Section 6 summarizes key information in this document. The proposed path forward for a research and development effort that could allow the FCR\&D program obtain the data required for developing and validating advanced models is also described. Section 7 lists references cited in this document. Appendix A provides additional details related to the expert workshop held to support this initiative. 


\section{FUEL TYPES AND TEST CONDITIONS}

To better understand the types of instrumentation that could be included in FCR\&D fuel irradiations, this section describes representative ceramic and metallic fuel types and irradiation test conditions that are currently of interest to this program. Key phenomena identified in prior fuel performance evaluations are identified for which additional data are needed. Physical parameters that must be detected during irradiation tests are also listed with desired accuracies.

It should be noted that in order to detect the parameters of interest with the desired accuracy, it may be necessary to have 'simplified' experimental tests that are non-prototypic. For example, sensors requiring a 'line-of-sight' may be deployed on metal fuel in inert gas tests. However, in such cases, detailed evaluations are needed to insure that obtained data are prototypic.

Last, it should be noted that coated particle fuel, which is primarily used in gas reactors, are not discussed in this section. Coated particle fuel development and testing is currently funded through DOE efforts to develop the NGNP. However, sensors developed from this effort are expected to also benefit future NGNP irradiations.

\subsection{Ceramic Fuel}

\subsubsection{Description}

Ceramic fuels (including oxide, nitride, and carbide, for example) are of interest for both thermal and fast reactor applications. Oxide fuels are employed in the U.S. commercial light water reactor (LWR) industry. Advanced oxide fuel designs are being investigated within the Light Water Reactor Sustainability (LWRS) program and include advanced fuel compositions and cladding materials.

The LWRS Advanced LWR Nuclear Fuel Development Pathway is currently designing irradiation experiments of prototype rodlets made with $\mathrm{SiC}$-based cladding. The rodlets will use a thin zirconium liner bonded with a ceramic matrix composite $\mathrm{SiC}$ outer tube with end caps welded to the zirconium liner. Initial irradiation experiments will consist of unfueled rodlets with $\mathrm{UO}_{2}$ fuel to follow at varying enrichments (anticipated $5 \%$ to $8 \%$ ).

Typical operating conditions for oxide fuels in LWRs, which are similar to proposed ATR test conditions for LWRS tests, are listed in Table 1.

Table 1. Typical LWR oxide fuel test conditions in ATR.

\begin{tabular}{|l|c|}
\hline \multicolumn{1}{|c|}{ Parameter } & Typical Value \\
\hline Linear Heat Generation Rate (LHGR) & $200-330 \mathrm{~W} / \mathrm{cm}$ \\
Fuel Centerline Peak Temperature & $1400{ }^{\circ} \mathrm{C}$ \\
Capsule Assembly Peak Pressure & $800 \mathrm{psi}$ \\
Flux (thermal) & $10^{14} \mathrm{n}_{\mathrm{th}} \mathrm{cm}^{2}-\mathrm{s}$ \\
\hline
\end{tabular}

An example irradiation capsule configuration used previously in ATR to irradiate LWR MOX fuel is shown in Figures 1 and 2.

Ceramic fuels are also under consideration for use in fast reactors where they could be used to transmute transuranic elements from used nuclear fuel from LWRs. Typical fast reactor ceramic parameters are based on those used in previous fast reactors, and fuel dimensions are based on those used in the Experimental Breeder Reactor II (EBR-II) program. 


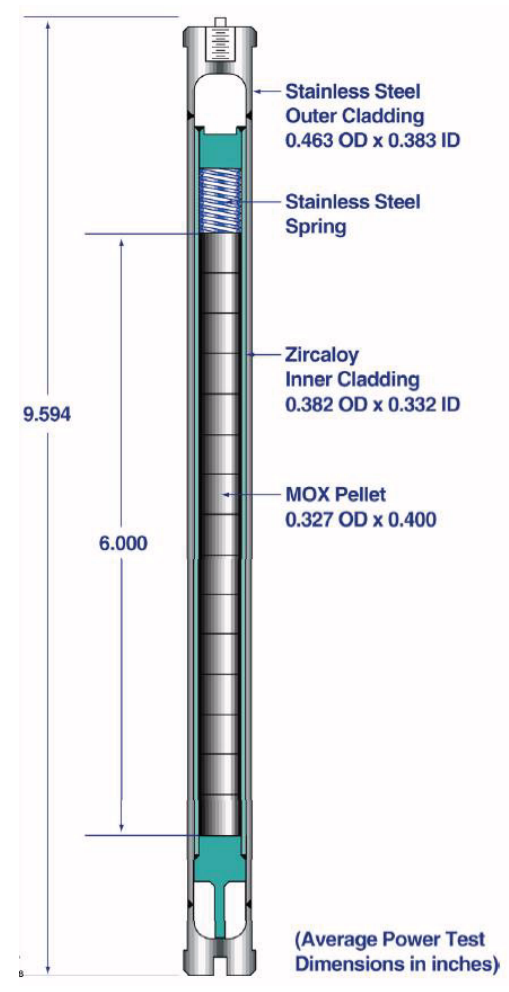

Figure 1. Schematic of LWR-2 fuel rodlet and capsule assembly for ATR irradiation.

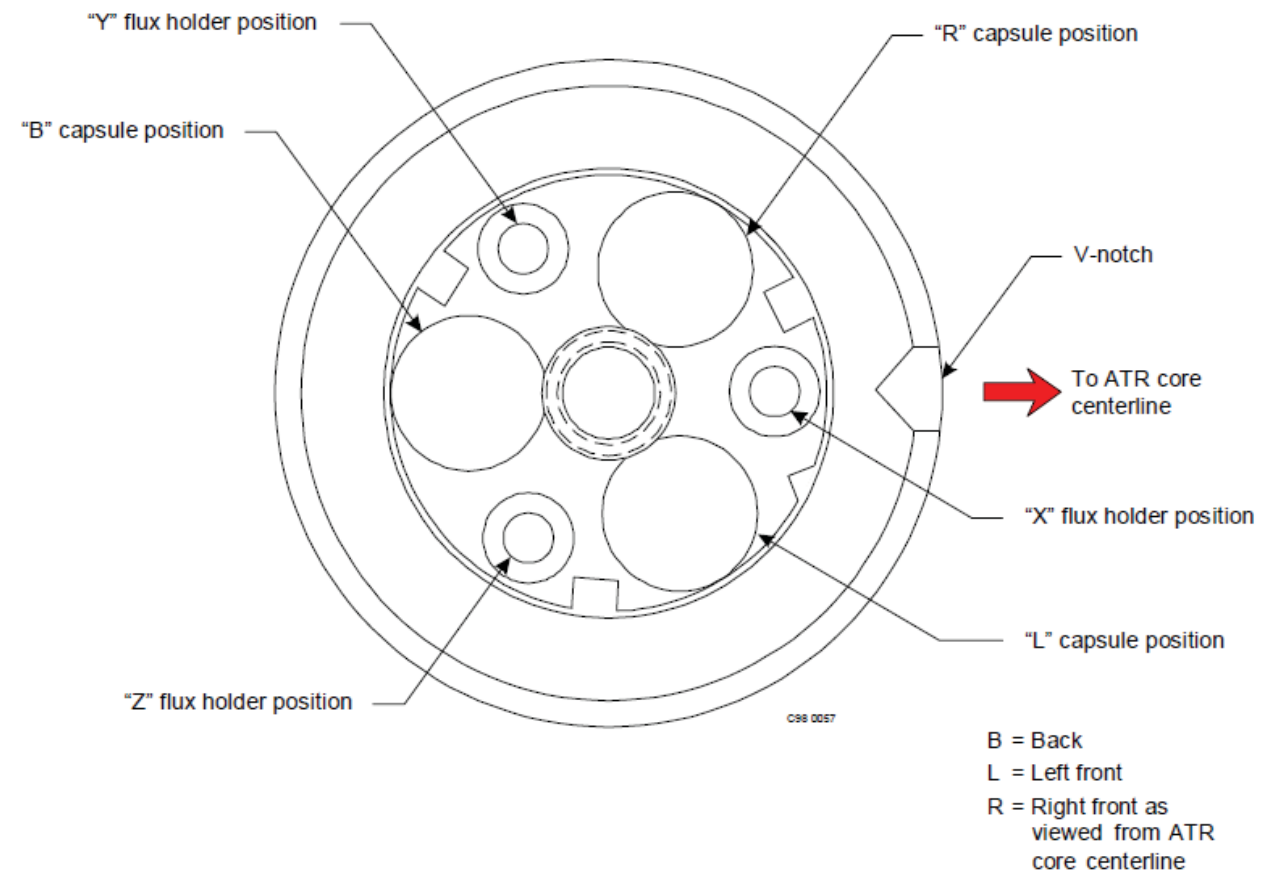

Figure 2. Top view of LWR-2 test assembly for ATR irradiation. 
Examples of ceramic fuel compositions tested under the Advanced Fuel Cycle Initiative (AFCI)/FCR\&D programs are shown in Table 2.

Table 2. Previously-irradiated fast reactor ceramic fuel compositions.

\begin{tabular}{|c|c|}
\hline$\left(\mathrm{U}_{0.80}, \mathrm{Pu}_{0.20}\right) \mathrm{O}_{1.98}$ & $\left(\mathrm{Pu}_{0.5}, \mathrm{Am}_{0.5}\right) \mathrm{N}-36 \mathrm{ZrN}$ \\
\hline$\left(\mathrm{U}_{0.75}, \mathrm{Pu}_{0.20}, \mathrm{Am}_{0.03}, \mathrm{~Np}_{0.02}\right) \mathrm{O}_{1.98}$ & $\left(\mathrm{Pu}_{0.5}, \mathrm{Am}_{0.25}, \mathrm{~Np}_{0.25}\right) \mathrm{N}-36 \mathrm{ZrN}$ \\
\hline$\left(\mathrm{U}_{0.75}, \mathrm{Pu}_{0.20}, \mathrm{Am}_{0.03}, \mathrm{~Np}_{0.02}\right) \mathrm{O}_{1.95}$ & $\left(\mathrm{U}_{0.5}, \mathrm{Pu}_{0.25}, \mathrm{Am}_{0.15}, \mathrm{~Np}_{0.10}\right) \mathrm{N}$ \\
\hline
\end{tabular}

The current reference fast reactor ceramic fuel cladding is HT-9 with 0.230-in.outer diameter (OD) and 0.194-in. inner diameter (ID). Investigations into advanced claddings include new cladding materials [e.g., improved alloys, Oxide Dispersion Strengthened (ODS), etc.] and cladding coatings or liners to prevent interaction between fuel and cladding materials.

Typical fast reactor oxide fuel ATR test conditions are listed in Table 3.

Table 3. Typical fast reactor oxide fuel test conditions in ATR.

\begin{tabular}{|l|c|}
\hline \multicolumn{1}{|c|}{ Parameter } & Typical Value \\
\hline Linear Heat Generation Rate (LHGR) & $350 \mathrm{~W} / \mathrm{cm}$ \\
Peak Cladding Inner Temperature & $550-650{ }^{\circ} \mathrm{C}$ \\
Fuel Centerline Temperature & $2600{ }^{\circ} \mathrm{C}$ \\
Fuel Pin Plenum Pressure & $1250 \mathrm{psi}$ \\
Fast Flux & $\sim 10^{14} \mathrm{n}_{\text {fast }} / \mathrm{cm}^{2}$-s \\
\hline
\end{tabular}

The oxide fuel column is typically 0.191 -in. OD and 1.5-in. long and may consist of multiple pellets. Additional insulator pellets (e.g., $\mathrm{dUO}_{2}$ or $\mathrm{HfO}_{2}$ ) may be placed above and below the fuel column. The fuel pellets should be designed to have a sufficient length-to-diameter ratio to prevent tumbling during fuel loading into the rodlet. The fuel column is held down by a spring to prevent fuel pellet movement during shipping and handling. During fabrication, the miniature fuel rodlet is sealed in a helium environment at a pressure slightly less than atmospheric. For ATR irradiations, the sealed fuel rodlet is encapsulated in an outer capsule (316 SS, 0.354-in. OD, 0.234-in. ID) with a helium backfill at a pressure slightly less than atmospheric. The gas gap between the rodlet OD and the capsule ID provides enough thermal resistance to achieve desired fuel cladding temperatures (hence, desired fuel temperatures). A typical drop-in capsule experiment can include up to 5 capsules (each with one rodlet) stacked vertically in an ATR irradiation position (Outboard A positions). The current vision for an instrumented lead experiment involves the same basic fuel rodlet and capsule design with the same radial dimensions.

\subsubsection{Typical Irradiation Test Design}

The FCR\&D program is currently conducting or designing irradiation experiments for ATR and the HFIR. As discussed in Section 3.1.1, irradiation options in the ATR include drop-in (static) capsules and instrumented lead experiments, and, possibly, the use of hydraulic shuttles (rabbits). The HFIR rabbit system is currently being tested to accommodate Transmission Electron Microscope (TEM) disks and other small fuels and materials specimens.

The basic ATR drop-in capsule design with oxide fuel is shown in Figure 3. 


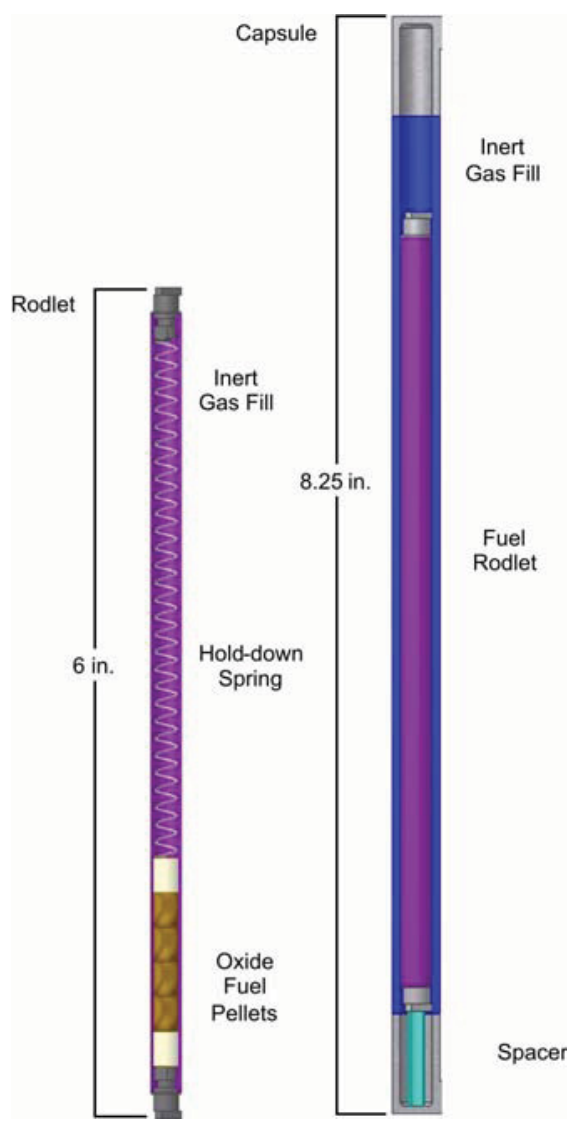

Figure 3. Typical oxide fuel drop-in capsule configuration.

\subsection{Metal Fuel for SFR Operation}

\subsubsection{Description}

Metallic fuels are of primary interest for use in fast reactors. The performance of metallic fuels used to transmute transuranic elements from used nuclear fuel from light water reactors (LWRs) is currently being studied. Typical fast reactor metallic fuel dimensions are based on those used in the EBR-II program. Typical compositions are also based on those used in EBR-II with evolutions to accommodate transuranic species, the potential carryover of lanthanide species during reprocessing, and improved fuel form stability.

Examples of metallic fuel compositions tested under the AFCI/FCR\&D programs are listed in Table 4.

Table 4. Previously-irradiated fast reactor metallic fuel compositions. ${ }^{\text {a }}$

\begin{tabular}{|l|c|c|}
\hline U-10Zr & U-20Pu-3Am-2Np-15Zr & Pu-40Zr \\
U-20Pu-10Zr & U-20Pu-3Am-2Np-1RE-15Zr & Pu-12Am-40Zr \\
U-20Pu-3Am-2Np-10Zr & U-20Pu-3Am-2Np-1.5RE-15Zr & Pu-10Am-10Np-40Zr \\
U-34Pu-4Am-2Np-20Zr & U-30Pu-5Am-3Np-20Zr & U-25Pu-3Am-2Np-40Zr \\
U-28Pu-7Am-30Zr & U-30Pu-5Am-3Np-1RE-20Zr & \\
U-29Pu-4Am-2Np-30Zr & U-30Pu-5Am-3Np-1.5RE-20Zr & \\
\hline
\end{tabular}

a. Metallic compositions expressed in weight percent.

b. $\mathrm{RE}=6 \% \mathrm{La}, 16 \% \mathrm{Pr}, 25 \% \mathrm{Ce}, 53 \% \mathrm{Nd}$ 
The current reference fast reactor metallic fuel cladding is HT-9 with 0.230-in. OD and 0.194-in. ID. Investigations into new claddings include advanced cladding materials (e.g., improved alloys, ODS) and cladding coatings or liners to prevent interaction between fuel and cladding materials.

Typical ATR test conditions for fast rector metallic fuels are listed in Table 5.

Table 5. Typical fast reactor metallic fuel test conditions in ATR.

\begin{tabular}{|l|c|}
\hline \multicolumn{1}{|c|}{ Parameter } & Typical Value \\
\hline Linear Heat Generation Rate (LHGR) & $350 \mathrm{~W} / \mathrm{cm}$ \\
Peak Cladding Inner Temperature & $550-650{ }^{\circ} \mathrm{C}$ \\
Fuel Centerline Temperature & $900-1100{ }^{\circ} \mathrm{C}$ \\
Fuel Pin Plenum Pressure & $1250 \mathrm{psi}$ \\
Fast Flux $(>1 \mathrm{MeV})$ & $\sim 3-5 \times 10^{14} \mathrm{n}_{\mathrm{fast}} / \mathrm{cm}^{2}-\mathrm{s}$ \\
\hline
\end{tabular}

\subsubsection{Typical Irradiation Test Design}

The FCR\&D program is currently conducting or designing irradiation experiments for the ATR and the HFIR. Irradiation options in the ATR include the drop-in (static) capsules and instrumented lead experiments and, possibly, the use of the hydraulic shuttles (rabbits) discussed in Section 3. The HFIR rabbit system is currently being tested to accommodate TEM disks and other small fuels and materials specimens.

The basic ATR drop-in capsule design is shown in Figure 4.

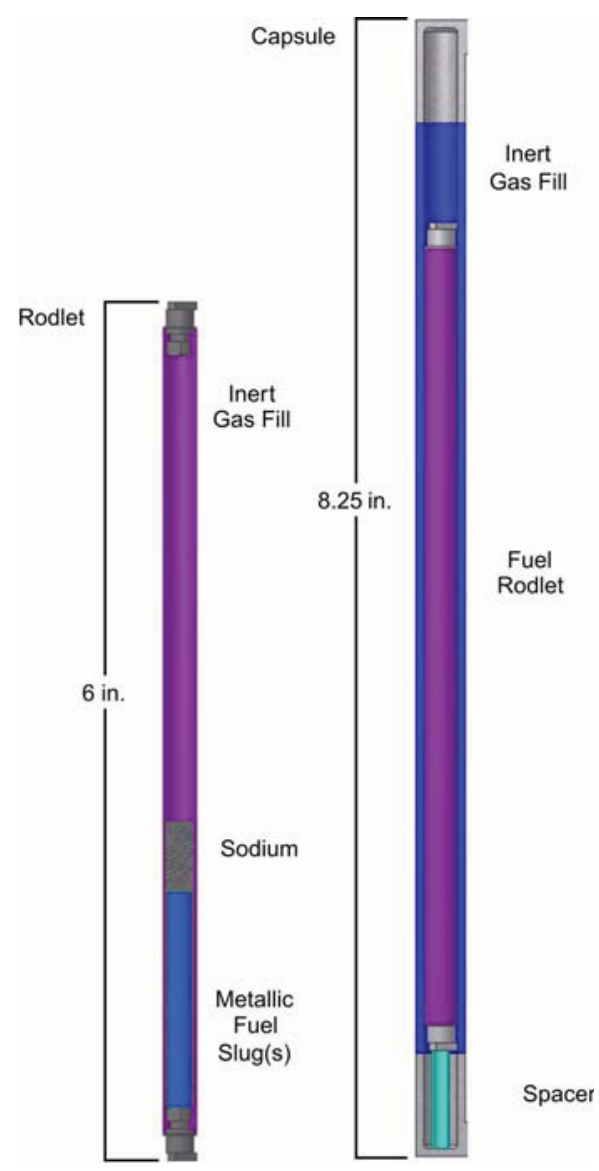

Figure 4. Typical metallic fuel drop-in capsule configuration. 
The metallic fuel column is typically 0.168 -in OD and 1.5 -in. long and may consist of multiple pieces. The fuel column is surrounded by molten sodium which forms a bond between the fuel OD and the cladding ID, ensuring good thermal conductivity (and low fuel centerline temperatures). Initially, the molten sodium covers the top of the fuel by 0.25 to $0.75 \mathrm{in}$. As the fuel swells and expands during irradiation, the molten sodium expands in these gaps, increasing the overall height of the sodium in the capsule. During fabrication, the miniature fuel rodlet is sealed in a helium environment at a pressure slightly less than atmospheric. For ATR irradiations, the sealed fuel rodlet is encapsulated in an outer capsule (316 SS, 0.354-in. OD, 0.234-in. ID) with a helium backfill at a pressure slightly less than atmospheric. The gas gap between the rodlet OD and the capsule ID provides enough thermal resistance to achieve desired fuel cladding temperatures (hence, desired fuel temperatures). A typical drop-in capsule experiment can include up to 5 capsules (each with one rodlet) stacked vertically in an ATR irradiation position (the Outboard A positions discussed Section 3). The current vision for an instrumented lead experiment involves the same basic fuel rodlet and capsule design with the same radial dimensions.

\subsection{Desired Phenomena to Detect}

Irradiation experiments are designed to determine behavior within the fuel and between fuel and cladding materials. During irradiation, the fuel and cladding are subjected to temperature and flux gradients, and fission products and transmutation products are generated from interactions with neutrons.

Microstructural changes may occur due to temperature gradients, as some fuel constituents may migrate in the presence of a temperature gradient. Microstructural changes also occur as the fuel composition changes and the fuel matrix accommodates fission products (solid and gaseous) and changing stoichiometry (especially in ceramic fuels).

Constituent redistribution is an important process because changes in concentration within the fuel pin can lead to changes in phase and to low-melting temperature eutectic formation. Examples of constituent redistribution in metallic fast reactor fuels, along with their corresponding element maps, are shown in Figure 5 .

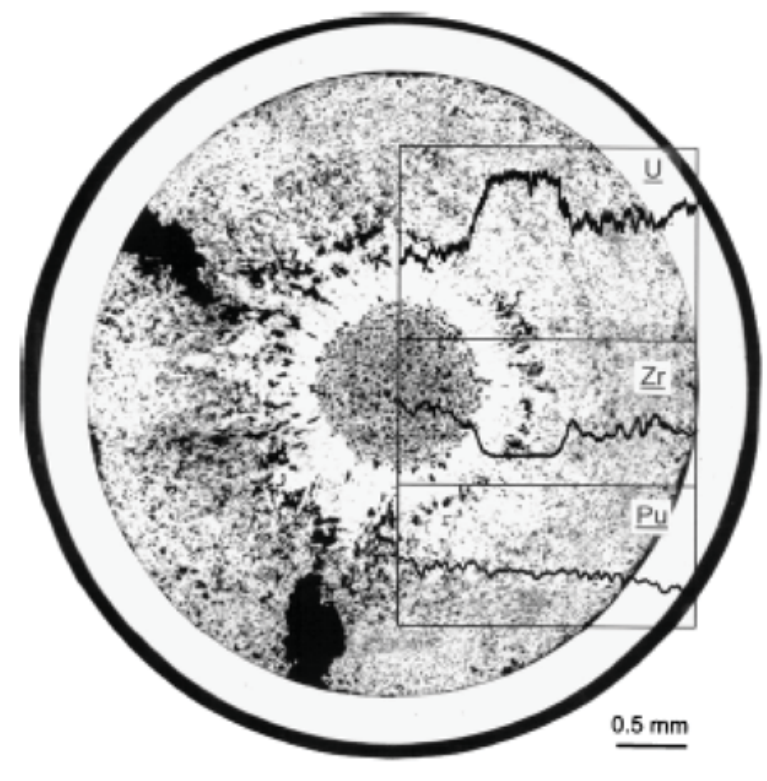

$\mathrm{U}-\mathrm{Pu}-\mathrm{Zr}$

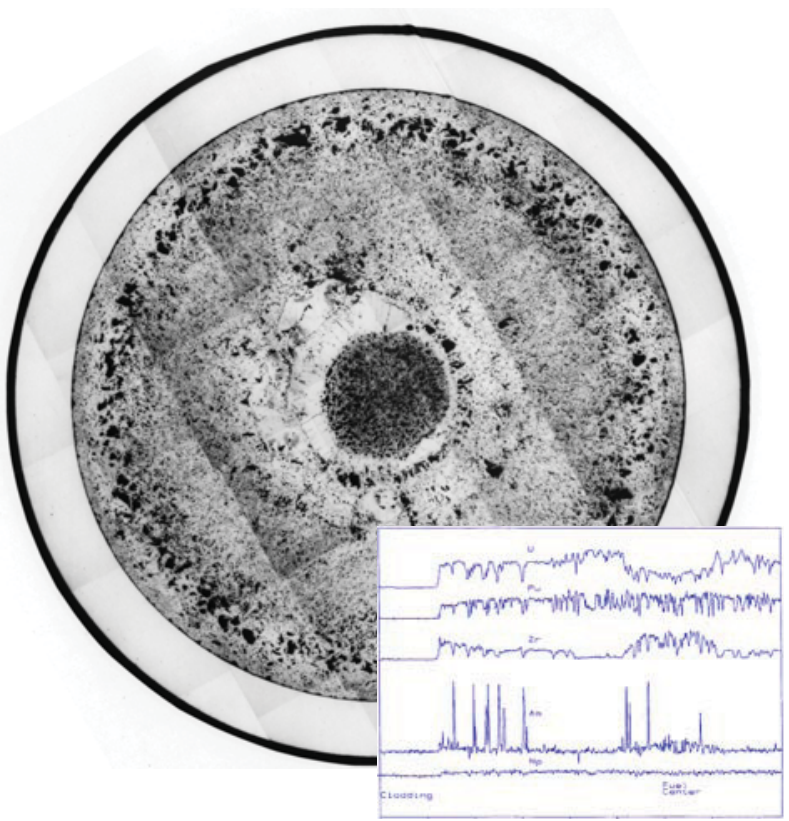

U-Pu-Am-Np-Zr

Figure 5. Examples of constituent redistribution in metallic fast reactor fuels. 
Fission product creation (including solid and gaseous fission products) leads to fuel swelling. The amount of swelling for any particular fuel form and composition is important to know because the initial fuel and cladding sizes must be designed to accommodate swelling without producing unacceptable mechanical interaction between fuel and cladding. Figure 6 shows examples of metallic fuel swelling and restructuring and indicates that swelling and restructuring occur early in irradiation. Short-term irradiations of MOX fuel and minor actinide-containing MOX fuel conducted by Japan Atomic Energy Agency (JAEA) show that restructuring and redistribution begins within the first 24 hours of irradiation and can even be seen as early as 10 minutes. ${ }^{12}$

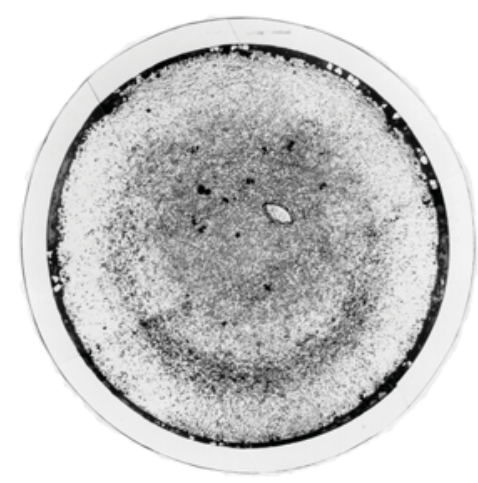

X423 at $0.9 \%$ burnup

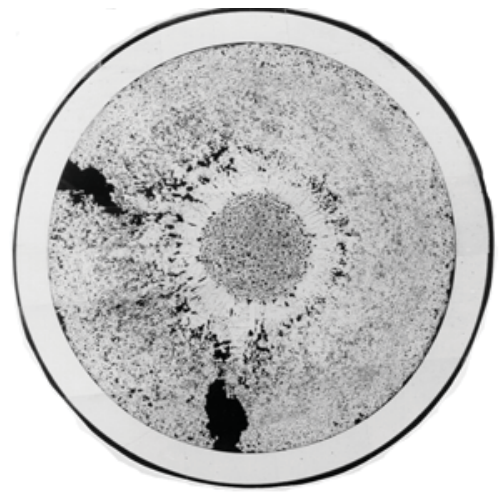

X419 at 3\% burnup

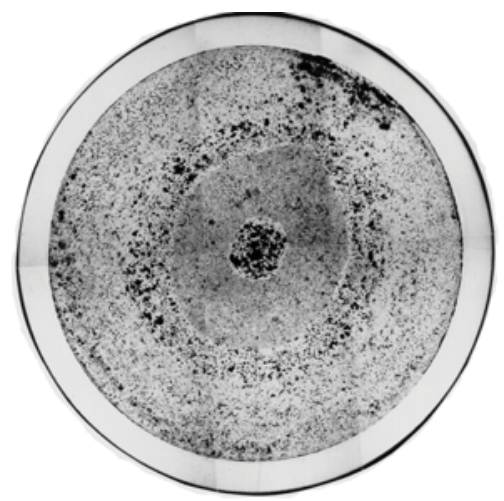

X420B at $17 \%$ burnup

Figure 6. Swelling and restructuring of U-20Pu-10Zr fuel.

Initially, fuel may be either fully dense or fabricated with some initial porosity. During irradiation, fuel porosity increases due to restructuring, irradiation damage, and the accumulation of fission products, and at some point, the porosity becomes interconnected and gases produced during fission can escape from the fuel matrix, leading to fission gas release. This released gas increases pressure in the fuel rod plenum. It is important to track fission gas release to determine how fuel composition (especially the transuranics) effects total fission gas release and release rates. An example of fission gas release for a few metallic fuel compositions is shown in Figure 7.

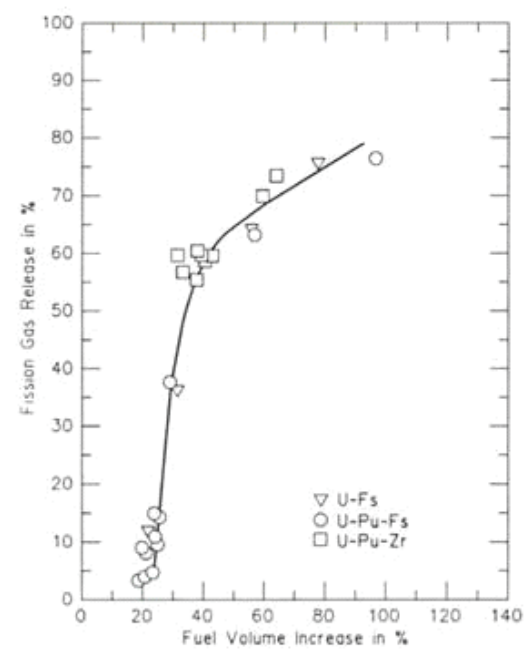

Figure 7. Fission gas release for fast reactor metallic fuel compositions. 
Thermal and mechanical forces and changing composition may lead to cracking and other microstructural changes during irradiation. Physical changes in the fuel may change thermal transport and diffusion pathways, leading to increased fission product or fission gas release or thermal anomalies.

In addition to fuel swelling, the cladding may experience creep during irradiation. Eventually, the fuel and cladding usually come into contact. Fuel constituents, especially some fission products or reprocessing carryover, may interact with the cladding, reducing the effective strength of the cladding. Understanding and predicting fuel-cladding interaction is important to determine ultimate fuel rod lifetimes. Examples of fuel-cladding interaction with catastrophic failure and general fuel restructuring are shown in Figure 8.

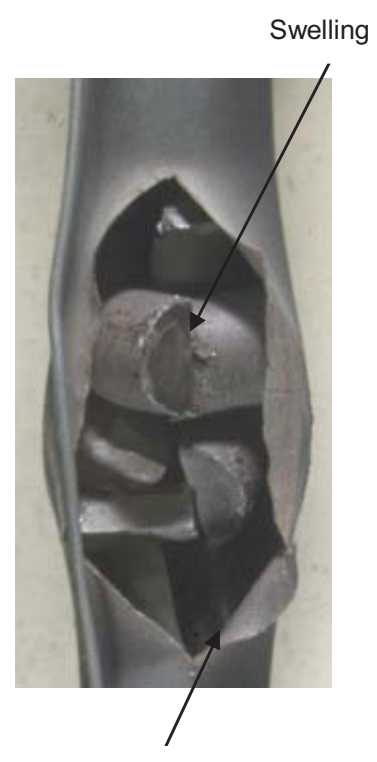

P-C interaction

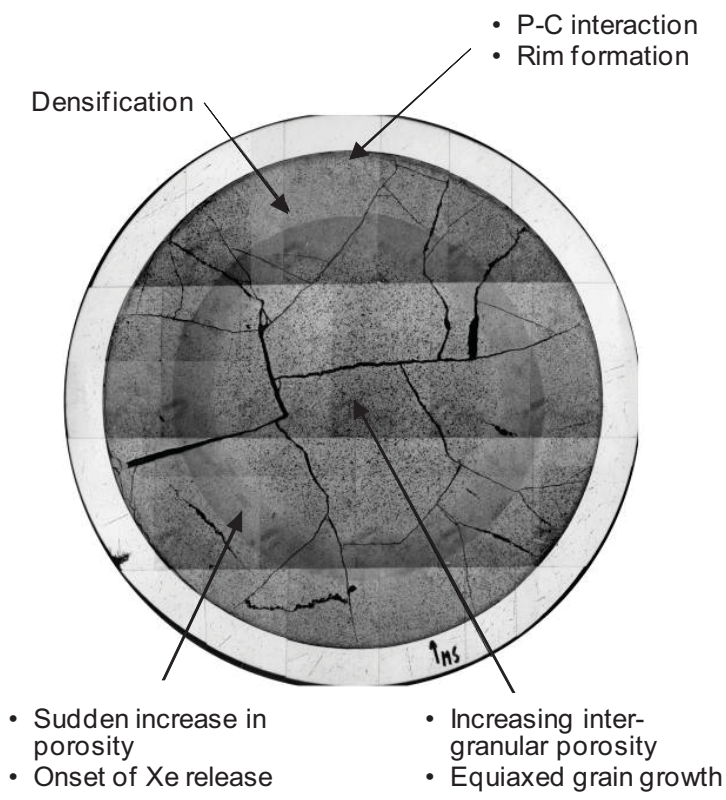

Figure 8. Examples of catastrophic fuel failure (left) and fuel restructuring (right).

Important metallic fuel performance phenomena include:

- irradiation growth;

- fuel swelling and fuel-cladding mechanical interaction (FCMI);

- gas release;

- fuel constituent redistribution;

- fuel-cladding chemical interaction (FCCI).

Important oxide fuel performance phenomena include:

- fuel swelling and fuel-cladding mechanical interaction (FCMI);

- fuel restructuring;

- gas release;

- fuel-cladding chemical interaction (FCCI);

- fuel-coolant compatibility. 


\subsection{Desired Physical Parameters to Detect}

In-situ instrumentation is desired to provide real-time data on fuel performance phenomena. Without wireless transmission capabilities, drop-in or static capsule experiments only allow data to be obtained at the endpoint of an experiment. In-situ instrumentation in instrumented tests can provide data that shows the evolution of particular phenomena over time. Selected parameters of interest for fuel modeling, with currently-requested accuracies, ${ }^{13,14}$ are listed in Table 6.

Table 6. Summary of desired parameters for detection during fuel irradiation tests.

\begin{tabular}{|c|c|c|c|}
\hline \multirow{2}{*}{ Parameter } & \multirow{2}{*}{ Representative - Peak Value } & \multicolumn{2}{|c|}{ Desired } \\
\hline & & Accuracy & Spatial Resolution \\
\hline \multirow[t]{3}{*}{\begin{tabular}{|l} 
fuel temperature \\
\end{tabular}} & Ceramic LWR $-1400{ }^{\circ} \mathrm{C}$ & \multirow[t]{3}{*}{$2 \%$} & \multirow{3}{*}{$\begin{array}{l}1-2 \mathrm{~cm} \text { (axially); } \\
0.5 \mathrm{~cm} \text { (radially) }\end{array}$} \\
\hline & Ceramic SFR- $2600^{\circ} \mathrm{C}$ & & \\
\hline & Metallic SFR $-1100^{\circ} \mathrm{C}$ & & \\
\hline \multirow[t]{3}{*}{\begin{tabular}{|l} 
cladding temperature \\
\end{tabular}} & Ceramic LWR $-<400{ }^{\circ} \mathrm{C}$ & \multirow[t]{3}{*}{$2 \%$} & \multirow[t]{3}{*}{$1-2 \mathrm{~cm}$ (axially) } \\
\hline & Ceramic SFR $-650^{\circ} \mathrm{C}$ & & \\
\hline & Metallic SFR $-650^{\circ} \mathrm{C}$ & & \\
\hline \multirow[t]{3}{*}{ pressure in fuel rod plenum } & Ceramic LWR - 800 psi & \multirow[t]{3}{*}{$5 \%$} & \multirow[t]{3}{*}{$\mathrm{NA}^{\mathrm{a}}$} \\
\hline & Ceramic SFR-1250 psi & & \\
\hline & Metallic SFR - $1250 \mathrm{psi}$ & & \\
\hline $\begin{array}{l}\text { fission gas release (amount } \\
\text { and composition) }\end{array}$ & $0-100 \%$ of inventory & $10 \%$ & NA \\
\hline \multirow{3}{*}{$\begin{array}{l}\text { fuel and cladding } \\
\text { dimensions radial and axial } \\
\text { (includes fuel-cladding gap } \\
\text { size) }\end{array}$} & Initial Length, $1 \mathrm{~cm}$ & $1 \%$ & NA \\
\hline & Outer diameter/Strain, $0.5 \mathrm{~cm} / 5-10 \%$ & $0.1 \%$ & NA \\
\hline & Fuel-Cladding Gap (0-0.1 mm) & $0.1 \%$ & NA \\
\hline \multirow{3}{*}{$\begin{array}{l}\text { fuel morphology/ } \\
\text { microstructure/cracking/ } \\
\text { constituent redistribution }\end{array}$} & Grain size, $10 \mu \mathrm{m}$ & $5 \%$ & $1-10 \mu \mathrm{m}$ \\
\hline & Swelling/Porosity, 5-20\% & $2 \%$ & \\
\hline & Crack formation and growth & $2 \%$ & $10-100 \mu \mathrm{m}$ \\
\hline \multirow[t]{2}{*}{ fuel thermal properties } & $\begin{array}{c}\text { Thermal conductivity, } \\
\text { Ceramic: }<5 \mathrm{~W} / \mathrm{mK} \text {; Metallic: }<50 \mathrm{~W} / \mathrm{mK}\end{array}$ & $4 \%$ & $<1 \mathrm{~cm}$ (radially) \\
\hline & $\begin{array}{l}\text { Density (estimated from changes in length, } \\
\text { diameter, porosity, etc.) }\end{array}$ & $2 \%$ & NA \\
\hline \multirow{2}{*}{$\begin{array}{l}\text { neutron flux for estimating } \\
\text { fluence and fuel burnup }\end{array}$} & Thermal neutron flux - $1-5 \times 10^{14} \mathrm{n} / \mathrm{cm}^{2}-\mathrm{s}$ & $1-10 \%$ & $5 \mathrm{~cm}$ (axially) \\
\hline & $\begin{array}{l}\text { Fast neutron flux }(\mathrm{E}>1 \mathrm{MeV})-\sim 1-5 \times 10^{14} \\
\qquad \mathrm{n} / \mathrm{cm}^{2}-\mathrm{s}\end{array}$ & $15 \%$ & $5 \mathrm{~cm}$ (axially) \\
\hline
\end{tabular}

a. NA-Not Applicable.

Although all of the above parameters are of interest for modeling fuel behavior, the FCR\&D program has placed the highest priority on sensors that can provide insights related to fuel morphology because such insights are needed to clarify changes in other parameters, such as thermal conductivity, density, etc.

As discussed previous, it is desirable to obtain data from prototype fuel operating conditions. However, to obtain the desired parameters with the desired accuracies, non-prototypic test conditions may be necessary that will allow higher precision in-pile instrumentation to be deployed. This subject is addressed further in Section 6. 


\section{IRRADIATION OPTIONS}

As discussed previously, FCR\&D irradiations are primarily performed in INL's ATR and ORNL's HFIR. Because of their high flux levels, these facilities offer unique opportunities for evaluating fuel performance under irradiation. Although this section is primarily devoted to describing irradiation options in these facilities, this section also discusses other representative irradiation options available or that could become available to the FCR\&D effort. Although instrumentation development efforts are primarily focussed on ATR and HFIR test conditions, requirements associated with other possible irradiation facilities will be identified in this program.

\subsection{Material and Test Reactors}

As noted above, most FCR\&D irradiations are performed in the ATR and HFIR Key design parameters and characteristics for each of these facilities are highlighted in this section. Irradiation locations in these two facilities are also discussed and compared with other material test reactors (MTRs).

\subsubsection{ATR}

The ATR is a unique facility for scientific investigation of nuclear fuel and materials. ${ }^{15}$ Designed to allow simulation of long neutron radiation exposures in a short time period, the ATR has a maximum power rating of $250 \mathrm{MW}_{\text {th }}$ with a maximum unperturbed thermal neutron flux of $1 \times 10^{15} \mathrm{n} / \mathrm{cm}^{2}$-s and a maximum fast neutron flux of $5 \times 10^{14} \mathrm{n} / \mathrm{cm}^{2}$-s.

The ATR is cooled by pressurized $(2.5 \mathrm{MPa} / 360 \mathrm{psig})$ water that enters the reactor vessel bottom at an average temperature of $52^{\circ} \mathrm{C}\left(126^{\circ} \mathrm{F}\right)$, flows up outside cylindrical tanks that support and contain the core, passes through concentric thermal shields into the open part of the vessel, then flows down through the core to a flow distribution tank below the core. When the reactor is operating at full power, the primary coolant exits the vessel at $71^{\circ} \mathrm{C}\left(160^{\circ} \mathrm{F}\right)$.

\subsubsection{Reactor Design and Characteristics}

As shown in Figure 9, the ATR core consists of 40 curved plate fuel elements in a serpentine arrangement around a 3 x 3 array of primary testing locations, or nine large high-intensity neutron flux traps. The unique ATR control device design permits large power variations among its nine flux traps using a combination of control cylinders (drums) and neck shim rods. The beryllium control cylinders contain hafnium plates that can be rotated toward and away from the core. Hafnium shim rods, which withdraw vertically, are inserted or withdrawn for minor power adjustments. Within bounds, the power level in each corner lobe of the reactor can be controlled independently to allow for different power and flux levels in the four corner lobes during the same operating cycle. The ratio of fast to thermal flux can be varied from 0.1 to 1.0. In addition to the nine large volume (up to 48" long and up to 5.0" diameter) high-intensity neutron flux traps, there are 66 irradiation positions inside the reactor core reflector tank, and there are two capsule irradiation tanks outside the core with 34 low-flux irradiation positions. A Hydraulic Shuttle Irradiation System (HSIS), more commonly referred to as the "Rabbit", was also recently installed in the ATR to restore the reactor's capability to perform irradiations of small capsules in the B-7 position of the reactor for materials research, rapid activations, and isotope production. ${ }^{16}$

\subsubsection{Test Configurations and Conditions}

Irradiated samples are enclosed in test capsules that are then typically placed in a basket to facilitate positioning within the reactor. This section provides summary information about the three ATR primary test configurations, which are conceptually shown in Figure 10, and the HSIS. More detailed information can be found in References 15 and 16. 


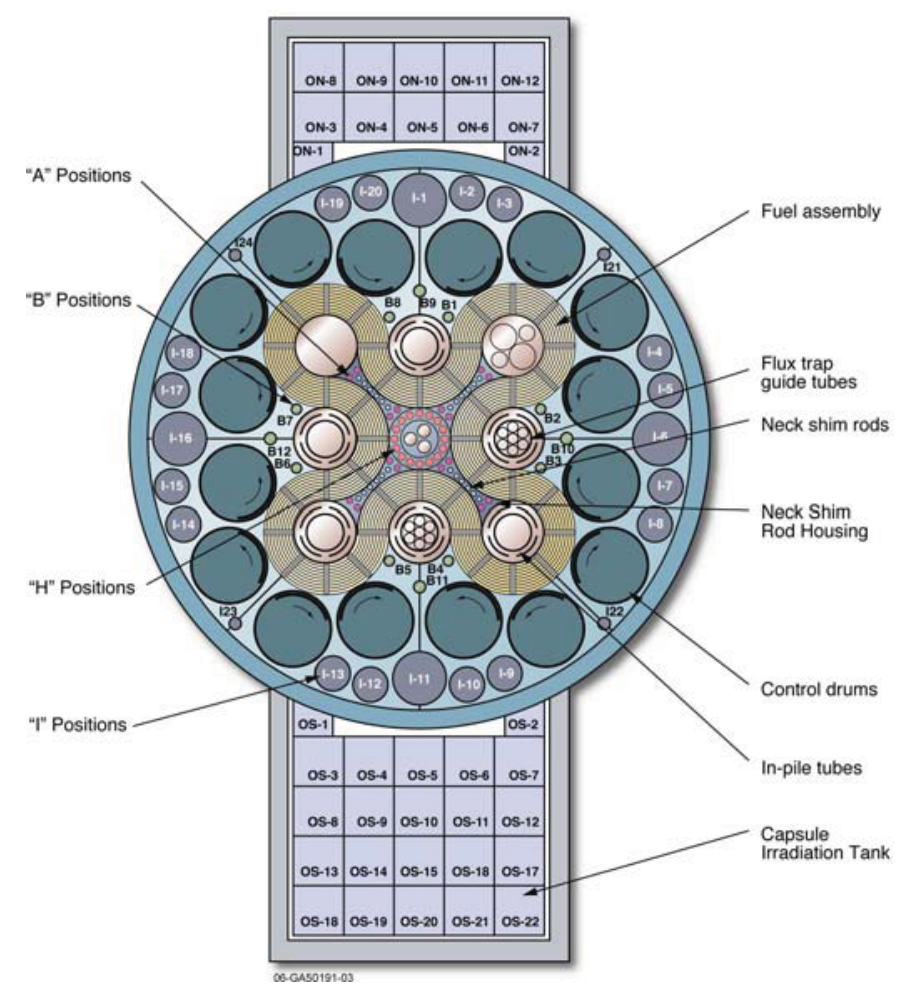

Figure 9. ATR core cross section showing irradiation locations.
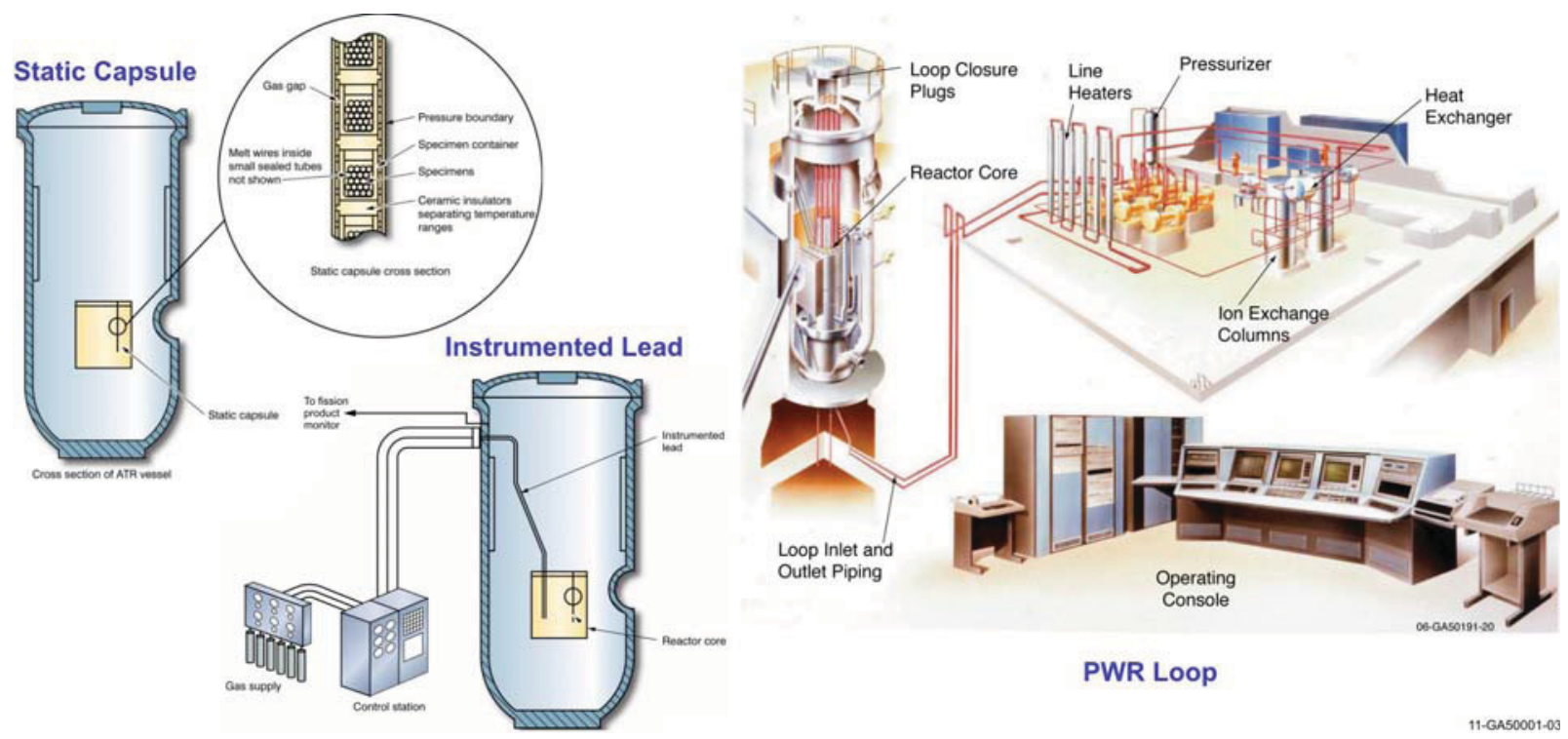

PWR Loop

Figure 10. Schematic diagrams illustrating ATR irradiation locations.

- Static Capsule Experiments - These capsules may contain a number of small samples or engineered components. Static capsule experiments may be sealed or may contain material that can be in contact with the ATR primary coolant (such capsules are in an open configuration without being sealed). Capsules may be any length, up to $122 \mathrm{~cm}$ (48 in.) and may be irradiated in any core position, including the flux traps. Irradiation temperature may be selected by providing a gas gap in the capsule with a known thermal conductance. Peak temperatures may be measured using a 
series of melt wires, temperature-sensitive paint spots, or silicon carbide temperature monitors. Accumulated neutron fluences may be verified using flux wires.

- Instrumented Lead Experiments - Active control of experiments and data from test capsules during irradiation is achieved using core positions with instrumentation cables and temperature control gases in ATR instrumented lead experiments. Such experiments can have instrumentation, such as thermocouples, connected to individual capsules or single specimens. This instrumentation can be used to control and sample conditions within the capsule. For example, temperature control in individual zones is performed by varying the gas mixture (typically helium and neon) in the gas gap that thermally links the capsule to the water-cooled reactor structure. In addition to temperature, instrumented lead experiments can be configured to monitor the gas around the test specimen. In a fueled experiment, the presence of fission gases due to fuel failures or oxidation can be detected via gas chromatography. Instrument leads allow real time display of experimental parameters in the control room.

- Pressurized Water Loop Experiments - Five of the nine ATR flux traps used for materials and fuels testing are equipped with pressurized water loops (at the NW, N, SE, SW, and W locations). A sixth loop will be operational in 2011. Each of the water loops can be operated at different temperatures, pressures, flow rates, or water chemistry requirements. These loops can operate above the standard temperatures and pressure of a commercial PWR power plant. The great advantage of loop tests is the ease with which a variety of samples can be subjected to conditions specified for any PWR design. Each ATR pressurized loop is instrumented to measure and control coolant flows (both helium and water), temperatures, pressures and sample test data.

- Rabbit Tests - The HSIS or rabbit enables insertion and removal of experiment specimens during ATR during operational cycles. The HSIS is installed in the B-7 reflector position, which is one of the higher flux positions in the reactor with typical thermal and fast $(>1 \mathrm{MeV})$ fluxes of $2.8 \mathrm{E}+14 \mathrm{n} /$

$\mathrm{cm}^{2} / \mathrm{sec}$ and $1.9 \mathrm{E}+14 \mathrm{n} / \mathrm{cm}^{2} / \mathrm{sec}$, respectively. The titanium experiment capsules, or shuttles, are approximately $16 \mathrm{~mm}$ in diameter $\mathrm{x} 57 \mathrm{~mm}$ in length with interior usable dimensions of $14 \mathrm{~mm}$ in diameter x $50 \mathrm{~mm}$ long. Up to 14 capsules can be used for irradiations simultaneously, although one does not need to fill all 14 capsules for a test. The maximum allowable weight of each shuttle contents is 27.0 grams.

\subsubsection{HFIR}

The HFIR is a beryllium-reflected, pressurized, light-water-cooled and moderated flux-trap-type reactor. The core, which consists of aluminum-clad involute-fuel plates, currently utilizes highly enriched ${ }^{235} \mathrm{U}$ fuel, with a design power level of 85 MW. The HFIR was originally designed (in the 1960s) to primarily support the overall program to produce transuranic isotopes for use in the U.S. heavy-element research program. Today, the reactor is a highly versatile machine, producing medical and transuranic isotopes, and performing materials test experimental irradiations and neutron-scattering experiments, including the capability to conduct cold-source low-temperature neutron experiments.

\subsubsection{Reactor Design and Characteristics}

The reactor core, illustrated in Figure 11, consists of a series of concentric annular regions, each approximately $61 \mathrm{~cm}$ in height. The flux trap is $\sim 12.7 \mathrm{~cm}$ in diameter, and the outer fueled region is $\sim 43.5 \mathrm{~cm}$ in diameter. The fuel region is surrounded by a concentric ring of beryllium reflector approximately $30.5 \mathrm{~cm}$ in thickness. The beryllium reflector is in turn backed up by a water reflector of effectively infinite thickness. In the axial direction, the reactor is reflected by water. The reactor core assembly is contained in a $2.44 \mathrm{~m}$ diameter pressure vessel, which is located in a $5.5 \mathrm{~m}$ cylindrical pool of water. Figure 12 shows a 
cutaway of the reactor pressure vessel, its location in the reactor pool. Experiment facilities shown in this figure are discussed in Section 3.1.2.2.

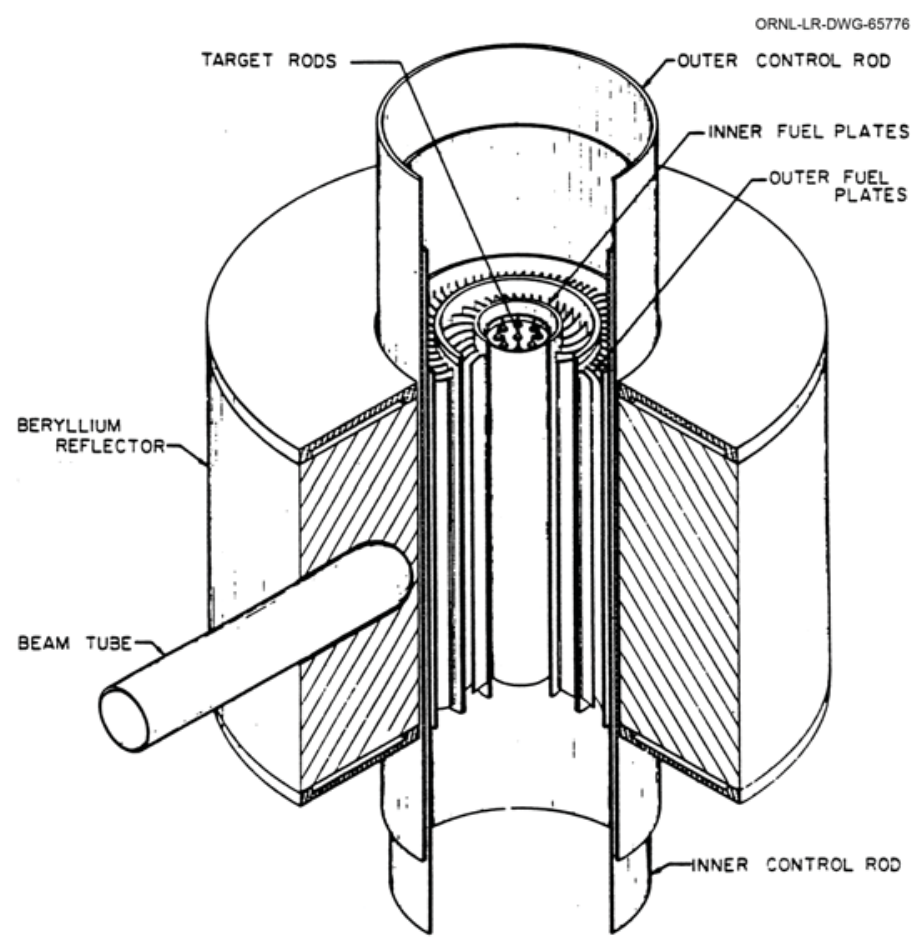

Figure 11. Schematic of HFIR reactor core and beryllium reflector.

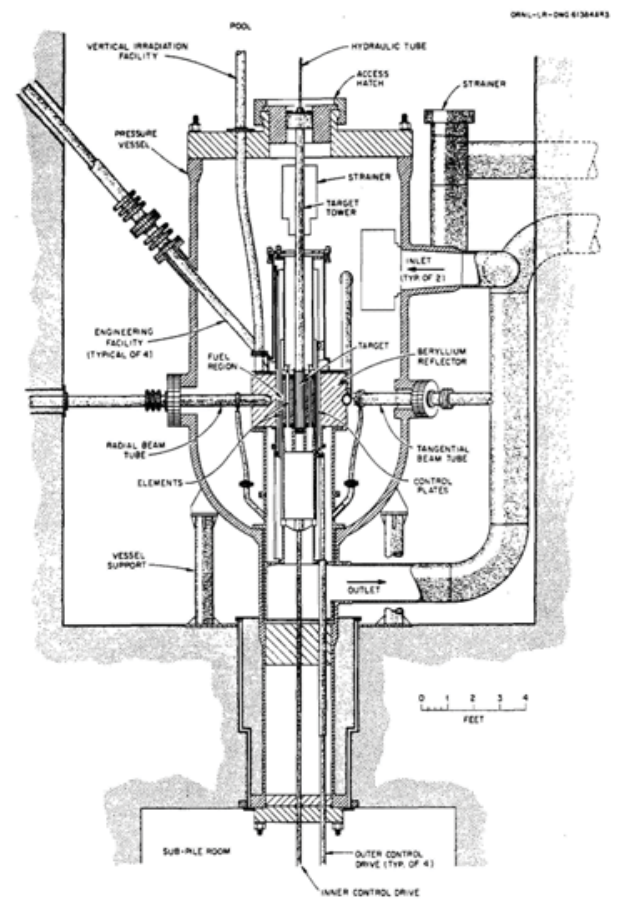

Figure 12. Cross section through HFIR pressure vessel. 


\subsubsection{Test Configurations and Conditions}

Several facilities within the HFIR core and reflector are available for experimental use. These include (1) the flux trap, (2) three horizontal beam holes which originate in the reflector, (3) four slant access facilities which are located adjacent to the outer reflector at an angle with the vertical, and (4) 30 vertical facilities of various sizes located in the reflector. Figure 13, a cross section of the HFIR, illustrates these experimental facilities.

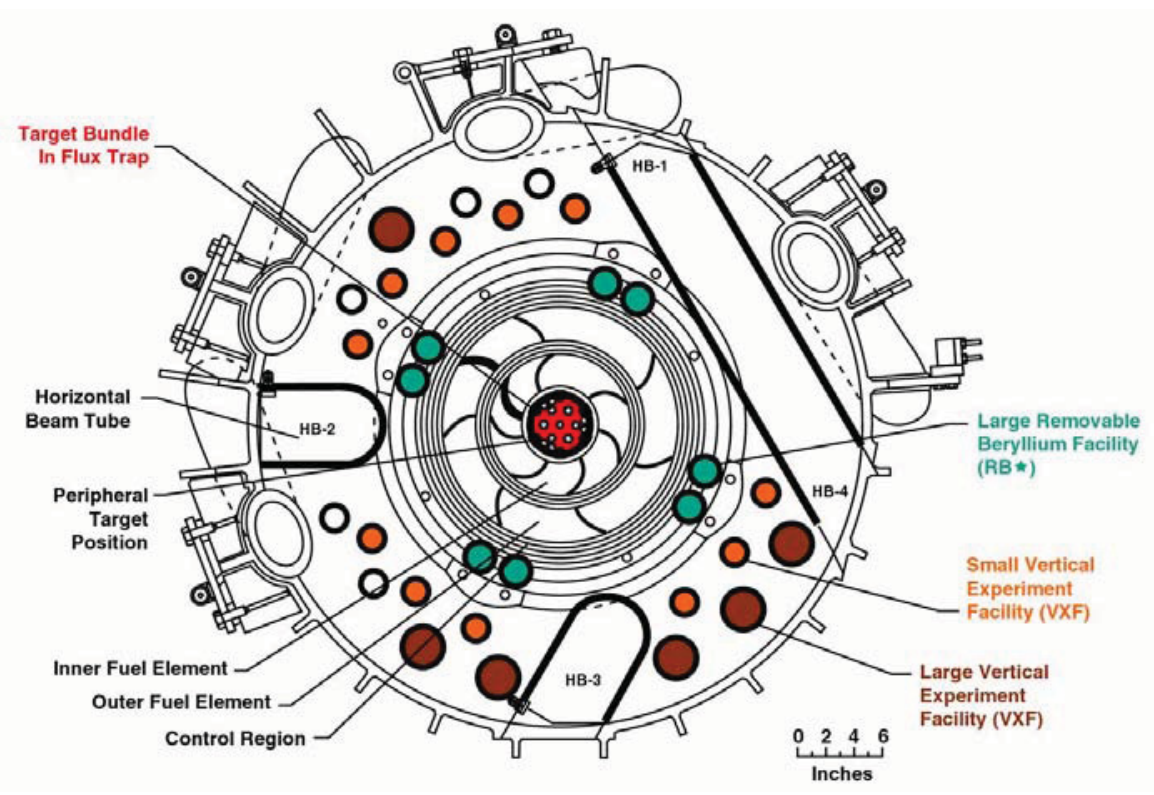

Figure 13. Cross section through HFIR mid-plane.

Table 7 contains the characteristics of the vertical irradiation facilities in HFIR. The facilities listed start with the flux trap (left column) and proceed outward to the large Vertical Experiment Facility (VXF) positions (right-most column). Going from the target region to the large VXF position, the fast flux decreases by a factor of 100 and the thermal flux (without shields) decreases by a factor of 5. Also given in Table 7 are the characteristics for the Large Removable Beryllium Facility (RB*) position with and without thermal-neutron shields. Using the standard $\mathrm{RB}^{*} \mathrm{Eu}_{2} \mathrm{O}_{3}$ shield, the thermal neutron flux can be reduced by a factor of 50 without significantly affecting the fast flux, even after 8-10 cycles of operation. At beginning of life, the fast/thermal ratio is far higher. Thus, it is possible to tailor the neutron spectrum for specific experimental purposes and goals.

Table 7. Characteristics of vertical HFIR irradiation facilities.

\begin{tabular}{lccccc}
\hline \multicolumn{1}{c}{ Characteristic } & Target & $\begin{array}{c}\text { RB* } \\
\text { unshielded }\end{array}$ & $\begin{array}{c}\mathbf{R B}^{*} \text { with } \\
\mathbf{E u}_{\mathbf{2}} \mathbf{O}_{\mathbf{3}} \text { shield }\end{array}$ & $\begin{array}{c}\text { Small } \\
\text { VXF }\end{array}$ & $\begin{array}{c}\text { Large } \\
\text { VXF }\end{array}$ \\
\hline Fast flux, $\mathrm{E}>0.1 \mathrm{MeV}\left(10^{14} \mathrm{n} / \mathrm{cm}^{2} \cdot \mathrm{sec}\right)$ & 11 & 5.3 & 4.9 & 0.5 & 0.13 \\
Thermal flux, $\mathrm{E}<0.1 \mathrm{MeV}\left(10^{14} \mathrm{n} / \mathrm{cm}^{2} \cdot \mathrm{sec}\right)$ & 21 & 11 & 0.19 & 7.5 & 4.3 \\
Peak displacements per atom $(\mathrm{dpa})$ per cycle, stainless steel & 1.8 & 0.67 & 0.58 & & \\
Typical capsule diameter $(\mathrm{mm})$ & 16 & 43 & 38 & 37 & 69 \\
Number of available positions & 36 & 8 & 2 & 16 & 6 \\
\hline
\end{tabular}


The target region has the highest reactor neutron flux (thermal flux $[\mathrm{E}<0.5 \mathrm{eV}]$ of $2.1 \cdot 10^{15} \mathrm{n} / \mathrm{cm}^{2} \cdot \mathrm{sec}$ and a fast flux $[\mathrm{E}>0.1 \mathrm{MeV}]$ of $1.1 \cdot 10^{15} \mathrm{n} / \mathrm{cm}^{2} \cdot \mathrm{sec}$ ). A schematic of the target region is given in Figure 14 . The flux trap contains 36 positions plus the hydraulic tube (rabbit irradiation). Target positions may be irradiated for a cycle or multiple cycles (a cycle averages $\sim 23$ days but may vary from 21-26 days, depending on the power level and experiments loaded in the core). The target region may accommodate two fully instrumented experiments (the E3 and E6 locations).

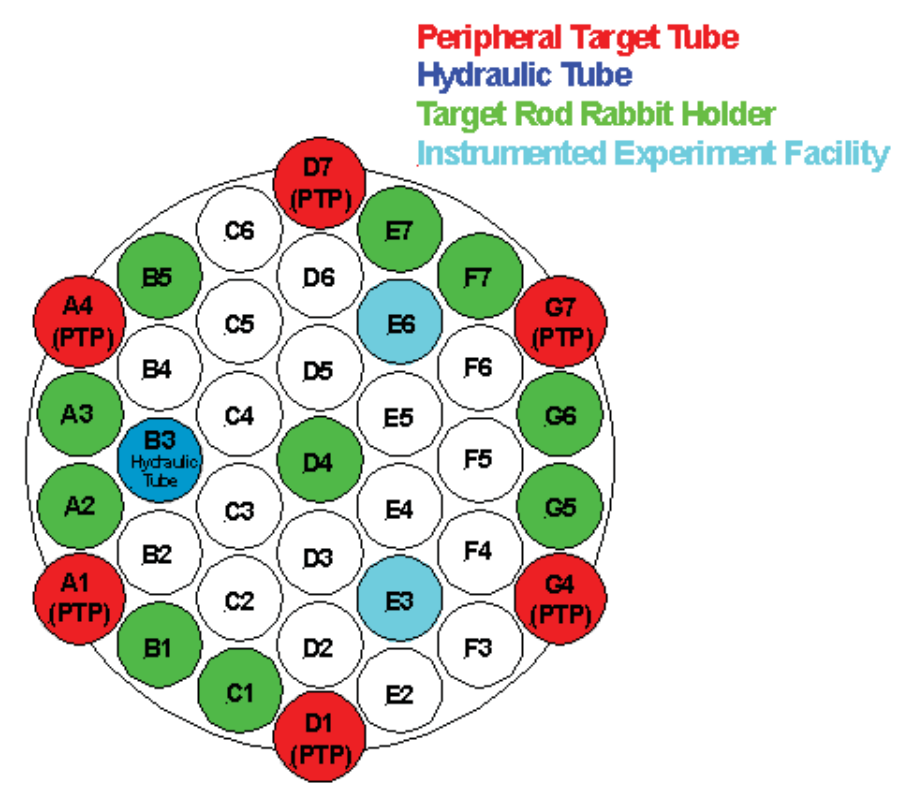

Figure 14. HFIR flux trap schematic.

The target region also contains a hydraulic facility (location B3) which offers the unique opportunity to irradiate experiments for very low doses with irradiation times as short as 1 minute or as long as the full cycle.

The primary vertical test positions in the reflector (outside the core) are the "RB", "small VXF", and the "large VXF" positions (see Figure 13). The attributes (number of available positions, typical experimental capsule diameters, gamma heating and flux levels) for these positions are given in Table 7. Shielded designs are routinely used for these positions with highly instrumented assemblies normally being inserted in the RB positions.

\subsubsection{ATRC}

Located at the INL, the ATR Critical (ATRC) facility is a full-size nuclear mock-up of the ATR core that allows researchers to characterize in advance, with precision and accuracy, the expected changes in ATR core reactivity due to a proposed test. This facility generally operates at a thermal power of less than $5 \mathrm{~kW}$ (with associated peak thermal fluxes of around $10^{10} \mathrm{n} / \mathrm{cm}^{2}-\mathrm{s}$ and a maximum fast neutron flux of around $\left.10^{9} \mathrm{n} / \mathrm{cm}^{2}-\mathrm{s}\right)$.

\subsubsection{Reactor Design and Characteristics}

The primary difference between the ATRC and ATR is that the highly-enriched uranium fuel in the ATRC is uniformly loaded with boron while the ATR fuel is not. A second difference of note is that the ATRC uses five cadmium-plated safety rods while the ATR uses six hafnium-plated safety rods. During operation, this difference is insignificant. ATRC criticality is normally attained at a power greater than 
$0.25 \mathrm{~mW}$. However, this pool-type reactor (Figure 15) usually operates at a power level of about $600 \mathrm{~W}$ and provides useful physics data for evaluating:

- worth and calibration of control elements,

- excess reactivities and charge lifetimes,

- thermal and fast neutron distributions,

- gamma heat generation rates,

- fuel loading requirements,

- effects of inserting and removing experiments and experiment void reactivities, and temperature and void reactivity coefficients.

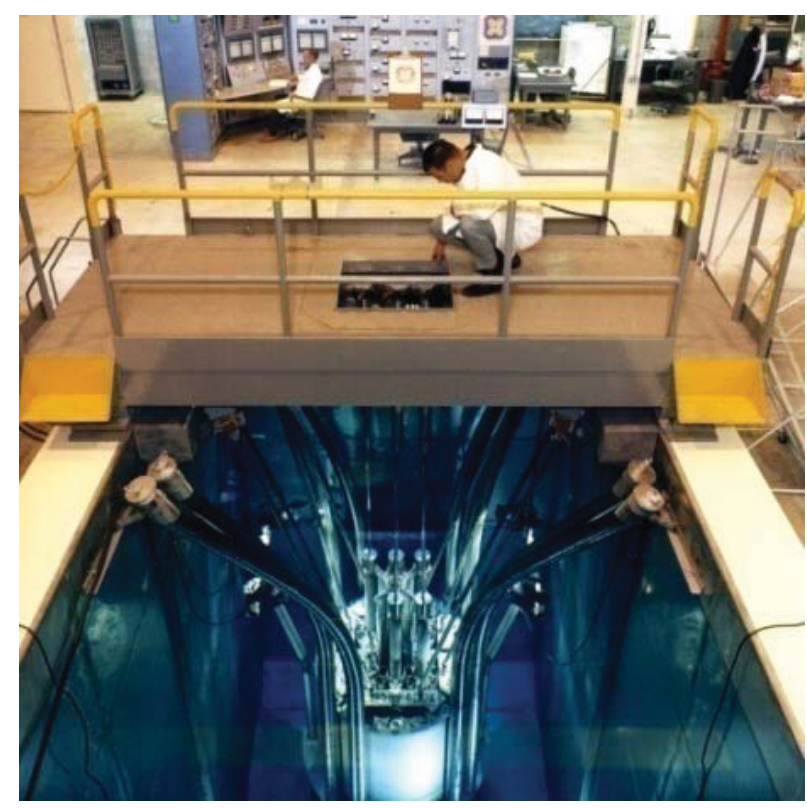

Figure 15. ATRC facility layout.

\subsubsection{Test Configurations and Conditions}

As part of the ATR National Scientific User Facility (NSUF), specialized instrument positioning hardware and associated software have been developed and installed by INL to evaluate and calibrate real-time flux sensors. Initial ATRC tests (see Figure 16), which began in 2010, are comparing the response and accuracy of specially-developed real-time flux detectors using experimental guide tubes (EGTs) that can position the detectors at up to six of the N-16 positions (with widths or diameters of approximately 0.625 inches) and specialized fixtures that can position detectors in the NorthWest Flux Trap (with a diameter of 5.25 inches). Software has been developed to process data obtained from these detectors, with the ultimate objective of providing a real-time three-dimensional ATRC flux map. Data are benchmarked with activation wire and foil measurements and modeled with reactor physics codes. The fixtures and software developed for this effort provide irradiation locations to test candidate in-pile sensors, fuels, and materials. Note that the ATRC's pool type design offers users enhanced irradiation testing flexiblity. For example, gas lines and heaters are more easily included in ATRC test configurations. 


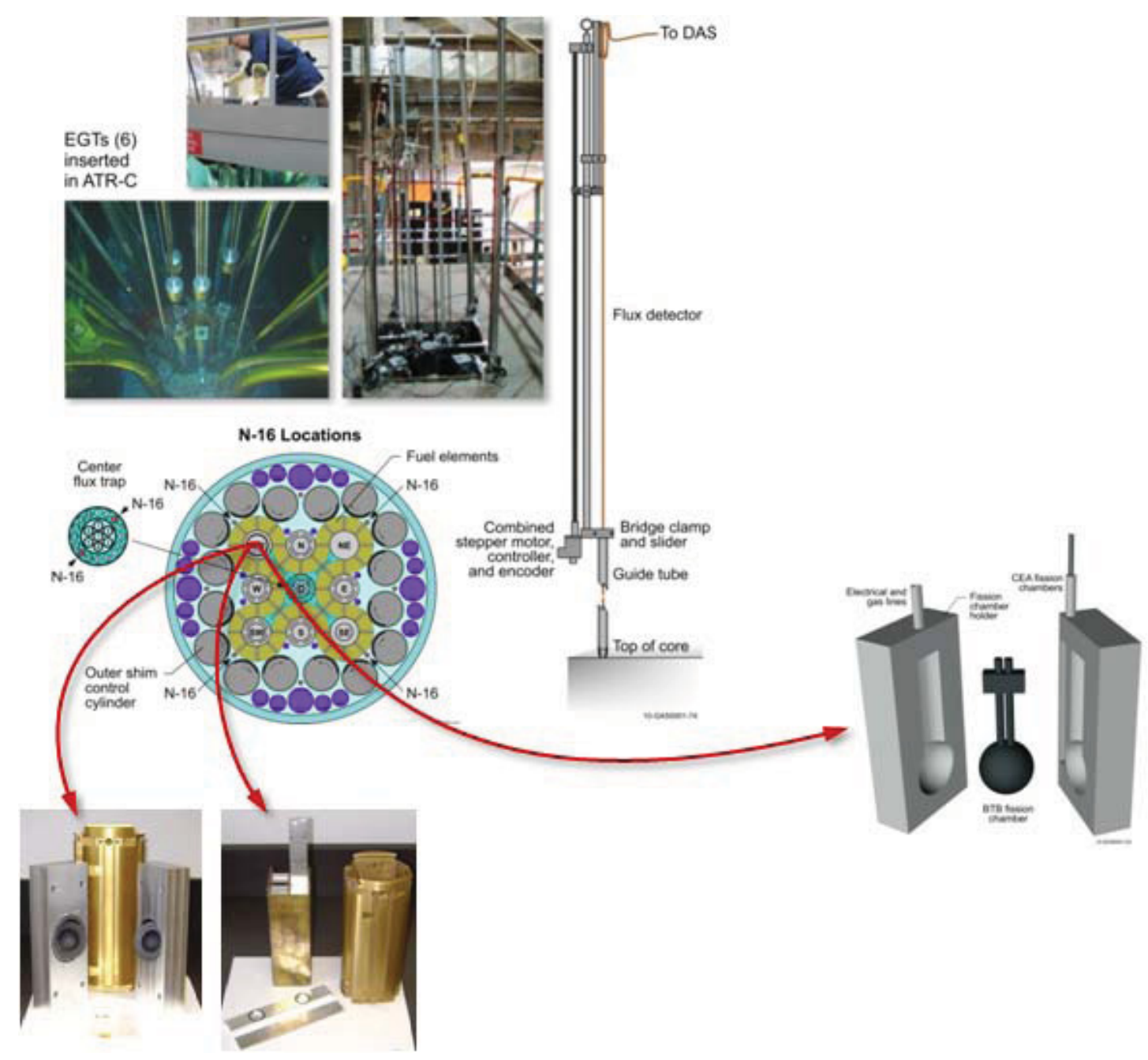

Figure 16. Sample/sensor positioning equipment and insertion locations at ATRC.

\subsubsection{Other MTRs}

Table 8 compares operating parameters for selected test and prototype reactors used (or under construction) throughout the world. Information in this table was primarily obtained from the IAEA Nuclear Research Reactor Database, ${ }^{18}$ which contains information from nearly 280 research reactors (operating, shutdown, and proposed). Although these reactors range in power levels from 0 to several hundred MWt, nearly 200 of them have power levels below $5 \mathrm{MWt}$. Most of the reactors listed in Table 8 achieved criticality in the 1960s (or earlier). Among the operating reactors, there are two exceptions: the Hanaro reactor in South Korea, which went critical in 1995 and the prototype High Temperature Test Reactor (HTTR) in Japan, which went critical in 1998. 


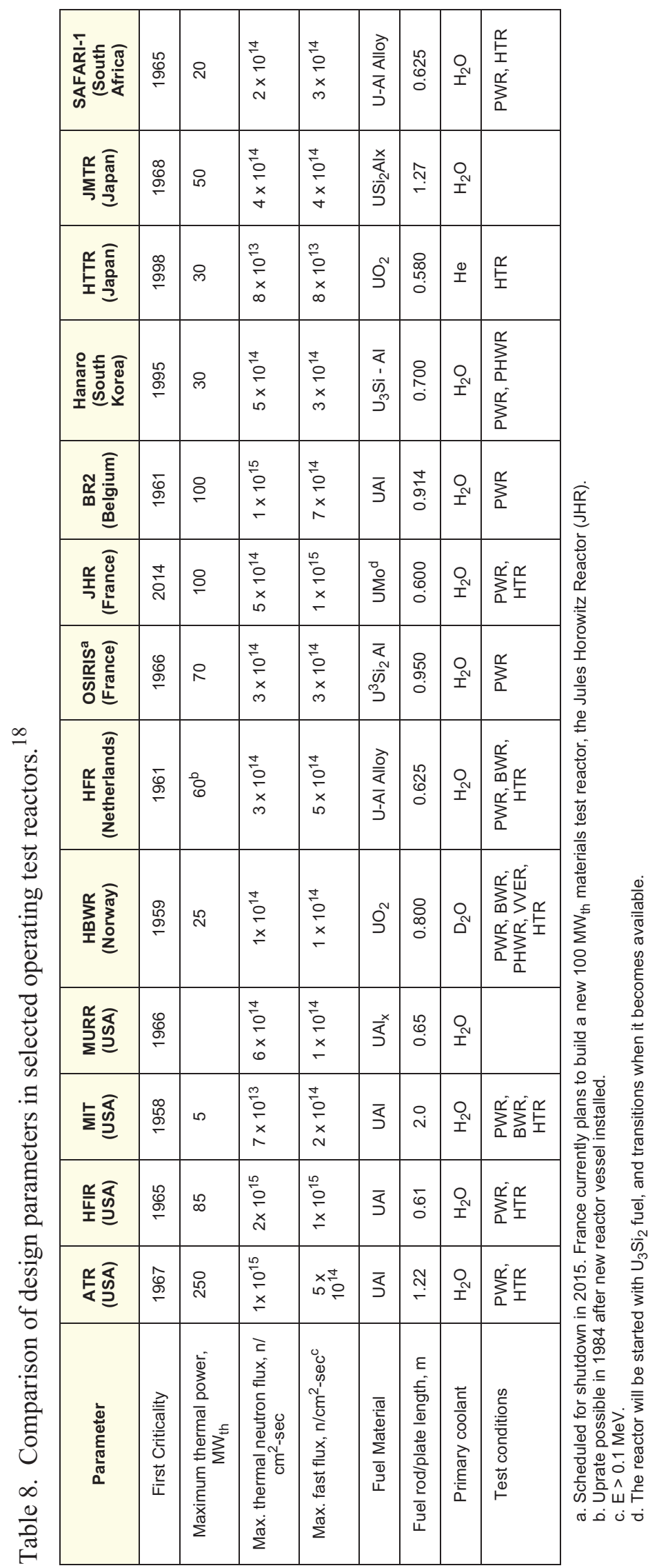


The Jules Horowitz Reactor (JHR), ${ }^{6}$ which is scheduled to go critical in 2014, is another exception with respect to initial date for achieving criticality. The JHR is being built to replace materials irradiation capabilities of older reactors in Europe as they are retired from service. This $100 \mathrm{MWt}$ reactor is designed to include static capsules, instrumented capsules, and in-pile loops. To support irradiation programs anticipated for the JHR, CEA is developing four standard types of test trains for experiments in loops at nominal and off-normal LWR conditions, of capsules at LWR accident conditions, and loops at sodium potassium loop conditions (with high dpa and low thermal gradients).

It is also worth highlighting the Halden Boiling Water Reactor (HBWR). Although this reactor is older and its maximum power level $(20 \mathrm{MWt})$ is over an order of magnitude smaller than the ATR, its testing flexibility and the expertise of its staff for instrumenting its tests make this facility unique. For decades, organizations within the international community (including the US Nuclear Regulatory Commission, vendors such as General Electric and AREVA, and the US naval reactor program) have utilized this facility for in-pile irradiation needs. Approximately $40 \%$ of HBWR testing is devoted to Organization for Economic Cooperation and Development (OECD) programs with the remainder sponsored by bilateral agreements between Norway and customers from other countries. ${ }^{11}$ Most of these bilateral agreements allow the HBWR to perform tests for utility customers to address issues related to fuel thermal performance, fuel pellet/clad interactions, fuel fission gas release, reactor vessel embrittlement, structural materials degradation (e.g., corrosion, creep, etc.). As noted in Table 8, this reactor has developed loops for simulating BWR, PWR, CANDU, and VVER conditions.

In reviewing Table 8, it is clear that the ATR and HFIR offer higher flux levels than most other test reactors. Although other test reactors, such as the HBWR, may currently have superior instrumentation capabilities and more flexible test trains, a primary goal of this effort will be to make ATR and HFIR more competitive by adding additional capabilities required for US research programs.

\subsection{Transient Test Reactors}

This section describes two representative transient test reactors: the TREAT (Transient REActor Test) facility, which is located at the Materials and Fuels Complex (MFC) of the INL and the ACRR (Annular Core Research Reactor), which is located at Technical Area V (TA-V) at the Sandia National Laboratory (SNL). As discussed in this section, these facilities differ considerably in design.

\subsubsection{TREAT}

The TREAT facility is a graphite moderated, air-cooled, thermal, heterogeneous test reactor designed to evaluate reactor fuels and structural materials under conditions that simulate various types of transient overpower and under-cooling situations in a nuclear reactor. ${ }^{17}$ TREAT first achieved criticality in 1959 and was modified and/or upgraded several times since, most recently in 1988. Throughout the reactor's operating history, 6640 reactor startups and 2884 transient irradiations were completed. The reactor was placed in standby in 1994, but it is still in excellent condition.

\subsubsection{Reactor Design and Characteristics}

The reactor core consists of highly-enriched uranium oxide fuel dispersed in a carbon and graphite matrix surrounded by a graphite reflector and a concrete shielding. The fuel and reflector assemblies are removable, allowing the core to accommodate test loops and capsules. Fuel meltdowns, metal-water reactions, thermal interaction between overheated fuel and coolant, and the transient behavior of ceramic fuel for high-temperature systems can be studied. In its steady-state mode of operation, TREAT can also be used as a large neutron-radiography facility and can examine assemblies up to 15 feet long. A schematic of the TREAT transient test facility is shown in Figure 17a, and a photograph of TREAT is shown in Figure $17 \mathrm{~b}$. 


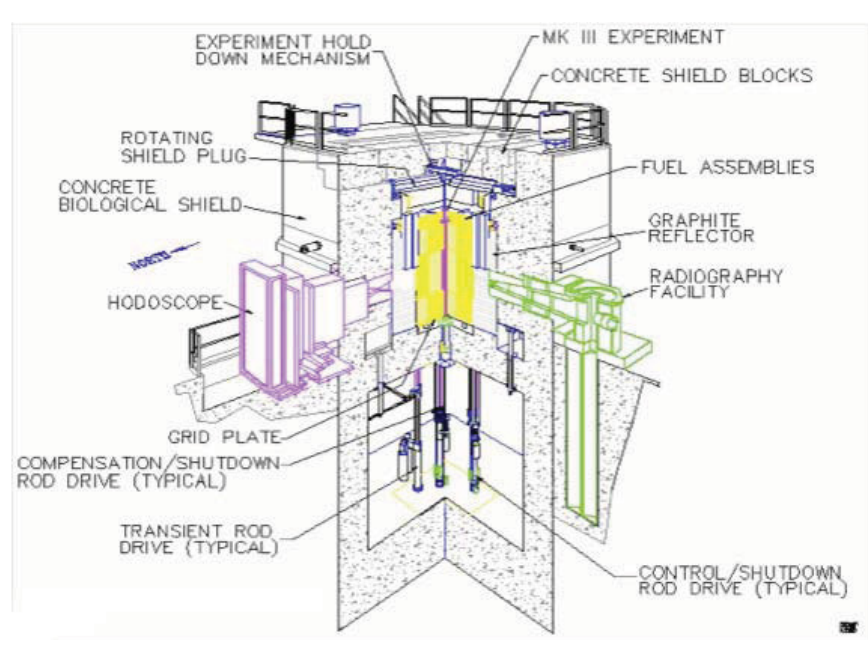

(a)

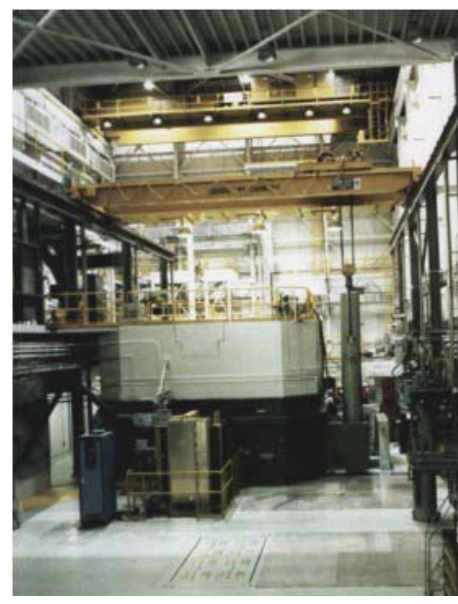

(b)

Figure 17. TREAT (a) facility schematic and (b) reactor shield (the Hodoscope is shown on the north face of the reactor, and a cask is shown on the radiography stand on the west face).

TREAT contributions to reactor safety programs include:

- providing data for predicting fuel design safety margins and the severity of potential accidents,

- serving as a proving ground for fuel design concepts to reduce or preclude the consequent hazards associated with potential accidents, and

- providing non-destructive test data through neutron radiography of fuel samples.

TREAT capabilities could add a much-needed component to advanced fuel/cladding development, modeling, and deployment.

The TREAT core is located in a concrete biological shield 5 feet thick. The shield contains numerous penetrations that can be used to support experiment and reactor operations. The core is air cooled and designed to remove the heat generated during steady-state operations or following transient operations. The core consists of a $19 \times 19$ square array of fuel and reflector assemblies. Surrounding the array is a permanent graphite reflector $2 \mathrm{ft}(0.6 \mathrm{~m})$ thick. The TREAT fuel assemblies are $4 \mathrm{in.x} 4 \mathrm{in}$. and $8 \mathrm{ft}$ long. The assemblies are made up of a $4-\mathrm{ft}(122 \mathrm{~cm})$ active fuel section, with two 2-ft axial graphite reflector sections. Experiment vehicles (e.g., loops or capsules) customarily have as many as 21 fuel assemblies. The TREAT reactor fuel is a diluted mixture of fine particles of highly enriched $\mathrm{UO}_{2}$ in graphite and carbon. The ${ }^{235} \mathrm{U}$ is approximately $0.2 \%$ by weight of the total mixture. This design permits rapid transfer of fission energy into the graphite and carbon, which results in a rapid and uniform heat up of the moderator. This process results in essentially instantaneously acting, large, negative-temperature coefficients of reactivity, and hence, self-limiting nuclear transients.

\subsubsection{Test Configurations and Conditions}

The TREAT core loading is optimized for each experiment to meet the size, reactivity, and diagnostic requirements of the experiment. The reactor is capable of developing a range of transient shapes and sizes (e.g. single burst transients that last less than a second with powers up to 19,000 $\mathrm{MW}_{\text {th }}$, shaped pulses with powers up to $10,000 \mathrm{MW}_{\text {th }}$ that last seconds, to extended transients with powers of only several $\mathrm{MW}_{\text {th }}$ that last minutes). The maximum core power and energy capabilities are dependent on administrative limits related to both the core loading and the type of transient being performed. In addition, the reactor can oper- 
ate in a steady state mode (at $100 \mathrm{~kW}$ ), which provides the neutron flux needed for neutron radiography. A number of experiment types have been designed and used in the TREAT reactor. Table 9 lists representative TREAT experiments, with corresponding power coupling factors and energy depositions.

Table 9. Examples of test-fuel power and energy generation.

\begin{tabular}{|l|l|c|c|}
\hline \multicolumn{1}{|c|}{ Fuel Type } & \multicolumn{1}{|c|}{ Power-time History } & PCF ${ }^{\text {a }}$ J/g-MJ & TED, ${ }^{\mathbf{b}}$ kJ/g \\
\hline Fast Reactor - 60 wt\% U-235 in Fuel (no Pu) & Shaped transient & 5 & 9 \\
\cline { 2 - 4 } & Natural burst & 3 & 43 \\
\hline Fast Reactor - 30 wt\% Pu in HM, Natural U & Shaped transient & 0.9 & 1.2 \\
\hline PWR - 5\% enriched, 80 GWd/MTM & Natural burst - FWHM ${ }^{\mathrm{c}}$ 65-70 msec & 0.9 & 0.4 \\
\cline { 2 - 4 } & Natural burst - FWHM 55-60 msec & 0.5 & 1.0 \\
\hline CANDU - Natural U, Zero Burnup & RIA pulse - FWHM of 1 to 2 sec & Not applicable & 3 \\
\hline PWR - 8\% enriched, 35 GWd/MTM & Extended transients of 20 minutes & \multicolumn{2}{|c|}{3} \\
\hline
\end{tabular}

a. Power Coupling Factor (PCF) - ratio between fission power generated in test fuel and power generated in TREAT core [expressed in units of watts per gram of test fuel per MW of TREAT power (W/g-MW), or equivalently, in units of joules per gram of test fuel per MJ of TREAT energy $(\mathrm{J} / \mathrm{g}-\mathrm{MJ})]$.

b. Total fission Energy Deposition (TED) in test fuel. The product of the PCF times the total core energy generated.

c. FWHM- Full width at half maximum (of pulse).

Flowing-coolant loops (for prototypic, multiple-effects, complex interaction tests) were typically used in the TREAT reactor. TREAT coolant loops (containing sodium, water, steam, and inert gas) include:

- Recirculating coolant ("package" style, or with part of loop outside the core)

- Once-through coolant (most of loop outside the core)

- Capsules, (for "phenomenological," "separate-effects," basic process tests)

- $\quad$ Gas-filled (dry)

- Stagnant liquid coolant

- Other configurations (e.g., for experiments with no reactor fuel).

Figure 18 shows a schematic depiction of two flowing coolants loops designed for TREAT, a flowing sodium coolant loop, and a steam recirculating loop.
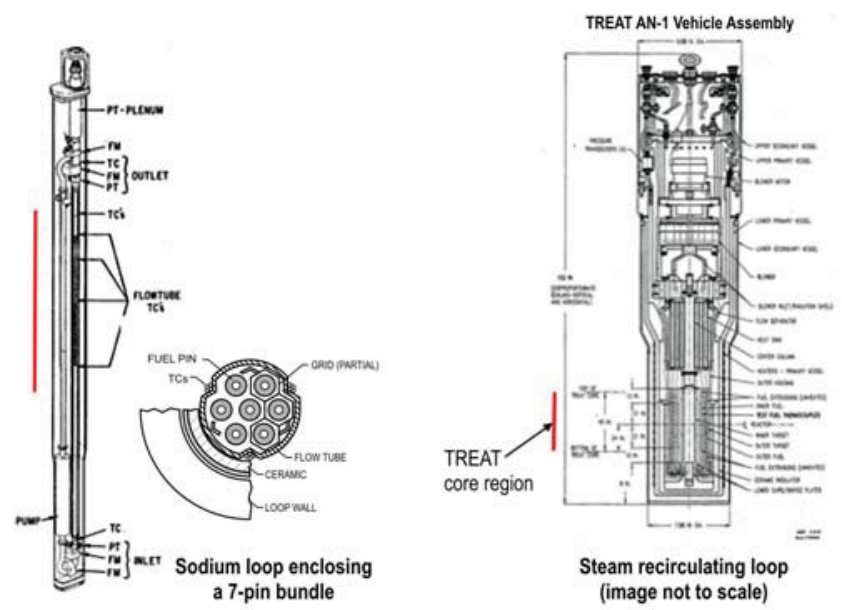

Figure 18. Representative TREAT experimental loops. 
Because TREAT core elements (fueled elements, un-fueled graphite elements, slotted elements, etc.) were easily moved in and out of the core, the core was loaded to accommodate a variety of sizes and shapes of experimental assemblies. The largest test vessel run in TREAT occupied 214 -in. ${ }^{2}$ fuel positions, and the smallest occupied a single fuel position. Many of the test vehicles that were used in TREAT can test more than one fuel pin simultaneously (e.g., in multi-pin bundles or with pins in separate flowtubes). For example, the Mark-III sodium loops, which were high-pressure stainless steel vessels and occupied two fuel positions, were capable of testing up to seven LMFBR-type fuel pins. Large areas for experiment hardware exist on top of the reactor, on the reactor building floor near the reactor, or in the mezzanine area of the reactor building adjacent to the top of the reactor. A variety of utility services are available for experiment support, including electrical power sources, cooling water, and gas systems.

TREAT has a long history of successful testing of a range of fuel types. A spectrum of fast reactor fuels was tested (both oxide and metal), and tests were also conducted on a variety of thermal reactor fuels. The power deposition achievable in experimental fuels will vaporize fuels, but most of the tests were conducted to better understand the progression of fuel failure under severe transient conditions. Accordingly, a range of transient conditions, power deposition, and peak temperatures was explored. One of the more important issues for new fuels is how well fuel behavior is understood for high-temperature conditions at the threshold of failure; tests previously completed in TREAT addressed this issue.

High quality data collection is important to understanding behavior under such severe conditions and several novel instruments were developed at TREAT for this purpose. At TREAT, the neutron Hodoscope (fuel motion monitoring system) provided real-time imagery of the movement of fuel during experiments. Figure 19 provides top-view and side-view schematics of the hodoscope system. The hodoscope measured the test fuel motion experienced during the conduct of a transient experiment. Figure 20 provides an example of the type of information that was generated from the analysis of hodoscope data. In the figure, the successive images show the time progression of fuel density in each hodoscope pixel in a test in which two fuel pins were located side by side. The pin on the left side, which did not fail during the test, was aligned with a column of hodoscope pixels; the pin on the right side, which did fail, was located in the area viewed by two adjacent columns. In general, the mass resolution obtained using the hodoscope is, at best, 0.2 grams of fuel per channel for single-pin experiments and 0.8 grams of fuel per channel for 7-pin experiments.

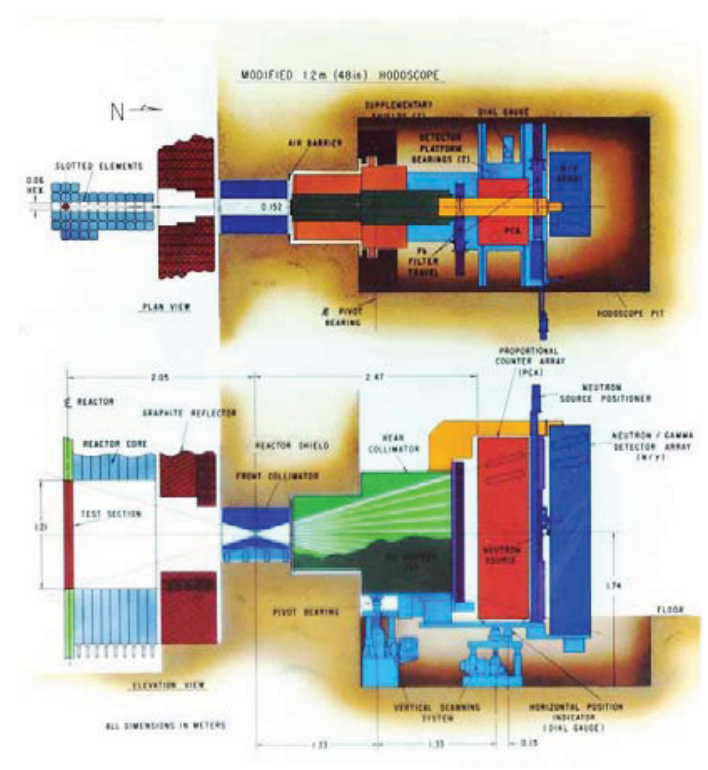

Figure 19. Schematic of the TREAT hodoscope. 

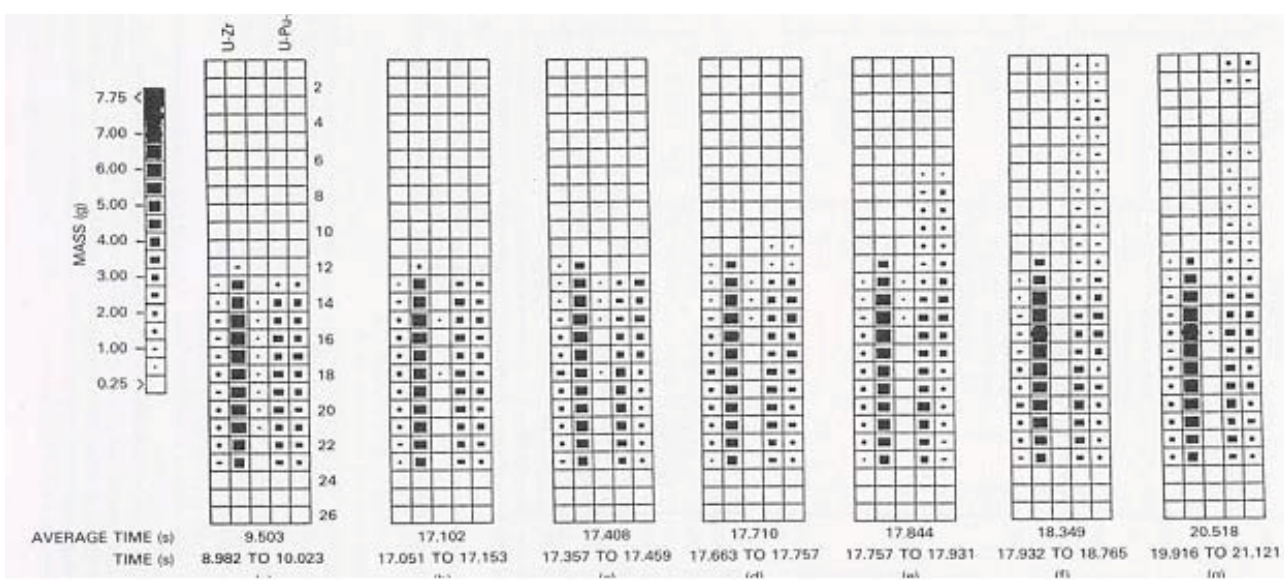

Figure 20. Example of analyzed TREAT hodoscope data (fuel motion with time).

Three multi-channel neutron collimators are also available at TREAT. The one most often used has a viewing region at the core center of $66 \mathrm{~mm}$ (10 pixels) wide $\times 1200 \mathrm{~mm}$ (36 pixels) high and provides spatial resolution as low as $0.2 \mathrm{~mm}$ horizontally and $1.0 \mathrm{~mm}$ vertically. Smaller and larger collimators are also available. The neutron radiography facility alongside the reactor can accommodate most types of experiment vehicles that have been used. Data acquisition capabilities are also available. High-resolution neutron radiography also exists at the nearby Hot Fuel Examination Facility, where experiment vehicles (loops or capsules) may be assembled and disassembled, and where metallography/ceramography can be performed.

The computer components of the TREAT Hodoscope and experimental data acquisition systems are old. Although functional, they no longer have adequate vendor support. If TREAT is restarted, an effort will be needed to improve instrumentation and data collection systems using technology advances during the last two decades and exploring other instrumentation options, such as tomography. Such an effort could significantly enhance the value of TREAT for fuel cycle research and development.

\subsubsection{ACRR}

The ACRR is a pulse and steady-state pool-type reactor that maintains a large, dry irradiation cavity at the center of its core. The ACRR is typically used to perform irradiation testing where a high neutron flux is required for a short period of time. Historically, the ACRR has been used for a wide variety of experiment campaigns including weapons effects testing, nuclear fuels testing, nuclear pumped laser experiments, space nuclear thermal propulsion testing, and medical isotopes production. With regard to fuels testing, the ACRR has been used to investigate fuel pin design limitations and clad and fuel relocation for the fast reactor safety program, and severe fuel damage and fission product release for the light water reactor program. Currently, the ACRR is fully operational. Its main attributes include a large, dry irradiation cavity, epithermal neutron flux, and large pulsing capabilities.

\subsubsection{Reactor Design and Characteristics}

The ACRR, in its current configuration, was assembled in 1978 to accommodate large experiments at the center of its core and have large pulsing capabilities. The fuel elements for the ACRR are similar in size and shape to TRIGA fuel. However, the fuel is unique in that the fuel meat is $\mathrm{UO}_{2}-\mathrm{BeO}$ that was specially designed for a large heat capacity and, thus, larger pulsing capabilities. Figure 21 shows the ACRR looking into the pool during a $2 \mathrm{MW}$ steady-state power operation. The ACRR is shown on the left in the figure. The 9-inch-diameter dry cavity extends from above the pool through the center of the core. The reactor facility also accommodates the fueled ring external cavity-II (FREC-II), shown on the right in the 
figure, which maintains a larger dry cavity (20-inch diameter) and uses U-ZrH TRIGA fuel as a subcritical multiplier.

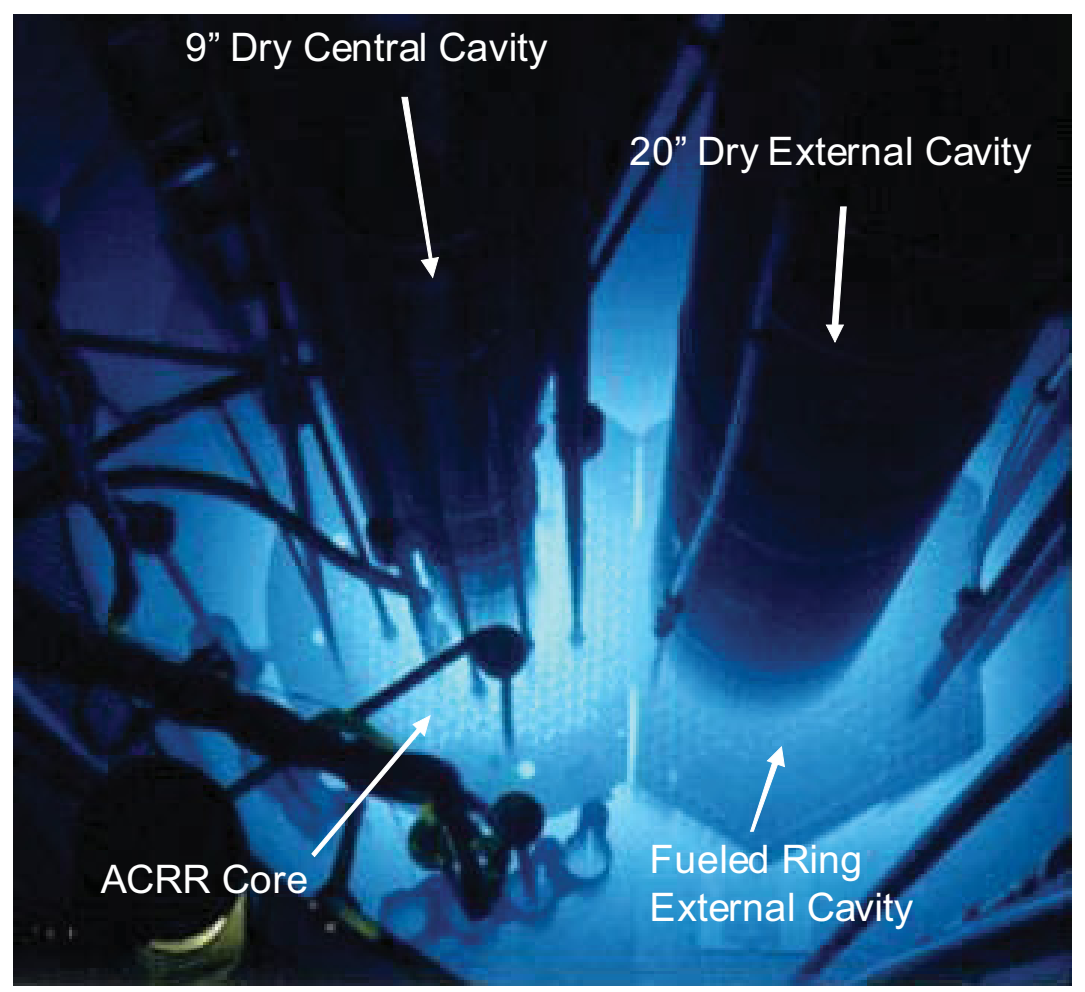

Figure 21. ACRR and FREC-II operating at $2 \mathrm{MW}$ steady-state power.

Figure 22 shows an MCNP model depiction of the ACRR and dry central cavity. The ACRR fuel elements are stainless steel clad, 1.5 inches in diameter and 21 inches in length. Within the elements are niobium cups that hold the $\mathrm{UO}_{2}-\mathrm{BeO}$ fuel pieces. The dry central cavity extends from above the pool and goes directly through and below the core region. The ACRR maintains an epithermal neutron flux spectrum in the core and central cavity. This allows for the neutron energy flux spectrum to be tailored to the desired specifications of the experiment. Moderators can be used within the cavity to thermalize the neutron spectrum. Boron and lead can be used to increase the fast neutron flux ratio and decrease the gamma-ray flux, respectively. For an unmoderated condition, the neutron flux at the center of the central cavity, at the core axial centerline is $\sim 4 \times 10^{13} \mathrm{n} / \mathrm{cm}^{2}$-s at a steady-state reactor power level of $2 \mathrm{MW}$. About $45 \%$ of the neutron flux is above $100 \mathrm{keV}$ and $56 \%$ above $10 \mathrm{keV}$.

The ACRR can operate in a steady-state, transient, or pulse mode. In the steady-state mode, the operating power level is limited to $2 \mathrm{MW}$. In the pulse mode, a maximum pulse size of $250 \mathrm{MJ}$ with a full-width half-maximum of $6 \mathrm{~ms}$ can be attained. In the transient mode, the reactor power shape can be tailored to the desired requirements for a total reactor energy deposition of 300 MJ. For example, the reactor power can be rapidly raised from $1 \mathrm{~kW}$ to $20 \mathrm{MW}$ and held there for $\sim 15$ seconds. Other scenarios include attaining a high power level for a few seconds followed by a ramp increase in power, or a ramp from low power to high power. The transient operating mode allows for a high degree of flexibility in power shaping to meet the desired experimental conditions.

The coupling factor is defined as the amount of fission energy that can be produced in a fissile experiment per gram of fissile fuel and per MJ of reactor power. The coupling factor for an unmoderated cavity is $\sim 18$ $\mathrm{J} / \mathrm{g}-\mathrm{MJ}$, and $\sim 90 \mathrm{~J} / \mathrm{g}-\mathrm{MJ}$ for the moderated condition. These coupling factors allow for fueled experiments to be designed for a wide variety of test conditions for steady-state, transient, and pulse operations. 


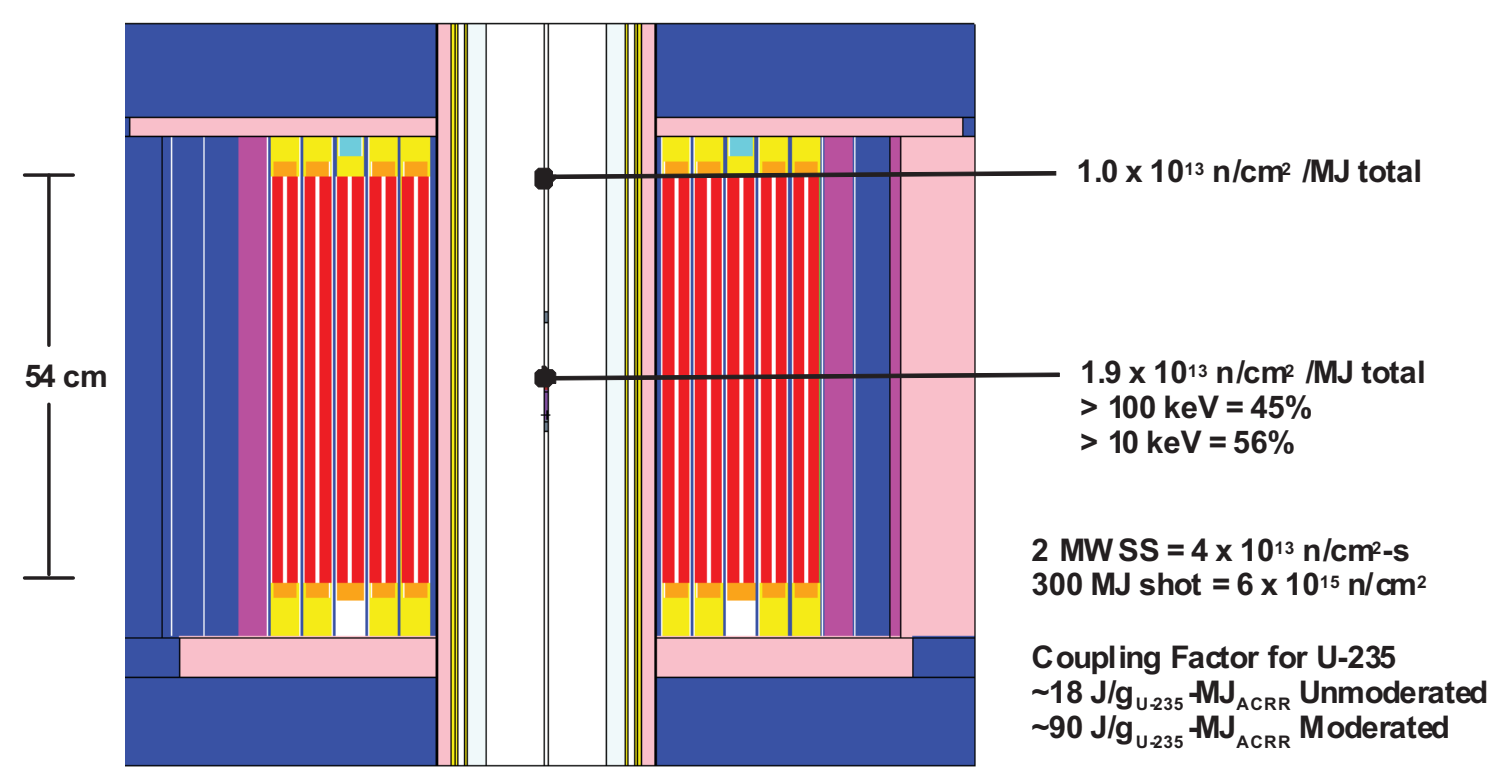

Figure 22. Cross-sectional view of the ACRR as modeled in MCNP.

\subsubsection{Test Configurations}

The ACRR central cavity allows for a high degree of experiment flexibility, along with in-situ and real-time experiment instrumentation and diagnostics. The large size of the cavity allows for the possibility of flow loops and other complex experiment hardware to be fielded within the high-flux region of the core. For fueled experiments, an experiment containment is required that is typically a cylindrically sealed capsule with instrument lead penetrations. For experiments without fuel, instrumentation can be inserted directly into the cavity using a simple experiment stand. This may be useful, as new diagnostic and measurement techniques are developed, to determine their survivability and operating characteristics in a neutron and gamma reactor environment. Approximately 30 feet of instrumentation cabling is required to go from the cavity core centerline to the reactor floor.

Figure 23 shows experiment capsules for single and multiple fuel rodlet test. The left view shows an experiment capsule nine inches in diameter, used for video recording a fuel rodlet as its design limits are exceeded. A quartz window in the top lid allows for video recording above the cavity. A similar capsule can be used with advanced diagnostics to measure strain, temperature, pressure, neutron flux, and/or other variables. The right view shows a capsule with rodlets at different heights, allowing for different fuel power conditions due to the axial flux variation in the central cavity. The plot shows the clad maintained at a constant temperature as each fuel rodlet achieves a different maximum temperature. Using this approach, all of the rodlets experience the same neutron flux time profile during the operation.

Many other types of experiment configurations can be envisioned for testing in the ACRR central cavity. Since the cavity extends the full length of the pool, the experiment package geometry is limited to nine inches in diameter by about eight feet in length. Gas, water, or liquid metal cooling of the fueled experiment can be incorporated into the experiment capsule. For experiments requiring additional external cooling, a heat exchanger with external water or gas flow can be incorporated into the experiment. If larger-diameter experiment capsules are required, the FREC-II 20-inch-diameter cavity can be used that maintains a somewhat lower magnitude neutron flux. 

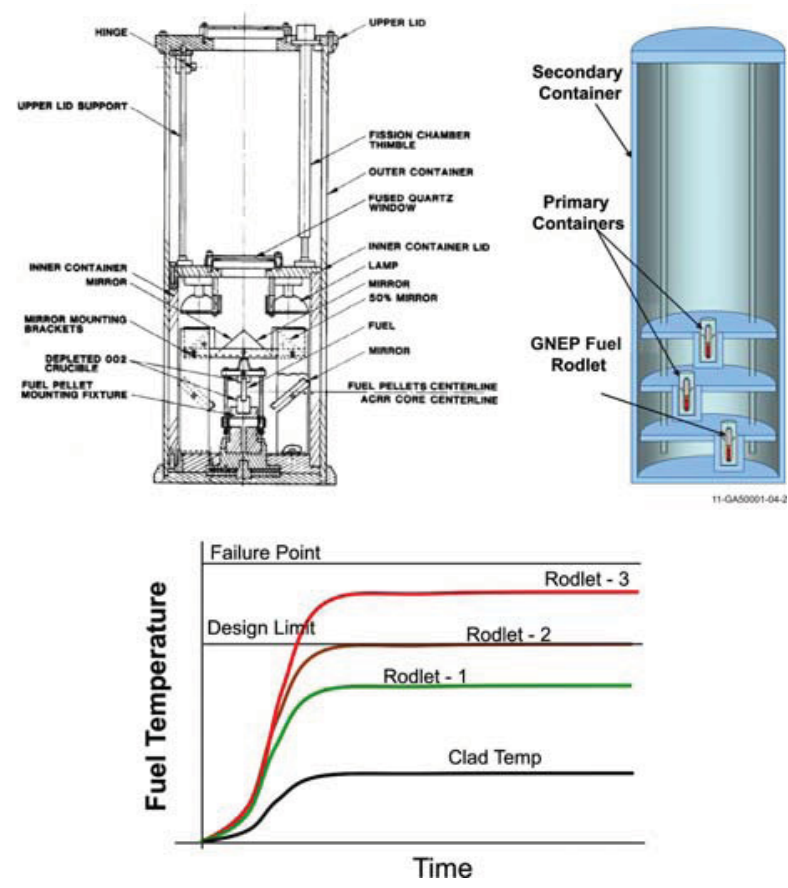

Figure 23. Experiment configuration for a single or multiple fuel rodlets.

Auxiliary facilities exist in TA-V to provide support for experiments conducted at the ACRR. These facilities include capabilities for DOT container package receiving and shipping; experiment capsule preparation, handling, and storage; and destructive and non-destructive post-radiation examination. A team of highly trained operators, technicians, and experimenters exist for the ACRR and other facilities in TA-V to provide support for both simple and complex experiment campaigns.

\subsubsection{Other Transient Test Options}

Of other possible options for transient testing, Reference 17 emphasizes two operating transient test reactors: CABRI in Cadarache, France and the NSRR in Japan. Similar to the ACRR, these test reactors also have water-cooled loops, which limit the magnitude and speed of possible transients. Although references suggest that instrumentation available at these facilities may be more precise than sensors available when TREAT last operated, the test volumes in these other facilities are too small to accommodate a full fuel assembly, and the neutron hodoscope was only available at TREAT. Furthermore, the possible loop conditions, test sizes, and test durations do not match capabilities of TREAT.

\subsection{Accelerator/Neutron Sources}

Recognizing the difficulties associated with obtaining data with the required accuracy for the FCR\&D effort from test reactors, two representative options for exposing candidate fuels to neutron fluxes are described in this document along with a discussion of the difficulties associated with deploying each option.

\subsubsection{SNS}

The Spallation Neutron Source (SNS) is an accelerator-based neutron source in Oak Ridge, Tennessee, that provides the most intense pulsed neutron beams (e.g. fast fluxes of $8 \times 10^{7} \mathrm{n} / \mathrm{cm}^{2}$-s and thermal fluxes of $\sim 1 \times 10^{16} \mathrm{n} / \mathrm{cm}^{2}$-s) in the world for scientific and industrial research and development. ${ }^{19}$ The capabilities of SNS allow for measurements of greater sensitivity, higher speed, higher resolution, or in more complex sample environments than have been possible at other existing neutron facilities. As indicated in Figure 24, 
SNS has a suite of 25 instruments either planned, under design or construction, or in operation, that are capable of detecting changes in fuels and material structures when (e.g., down to $10^{-9} \mathrm{~m}$ in some cases) when exposed to neutrons.

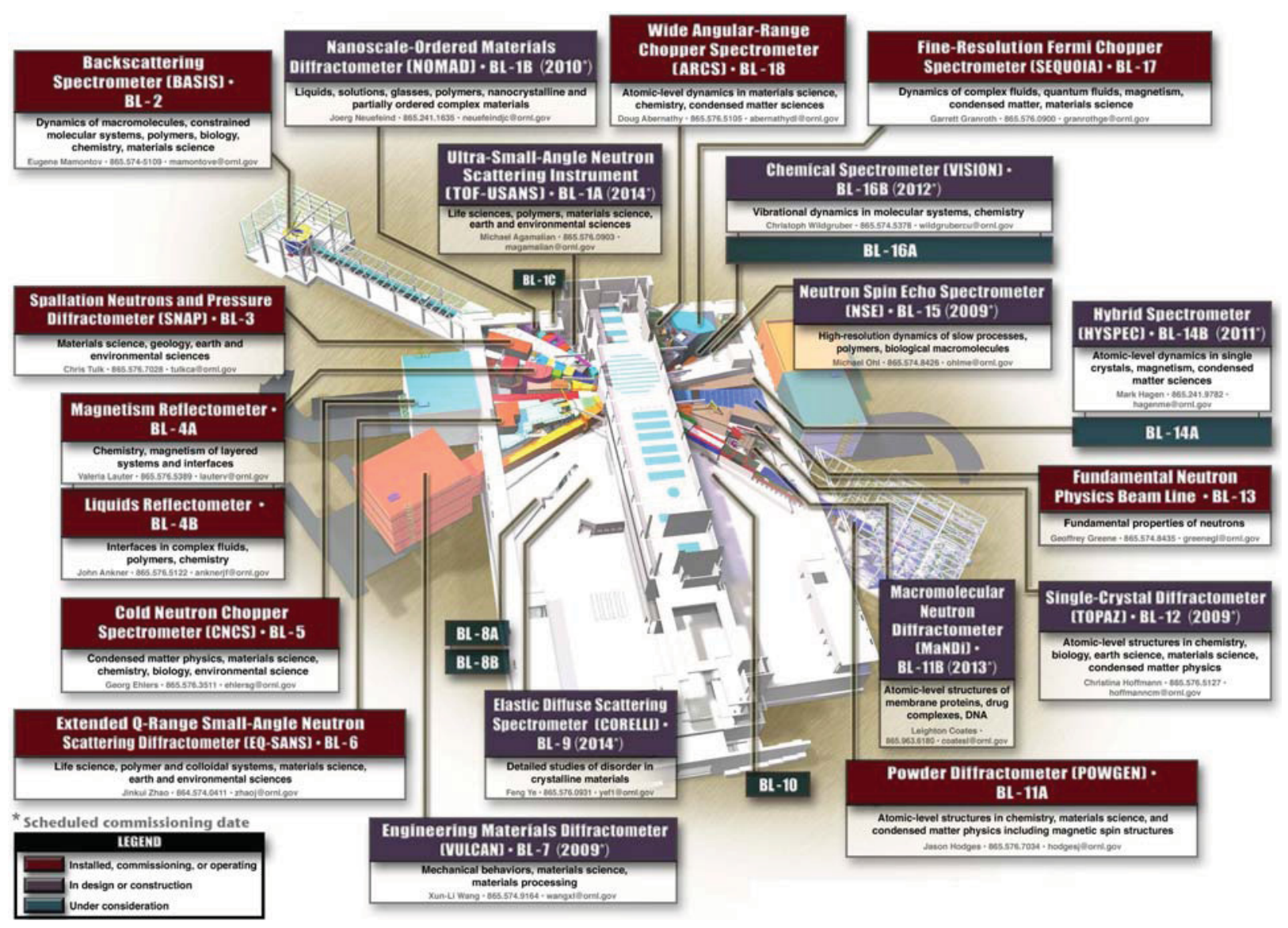

Figure 24. SNS instrumentation currently available or under investigation. ${ }^{19}$

\subsubsection{INSIGHTS}

Reference 20 presents a conceptual design for a new Intense Neutron Spectra with Independent Gamma, Hydraulic and Temperature Separate-effects (INSIGHTS) facility with independent gamma delivery, hydraulic application and temperature control to provide an experimental environment with increased flexibility, control and access for detailed measurements on small fuel samples and configurations. This facility would be designed to provide a number of direct line-of-sight experimental channels capable of delivering tailored neutron spectra with fast fluxes that approach $10^{12} \mathrm{~cm}^{-2} \mathrm{~s}^{-1}$, with prompt gamma radiation highly suppressed by the lead scattering media. An adjustable electron linear accelerator would be used as an external gamma source, controlled independently of the neutron flux, to provide specific doses to any of the experimental channels. The inclusion of small-scale ovens and hydraulic presses in experimental volumes will provide the other independently controlled forces known to impact early fuel dynamics. Reference 20 also proposes that advanced measurement techniques be developed to deliver the specific and detailed data required to benchmark and advance the fuels modeling and simulation effort.

The INSIGHTS system (the "baseline" configuration is shown in Figure 25) will be designed to provide experimental conditions and access to samples irradiated in a controlled environment. At the heart of the system will be a novel, powerful neutron source (in yellow). The neutron generator will be able to run in a continuous or pulsed mode. The lead scattering chamber (in gray), which is approximately $3 \mathrm{~m} \times 3 \mathrm{~m}$, will 
increase the density of neutrons and slightly moderate the flux shape through series of elastic collisions will surround the source. A number of experimental channels will be designed to provide highly flexible configurations with unprecedented access. The experimental packages (in pink and blue) are loaded into the primitive bores and then capped with lead inserts (light grey), which could also be instrumented as needed. LEU pins can also be used to harden the spectrum and provide fast flux boosting to nearly $10^{15} \mathrm{~cm}^{-2} \mathrm{~s}^{-1}$. The simplicity of the system will significantly reduce modeling uncertainties that arise from complex environmental simulation. The INSIGHT system will also utilize a linear accelerator to deliver prescribed doses of gamma rays to an experiment and a heating system to maintain experiments at the desired temperature.

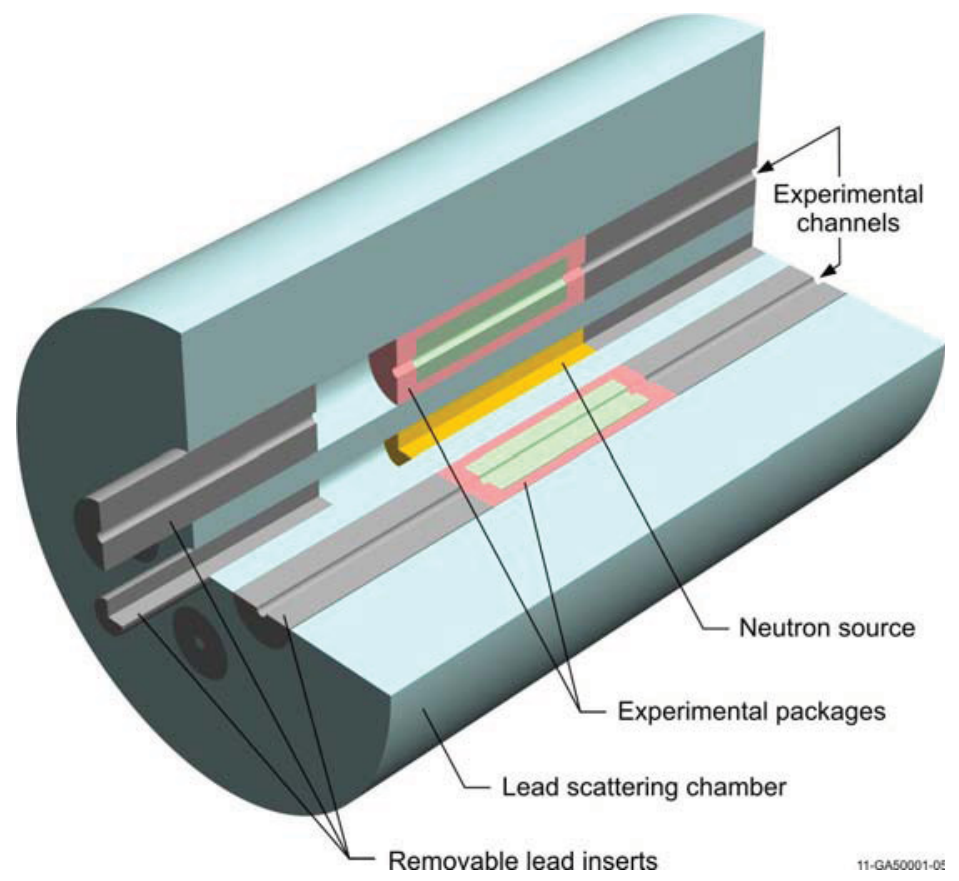

Figure 25. INSIGHTS conceptual design showing neutron source (yellow) surrounded by lead scattering chamber (grey).

\subsection{Summary}

As described in this section, there are several irradiation options available to support FCR\&D. A comprehensive evaluation of fuel performance should include irradiations in a test reactor (with elevated flux levels to accelerate fuel damage expected during normal operation) and in a transient test facility (to simulate performance during accident conditions). As discussed in Section 2, experimental data indicate that most fuel damage is incurred during the first 24 hours of irradiation. Hence, irradiation testing in a more accessible, lower flux-level facility, such as the ATRC or a neutron accelerator, might provide key insights.

Clearly, each type of irradiation option described in this section offers specific advantages. Ultimately, a comprehensive effort that included transient testing and testing in neuron source facilities would be advantageous. For such a case, enhanced sensors would be needed in each reactor/irradiation location to extract the required data with the required precision. However, this FCR\&D effort will initially focus on advanced instrumentation for the ATR and HFIR, which are currently used by the FCR\&D program. However, the applicability of sensors developed in this effort to other irradiation facilities, such as transient test facilities, will be considered. 


\section{IN-PILE INSTRUMENTATION}

ATR and HFIR irradiation capabilities in static capsule, shuttle, instrumented lead, and PWR loop tests (only ATR), are unparalleled. However, as indicated in column 5 of Table 10, available instrumentation for detecting parameters of interest to the FCR\&D program is limited at each irradiation location. ${ }^{5}$ As indicated by column 6 , sensors available at other MTRs could increase detection capabilities during ATR and HFIR irradiations. Furthermore, as indicated by column 7, there are several "Developmental" technologies that could also be explored for in-pile applications.

Table 10. Instrumentation available and proposed advanced technologies.

\begin{tabular}{|c|c|c|c|c|c|c|}
\hline \multirow[b]{2}{*}{ Parameter } & \multicolumn{3}{|c|}{ Location } & \multirow[b]{2}{*}{$\begin{array}{l}\text { Available Technology } \\
\text { for ATR and HFIR }\end{array}$} & \multicolumn{2}{|c|}{ Proposed Advanced Technology } \\
\hline & $\begin{array}{c}\text { Static } \\
\text { Capsule/ } \\
\text { Shuttle }\end{array}$ & $\begin{array}{l}\text { Instr. } \\
\text { Lead }\end{array}$ & $\begin{array}{l}\text { PWR } \\
\text { Loop }\end{array}$ & & Available at Other MTRs & Developmental \\
\hline \multirow[t]{2}{*}{ Temperature } & $\checkmark$ & $\checkmark$ & $\checkmark$ & $\begin{array}{l}\text {-Melt wires (peak) } \\
\text {-SiC Temperature } \\
\text { Monitors (range) }\end{array}$ & -Paint spots (peak) & - Wireless $^{\mathrm{a}}$ \\
\hline & & $\checkmark$ & $\checkmark$ & $\begin{array}{l}\text {-Thermocouples (Type } \\
\mathrm{N}, \mathrm{K}, \mathrm{C}^{\mathrm{b}} \text {, and } \\
\text { HTIR-TCs) }\end{array}$ & -Expansion Thermometer & $\begin{array}{l}\text { - Fiber Optics } \\
\text {-Noise Thermometry } \\
\text {-Ultrasonic Thermometers }\end{array}$ \\
\hline $\begin{array}{l}\text { Thermal } \\
\text { Conductivity }\end{array}$ & & $\checkmark$ & $\checkmark$ & $\begin{array}{l}\text {-Out-of-pile } \\
\text { examinations }\end{array}$ & $\begin{array}{l}\text {-Degradation using signal } \\
\text { changes in thermocouples }\end{array}$ & -Hot wire techniques \\
\hline \multirow{2}{*}{$\begin{array}{l}\text { Flux/Fluence } \\
\text { (neutron) }\end{array}$} & $\checkmark$ & $\checkmark$ & $\checkmark$ & -Flux wires (Fe, Ni, Nb) & -Activating foil dosimeters & \\
\hline & & $\checkmark$ & $\checkmark$ & & $\begin{array}{l}\text {-Self-Powered Neutron } \\
\text { Detectors (SPNDs) } \\
\text {-Subminiature / miniature } \\
\text { fission chambers }\end{array}$ & \\
\hline $\begin{array}{l}\text { Gamma } \\
\text { Heating }\end{array}$ & & $\checkmark$ & $\checkmark$ & & $\begin{array}{l}\text {-Degradation using signal } \\
\text { changes in thermocouples }\end{array}$ & \\
\hline \multirow[t]{2}{*}{ Dimensional } & $\checkmark$ & $\checkmark$ & $\checkmark$ & $\begin{array}{l}\text {-Out-of-pile } \\
\text { examinations }\end{array}$ & & \\
\hline & & $\checkmark$ & $\checkmark$ & & $\begin{array}{l}\text {-LVDT-based elongation } \\
\text { (stressed and unstressed) } \\
\text {-Diameter gauge } \\
\text {-Hyper-frequency resonant } \\
\text { cavities }\end{array}$ & $\begin{array}{l}\text {-Ultrasonic Transducers } \\
\text {-Fiber Optics }\end{array}$ \\
\hline $\begin{array}{l}\text { Fission Gas } \\
(\text { Amount, } \\
\text { Composition) }\end{array}$ & & $\checkmark$ & $\checkmark$ & $\begin{array}{l}\text {-Gas Chromatography } \\
\text {-Pressure sensors } \\
\text {-Gamma detectors / } \\
\text { Sampling }\end{array}$ & $\begin{array}{l}\text {-LVDT-based pressure } \\
\text { monitors } \\
\text {-Counter-pressure monitor } \\
\text {-Sampling ('near' real-time) }\end{array}$ & $\begin{array}{l}\text {-Acoustic measurements } \\
\text { with high-frequency } \\
\text { echography }\end{array}$ \\
\hline Loop Pressure & & & $\checkmark$ & $\begin{array}{l}\text {-Differential pressure } \\
\text { transmitters } \\
\text {-Pressure gauges with } \\
\text { impulse lines }\end{array}$ & & \\
\hline Loop Flowrate & & & $\checkmark$ & $\begin{array}{l}\text {-Flow venturis } \\
\text {-Orifice plates }\end{array}$ & & \\
\hline $\begin{array}{l}\text { Loop Water } \\
\text { Chemistry }\end{array}$ & & & $\checkmark$ & $\begin{array}{l}\text {-Off-line sampling / } \\
\text { analysis }\end{array}$ & $\begin{array}{l}\text {-Electrical chemical potential } \\
\text { probes }\end{array}$ & \\
\hline $\begin{array}{l}\text { Crud } \\
\text { Deposition }\end{array}$ & & & $\checkmark$ & $\begin{array}{l}\text {-Out-of-pile } \\
\text { examinations }\end{array}$ & $\begin{array}{l}\text {-Diameter gauge with } \\
\text { neutron detectors and } \\
\text { thermocouples }\end{array}$ & \\
\hline $\begin{array}{l}\text { Crack Growth } \\
\text { Rate }\end{array}$ & & & $\checkmark$ & $\begin{array}{l}\text {-Out-of-pile } \\
\text { examinations }\end{array}$ & $\begin{array}{l}\text {-Direct Current Potential } \\
\text { Drop (DCPD) technique }\end{array}$ & \\
\hline
\end{tabular}

a. Although listed under temperature, wireless technologies could be pursued for many parameters

b. Type C thermocouple use requires a "correction factor" to correct for decalibration during irradiation. 
As part of the ATR National Scientific User Facility (ATR NSUF) program, several activities have been initiated to develop and implement new in-pile instrumentation capabilities. Blue text in Table 10 indicates current instrumentation research efforts, and red text indicates new sensors now available to users as a result of recent instrumentation research. The instrumentation currently being developed was selected based on anticipated ATR NSUF user needs and 'technology readiness' (allowing this program to provide users needed instrumentation in the near-term). Many of these instrumentation development efforts are in collaboration with other organizations. As indicated in Table 10, two new sensors are now available to ATR users as a result of this instrumentation development effort. New sensors available for ATR users that can detect all of the parameters listed in Table 10 is the ultimate goal of this ATR NSUF effort.

\subsection{Temperature}

As indicated in Table 10, available temperature detection sensors are comparable, if not superior, to those used at other MTRs. Melt wires and temperature-sensitive paint spots, along with commercially available thermocouples (e.g., Types K, N, and C) have been implemented into MTR tests for decades. To meet recent customer requests, two new techniques have been implemented: the use of SiC temperature monitors in static capsules and the use of doped molybdenum/niobium alloy for instrumented lead and PWR loop applications.

\subsubsection{Static Capsule Tests}

For test capsules without any instrumentation leads, temperature detection is limited to melt wires and $\mathrm{SiC}$ temperature monitors that can detect peak irradiation temperature. Capabilities of these sensors are described below.

Melt wires. Metal wires of a known composition and melting temperature are placed in an environment to determine if a specific peak temperature is reached. Note that a post-test examination of the wire is required to determine if melting actually occurred indicating that the corresponding melting temperature was reached (or exceeded). As described in ASTM E 1214-06, ${ }^{21}$ melt wire materials should consist of metals with $99.9 \%$ purity or be eutectic alloys such that their measured melting temperatures are within $\pm 3{ }^{\circ} \mathrm{C}$ of the recognized melting temperatures. Transmutation-induced changes of these wires should not be considered significant up to $1 \times 10^{20} \mathrm{n} / \mathrm{cm}^{2}(\mathrm{E}>1 \mathrm{MeV})$. As noted in Reference 21, melt wires should be selected to measure temperature at 5 to $12{ }^{\circ} \mathrm{C}$ intervals, with at least one melt wire that possesses a melting temperature greater than the highest anticipated temperature. Melt wires are often encased in vanadium or quartz containers. As part of the ATR NSUF effort, INL has developed in-house capabilities to verify the melting temperature of candidate wire materials and to encase multiple types of melt wires into a single small diameter unit for irradiation testing.

Silicon Carbide Temperature Monitors. In recent years, several research organizations have explored the use of $\mathrm{SiC}$ temperature monitors for static capsule peak temperature detection because these monitors can be used to detect the maximum temperature reached within a range of temperatures. For example, Snead et al. (ORNL) ${ }^{22}$ successfully used changes in resistivity to detect peak irradiation temperature with accuracies of approximately $20^{\circ} \mathrm{C}$ for dose ranges of 1 to $8 \mathrm{dpa}$ and temperatures between 200 and $800{ }^{\circ} \mathrm{C}$. Experimental data ${ }^{22}$ suggest that upper and lower bounds for this range may be extended.

The technique implemented by Snead et al.,22 is now also available at INL. Specialized equipment at INL's High Temperature Test Laboratory (HTTL) now allows peak temperature detection of static capsules using $\mathrm{SiC}$ monitors. In this technique, the $\mathrm{SiC}$ sensor electrical resistivity is measured after heating in a furnace located within a stainless steel enclosure at the HTTL (see Figure 26). After heating, cooled samples are placed into a constant temperature environmental test chamber to insure electrical resistivity measurements are taken within $0.2{ }^{\circ} \mathrm{C}$ of a predetermined temperature, $30^{\circ} \mathrm{C}$. A high accuracy ( 9 digit) multimeter, which is placed outside the stainless steel enclosure, is used to obtain resistance measure- 
ments. Specialized fixturing was developed to insure that measurements are always taken with the SiC sensors placed in the same orientation. A four point probe technique is used with the four points connected to the sample through spring-loaded angled electrodes that hold the $\mathrm{SiC}$ temperature monitor in place. Current and voltage are provided to the sample through wires that are threaded through the holes in the electrodes. The accuracy of this new INL capability was verified by completing tests with unirradiated samples of various grades of $\mathrm{SiC}$ temperature monitors (which vary in resistivity) and by completing comparison measurements with ORNL on identical sensors that had been subjected to identical irradiation conditions. ${ }^{23}$ Results, as shown in Figure 27, indicate that similar peak irradiation temperatures are inferred from ORNL and INL measurements for such cases.

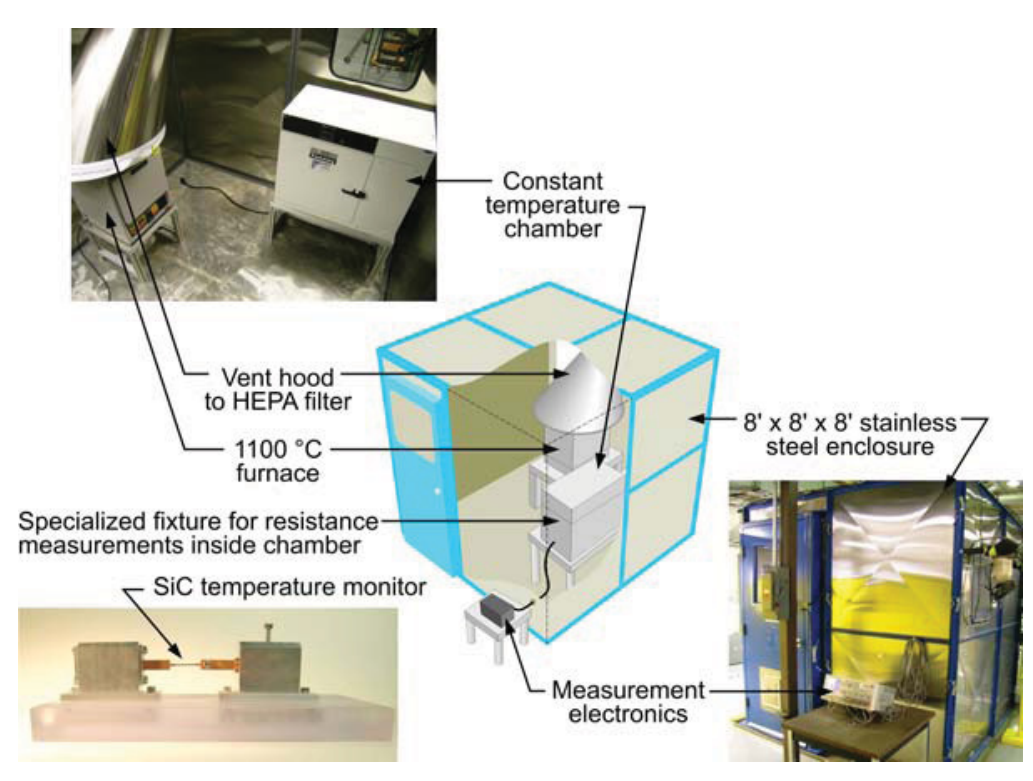

Figure 26. Setup to anneal and measure electrical resistivity of SiC temperature monitors.

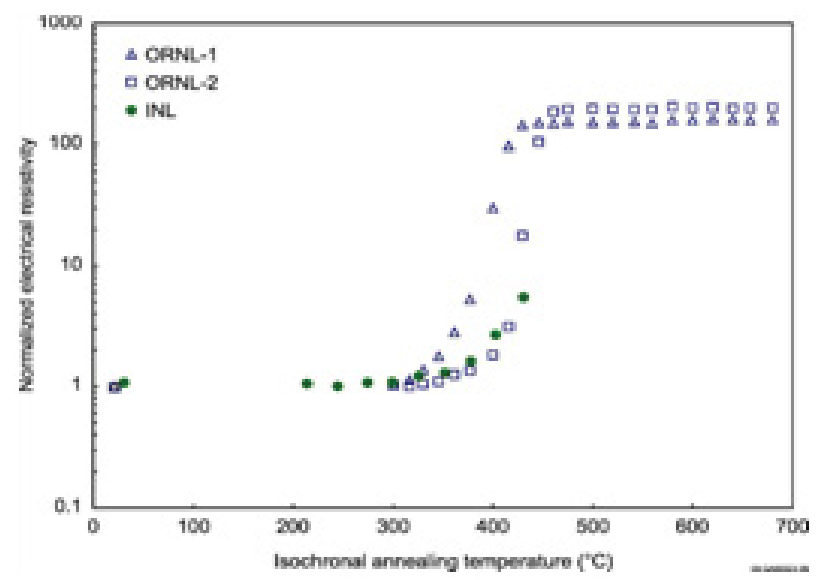

Figure 27. Electrical resistivity measurement comparison on $\mathrm{SiC}$ monitors irradiated at $300{ }^{\circ} \mathrm{C}$. 


\subsubsection{Instrumented Lead/Loop Tests}

In addition to sensors used to detect peak irradiation temperatures in static capsules, there are several sensors currently used to provide real-time temperature data. Capabilities of each of these sensors are described below.

Thermocouples. For decades, real-time temperature measurements during irradiation tests have been made with commercially-available, mineral insulated, metallic sheathed thermocouples. These thermocouples are used to monitor or/and control the temperature achieved during irradiation. For temperatures below $1000{ }^{\circ} \mathrm{C}$, experimental needs are typically met using Type $\mathrm{K}$ or Type $\mathrm{N}$ thermocouples, which have demonstrated excellent reliability and signal stability under irradiation, even for very high neutron fluences exceeding $10^{22} \mathrm{n} / \mathrm{cm}^{2}$ (thermal neutrons). However, these thermocouples decalibrate when exposed to temperatures above $1100{ }^{\circ} \mathrm{C}$. High temperature thermocouples, such as Type C or D thermocouples, decalibrate due to transmutation when their tungsten-rhenium thermoelements with high absorption cross sections are exposed to a thermal neutron flux. Hence, thermocouples were needed that can withstand both high temperature and high radiation environments.

To address this need, INL developed a High Temperature Irradiation Resistant ThermoCouple (HTIR-TC) sensor that contains commercially-available doped molybdenum paired with a niobium alloy. HTIR-TC component materials were selected based on data obtained from materials interaction tests, ductility investigations, and resolution evaluations (see References 24 through 28). HTIR-TC long duration performance has been demonstrated through testing, in which thermocouples were held at elevated temperatures (from $1200{ }^{\circ} \mathrm{C}$ to $1800{ }^{\circ} \mathrm{C}$ ) for up to 6 months. The $1200{ }^{\circ} \mathrm{C}$ test included nineteen commercially-available Type $\mathrm{N}$ thermocouples, three commercially-available Type K thermocouples, and nine INL-developed swaged HTIR-TCs. As indicated in Figure 28, some Type $\mathrm{K}$ and $\mathrm{N}$ thermocouples drifted by over $100{ }^{\circ} \mathrm{C}$ or $8 \%$. Much smaller drifts (typically less than $20^{\circ} \mathrm{C}$ or $2 \%$ ) were observed in the INL-developed HTIR-TCs. As documented in Reference 25, similar drifts (2\%) were observed in HTIR-TCs in a long duration (4000 hour) test completed at $1400{ }^{\circ} \mathrm{C}$.

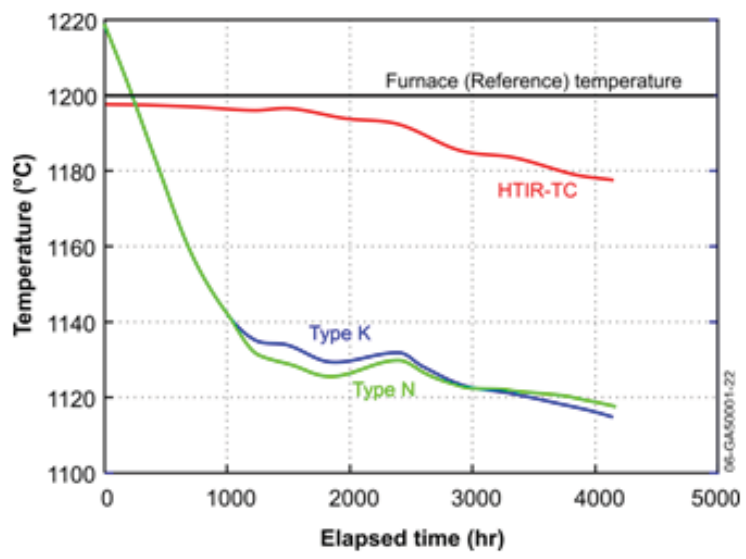

Figure 28. Representative thermocouple response in INL $1200{ }^{\circ} \mathrm{C}$ tests.

HTIR-TCs were also installed in a multi-capsule experiment where irradiated gas reactor fuel samples were irradiated at temperatures up to $1200{ }^{\circ} \mathrm{C}$ in INL's ATR. The test started in February 2007 and ended in October 2009. Figure 29 shows signals from two INL-developed HTIR-TCs and one Type N thermocouple located within one of the capsules (Capsule 4). Signal variations are due to ATR power fluctuations and outages (e.g., gray regions correspond to when the ATR was shutdown). As shown in this figure, the HTIR-TC (TC-4-1) located near the Type N thermocouple (TC-4-3) yielded a signal consistent with the signal from this Type $\mathrm{N}$ thermocouple at the beginning of this irradiation. In addition, the HTIR-TC 
located at a higher temperature region within the capsule (TC-4-2) yielded a consistent, but higher temperature, signal. However, in October 2008, the Type N thermocouple failed; and its signal ceased. The successful operation of HTIR-TCs in this test has led to INL supplying them to other test reactors around the world.
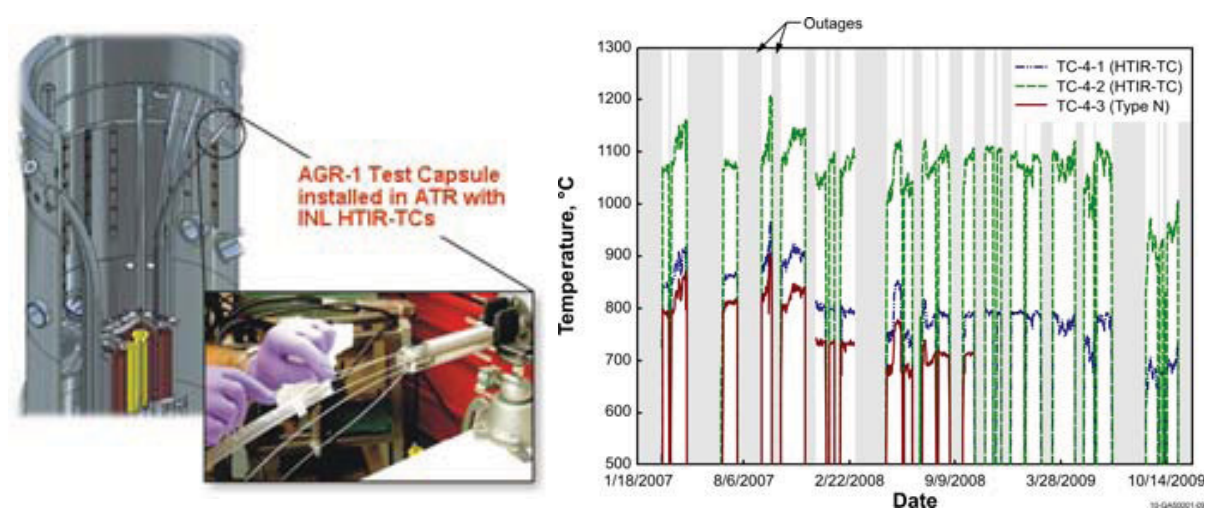

Figure 29. HTIR-TCs installed in AGR-1 test capsule and representative HTIR-TC and Type N data during ATR irradiation.

Expansion thermometers. The Institute for Energy Technology at the Halden Reactor Project (IFE/HRP) uses an expansion thermometer (ET) to monitor the average temperature during irradiation of nuclear fuel in the HBWR (see Figure 30). ${ }^{11}$ This sensor infers temperature using a Linear Variable Differential Transformer (see Section 4.5) to measure the thermal expansion of a tungsten rod that passes through a hole drilled through the center of a fuel stack. The ET is often used by IFE/HRP for long duration tests at high temperature. In long-term irradiations where transmutation is of concern, a low absorption cross section material, such as molybdenum, may be substituted for the tungsten rod.

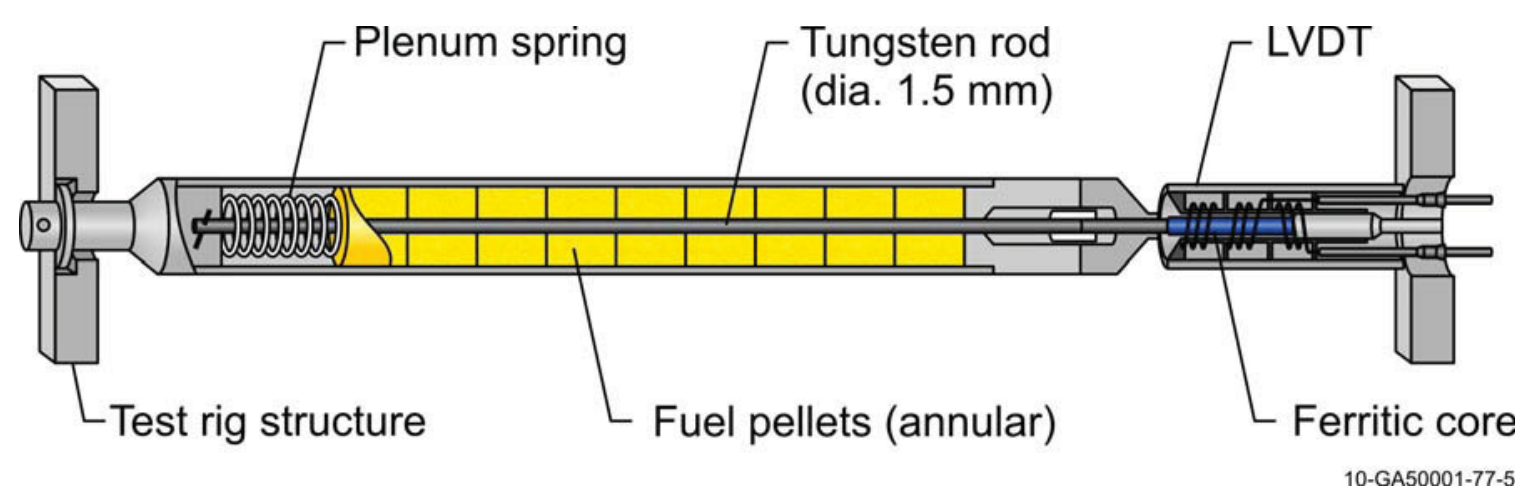

Figure 30. Schematic diagram of Expansion Thermometer (ET) used by IFE/HRP.

Ultrasonic thermometers. Ultrasonic thermometry has the potential to improve upon temperature sensors currently used for in-core fuel temperature measurements. Even though the HTIR-TCs developed by INL have overcome most of the difficulties associated with the nuclear-based application of thermocouples, the resistivity of electrical insulators can degrade if subjected to high temperatures $\left(>1800^{\circ} \mathrm{C}\right)$, causing shunting errors. Furthermore, thermocouples typically only allow measurement at a single location, and examination of melt wires or silicon carbide monitors only allow estimation of the maximum test temperatures at the point of installation. Ultrasonic thermometry offers the potential for real time in-pile measurement of a temperature profile using a single multi-segment sensor. Prior applications of ultrasonic thermometers 
have demonstrated the viability of this technology, but in-pile applications were primarily limited to high-temperature fuel damage tests, which ceased several decades ago.

INL recently initiated an effort to investigate the use of ultrasonic thermometry for measuring a temperature profile using a single, small diameter (typical diameters range from $0.25 \mathrm{~mm}$ to $1 \mathrm{~mm}$ ) multi-segment sensor. $^{28,29}$ Tests will be initiated at INL's HTTL to demonstrate the viability of and optimize ultrasonic techniques using the setup shown in Figure 31. Initial testing is focusing on a simplified, single segment probe. As part of this optimization effort, various design alternatives are being explored. Alternate wave guide, sensor, and sheath materials with high melting temperatures and insensitivity to neutron radiation (such as molybdenum), are under evaluation. Also, different methods of generating ultrasonic pulses (magnetostrictive and piezoelectric materials), as well as different pulse modes (longitudinal and torsional) are being considered. Ultimately, evaluations will focus on multiple segment probes, as a key feature of the ultrasonic thermometry system is the ability to measure a temperature profile. However, these sensors will also require development of a signal conditioning and processing system for the multiple signals generated by a multi-segment sensor. Ultimately, it is planned that the optimized UT design for in-pile temperature detection will be evaluated in an ATR NSUF test.

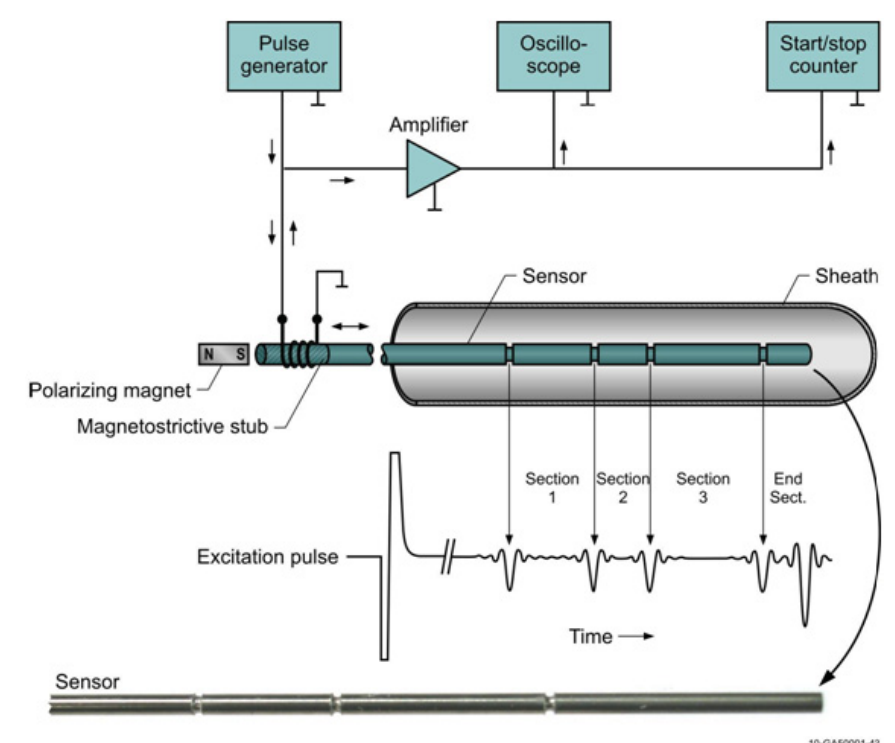

Figure 31. A typical multi-sensor pulse/echo ultrasonic thermometry system and 'notched' sensor.

\subsection{Thermal Conductivity}

Thermal conductivity is another key property that must be known during irradiation testing of fuels or structural materials. Thermal conductivity is highly dependent on the physical structure, chemical composition, and the state of the material. Currently, changes in fuel or material thermal conductivity during ATR irradiations are evaluated out-of-pile. However, as discussed in this section, real-time methods for detecting changes in thermal conductivity during irradiation in instrumented lead and loop tests are available.

\subsubsection{Static Capsule Tests}

There is no known method for real-time detection of thermal conductivity changes of materials irradiated in a static capsule. Integral changes in thermal conductivity can only be measured as part of post-irradiation examinations. 


\subsubsection{Instrumented Lead/Loop Tests}

Historically, in-pile thermal conductivity measurements have been made using an approach (see Figure 32a) with one (or more) thermocouples embedded near the center of the fuel rod and one exterior to the fuel (in the coolant or a structure outside the fuel element). As part of a collaborative effort with Utah State University (USU) and the Institute for Energy Technology at the Halden Reactor Project (IFE/HRP), INL is evaluating the multiple thermocouple steady-state thermal conductivity approach and a transient hot wire thermal conductivity method (with a single probe containing a line heat source and thermocouple embedded in the fuel as shown in Figure 32b) as candidate in-pile effective thermal conductivity measurement techniques. ${ }^{30-32}$ Evaluations compare the accuracy of each approach for various fuel types and test conditions. Details about the status of these evaluations are summarized in this section.
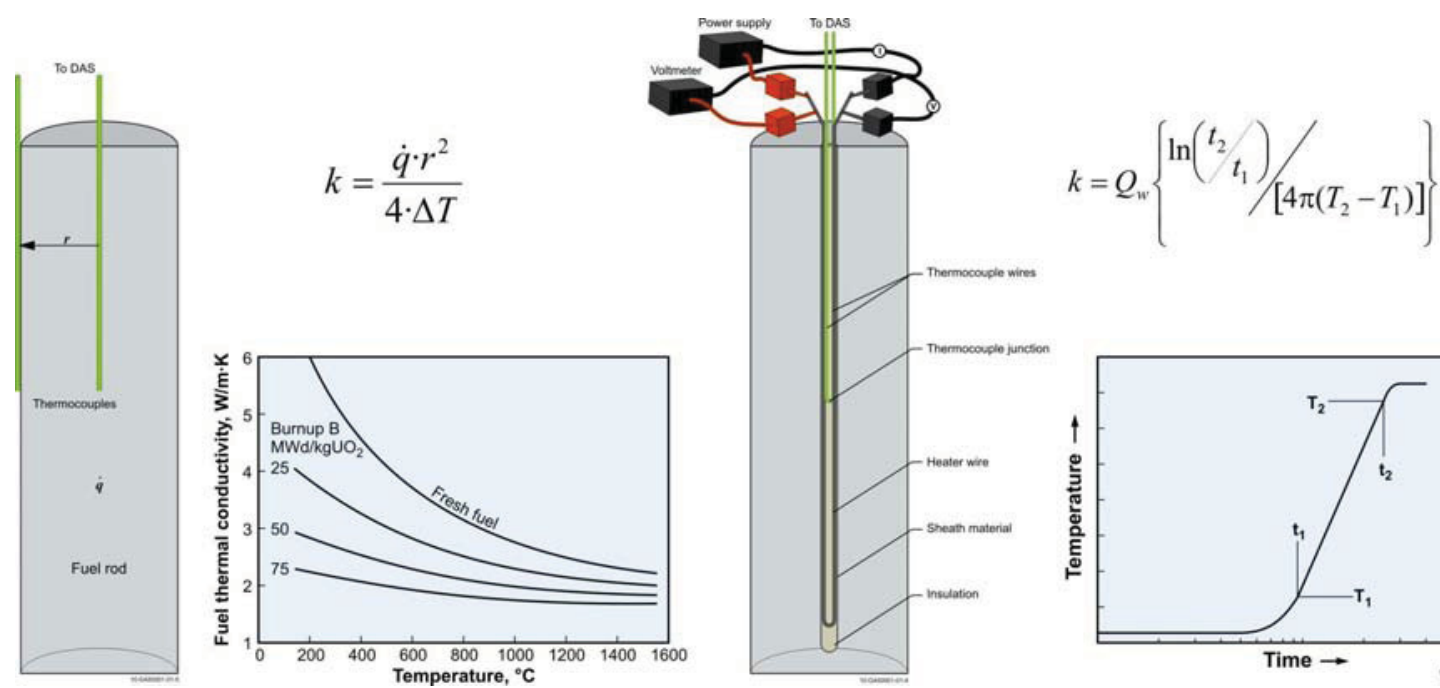

Figure 32. In-pile thermal conductivity methods under evaluation (see text for variable definitions).

Multiple thermocouple steady-state method. Variations of the two-thermocouple approach for detecting thermal conductivity changes in oxide and metal fuel during irradiation have been explored since 1960 (e.g., see References 33 through 35). Typically, results from this approach correlate in-pile degradation of fuel during irradiation (due to swelling, cracking, etc.), as an indication of thermal conductivity degradation (because the 'effective' thermal conductivity of the fuel, cladding, and gap are measured, and one is really just detecting all the changes that impact the thermal conductivity). Most earlier efforts were conducted with metal fuel pellets encased in cladding with sodium bonding (thus, minimizing any concerns about fuel pellet-to-gap resistance). The HBWR is currently the only test reactor where in-pile thermal fuel thermal conductivity measurements are performed using a multiple thermocouple approach.

INL and USU completed evaluations to estimate the thermal conductivity of a surrogate fuel rod using two thermocouples inserted into a surrogate fuel rod with volumetric heat generation simulated by Joule heating. One thermocouple monitors centerline temperature, while the other monitors temperature at a measured radial position within the rod. As shown in Figure 32a, the thermal conductivity, $k$, is calculated from the radial temperature drop, $\Delta \mathrm{T}$, the radial distance between the thermocouples, $r$, and volumetric heat generation, $\dot{q}$. High temperature carbon structural foam was selected as the surrogate material in these evaluations because of its electrical resistivity, low thermal conductivity, and high temperature resistance to oxidation and melting. Figure 33 shows a schematic of the surrogate rod and test setup used to obtain data. Surrogate rods containing two thermocouples (Figure 33a) were positioned inside a tube furnace with an argon cover gas to provide a controlled sample test temperature (Figure 33b). Voltage and current from the 
power supply were supplied to the rod using Inconel electrodes connected to Inconel clamps. Leads attached to Inconel clamps at each end of the rod were used to measure the voltage drop across the rod. A precision current shunt was used to measure current within the experimental test loop. Volumetric heat generation was calculated using the measured current, $I$; and the sample voltage drop, $V$; sample dimensions, and the fact that power is the product of the current and voltage. Signals were processed by a data acquisition system to give temperatures from thermocouples and power in the sample. Results for tests with the supplied power held constant at $100 \mathrm{~W}$ in the 500 to $700{ }^{\circ} \mathrm{C}$ temperature range were within $2 \%$ $8 \%$ of the surrogate rod thermal conductivity values obtained using material property measurement systems (e.g., laser flash diffusivity, pushrod dilatometry, and differential scanning calorimetry) available at INL's HTTL.

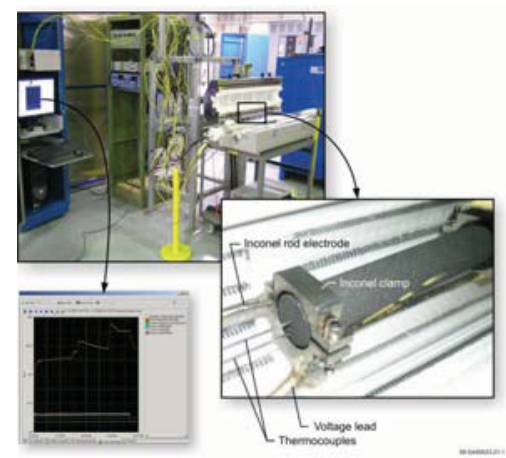

(a)

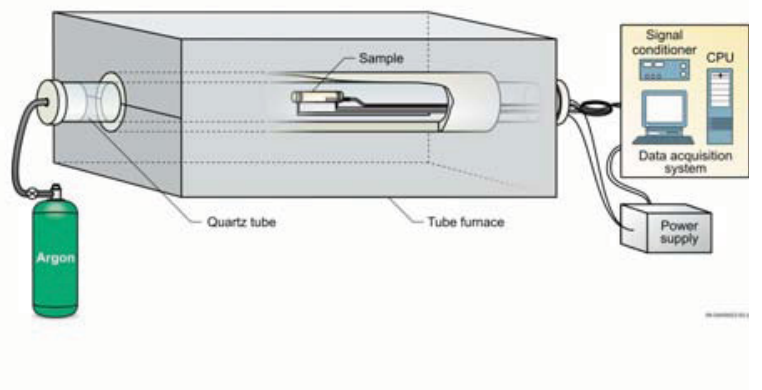

(b)

Figure 33. Setup for evaluating the two thermocouple method.

Hot-wire Transient Needle Probe. USU/INL also evaluated the viability of using a needle probe based on the Transient Hot Wire Methods (THWMs) or the line heat source method that was first suggested by Schleirmacher. ${ }^{36}$ Numerous references may be found in the literature describing applications of this method to measure the thermal conductivity of solids, fluids, and gases (e.g., see References 37 through 42). The THWM is applied by embedding a line heat source in a sample whose thermal conductivity is to be measured. From a condition of equilibrium, the heat source is energized and heats the sample with constant power. The temperature response of the sample is a function of its thermal properties. As indicated in Figure $32 \mathrm{~b}$, the thermal conductivity, $k$, is calculated from a relation that includes $Q_{w}$, the linear power dissipated by the heater and the measured increase in temperature, $T_{2}$ and $T_{1}$, between times, $t_{2}$ and $t_{1}$.

INL/USU also are exploring the use of a hot wire probe design, containing a resistance heater and thermocouple embedded in a single probe (see Figure 34), that was developed based on ASTM D $5334-05.18 .^{42}$ As shown in Figure 34b, the probe contains a heat source element and a temperature sensor inserted into a sample. The thermal conductivity is determined from the temperature rise in the sample. Needle probes were designed and fabricated at INL's HTTL for both room temperature proof-of-concept evaluations and high temperature testing. Using the setup shown in Figure 34, experimental results show that the INL/USU designed needle probes can measure the thermal conductivity of fused silica, the ASTM recommended reference material, within $2 \%$ at room temperature, $250{ }^{\circ} \mathrm{C}, 400{ }^{\circ} \mathrm{C}$, and $600{ }^{\circ} \mathrm{C}$. In these evaluations, the probe design was selected such that materials and geometry were optimized to improve accuracy for the proposed test temperature and surrogate rod material (e.g., wire diameters and materials used for probes differed to optimize heating in the surrogate fuel rod and reduce other losses). These optimization techniques are being implemented for an upcoming irradiation test in the Massachusetts Institute of Technology Nuclear Research Reactor (MITR), where the distance from the fuel rod to the data acquisition equipment is even longer with more dramatic temperature differences. 


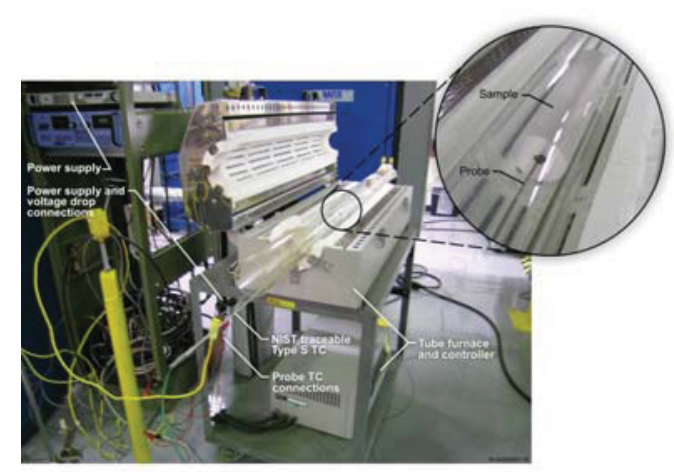

(a)

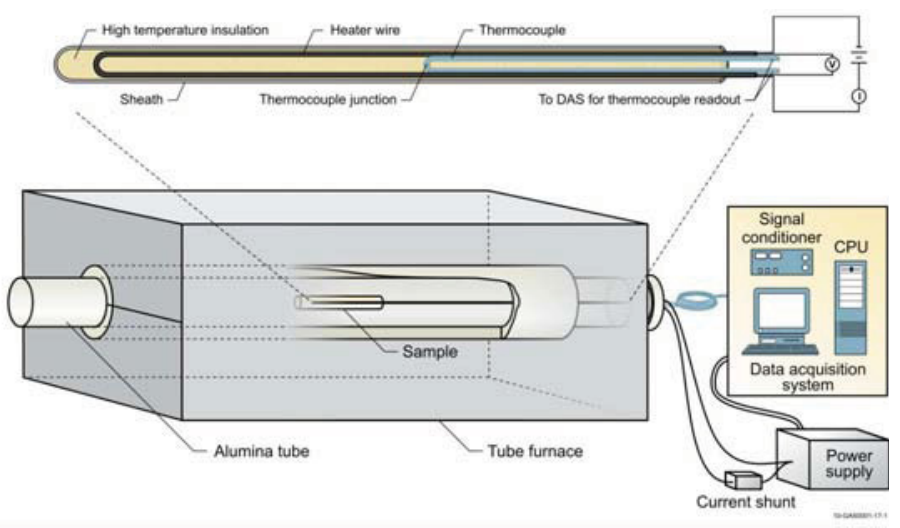

(b)

Figure 34. Setup for evaluating transient hot wire needle probe.

Evaluations are continuing to gain insights about the limitations and benefits of each method and its viability for in-pile applications. Initial results suggest that the transient method reduces the impact on the sample because it only requires a single small centerline sensor. Furthermore, the transient method requires shorter measurement times; and it eliminates the need to quantify 'uncertain' parameters, such as fuel-to-cladding contact resistance or coolant convective heat transfer coefficients, that are required for two-thermocouple approaches. However, additional evaluations are needed to gain insights about the limitations of this approach with respect to substrate thermal conductivity, material size, and long term reliability of these needle probes.

\subsection{Specific Heat Capacity}

\subsubsection{Static Capsule Tests}

There is no known method for real-time detection of specific heat capacity changes of materials irradiated in a static capsule. Integral changes can be inferred only from post-irradiation examinations.

\subsubsection{Instrumented Lead/Loop Tests}

There is no known method for real-time detection of specific heat capacity changes of materials irradiated in instrumented lead or PWR loop tests. Detection of in-pile specific heat changes might be possible using comparative techniques with a sample having a well-characterized temperature-dependent specific heat capacity, similar to the approach used in differential scanning calorimetry systems. However, no known research and development effort is currently exploring such an option.

\subsection{Density/Thermal Expansion}

\subsubsection{Static Capsule Tests}

There is no known method for real-time detection of density or thermal expansion changes of materials irradiated in a static capsule. Integral release can be inferred only from post-irradiation examinations.

\subsubsection{Instrumented Lead/Loop Tests}

There is no 'direct' method for real-time detection of density changes of materials irradiated in an instrumented lead or PWR loop tests. However, density changes can be inferred by combining detected changes in geometry (e.g., length and diameter) using techniques described in Section 4.5. 


\subsection{Elongation/Deformation/Creep/Swelling}

Geometry changes of fuel irradiated in the ATR are currently evaluated out-of-pile after specified lengths of irradiation time. However, efforts are underway to evaluate several options that offer the potential to obtain real-time length and diameter data from samples irradiated in the ATR. For lower temperature (up to $500{ }^{\circ} \mathrm{C}$ ) applications, commercially-available Linear Variable Differential Transformers (LVDTs) are being evaluated as a near-term option for detecting geometry changes in ATR irradiations. In addition, as discussed in Section 5.2, ultrasonic transducers are being explored as a more compact sensor that can measure geometry changes and crack growth with higher precision in multiple dimensions at higher temperatures.

\subsubsection{Static Capsule Tests}

There is no known method for real-time detection of elongation changes of materials irradiated in a static capsule. Integral changes can be detected only from post-irradiation examinations.

\subsubsection{Instrumented Lead/Loop Tests}

A review of the literature indicates that most test reactors currently rely on LVDTs to detect changes in length during irradiation (although strain gauges have been explored in earlier tests).

Strain Gauges. Earlier references ${ }^{43,44}$ report using miniature strain gauges encased in zircaloy sheaths welded on the cladding in the circumferential and axial directions. These references indicate that strain gauge measurements were unstable due to high sensitivities of the gauges to temperature and neutron fluence.

LVDTs. LVDTs are electrical transformers with three coils placed end-to-end around a tube (see Figure 35a). The center coil is the primary, and the two outer coils are the secondaries. A cylindrical magnetically-permeable core, attached to the object whose position is to be measured, slides along the axis of the tube. An alternating current is driven through the primary, causing a voltage to be induced in each secondary which is proportional to its mutual inductance in the primary. As the core moves, these mutual inductances change, causing the voltages induced in the secondaries to change. The coils are connected in reverse series, so that the output voltage is the difference between the two secondary voltages. When the core is in its central position, equidistant between the two secondaries, equal but opposite voltages are induced in these two coils, so the output voltage is zero (see Figure 35b). Many features of LVDTs (e.g., frictionless measurements, long lifetime, high resolution, etc.) make them ideal for in-pile applications.
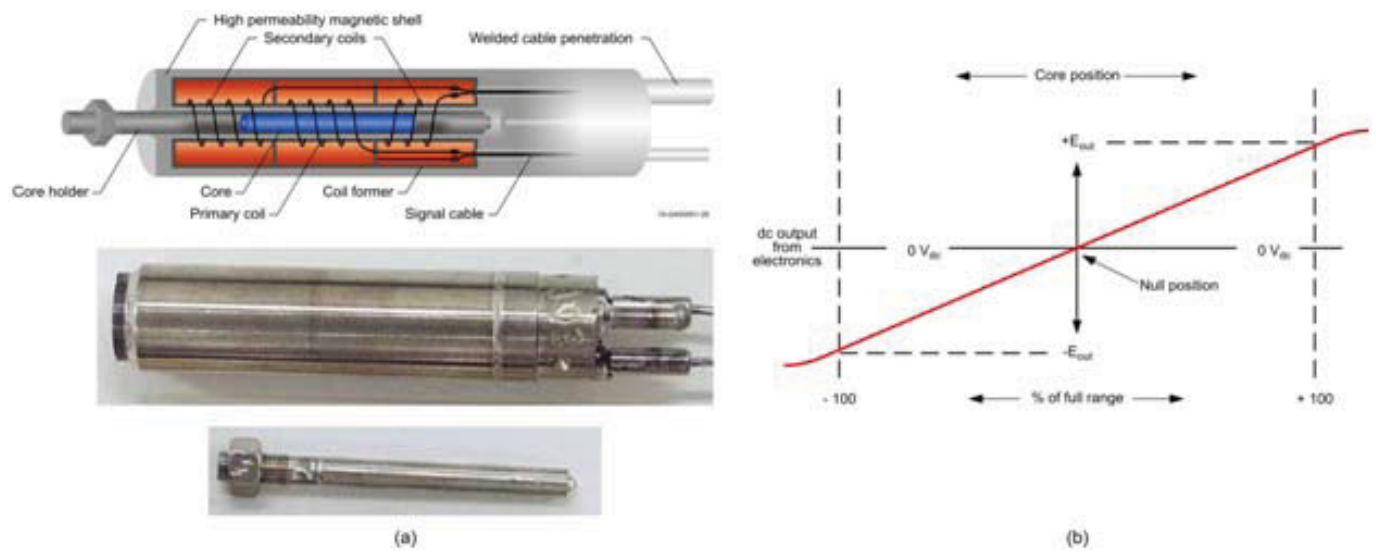

Figure 35. LVDT components (a) and operation (b).

Most research reactors rely on LVDTs made by the IFE/HRP. In the IFE/HRP LVDTs, the primary coil is activated by a constant current generator (at $400-2500 \mathrm{~Hz}$ ). The position of the magnetically-permeable 
core can be measured with an accuracy of $\pm 1-10 \mu \mathrm{m}$ (references vary on this value). Since the HRP started with in-core measurements, more than 2200 LVDTs of different types have been installed in different test rigs in the HBWR and other test reactors around the world. A failure rate of less than $10 \%$ after 5 year of operation is expected for their LVDTs operating in BWR, PWR or CANDU conditions. Recently, IFE/ HRP explored several improvements for LVDTs. For example, the IFE/HRP is collaborating with INL to investigate the use of alternate coil wires and design modifications to improve the performance of LVDTs at higher temperatures.

LVDT-based Cladding and Fuel Elongation (length). The IFE/HRP uses LVDTs to monitor cladding and fuel elongation as shown in Figure 36. ${ }^{11}$ For cladding elongation, the magnetically-permeable core is fixed to the fuel rod end plug, which is welded to the cladding. The upper part of the fuel rod is fixed to the rig structure (in a grid plate). The lower end is free to move, and the core is inserted into an LVDT which is fixed to another grid plate in the rig structure. Changes in cladding length are detected by the LVDT, thus providing cladding axial deformation data. For fuel elongation, the magnetic core is attached to a test rod, which is attached to a spring-loaded plate on top of the fuel stack. The core assembly is held firmly against the fuel stack by the spring. Fuel (axial) swelling/densification is then detected by the LVDT.

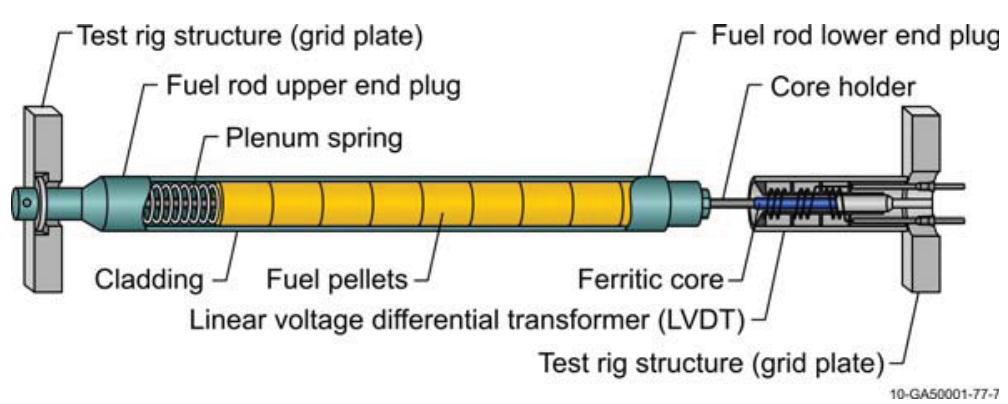

(a)

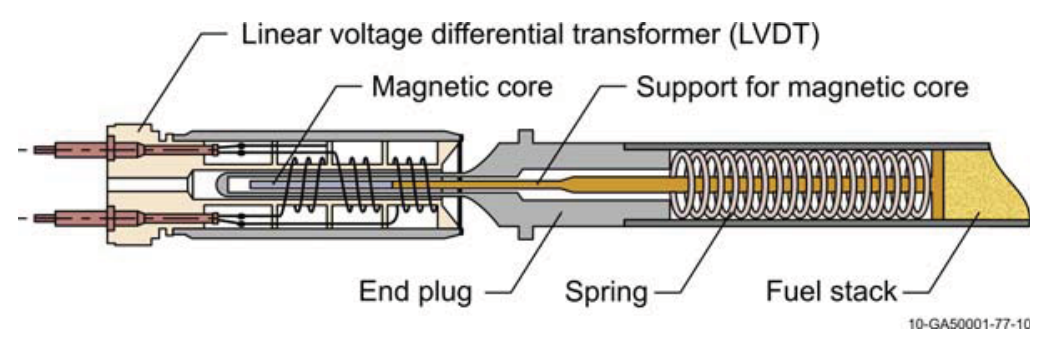

(b)

Figure 36. IFE/HRP test setups for detecting (a) cladding elongation; and (b) fuel elongation.

LVDT-based Diameter Gauges (diameter). Specialized IFE/HRP sensors have been developed that allow on-line monitoring of various parameters, such as fuel diameter. The IFE/HRP diameter gauge enables on-line measurement of cladding diameter for assessing cladding creep, pellet-cladding mechanical interaction, fuel creep / relaxation and fuel rod crud deposits. The representative test rig shown in Figure 37a provides real-time in-pile detection of fuel diameter changes, temperature, neutron exposure and loop coolant temperature, flow, and chemistry (e.g., concentration, $\mathrm{pH}$, etc.). IFE/HRP relies on LVDT-based technology in this diameter gauge. As shown in Figure 37b, the diameter gauge contains a differential transformer with two feelers on opposite sides of the fuel rod. During testing, the diameter gauge travels along the fuel rod using an in-core hydraulic drive and positioning system. The accuracy of the diameter gauge is $\pm 2 \mu \mathrm{m}$, and a calibration is performed in conjunction with each diameter trace by having calibra- 
tion steps on both fuel rod end plugs. The diameter gauge can operate at up to 165 bar and $325{ }^{\circ} \mathrm{C}$, However, IFE/HRP is investigating the potential to increase this temperature limit and sensor accuracy using alternate components and fabrication processes.

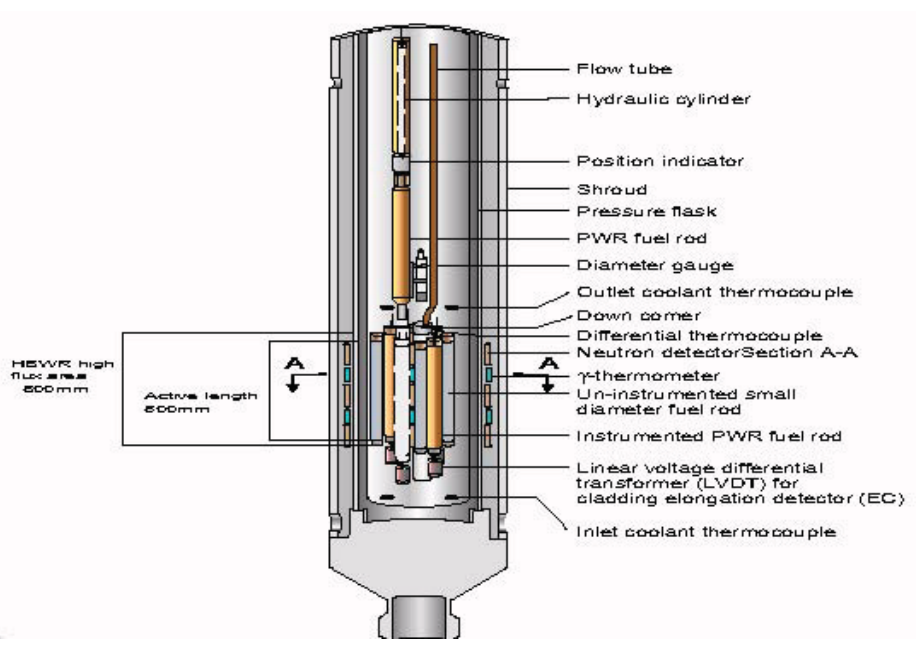

(a)

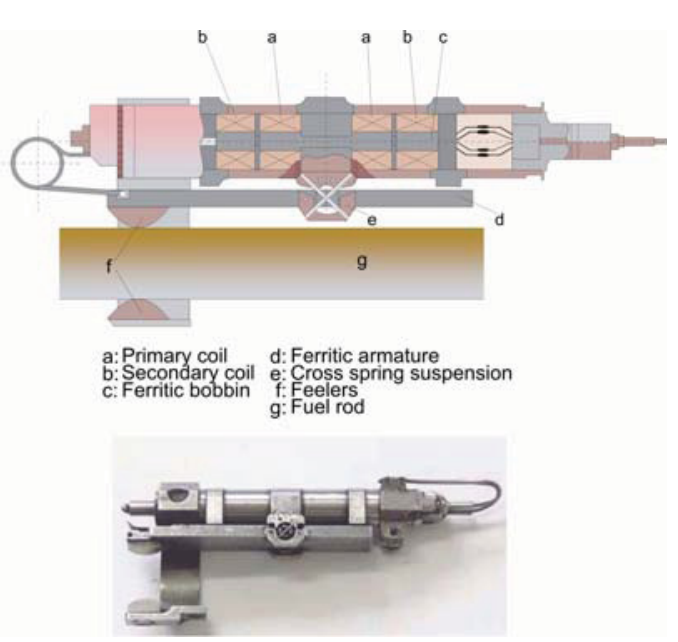

(b)

Figure 37. HRP fuel pellet cladding interaction/crud deposition test rig (a) with diameter gauge (b).

LVDT/bellows-based Creep and Tensile Test Rigs. Several organizations (e.g., VTT Technical Research Center of Finland, INL, KAERI, and CEA) have been involved in developing and deploying in-pile tensile test rigs to detect growth of tensile and creep specimens using a bellows to apply a load to a specimen and LVDTs to detect growth of the specimen. Although it is unlikely that the primary damage experienced by a specimen will be affected by the applied stress during irradiation, the subsequent process of dislocation formation, that is responsible for radiation hardening, yield drop, and plastic flow localization, will be substantially altered by the applied stress. Furthermore, it is speculated that the fatigue lifetime during in-situ cyclic loading experiments may be significantly different from the ones obtained during fatigue experiments on specimens in the post-irradiated condition. Hence, several organizations are pursuing an in-situ material creep testing system for inclusion in PWR loops. Figure 38 shows the system developed by VTT and tested in the BR-2 reactor. This system includes a pneumatic loading unit, which loads a test specimen using gas to pressurize metallic bellows, and a LVDT from IFE/HRP to measure the resulting displacement produced in the tensile specimen. The outside diameter of the module tested in the BR-2 reactor is $25 \mathrm{~mm}$, and the total length of the module with the LVDT is $150 \mathrm{~mm}$.

INL is currently developing an in-pile creep test rig for deployment in an ATR PWR loop. As discussed in References 45 and 46, a prototype of the prototype creep test rig shown in Figure 39 was recently evaluated in an autoclave at INL's HTTL. Initial evaluations with stainless steel tensile specimens in the elastic region yield data that are consistent with results obtained from a load frame for this material. Testing in the plastic region has also shown very close agreement between LVDT measurements and post-test micrometer measurements for stainless steel and copper tensile specimen. It is planned to initiate irradiation testing in an ATR PWR loop in 2011. 

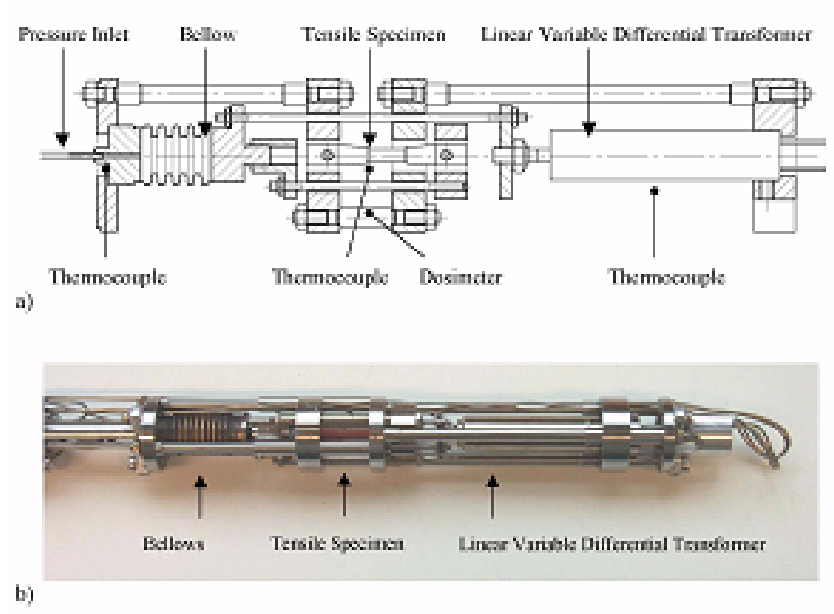

c)

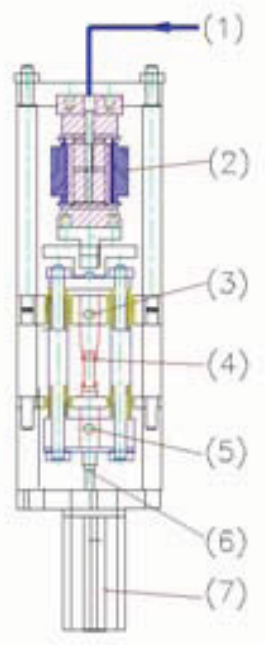

Figure 38. Tensile test module irradiated in the BR-2 ( $a$ and $b$ ) with schematic of tensile test module (c) that includes: (1) gas line, (2) pneumatic loading unit, (3) firm specimen fixing point, (4) specimen, (5) movable specimen fixing point, (6) LVDT plunger and (7) LVDT holder.
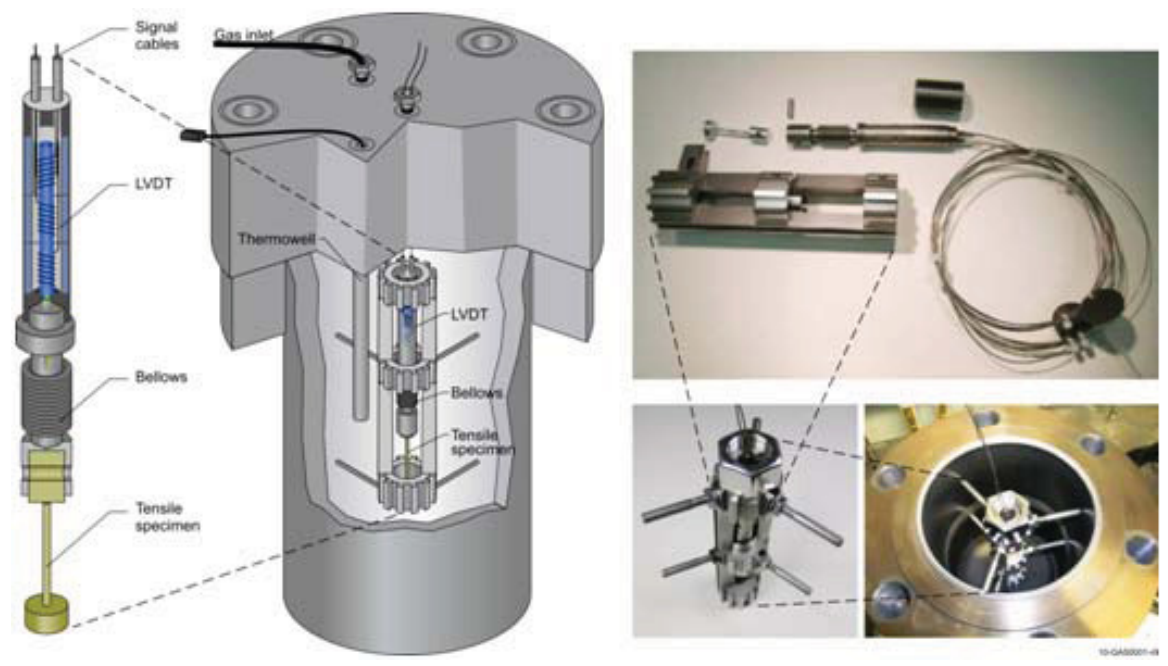

Figure 39. Creep test rig positioned in autoclave for testing.

\subsection{Cracking/Crack Initiation and Growth}

\subsubsection{Static Capsule Tests}

There is no known method for real-time detection of crack initiation or growth in materials irradiated in a static capsule. Integral changes can be detected only from post-irradiation examinations.

\subsubsection{Instrumented Lead/Loop Tests}

Direct Current Potential Drop. Crack initiation and growth of samples irradiated in instrumented lead and PWR loop tests in the ATR are detected out-of-pile. However, crack-growth rates in core structural component materials irradiated in the HBWR are monitored in-pile using miniaturized Compact Tension (CT) specimens and the "direct current potential drop method." The direct current potential drop method (see Figure 40) is based on sending an electrical current through the specimen and measuring the potential 
(voltage) at several locations on the CT-specimen. The measured potentials depend on the propagation of the crack. Thus, the crack-length can be determined from the measured potential drops. IFE/HRP has applied this technology to detect the impact of coolant parameters ( $\mathrm{pH}$, impurities, boron concentration, etc.) on fuel cladding corrosion. In addition, IFE/HRP can perform such measurements on pre-irradiated fuel removed from commercial reactors. As part of an ATR NSUF-funded effort, MIT developed a crack growth test rig design that could be deployed in the ATR. Depending on the availability of future funding, a prototype of this test rig will be built and tested in an autoclave at the HTTL.

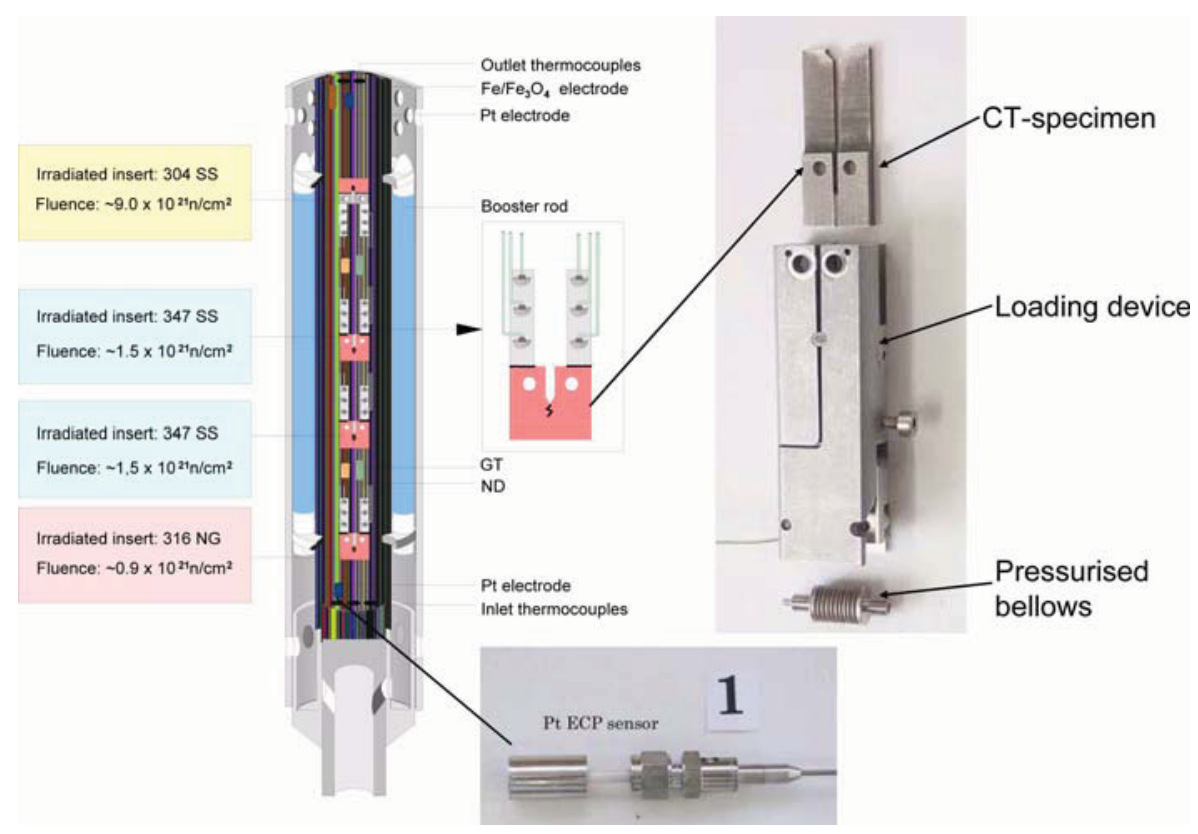

Figure 40. Crack growth test rig.

\subsection{Young's Modulus}

\subsubsection{Static Capsule Tests}

There is no known method for real-time detection of Young's Modulus of materials irradiated in a static capsule. However, measurements can be obtained during post-irradiation examinations

\subsubsection{Instrumented Lead/Loop Tests}

Currently, any measurements of Young's Modulus of materials irradiated in ATR instrumented lead tests or PWR loops are completed during post-irradiation examinations. However, the creep or tensile test rigs, such as the one deployed by VTT in the BR-2 with load control (see Section 4.5.2), could be used for in-pile measurement of Young's Modulus.

\subsection{Fission Gas Release/Rod Internal Pressure}

\subsubsection{Static Capsule Tests}

There is no known method for real-time detection of fission gas release from a static capsule. Integral release can be inferred only from post-irradiation examinations.

\subsubsection{Instrumented Lead/Loop Tests}

As indicated in this section, several approaches are available for detecting fission gas release or rod internal pressure. 
Rod Internal Pressure (LVDT/bellows-based). IFE/HRP monitors fuel rod internal pressure to gain insights about fission gas release during irradiation. ${ }^{11}$ The pressure transducer consists of a miniaturized bellows mounted in the fuel rod end plug. As indicated in Figure 41, a magnetic core is fixed to the free moving end of the bellows; the other end of the bellows assembly is fixed to the end plug. The bellows is pressurized to typically 2 bar less than the initial rod pressure and seal welded. Bellows/core movements are sensed by an LVDT. The IFE/HRP pressure transducers are available for different pressure ranges. The most common ranges have a $\Delta \mathrm{p}$ (internal versus external pressure of the bellows) of 15 bar (220 psi) to 70 bar (1020 psi). Typically, the uncertainty of these pressure transducers is estimated to be from \pm 0.2 bar (2.9 psi) to \pm 0.5 bar (7.3 psi).

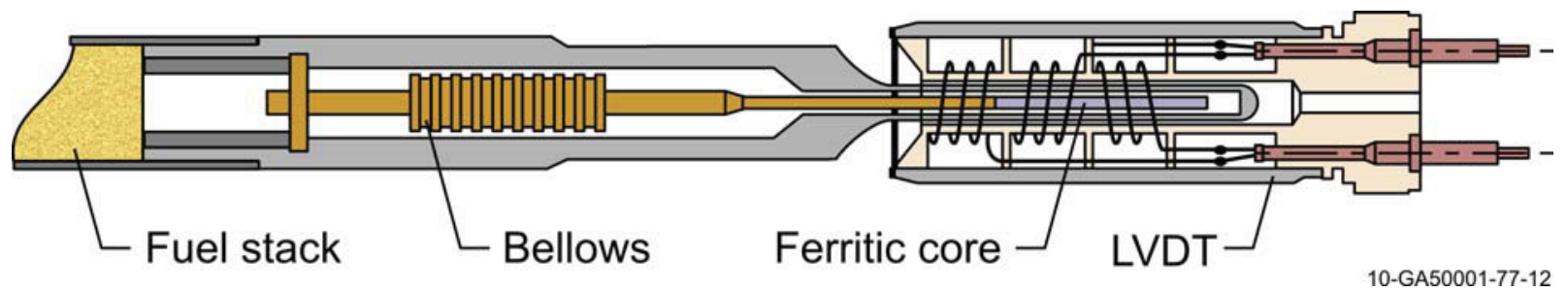

Figure 41. IFE/HRP fuel pressure gauge.

Rod Internal Pressure (bellows-based). CEA monitors fuel rod internal pressure using sensors that detect the balance between the pressure inside the fuel rod and a counter-pressure monitored from outside the fuel. ${ }^{6}$ It consists of two gas cavities, separated by metallic bellows as shown in Figure 42 . The first cavity communicates with the internal fuel rod pressure. The second cavity is connected to an external helium circuit, which is called the "counter-pressure" circuit. The imbalance between the internal rod pressure and the counter-pressure is accurately detected by two electric contacts, activated by the motion of the bellows. This sensor has been qualified in OSIRIS irradiations, and it has been successfully placed on pre-irradiated PWR fuel rods. The results demonstrate accuracies of \pm 0.32 bar on the pressure range tested (up to 120 bars).
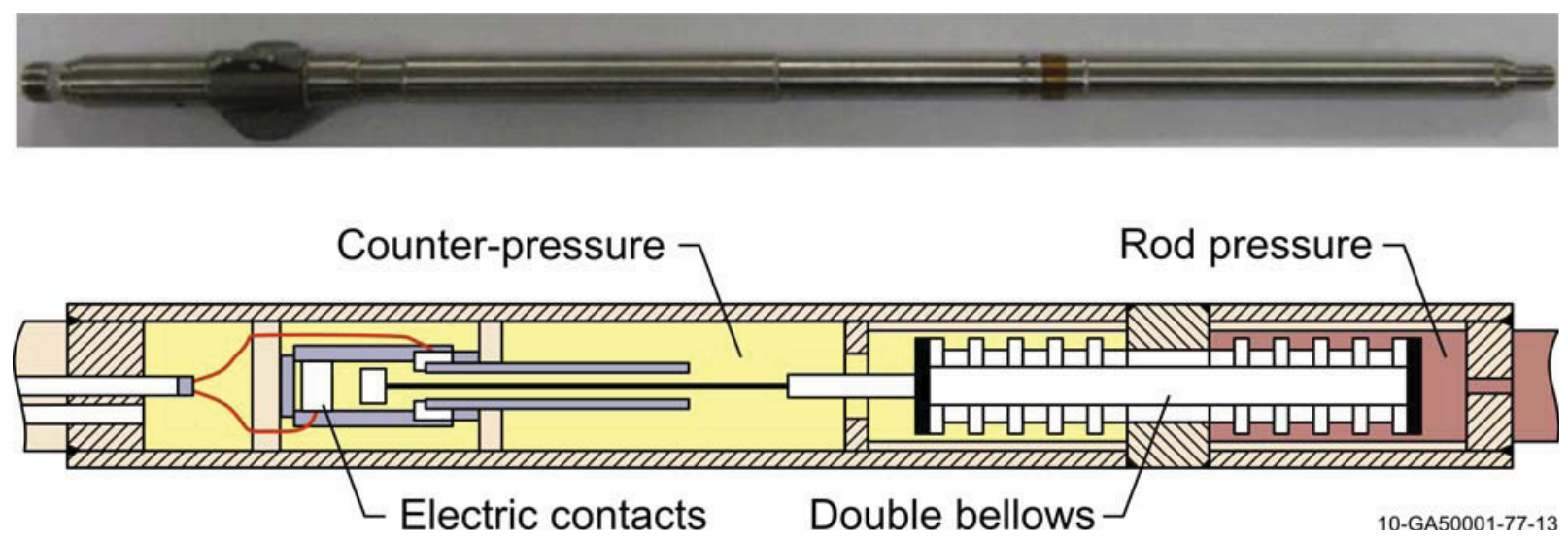

Figure 42. CEA counter-pressure sensor for measuring fission gas release.

Composition and Production Rate (sampling). Fission gas production can be detected by flowing an inert gas through channels containing samples, with and without cladding, and measuring the gamma rays emit- 
ted directly from the gas or from a trap outside the channel. By using a small, but known, amount of a radioactive gas in the flowing system, relative measurements can be turned into absolute measurements as a function of fuel mass, flux, temperature, aspect ratio (mass to surface area), and manufacturing processes; and thus, these measurements provide information about fuel response and fission gas production.

This type of system was successfully used in the AGR-1 gas reactor fuel experiment as a monitor for fuel cladding failure. As discussed in Reference 47, the Fission Product Monitoring System (FPMS) used for the AGR-1 fuel provided near real-time data indicative of the test fuel performance. The FPMS incorporated both high-purity germanium (HPGe) gamma-ray spectrometers and sodium iodide [NaI(Tl)] scintillation detector-based gross radiation monitors. To quantify the fuel performance, release-to-birth ratios (R/ B's) of radioactive fission gases were computed. The gamma-ray spectra acquired by the AGR-1 FPMS were used to determine the released activities of specific fission gases, while a dedicated detector provided near-real time count rate information. Isotopic build up and depletion calculations provided the associated isotopic birth rates. For the AGR-1 test, the outlet gas from each capsule was routed to individual fission product monitors as shown in Figure 43. However, capsule outlet flows could also be rerouted to an online spare monitor if any monitors experience detector or other failures. There was also the capability to take a grab sample of the effluent gas from each capsule. The fission product monitors consisted of a spectrometer for identifying and quantifying the fission gas nuclides and a gross gamma detector to detect when a puff release of fission gases passed through the monitor (such a puff would typically indicate when a fuel failure might have occurred). The gross gamma detector could also indicate release timing. With the combination of a gross gamma detector and a spectrometer being continuously online, the gross gamma detector results were scanned quickly to establish which portions of the voluminous spectrometer data needed to be closely scrutinized.
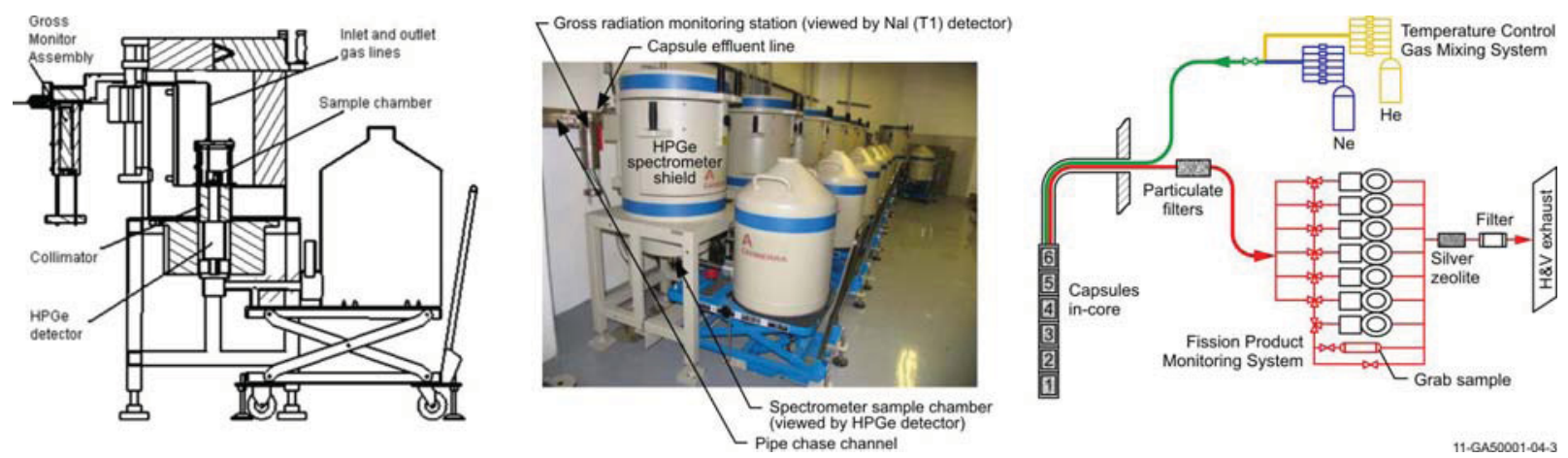

Figure 43. Setup for monitoring gas release during irradiation testing during the AGR-1 experiment flow path at the ATR.

Fission gas composition and pressure by an acoustic sensor. A dedicated acoustic sensor (containing a piezoelectric transducer) has been developed by CEA to measure online fission gas release in a fuel rod during irradiation experiments. Figure 44(a) illustrates some of the details related to the design of this acoustic fission gas release sensor. ${ }^{6}$ This assembly is composed of a small cylindrical cavity containing the gas to be analyzed. The upper part of the cavity is closed by a thin stainless steel plate. The piezoelectric transducer is fixed on this plate, in order to generate and measure acoustic waves through the plate in the gas cavity. Wires are directly welded on the piezoceramics electrodes. Acoustic waves propagate in the gas inside the cavity which is connected to the fuel rod plenum. The measurement of the reflected waves allows determination of the acoustic impedance of this system. Figure 17(b) illustrates the acoustic sensor principle. The signal and its echoes are recorded, and the time of flight of the signal and its attenuation are measured. From these measurements, it is possible to deduce simultaneously the molar mass of the gas 
(from the acoustic waves velocity) and the pressure of the gas (from the echoes attenuation). The online assessment of these two parameters is then used to obtain information regarding the fraction of fission gases released in the fuel rod.
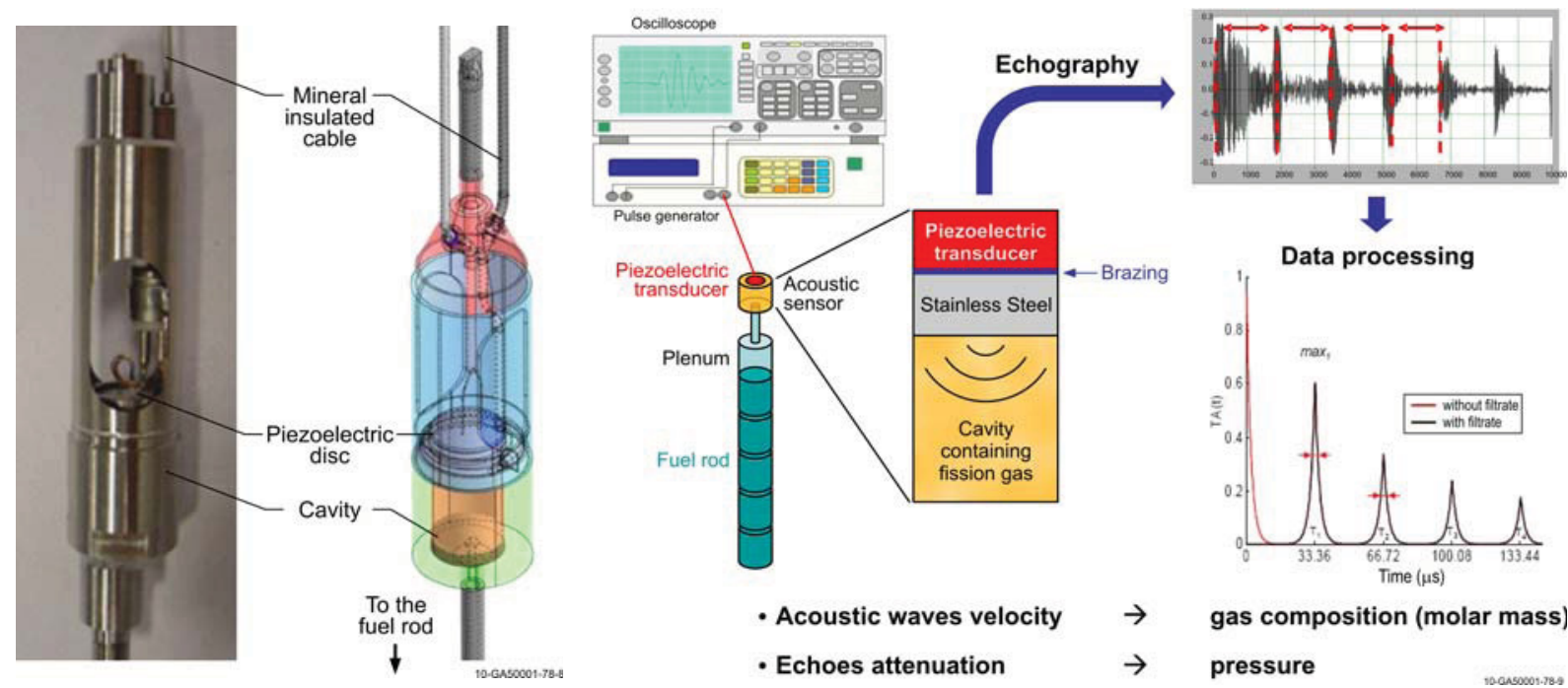

Figure 44. CEA fission gas release pressure and composition detection (a) sensor and (b) system operation.

Initial laboratory testing of acoustic sensor prototypes have been completed by CEA. In 2008, final sensors for use on a pre-irradiated fuel rod were designed and manufactured. The irradiation experiment REMORA-3, which started in 2010 in OSIRIS, includes this sensor installed on a pre-irradiated Pressurized Water Reactor fuel rod.

\subsection{Flux and Fluence}

Typically, ATR and HFIR tests use flux wires and post-irradiation evaluations to detect the total fluence to which the fuel or sample was exposed. However, MTRs in Norway, France, the Netherlands, Japan, Belgium, and the Republic of Korea routinely incorporate self-powered neutron detectors (SPNDs) during irradiation tests to gain real-time knowledge of the fission reaction rate. In recent years, French and Belgium test reactors have also incorporated specialized fission chambers to provide users real-time data related to neutron flux. Various flux detection methods are described in this section. During FY10, a joint ISU/CEA/INL effort was initiated to explore the use of fission chambers and SPNDs at the ATRC and ultimately, the ATR. As discussed in Section 3.1.3, specialized fixturing has been developed and installed at the ATRC for evaluating real-time flux sensors.

\subsubsection{Static Capsule Tests}

Flux Wires/Dosimeter Foils. The neutron flux profile and/or fluence in static capsule, instrumented tests, and PWR loop tests can be evaluated from flux wires placed around specimens in experimental devices. In the U.S., flux wire measurements are typically performed in accordance with ASTM E481-03. ${ }^{48}$ Flux wire materials are selected so that the desired energy level of the neutrons can be detected. Flux wires placed in a neutron flux activate and emit delayed gamma rays. When the material is removed from the reactor, its gamma emissions are measured on a germanium detector; and the neutron flux profile and fluence within an experiment can be evaluated from that data. 


\subsubsection{Instrumented Lead/Loop Tests}

Although ATR and HFIR irradiations do not typically include real-time methods for directly detecting thermal neutron flux and fission reaction rates for irradiation capsules, it is possible to directly measure these parameters without resorting to complicated correction factors. In addition, it is possible to directly measure fission reaction rates and to provide time-dependent monitoring of the fission reaction rate or fast/ thermal flux during transient testing.

Self-Powered Neutron Detectors (SPNDs). SPNDs typically consist of a neutron sensitive emitter material (e.g., vanadium, cobalt, rhodium, gadolinium, or hafnium) encased in a sheath (see Figure 45). Typical SPNDs are 1.4 to $1.6 \mathrm{~mm}$ in diameter. In the design shown in Figure 45, the emitter has a cylindrical shape and is positioned $\sim 12.7 \mathrm{~mm}$ from one end of the tube, which is seal welded. The emitter material is insulated from the tube sheath using compacted aluminum oxide mineral insulation. External electrical connection to the emitter is through a leadwire that extends the length of the tube. The leadwire is also insulated along its length using aluminum oxide. A second Inconel leadwire is positioned such that it ends just before contacting the emitter material. This leadwire also extends the length of the sheath in parallel with the emitter leadwire. This leadwire collects a gamma induced current that is nearly identical to the gamma induced current collected by the emitter's leadwire (hence, it serves as a "background" signal lead).

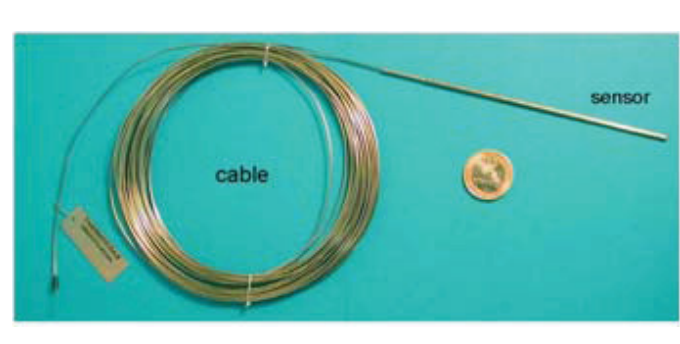

Figure 45. Typical SPND configuration.

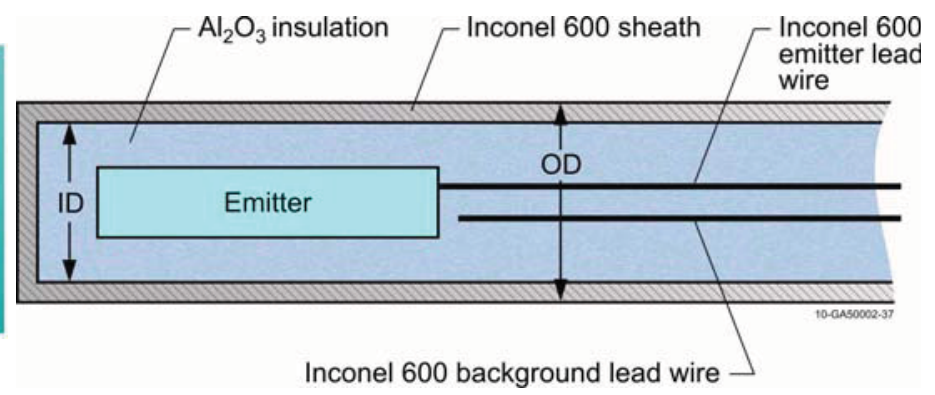

SPNDs are characterized by their response time. Delayed response SPNDs (e.g., vanadium, rhodium, and silver) generate a current of electrons through $(n, \gamma)$ reactions that result in an unstable nucleus. The nucleus subsequently decays by beta emission with a given half life yielding a delayed response for the SPND. In prompt response SPNDs (e.g., platinum, hafnium, gadolinium, and cobalt), currents are generated from gamma interactions producing free electrons through processes, such as internal conversion, Compton scattering, or the photoelectric effect (pair production is a minor contributor). The gammas come from prompt gammas produced by neutron capture in the emitter and from background gammas present in most applications of interest (e.g., nuclear reactor cores). It should be noted that most of the background gamma response of a prompt SPND is due to neutron capture gammas in a reactor core (as opposed to fission product gammas); these capture gammas are also proportional to the neutron flux.

Fission Chambers. For decades, in-pile neutron flux measurements in MTRs were obtained using SPNDs; and signals were generally correlated with post-irradiation analysis of activation foil dosimeters. The development of CEA's sub-miniature fission chambers for in-pile measurements of high thermal neutron fluxes (up to $4 \times 10^{18} \mathrm{n} / \mathrm{m}^{2}$-s) represents a significant improvement. These $1.5 \mathrm{~mm}$ external diameter sensors, containing a U235 fissile deposit, were qualified in the BR2 reactor in the CALLISTO loop between 2001 and 2004, although additional studies are needed to verify sensor robustness. These sensors are now manufactured by the PHOTONIS Company under the name "CFUZ53". 7

In addition, $\mathrm{CEA}$, in collaboration with $\mathrm{SCK} \cdot \mathrm{CEN}$, are developing and qualifying an improved sub-miniature fission chamber system, also known as the Fast Neutron Detection System (FNDS) for measuring fast 
neutron fluxes $(\mathrm{E}>1 \mathrm{MeV}){ }^{7}$ At this time, no other sensor can provide online measurement of fast neutron flux. The FNDS is based on a patented miniature fission chamber with a special fissile deposit sensitive to fast flux with a low thermal contribution, operated in Campbelling mode for a high gamma rejection. Data processing software also calculates online the evolution of the signal given by the system. Major components of the FNDS are shown in Figure 46.
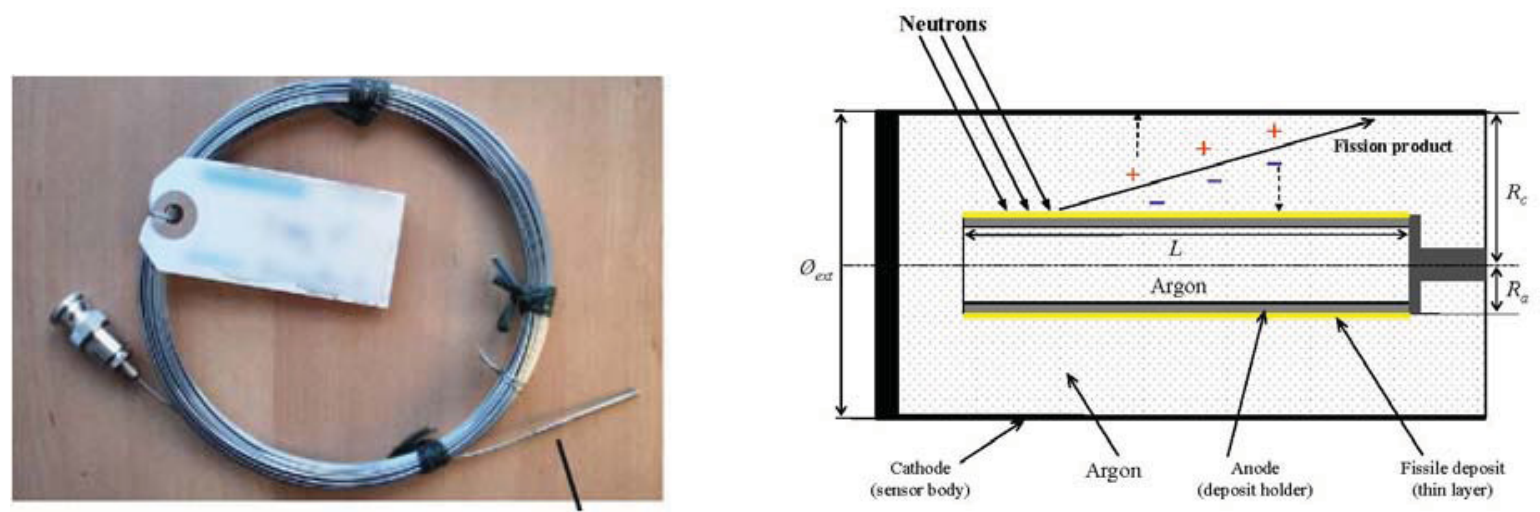

Figure 46. Representative miniature fission chamber configuration.

Selection of the fissile deposit in the fast fission chamber was motivated by two major factors:

- The fission cross-section is much larger for thermal neutrons than for fast neutrons.

- The potential to form other isotopes via radiative capture of (primarily thermal) neutrons. This process, either directly or after some radioactive decays, leads to new isotopes in the deposit that are likely to undergo fission preferentially with thermal neutrons. The sensitivity to thermal neutrons of a chamber based on these isotopes therefore increases gradually. Screen-absorbers, that could prevent thermal neutrons from reaching the chamber, were precluded because of their large size and of the local perturbation induced on the thermal flux.

Analytical simulations of candidate fissile deposits identified ${ }^{242} \mathrm{Pu}$ as the best choice to measure the fast component of a high neutron flux for cases where there is also a significant thermal contribution over the entire reactor cycle. Its sensitivity to fast neutrons is excellent at the beginning of irradiation and slowly decreases with fluence. Prototypes of fission chambers with ${ }^{242} \mathrm{Pu}$ deposit have been manufactured by CEA in Cadarache and tested in 2005 and 2006 in the BR2 reactor by SCK •CEN. As part of an on-going ATR NSUF effort, the use of these fission chambers for ATR applications is being investigated. Initial evaluations began in the ATRC in October 2010.

\subsection{Summary}

As indicated in this section, there is a host of sensors currently available at MTRs that can provide real-time data for key parameters during irradiation. Table 11 summarizes these sensors and the accuracies of sensors deployed or anticipated to soon be deployed in the ATR and HFIR. In general, current MTR instrumentation relies on relatively simple, reliable, compact technologies. Existing efforts are primarily focused on adapting these technologies to the higher temperature/higher flux/higher fluence test conditions requested by ATR and HFIR users. As part of the FCR\&D instrumentation development program, existing instrumentation development efforts will be expanded and include tasks to develop enhanced sensors based on technologies from other industries. Section 5 identifies candidate technologies considered in this FCR\&D instrumentation development effort. 
Table 11. Summary of sensors deployed at or currently investigated for ATR and HFIR.

\begin{tabular}{|c|c|c|c|}
\hline Parameter & Sensor & Operating Conditions & Accuracy \\
\hline \multirow[t]{6}{*}{ Temperature } & Melt Wires & $100-1200{ }^{\circ} \mathrm{C}$ & $\begin{array}{l}\text { 2-3 }{ }^{\circ} \mathrm{C} \text {, but limited by } \\
\text { number of wires with } \\
\text { different melting } \\
\text { points in test. }\end{array}$ \\
\hline & SiC monitors & $100-800^{\circ} \mathrm{C}$ & $2 \%$ \\
\hline & Thermocouples (N, K) & $100-1000^{\circ} \mathrm{C}$ & $2 \%$ \\
\hline & $\begin{array}{l}\text { Thermocouples } \\
\text { (HTIR-TCs) }\end{array}$ & $100-1800{ }^{\circ} \mathrm{C}$ & $2 \%$ \\
\hline & $\begin{array}{c}\text { Thermocouples - Type C, } \\
\text { D, R, and S }\end{array}$ & $100-2000^{\circ} \mathrm{C}$ & $\begin{array}{l}2 \% \text { or higher, } \\
\text { decalibrate due to } \\
\text { transmutation }\end{array}$ \\
\hline & $\begin{array}{c}\text { Ultrasonic } \\
\text { Thermometers }^{\mathrm{a}}\end{array}$ & $1300-3000{ }^{\circ} \mathrm{C}$ & $2 \%$ \\
\hline \multirow[t]{2}{*}{ Thermal Conductivity } & Multiple Thermocouple & $\begin{array}{l}100-3000{ }^{\circ} \mathrm{C} \text {, depending on } \\
\text { thermocouple type }\end{array}$ & $2-8 \%$ \\
\hline & Hot Wire Needle Probe & $\begin{array}{c}100-3000^{\circ} \mathrm{C} \text {, depending on } \\
\text { materials selected }\end{array}$ & $2 \%$ \\
\hline \multirow[t]{2}{*}{ Density / Geometry Changes } & Length - LVDT & up to $500^{\circ} \mathrm{C}^{\mathrm{b}}$ & $1-10 \mu \mathrm{m}$ \\
\hline & $\begin{array}{l}\text { Diameter - Diameter } \\
\text { Gauge }\end{array}$ & up to $500^{\circ} \mathrm{C}^{\mathrm{b}}$ & $1-10 \mu \mathrm{m}$ \\
\hline Crack Initiation /Growth & DCPD Method & $350^{\circ} \mathrm{C} / 2250 \mathrm{psia}$ & $\sim 20 \%$ \\
\hline Young's Modulus & Loaded Creep Specimen & up to $500^{\circ} \mathrm{C}^{\mathrm{b}}$ & $\sim 10 \%$ \\
\hline \multirow[t]{2}{*}{ Fission Gas/Pressure } & Sampling & Numerous isotopes & Unknown \\
\hline & Pressure gauge & $\begin{array}{c}15 \text { bar }(220 \mathrm{psi}) \text { to } 70 \mathrm{bar} \\
(1020 \mathrm{psi})\end{array}$ & $\begin{array}{l} \pm 0.2 \text { bar }(2.9 \mathrm{psi}) \text { to } \\
\pm 0.5 \text { bar }(7.3 \mathrm{psi})\end{array}$ \\
\hline \multirow[t]{3}{*}{ Flux - Thermal } & Flux wires / Foils & Material dependent & $\sim 10 \%$ \\
\hline & SPNDs & Dependent on emitter & $\sim 1-10 \%{ }^{\mathrm{c}}$ \\
\hline & Fission Chambers & Dependent on fissile deposit & $\sim 1-10 \%{ }^{\mathrm{c}}$ \\
\hline \multirow[t]{2}{*}{ Fast } & Flux wires / Foils & Material dependent & $\sim 1-10 \%$ \\
\hline & Fission Chambers & Dependent on fissile deposit & $\sim 1-10 \%$ \\
\hline
\end{tabular}

a. Prior in-pile use typically limited to short duration, fuel damage tests.

b. Some loss of accuracy at $350^{\circ} \mathrm{C}$ due to Curie temperature effects, unless developmental IFE/HRP LVDTs deployed.

c. Accuracy decreases with use. 


\section{CANDIDATE TECHNOLOGIES}

This section identifies other instrumentation techniques, not currently used in-pile, that could improve the fidelity of data obtained during fuel irradiations. Candidate technologies described in this section include those initially identified by INL instrumentation experts and highly ranked input provided by nuclear and non-nuclear instrumentation experts attending a workshop held in November 2010 (see Appendix A). For each candidate technology, experts were requested to provide a representative test capsule design with a description of the sensors that would be installed with their anticipated accuracy for obtaining various material properties of interest. Technology descriptions in this section identify applicable test conditions and benefits of each proposed new technology. Descriptions also identify research and development needed to deploy the new technology.

\subsection{Fiber Optic Sensor Technology}

Optics-based sensing techniques have proven to offer a wide range of capabilities for measuring chemical, physical and mechanical properties of materials through optical spectroscopy and laser ultrasonic methods. Many of these techniques are amenable to fiber optic delivery. Furthermore, fiber optic based sensors capable of measuring strain and environmental conditions, such as temperature and pressure, have become commercially available. The ability to incorporate fiber optic based measurements in ATR and HFIR could allow for in-pile, real-time measurements of radiation effects on materials as well as other environmental parameters.

Fiber optic sensing techniques offer a number of advantages for incorporation into advanced instrumented tests at ATR and HFIR. The optical fibers are small. Common optical fibers measure only $250 \mu \mathrm{m}$ in diameter, allowing as many as 20 fibers to fit into a $1.6 \mathrm{~mm}$ ID tube. Measurement techniques incorporating optical fibers can also be used to measure a variety of material and environmental properties. Optical fibers can also be used in distributed measurement techniques where a property such as temperature or strain can be measured at several locations along a single fiber. Another benefit of fiber optics sensors is that they require no electrical power and don't transmit electrical signals.

\subsubsection{Specific Fiber Sensing Technologies}

A number of fiber optic sensing technologies have been either demonstrated or proposed. Some of these use the optical fiber itself as a sensing element ("intrinsic sensors"), while other methods simply use the fiber to deliver light between the point of interest and the electronics that process the signals ("extrinsic sensors"). Aside from sensing strain, intrinsic fiber sensors are generally used to measure environmental conditions such as pressure or temperature. While these are important parameters, they don't address the measurement of material properties or change of properties due to irradiation. Some relevant fiber sensing techniques are introduced below under the categories of either environmental or material properties sensing.

\subsubsection{Environmental Conditions}

Temperature - Fiber optic sensing of temperature can be performed using a number of techniques. A fiber optic pyrometer is an extrinsic method of temperature measurement in which thermal radiation is collected from the point of interest, transmitted via an optical fiber to an instrument which analyzes the radiation spectrum and thereby determines the temperature. Such devices require a probe at the end of the fiber to maximize thermal radiation collection and would be very sensitive to Radiation Induced Attenuation (RIA) in the transmitting optical fiber. Other fiber optic temperature sensors are either commercially available in some form or have been demonstrated. Extrinsic Fabry-Perot Interferometer (EFPI) sensors determine temperature based on the thermal expansion of a sensing element fused or attached to the end of an optical fiber. Light traveling in the fiber is reflected at both the interface between the fiber and sensing element 
and the back of the sensing element. Using an interferometric technique, the thickness of the sensing element is determined; and the temperature is inferred from the known thermal expansion of the sensing material. Another method of temperature measurement is based on either Optical Frequency Domain Reflectometry (OFDR) or Optical Time Domain Reflectometry (OTDR). These techniques commonly incorporate Fiber Bragg Gratings (FBGs) at various locations along the length of a fiber. Light traveling along the fiber is partially reflected by each FBG. The period of each grating can be determined from the reflected light. Change in the grating period can then be used to determine either temperature or strain. Recently, Luna Innovations has introduced an Optical Backscatter Reflectometer (OBR), a form of OTDR, which relies on the Rayleigh backscatter inherent in the optical fiber itself rather than Fiber Bragg Gratings. With this technique, a backscatter "signature" of the sensing fiber is recorded. Changes to the backscatter signature can then be used to determine the temperature. A unique feature of the OFDR and OTDR techniques is the ability to perform distributed sensing. With distributed sensing, measurement can be made at multiple locations along the length of a single fiber. Distributed sensing would be a strong benefit for in-pile measurements as multiple locations could be monitored from a single penetration.

Pressure - In a technique similar to that described above for single point temperature measurement, an EFPI fiber optic sensor can be used to measure pressure. A small air gap is introduced between the end of an optical fiber and a diaphragm. Deflection of the diaphragm due to external pressure is measured using an interferometric technique, and the pressure is determined from the deflection. Temperature compensation can be incorporated into this technique and accuracies better than $0.5 \%$ of full scale have been demonstrated at pressures up to $500 \mathrm{psig}$ and temperature up to $1000{ }^{\circ} \mathrm{C}$.

\subsubsection{Material Properties}

Strain - Strain in a sample material, when combined with other parameters such as temperature or force, can yield information about the material properties of the sample. Strain in an optical fiber sensor can be sensed in the same manner as previously described for temperature measurement using OTDR or OFDR. Since these techniques directly measure the strain of the fiber, the fiber must be either attached to or incorporated into the sample under investigation. For strain measurements of large structural components, one would not expect the measurement to be significantly affected by the attachment of an optical fiber. However, if the fiber is attached to or incorporated into a small material sample, the effect of incorporating the fiber into the sample must be considered. The method of attachment must also be capable of withstanding irradiation. Measurement of strain via non-contacting methods such as laser ultrasonics is addressed in Section 5.3.

Elastic Constants and Crack Propagation - Measurement of material elastic properties and crack propagation goes to the core of a better understanding of nuclear fuels and materials. Most intrinsic fiber sensors are based on relating fiber strain to the property of interest. As mentioned previously, strain measurements combined with other parameters such as applied force could be used to determine some of these properties. Crack detection is also possible as long as the crack induces a strain in the fiber. However, bonding an optical fiber to a specimen may alter the response of the specimen and the bonding technique would need to survive the radiation conditions in the reactor. Furthermore, detection occurs only along the length of the fiber. Non-contacting methods such as laser ultrasonics and laser based resonant ultrasound spectroscopy are techniques which in principle could yield these desirable measurements. These techniques could possibly be employed using fiber optic delivery and are addressed in Section 5.3.

Thermal Properties - Measurement of thermal properties, such as thermal conductivity, thermal diffusivity, or specific heat, is another area which would be helpful in developing a better understanding of nuclear fuels and materials. Several laser-based techniques for the measurement of thermal diffusivity have been demonstrated. Instruments using laser flash are commercially available and numerous other techniques are 
described in the literature. ${ }^{50}$ Some of these techniques use fiber optic delivery, but incorporation into a configuration compatible with in-pile testing, would require development.

\subsubsection{Limitations and Issues}

A major factor limiting implementation of fiber optic techniques in a nuclear reactor is the deterioration in fiber transmission due to optical damage caused by irradiation. Development of radiation hardened optical fibers has been of much interest to the research community for many years. Short optical wavelengths (from the visible to the UV) appear to be the most dramatically affected by radiation damage. ${ }^{50,51}$ Many optical glass materials darken significantly even under only high flux gamma radiation. ${ }^{1}$ Recent successes in material fabrication have been reported that reduce the radiation sensitivity ${ }^{52,53}$ or that have found wavelength regions around $1 \mu \mathrm{m}$ of reduced sensitivity to damage. ${ }^{54}$

Several optical fibers were recently tested in the CEA OSIRIS nuclear reactor to determine the radiation induced attenuation. ${ }^{55}$ The wavelength region between $800-1200 \mathrm{~nm}$ showed the least radiation induced attenuation. The best fibers showed radiation attenuation of approximately $10 \mathrm{~dB} / \mathrm{m}$ at an accumulated dose of $1.2 \times 10^{21} \mathrm{n}_{\text {th }} / \mathrm{cm}^{2}, 1.3 \times 10^{20} \mathrm{n}_{\text {fast }} / \mathrm{cm}^{2}$, and 16 GGy gamma radiation. Photonic BandGap (PBG) fibers are a relatively new type of optical fiber which guide light on a bandgap principle as opposed to the total internal reflection of standard fibers. Consequently, they can be constructed with a hollow core. A cross sectional view of one such fiber is shown in Figure 47. These fibers showed the least radiation induced attention right up until they ceased to function. The PBG fibers showed less than $4 \mathrm{~dB} / \mathrm{m}$ RIA for a dose of $10 \times 10^{20} \mathrm{n}_{\mathrm{th}} / \mathrm{cm}^{2}$, and $10 \mathrm{GGy}$ gamma radiation prior to failure. Based on published radiation levels in the ATR, one would expect similar attenuation levels after several days in the ATR or HFIR (depending on irradiation location). Luna Innovations has conducted fiber sensor radiation testing in the University of Michigan's Ford nuclear reactor. ${ }^{56}$ In these tests, 14 out of 19 FBG sensors were still delivering readable signals after a dose of $2.0 \times 10^{19} \mathrm{n}_{\text {fast }} / \mathrm{cm}^{2}$ and 0.87 GGy gamma. These fluences correspond roughly to 1 day in a midcore flux trap position in the ATR with the reactor at $110 \mathrm{MW}_{\text {th }}$.

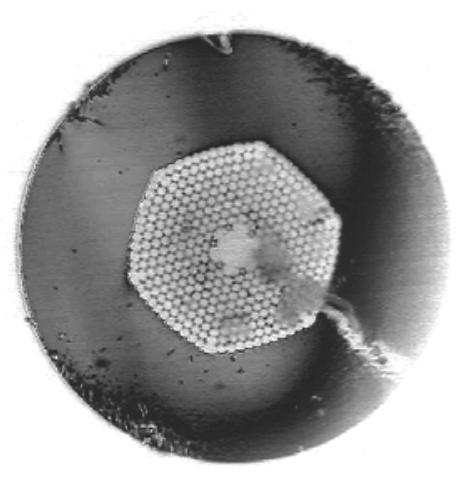

Figure 47. Photonic bandgap fiber.

In addition to the potential for neutron and gamma radiation damage, the optical fibers must also endure the environmental conditions in the reactor. High temperature water has been shown to etch silica. Optical fibers which consist primarily of silica would not last long in the reactor coolant environment if left unprotected. Standard fiber buffer coatings have limited high temperature capabilities or become brittle at higher temperatures which can induce micro-cracks and ultimately, fracture the optical fibers. Metallic coated

1. In a test, optical attenuation increased after extensive gamma radiation at ATR by $50 \% @ 532 \mathrm{~nm}$ and only 1\%@1064 nm in a borosilicate glass provided by S. Raman. 
fibers are available, as are hermetically sealed carbon coated fibers such as those offered by Verrillon. These types of coatings could protect the fibers from the reactor environment, but fiber type and coating would need to be matched to the application for best results. Another option, if compatible with the experiment, would be to seal the fiber inside of a metallic tube so that it is not exposed to high temperature, high pressure water.

\subsubsection{Implementation}

Implementation of optical fibers and optical fiber sensors for in-pile measurements requires special consideration. Optical fibers are fragile and must be handled with care in order to avoid damage or breakage. Outside the reactor, commercially available fiber jacketing should be sufficient to protect the fibers during installation. However the fiber must transition into the reactor to the measurement location. Several methods could be used depending on the measurement requirements. If a distributed sensor, which only measures temperature, is desired, a small diameter steel tube sealed at the bottom could run from the top of the test train down to the measurement location. This tube, if sealed at the top of the test train, could form the pressure boundary such that the optical fiber could be inserted down the tube and even be replaced if necessary. If the optical fiber is required to sense or deliver light to a specific test capsule within the test train, the steel tube could be sealed both at the top of the test train and at the test capsule to provide a conduit for the optical fiber. A fiber optic seal design isolated at the pressure boundary from the high temperature region has been demonstrated ${ }^{57}$ and is shown in Figure 48. This design successfully passed a 3750 psig hydro test for 30 minutes and was later operated at a pressure of $2000 \mathrm{psig}$ and $500^{\circ} \mathrm{F}$ for a period of 10 days without leakage developing at the seal. The standoff tube allowed the seals to be located where the temperature was below the maximum allowable seal temperature.

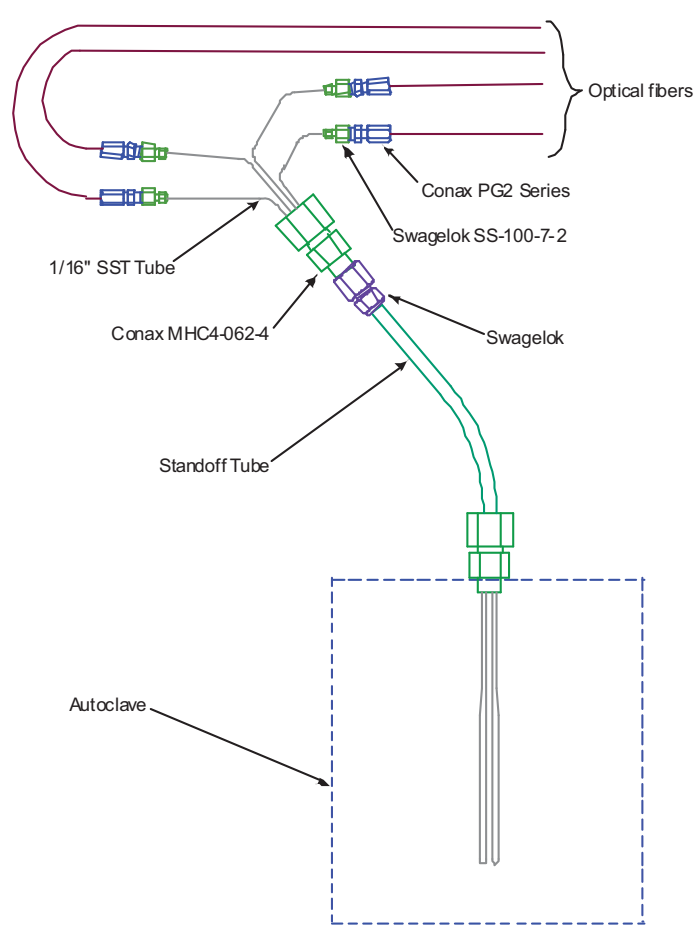

Figure 48. Schematic diagram of the fiber optic pressure boundary seal. 
Fiber optic connections are another consideration, as they can have negative effects on fiber based sensors due to back reflections and loss of light. Connection types and locations would need to be verified prior to integration into the final test assembly design.

\subsubsection{Conclusion}

A number of fiber optic sensing techniques exist for measuring environmental parameters such as temperature and pressure that could be used for in-pile testing. Other optical techniques, which use fiber optic delivery, show promise in measuring meaningful material properties such as crack propagation or elastic constants. The primary limitation to the use of optical fibers for in-reactor measurements is the tendency of optical fibers to darken under irradiation, thereby increasing the attenuation of light propagating in the fiber. Despite this limitation, extrapolating data from radiation testing in other reactors would lead one to believe that optical fibers would last long enough to provide meaningful data for at least the first day and possibly up to several weeks in the radiation levels present in the ATR. In addition, it appears promising that pressure boundary seals for optical fibers exist that would allow optical fiber sensors to be incorporated into ATR instrumented tests.

\subsection{Ultrasonic Transducers}

Ultrasonic techniques for inspection and material characterization using various wave modes have been successfully implemented in a diverse range of forms, and these employ waves with frequencies that can be as low as $\mathrm{kHz}$ or as high as a few GHz. For nondestructive testing/nondestructive evaluation (NDT/ NDE) ultrasonic methods, where frequencies are typically in the 1-10 MHz range, a wide variety of metal engineering products and structures are inspected at the time of manufacture and during in-service. Applications can include pipes, plates, castings, fabrications and the welds which they contain. ${ }^{58}$ In addition, ultrasonic methods are increasingly being deployed to inspect non-metals, such as ceramics and composites, including aero-space and wind turbine applications.

Ultrasonic methods also have a long history of successfully characterizing the properties of solids, liquids and gases. ${ }^{59,60,61}$ They have been demonstrated to accurately measured velocity and determine fundamental physical properties. Data can also, in the case of solids, be used to provide signatures that characterize properties, such as grain size, morphology and damage. An acoustic microscope, operating at frequencies from about $20 \mathrm{MHz}$ to several $\mathrm{GHz}$, has been used to provide images of microstructure at the micron and even sub-micron scale at room and elevated temperatures. Applications of acoustic microscopy have also been successfully performed in post-irradiation examination (PIE). ${ }^{62}$

Ultrasonic methods have been extensively used for process monitoring, measurement, and control. Various research communities have employed ultrasound measurements to investigate complex properties during dynamic processes. Examples include the phenomena encountered in rock physics, where ultrasound up to $1 \mathrm{GHz}$ has been deployed on small samples at both elevated temperature and pressure. ${ }^{63,64,65}$ These methods are also being used to measure properties such as density, viscosity, and particle size in process industries and at the DOE Hanford Waste Treatment Plant (WTP). Such methods are being considered for various applications in fuel reprocessing facilities. ${ }^{66}$

Ultrasonic systems have been tested and deployed for some in-core measurement applications ${ }^{68}$ and for fast reactor under sodium viewing and measurements. ${ }^{69}$ In such measurements, two transduction technologies can potentially be used: one based on the use of piezoelectric materials and the other employing magnetostrictive phenomena. ${ }^{70}$

The basic concept for material inspection and characterization is to use an ultrasonic transducer to generate and detect pulses or waves, which are transmitted into the part being inspected. As the ultrasonic waves travel through the part, features such as dimensions, microstructure and defects, such as porosity or cracks, all affect how the ultrasonic wave propagates. For example, an increase in a length will increase the time it 
takes the ultrasonic wave to travel through the structure or to be reflected back from the surface that has moved. By analyzing the received signal, the microstructure, cracks, dimensional changes, and various other parameters and dynamic processes, including phenomena such as changes in stress or swelling can all be characterized. ${ }^{61,71,70}$ Under suitable conditions, different wave propagation modes have been used to measure a variety of parameters, occasionally even two parameters simultaneously or sequentially. ${ }^{61}$

\subsubsection{Key Benefits}

In-pile ultrasonic measurement technology deployment offers unique measurement opportunities during irradiation testing. Such sensors would allow specimens of interest to be monitored and characterized under prototypical conditions. In addition, such measurements could be performed continuously or at intervals during irradiation, allowing observation of phenomenological changes that occur as a function of time rather than just obtaining data prior to and after irradiation. Continuous in-situ measurement technology could potentially be used to monitor structures or assemblies of interest and lead to the development of in-core structural health monitoring (SHM) technology. Several other industries are already deploying active and passive ultrasonic measurements and monitoring techniques. ${ }^{72,73}$

The dimensions of an ultrasonic transducer that would be deployed in-core in a test assembly will depend on the available space, the specific characteristics of the sample to be characterized, and the wave field that is desired from each transducer (with properties that include the aperture, frequency, and bandwidth, together with the electrical excitation employed). Transducers of potential interest for the proposed applications in-core could be relatively small. Measurement configurations could be pulse-echo (single transducer) or transmission (a pair of transducers). The transducer could also be coupled to a sample in several configurations. A piezoelectric element in a transducer could be air (or gas) backed, giving a high oscillation efficiency or mechanical quality factor, Q, and a narrow bandwidth; or it could employ a backing for damping, to increase bandwidth, to give signals needed to obtain good spatial resolution. ${ }^{70}$ The transducer element can be attached to a buffer rod that is in contact with the sample or applied directly to the sample through a coupling layer or membrane. For example, a $20 \mathrm{MHz}, 3.2 \mathrm{~mm}(\sim 0.125 \mathrm{inch})$ diameter piezoelectric element could be integrated into the $10.0 \mathrm{~mm}(\sim 0.4 \mathrm{inch})$ diameter ends of a typical creep specimen.

Ultrasonic transducers can be utilized in various measurement configurations, depending on the parameters of interest. The microstructure and elastic properties will cause both attenuation and scattering; and the presence of cracks, gaps, and voids will all reflect the sound waves and produce distinct features in the recorded signals. Both material characterization and inspection techniques are already widely used in many industrial applications. ${ }^{61,70,71}$ Ultrasonic temperature measurements have also been demonstrated. ${ }^{61}$ With appropriate temperature compensation, elongation and other dimensional changes, such as swelling, can be measured. Three-dimensional measurements may also be possible with ultrasonic arrays or other techniques. ${ }^{75,76}$

The spatial resolution and accuracy of in-situ ultrasonic measurements is dependent on the design of the transducer, the specimen of interest and the stability of the system under operating conditions (i.e., temperature and radiation). In the rock physics community, measurements have been performed on samples, in the form of cylinders, of the order of 5-10 $\mathrm{mm}$ in diameter and 1-2 cm long, at frequencies as high as 1 GHz. ${ }^{65}$ Measurements at MHz frequencies have also been made on TRISO-fuel compacts in the form of cylinders, of the order of $1 \mathrm{~cm}$ in diameter and a few $\mathrm{cm}$ in length. If discrete reflections are measured, higher ultrasonic frequencies, which have shorter wavelengths, can provide better resolution (perhaps $\sim 1$ micrometer). However, higher ultrasonic frequencies are more susceptible to attenuation mechanisms, such as absorption and scattering, which limit propagation distance.

The optimal design for a particular application will ultimately involve understanding the material under investigation, its microstructure, its attenuation properties, the nature of the degradation or change to be studied, the in-core conditions (flux, temperature, duration and gas/fluid environment), and the desired 
measurement system characteristics; and it will certainly involve engineering trade-offs depending on the desired data, the material properties, and the required accuracy of the measurement.

\subsubsection{Implementation}

These are several possible ultrasonic measurements that could be adapted to fit into assemblies that are deployed for irradiation in the ATR or HFIR. Two possible implementations of ultrasonics that could be deployed in a rod assembly are shown in Figure 49. Figure 50 illustrates a different setup, a stepped transducer configuration, which has been used for fuel plate inspection with a standoff, where a fluid, water or a high pressure gas could be used as the couplant.

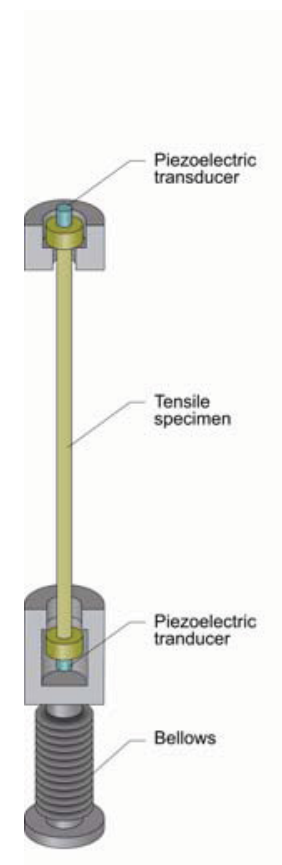

(a)

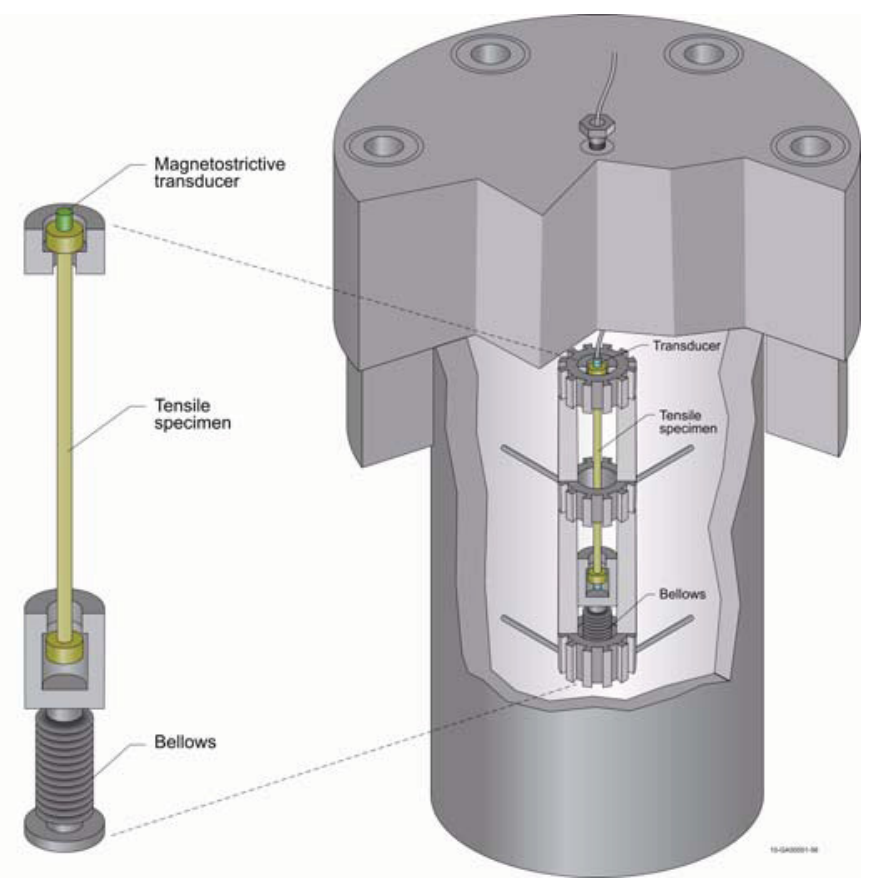

(b)

Figure 49. Ultrasonic in-pile autoclave evaluations using (a) piezoelectric transducers or (b) magnetostrictive transducer on a creep specimen.

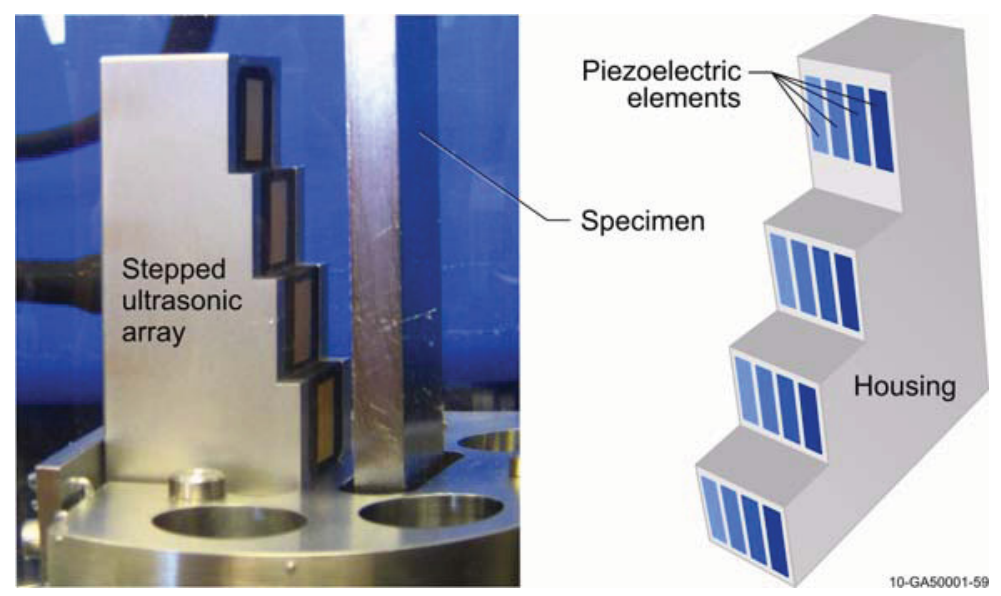

Figure 50. 'Stepped' array for in-situ scanning of fuel plates. 
Concepts where ultrasonic transducers are mounted on the ends of a creep specimen using peizoelectric or magnetostrictive transducer(s) are shown as Figure $49 .{ }^{74}$ In these configurations, the transducer elements are integrated into the creep specimen to form a single unit for in-core testing. As shown in this figure, either through-transmission (two transducer elements as shown in Figure 49a) or pulse-echo (one transducer element as shown in Figure 49b) configurations could be used to measure the length of the creep specimen in-situ by measuring the ultrasonic transit time through the specimen. Likewise, degradation and change in the microstructure can be measured by monitoring signal amplitude and spectral content.

As noted above, a 'stepped' array concept is shown in Figure $50 .{ }^{75,76}$ Such an array of ultrasonic transducer elements could be placed in the reactor coolant pool next to a fuel plate or an aluminum structure. Sound waves reflected from the front surface of the fuel plate could be used to measure bowing of the plate, blister formation, or surface spalling. The sound waves will also penetrate into and interact with features inside the fuel plate. In this setup, cracking, void formation, and swelling are detected with an ultrasonic transducer array by analyzing the recorded signals. ${ }^{75,76}$

In addition to the concepts shown in Figures 49 and 50, there are a range of additional measurements and configurations which could also be deployed, such as those which measure gas concentration/composition in a rod head-space or investigate property changes, due to void swelling in a metal or those in a fuel compact comprised of TRISO fuel particles set in a matrix, as a function of time.

\subsubsection{Current Status}

Ultrasonic measurements in high temperature and high radiation environments began after World War II. The current state of the art has recently been reviewed by Ensminger and Bond. ${ }^{70}$ In assessing the status of this technology applicable to in-core measurements, it is recommended that evaluations be conducted in several areas:

- ultrasonic sensors for detecting temperature, geometry changes, and other phenomena for inert gas testing

- ultrasonic sensors specific to fast reactors (e.g., liquid metal) measurements;

- fundamental ultrasonic piezoelectric transducer studies in high temperature and high radiation environments

The status of each of these areas is described in this section.

\subsubsection{In-Pile Applications}

There are several reports documenting successful in-pile use of ultrasonic thermometers and other types of instrumentation. Arave and Meservey, ${ }^{77}$ Arave, ${ }^{78}$ and Bell et al ${ }^{79}$ describe high-temperature in-pile ultrasonic thermometer applications. Since this time, there have been several studies, ${ }^{80,81,68}$ where acoustic measurements have monitored fission gas release. Most recently, a sensor has been designed for 2011 deployment in the OSIRIS reactor.

Measurements have also been made in the laboratory of materials of potential interest for in-core characterization, including compacts formed from TRISO fuel particles in a carbon matrix which were - about $1 \mathrm{~cm}$ in diameter and $2 \mathrm{~cm}$ long. ${ }^{82}$

\subsubsection{Liquid-Metal Reactor Applications}

Ultrasonic measurements and advanced ultrasonic imaging has also been developed for the hostile environments found in liquid metal cooled fast reactors, where there are intense gamma fields and for sodium 
in hot standby temperatures typically at about $260^{\circ} \mathrm{C}$. The history for fast reactor imaging work has its roots in studies performed at the Hanford Engineering Development Laboratory (HEDL). ${ }^{83}$ In support of fast reactors, a range of ultrasonic tools have been developed and deployed, as reported in Reference 69. Currently, work is on-going in several countries, including the USA, France and Japan. ${ }^{84}$ There is activity to demonstrate transducers for deployment at $450{ }^{\circ} \mathrm{C}$ in a $\mathrm{Pb} / \mathrm{Bi}$ (lead-bismuth) eutectic and deployment in the MYRRHA Reactor in Belgium. ${ }^{85}$ Deployments in high radiation fields have included acoustic emission transducers on the outside of primary pressure vessels and recent in-core instrumentation development, primarily by CEA. ${ }^{68}$

There has been significant experience at high temperatures with piezoelectric transducers that can provide guidance for in-core liquid metal applications. Ultrasonic methods using lithium niobate have been used in non-radiation environments at temperatures up to $1000{ }^{\circ} \mathrm{C}^{86}$ to investigate molten metals and solidification phenomena. ${ }^{70}$ In an effort to overcome challenges associated with providing ultrasonic generation and coupling for extended periods between a transducer and a substrate at high temperatures, researchers have successfully used gold and gold-indium bonding layers at $400{ }^{\circ} \mathrm{C}$ for periods in excess of 400 days. ${ }^{87}$ There are also issues with liquid metals at temperature relating to transducer face material selection to ensure wetting and acoustic coupling in fluids such as liquid sodium. ${ }^{88}$ The ultrasonic characteristics of the three phase material mix in petro-chemical catalysis units have been investigated. Preliminary ultrasonic velocity and attenuation data have been reported ${ }^{89}$ for slurries consisting of water, glass beads, and nitrogen bubbles. This work was then extended to characterization of slurries consisting of molten paraffin wax, glass beads, and nitrogen bubbles at $198^{\circ} \mathrm{C} .{ }^{90,70}$

\subsubsection{Ultrasonic Transducers}

Several studies exist documenting the viability of ultrasonic transducers in intense gamma and neutron fields at elevated temperature. The seminal work is a United Kingdom Atomic Energy Authority (UK AEA) Harwell report by Broomfield. ${ }^{91}$ Various studies have considered both gamma and neutron damage to ceramics. In general, gamma radiation has modest effect on the properties of piezoceramics. ${ }^{92}$ Neutron irradiation does cause significant changes. One series of experiments by Severson ${ }^{93}$ considered the radiation effects on three acoustic emission transducers in neutron flux ranging from $1 \mathrm{x} 10^{11}$ to $2.4 \mathrm{x}$ $10^{12} \mathrm{n} / \mathrm{cm}^{2}$. Changes in both the mean frequencies and response up to $78 \%$ were reported. More recently, Augereau et $\mathrm{al}^{94}$ has reported both gamma and neutron irradiations on piezoceramics, including neutron irradiations with a fluence of $1.6 \times 10^{21} \mathrm{n} / \mathrm{cm}^{2}$ for 150 days. Although some irreversible effects were seen, variations in resonant frequency were less than 1\%. In general, radiation hardened ceramics have lower sensitivity than those conventionally used in NDT.

In recent applications, the majority of in-core and near reactor ultrasonic measurements have used piezoelectric ceramics as the transduction elements. Commercial piezoelectric ultrasonic transducers use a variety of materials, depending on the required operating environment. However, many use lead zirconium titanate (PZT) as the element material. Several studies, as indicated above, have examined the performance of PZT and other lead based piezoelectrics when exposed to radiation and high temperatures. ${ }^{85,92,94-96}$ The lead-based piezoelectric have relatively low Curie temperatures $\left(\sim 320^{\circ} \mathrm{C} / \sim 610^{\circ} \mathrm{F}\right)$. Manufacturers generally recommend a maximum operating temperature of half the Curie temperature $\left(\sim 160{ }^{\circ} \mathrm{C} / \sim 320{ }^{\circ} \mathrm{F}\right) .{ }^{97}$ Therefore, these materials will either not function or function poorly at typical irradiation test temperatures. Furthermore, radiation exposure can significantly decrease the performance of lead-based materials. Baranov et al. ${ }^{96}$ reported an approximately $90 \%$ loss of signal for a fluence of $\sim 1.4 \times 10^{19} \mathrm{n} / \mathrm{cm}^{2}$ thermal neutrons. The various forms of PZT and samples produced using different manufacturing processes do not all exhibit the same response to radiation. Moore et al. ${ }^{98}$ report that thin films (for nonvolatile memory applications) were unchanged due to neutron irradiation to $10^{15} \mathrm{n} / \mathrm{cm}^{2}$. Kazys et al. ${ }^{85}$ suggests that PZTs 
may be operated at higher than half its Curie temperature. However, it is not clear if this would adversely impact the life expectancy of a PZT transducer.

Lithium niobate $\left(\mathrm{LiNbO}_{3}\right)$ is a popular high temperature piezoelectric material that has shown resistance to gamma radiation. ${ }^{85,88,92}$ However, ${ }^{6} \mathrm{Li}$ readily transmutes. A fluence of $2 \times 10^{21} \mathrm{n} / \mathrm{cm}^{2}$ thermal neutrons disintegrates ${ }^{6} \mathrm{LiNbO}_{3}$. The use of ${ }^{7} \mathrm{LiNbO}_{3}$ was reported to have overcome this problem. ${ }^{92}$

Bismuth titanate $\left(\mathrm{Bi}_{4} \mathrm{Ti}_{3} \mathrm{O}_{12}\right)$ is an attractive candidate for use as a piezoelectric material in high temperature environments ${ }^{85,99}$ because its Curie temperature $\left(\sim 650^{\circ} \mathrm{C} / \sim 1200^{\circ} \mathrm{F}\right)$ is approximately twice that of conventional piezoelectrics. Kazys et al. ${ }^{85}$ suggest a maximum operating temperature $\left(\sim 540^{\circ} \mathrm{C} /\right.$ $\sim 1000^{\circ} \mathrm{F}$ ), but this is much greater than half the Curie temperature. However, the bismuth titanate work by Kazys et al. ${ }^{85}$ did not involve neutron irradiation, and no other reference discussing bismuth titinate's response to neutron exposure has been found.

Aluminum nitride (AlN) is also an attractive candidate for use at high temperatures. ${ }^{85,100,101}$ Its piezoelectric properties are derived from its crystal structure, so it maintains its piezoelectric nature up to its melting point. Kazys et al. ${ }^{85}$ recommends a maximum operating temperature of $\left(1100{ }^{\circ} \mathrm{C} / 2000{ }^{\circ} \mathrm{F}\right)$. Their work claims to have exposed AlN to $8.18 \times 10^{18} \mathrm{n} / \mathrm{cm}^{2}$ thermal neutrons without loss of piezoelectric performance. However, this reference only discusses and presents data for gamma radiation doses. It is unclear if the thermal neutron exposure was a separate experiment or occurred simultaneously with the gamma exposure. Even if Kazys et al. were successful at $8.18 \times 10^{18} \mathrm{n} / \mathrm{cm}^{2}$, this is well below typical ATR or HFIR irradiation fluences. Yano et al. ${ }^{102-107}$ tested AlN up to $4.2 \times 10^{18} \mathrm{n} / \mathrm{cm}^{2}(\mathrm{E}>0.1 \mathrm{MeV})$ neutrons, but did not look at piezoelectric properties. Yano et al. noted that there was only a very small change in lattice parameters of the AlN (0.1-0.2\%). Using TEM, Yano et al. also observed dislocation loops (but not voids) formed in the AlN. In addition, Yano el al. commented that the strength of the AlN was reduced. However, it is unclear what impact this would have on piezoelectric transmission.

Several other papers in the literature have examined other characteristics of AlN when exposed to a neutron field. Shikama et al. ${ }^{103}$ investigated the fission gas production of two AlN isotopes. In their paper, they conclude that $\mathrm{Al}_{15} \mathrm{~N}$ may be preferable to $\mathrm{Al}_{14} \mathrm{~N}$ because $\mathrm{Al}_{15} \mathrm{~N}$ is reported to have significantly less helium and hydrogen gas production. More important, Atobe et al. ${ }^{104}$ examined defect formation in AlN. Their data indicate that defects are rapidly annealed. This led them to conclude that the irradiation simultaneously leads to annealing of "induced point defects regardless of the irradiation temperature."

Zinc oxide $(\mathrm{ZnO})$ is another high temperature piezoelectric material of potential interest for in-situ ultrasonic transducers. ZnO's predominate crystal structure (wurtzite) is the same as the crystal structure of AlN. Therefore, $\mathrm{ZnO}$ may be able to maintain piezoelectric behavior up to its melting temperature; and $\mathrm{ZnO}$ 's maximum operating temperature would be similar to that of AlN. However, the transmutation of $\mathrm{Zn}$ must be taken into consideration. ${ }^{64} \mathrm{Zn}$ is known to produce radioactive (primarily gamma) ${ }^{65} \mathrm{Zn}$, which has a long half-life ( $\sim 245$ days). Therefore, a depleted $\mathrm{ZnO}$ would be preferred. It is possible to obtain depleted $\mathrm{ZnO}$. However, it is not clear if depleted $\mathrm{ZnO}$ can be made into a form suitable for use as a transducer. $^{105}$

In addition to the materials for which there are data reporting the effects of radiation, there are also a number of newer materials, many developed to support SONAR or other transduction needs, that have yet to be evaluated in terms of their response to ionizing radiation. 
In Reference 91, Broomfield provides the summary shown in Table 12 of selected properties of several piezoelectric compounds. As shown in this table, this summary includes limiting temperatures and processes for these compounds.

Table 12. Selected properties of selected piezoelectric compounds.

\begin{tabular}{|c|c|c|c|c|}
\hline Compound & $\begin{array}{c}\text { Useful } \\
\text { Temperature } \\
\text { Limit, }{ }^{\circ} \mathrm{C} ; \\
\text { Limiting Process }\end{array}$ & $\begin{array}{l}\text { Electromechanical } \\
\text { Coupling Factor; } k_{t} \\
\text { (thickness direction) }\end{array}$ & $\begin{array}{l}\text { Mechanical } \\
\text { Quality } \\
\text { Factor, } \mathbf{Q}_{\mathbf{m}}^{\mathbf{b}}\end{array}$ & Remarks \\
\hline $\begin{array}{l}\text { Lead Metaniobate } \\
\left.\mathrm{Pb}(\mathrm{Nb})_{3}\right)_{2}\end{array}$ & $<500$; Conduction & 0.6 & $<50$ & $\begin{array}{l}\text { Sintered ceramic. } \\
\mathrm{T}_{\mathrm{c}}=570^{\circ} \mathrm{C}^{\mathrm{C}} . \text { High electrical } \\
\text { conduction above } 250^{\circ} \mathrm{C} .\end{array}$ \\
\hline $\begin{array}{l}\text { Lead Titanate } \\
\mathrm{Pb} \mathrm{Ti} \mathrm{O}_{3}\end{array}$ & $\begin{array}{l}-400 ; \text { Loss of } \\
\text { poling }\end{array}$ & 0.4 & 500 & Sintered ceramic \\
\hline $\begin{array}{l}\text { Quartz } \\
\mathrm{SiO}_{2}\end{array}$ & 300; Twinning & 0.1 & 500 & $\begin{array}{l}\text { Single crystal; } \\
\mathrm{T}_{\mathrm{c}}=560^{\circ} \mathrm{C}\end{array}$ \\
\hline $\begin{array}{l}\text { Tourmaline } \\
\text { Mineral Alumina- } \\
\text { Boro-Silicate Z P late }\end{array}$ & $\begin{array}{l}\text { - 650; Chemical } \\
\text { dissociation }\end{array}$ & 0.07 to 0.11 & 1000 to 5000 & $\begin{array}{l}\text { Natural single crystal. } \\
\text { Synthetic material } \\
\text { unavailable; } \mathrm{T}_{\mathrm{c}}>750{ }^{\circ} \mathrm{C}\end{array}$ \\
\hline $\begin{array}{l}\text { Strontium Niobate } \\
\mathrm{Sr}_{2} \mathrm{Nb}_{2} \mathrm{O}_{7}\end{array}$ & $\begin{array}{l}-330 ; \text { Loss of } \\
\text { poling and hence } \\
\text { piezoelectricity }\end{array}$ & 0.4 & 50 to 300 & $\begin{array}{l}\text { Unilator PC5 Vermitron } \\
\text { PZT 5A; Sintered ceramic; } \\
\text { Ages at } 300^{\circ} \mathrm{C} \text {, but not } \\
\text { seriously } \mathrm{T}_{\mathrm{c}}=365^{\circ} \mathrm{C}\end{array}$ \\
\hline $\begin{array}{l}\text { Lead zirconate } \\
\text { Titanate, } \\
\mathrm{Pb}\left(\mathrm{Zr}_{.05} \mathrm{Ti}_{0.5}\right) \mathrm{O}_{3}\end{array}$ & - 700; Conduction & $\begin{array}{c}0.1 \\
0.4 \\
(0.3 \text { Shear) }\end{array}$ & 1000 to 5000 & Single crystal; $T_{c}=1200^{\circ} \mathrm{C}$ \\
\hline
\end{tabular}

a. $\mathrm{k}_{\mathrm{t}}$ - Electromechanical Coupling Factor is an indicator of the effectiveness with which a piezoelectric material converts electrical energy into mechanical energy, or converts mechanical energy into electrical energy.

b. $\mathrm{Q}_{\mathrm{m}}$-Mechanical Quality Factor is an indicator of the reactance / resistance for series circuit equivalent to the ceramic element.

c. $\mathrm{T}_{\mathrm{c}}$ - crystallization temperature.

Compared to PZT, the temperature and gamma radiation resistant piezoelectric materials $\left(\mathrm{AlN}, \mathrm{Bi}_{4} \mathrm{Ti}_{3} \mathrm{O}_{12}\right)$ are considered 'hard' piezeoelectrics. They have a high mechanical quality factor, low dielectric losses, low permittivity, and a low electromechanical coupling factor. ${ }^{1}$ Table 13 summarizes relevant material properties associated with operational limits for these two materials.

1. A low electromechanical coupling factor indicates that a material is less efficient in converting electrical pulses into ultrasonic waves, but it is more sensitive for detecting ultrasonic waves. 
Table 13. Summary of candidate piezoelectric material properties ${ }^{85}$

\begin{tabular}{|c|c|c|}
\hline Property & Bismuth Titinate $\left(\mathrm{Bi}_{4} \mathrm{Ti}_{3} \mathrm{O}_{12}\right)$ & Aluminum Nitride (AIN) \\
\hline Curie temperature & $650^{\circ} \mathrm{C}$ & $2200^{\circ} \mathrm{C}^{\mathrm{a}}$ \\
\hline Recommended max temperature & $550^{\circ} \mathrm{C}^{\mathrm{b}}$ & $1100^{\circ} \mathrm{C}^{\mathrm{c}}$ \\
\hline Piezoelectic results after gamma exposure & $\begin{array}{l}\text { No change in pulse echo signal } \\
\text { amplitude ( } 22.7 \mathrm{MGy} \text {, room } \\
\text { temperature) }\end{array}$ & $\begin{array}{l}\text { No change in pulse echo signal } \\
\text { amplitude (18.7 MGy, room } \\
\text { temperature) }\end{array}$ \\
\hline $\begin{array}{l}\text { Piezoelectic results after thermal neutron } \\
\text { exposure }\end{array}$ & Unknown & $\begin{array}{l}\text { Claimed } 8.18 \times 10^{18} \mathrm{n} / \mathrm{cm}^{2} \text {, but } \\
\text { paper does not clearly discuss }\end{array}$ \\
\hline $\begin{array}{l}\text { Piezoelectic results after fast neutron } \\
\text { exposure }\end{array}$ & Unknown & Unknown \\
\hline Other & & $\begin{array}{l}\text { Yano }{ }^{102-107} \text { went to } 4.2 \times 10^{18} \mathrm{n} / \\
\mathrm{cm}^{2}(\mathrm{E}>0.1 \mathrm{MeV}) \text { neutrons, but } \\
\text { did not look at piezoelectric } \\
\text { properties }\end{array}$ \\
\hline
\end{tabular}

a. Melting temperature.

b. Based on Reference 85 recommendation.

c. With protection from oxidation.

Customized transducer designs must be developed for any inpile applications. However, several designs have been developed that could form the basis for additional in-pile designs. ${ }^{83,88}$ Transducers have been developed and deployed previously in high pressure gas environments in excess of $1200 \mathrm{psi}^{108}$ With careful design, operation in a 2000 psi environment should be possible.

Magnetostrictive transducers provide an alternate transduction technology. Such transducers were successfully used for ultrasonic fuel temperature measurements. ${ }^{109}$ In addition, these transducers have been used at high temperature to measure ultrasonic velocity in refractory materials ${ }^{110}$ and to determine Young's modulus in $\mathrm{SiC}$ refractory castables at temperatures in excess of $1000{ }^{\circ} \mathrm{C} .{ }^{111}$ In these applications, a magnetostrictive rod undergoes a change in length under an applied magnetic field. A pulsed magnetic field, produced by a coil surrounding the magnetostrictive wire, generates an acoustic pulse which travels down an attached transmission line and sensing element. The coil, in turn, acts as a receiver for pulses reflected at sensor discontinuities. This so-called pulse-echo technique has been used for in-pile fuel temperature measurement as high as $3000{ }^{\circ} \mathrm{C}$. The transducer components, including the coil wire, coil form, and magnetostrictive wire, are readily chosen from materials commonly used in-pile. As shown in Figure 51, Remendur (composition $49 \mathrm{wt} \% \mathrm{Co}, 2 \mathrm{wt} \% \mathrm{~V}, 49 \mathrm{wt} \% \mathrm{Fe}$ ) transducers were installed entirely within fuel rods tested in the Power Burst Facility (PBF). These ultrasonic thermometers operated successfully under irradiation conditions. ${ }^{112,113}$

\subsubsection{Potential In-Core Applications for Ultrasonic Sensors}

As discussed previously, ultrasonics have been extensively used in out-of-pile applications for detecting dimensional changes (both length and diameter), detecting flaws (such as cracks and voids), material characterization, attenuation measurements (velocity - attenuation - modulus, etc), and temperature measurements. Additionally, ultrasonics may be used for a wide range of process, aging, and degradation outof-pile measurements, some of which may be adaptable to in-pile use. ${ }^{61,70}$

Flow measurements have been made by ultrasonic methods for various applications. Measurable flow velocities range from several meters per second in liquids to supersonic levels in wind tunnel tests. Velocity measurements can be made for tube and pipe diameters of less than 1 millimeter and greater than 


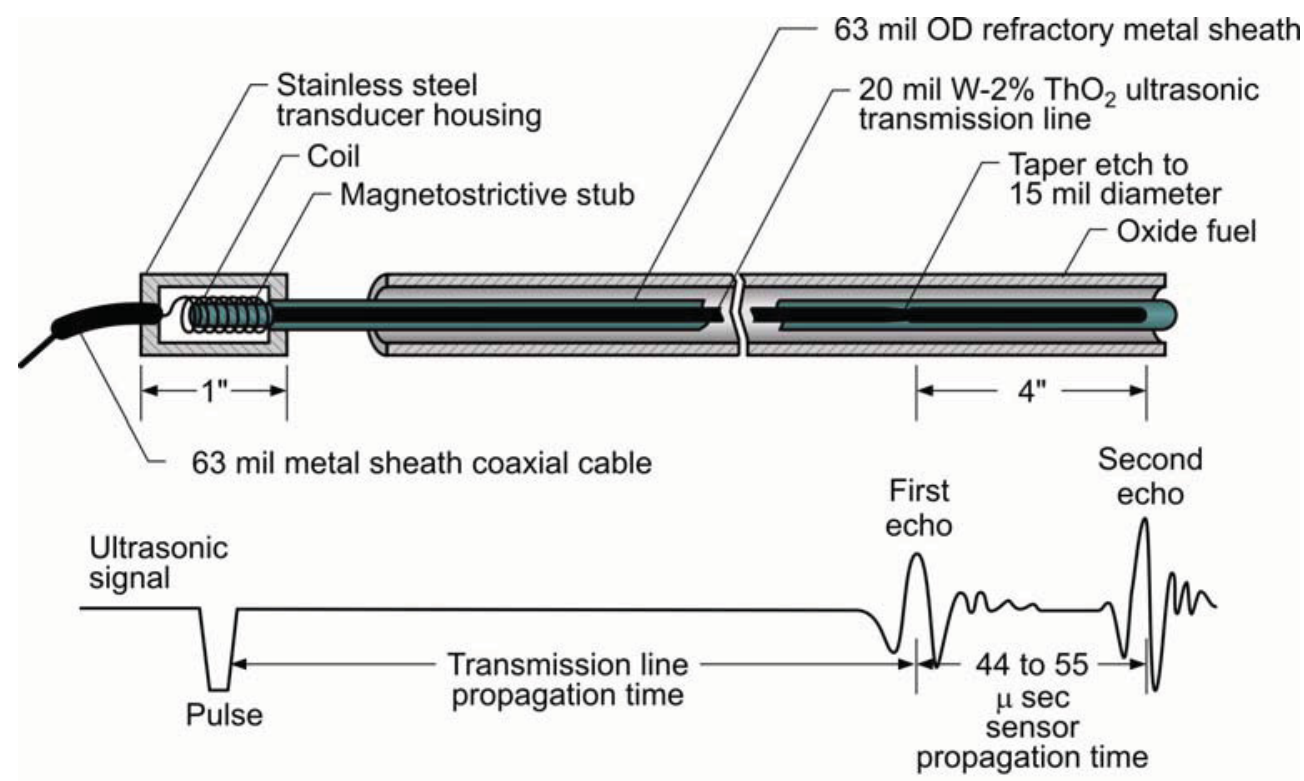

Figure 51. Schematic illustrating PBF UT components and operation.

10 meters, and for open channels (such as a river) more than 1 kilometer wide. Flow measurements can be made at cryogenic temperatures (such as liquid oxygen) and high temperatures $\left(1500^{\circ} \mathrm{C}\right.$ for molten metals). Measurements can be made at near vacuum pressures up to very high pressures (70 MPa for deep sea oil extraction). Measurements may be made actively or passively. Passive flow monitoring consists of "listening" to the fluid flow. Increasing velocity (for a turbulent flow) will result in greater flow noise. Active monitoring may be accomplished in several ways. By measuring the difference in sound velocity of pulses directed upstream and downstream, the flow velocity may be derived. In flows with some particulate impurities, the doppler shift of reflections from those impurities may be used to calculate flow velocity.

Corrosion growth has previously been monitored using ultrasonic means. In prior applications, the acoustic velocity of a thin metallic wire decreased as a corrosion layer developed, with the decrease dependant on the layer thickness. The concept was tested using a zircaloy rod in air at approximately $500{ }^{\circ} \mathrm{C}$. The experimental system worked well until onset of cracking of the corrosion layer. Reported resolution of the test system was approximately $0.05 \mu \mathrm{m} .{ }^{114}$

In a thin rod, Young's modulus is derived using ultrasonics through the following relation: ${ }^{61}$

$E=c^{2} \rho$

where:

$$
\begin{aligned}
& E \quad=\text { Young's modulus } \\
& c \quad=\text { the acoustic velocity of an extensional ultrasonic wave through the sample } \\
& \rho \quad=\text { the bulk density of the sample }
\end{aligned}
$$

Similarly, the shear modulus may be determined by using a torsional wave instead of an extensional wave. Poisson's ratio may be derived from these measurements. Poisson's ratio may also be calculated by using extensional and torsional wave modes simultaneously and measuring the difference in propagation velocities. 
Density measurements in low viscosity (less than 100 centipoise) fluids may be made by immersing a resonator in the fluid. The frequency of resonant vibration is dependent on the fluid density. ${ }^{115}$ Density of higher viscosity fluids may be measured by passing the fluid through a u-tube oscillator, the resonant frequency is again dependent on the fluid density. The density of a non-absorbent solid may be measured using a very thin sheet of the solid material partially immersed in liquid. The amplitude ratio of reflected and transmitted pulse bursts is dependant on the density of the solid. It is unclear if this technique could be expanded to an in-pile method. A substantially simpler method for deriving the density of an isotropic solid is to measure the length change using ultrasonics and assume the change is the same in all directions. If mass is constant, the density change is the inverse of the volume change.

Density, viscosity, and particle size are all measurable using ultrasonic methods. These techniques have been developed for a radiation environment and are being deployed on the DOE Hanford Site, at the Waste Treatment Plant. 66,67

Ultrasonics can be used in the measurement of thermal diffusivity of solids. Techniques for measuring thermal diffusivity typically involve applying heat to a sample and observing the temperature response (the speed of heat flow through the material). Ultrasonics can be used for monitoring the temperature rise of the sample, ${ }^{116}$ as well as for generating the heat. This application of heat to the sample is accomplished by applying focused ultrasound to the surface to be heated. ${ }^{117}$

It is also be possible to measure both gas pressure and composition using ultrasonics. A gas mixture may be driven into resonance using a broadband ultrasonic transducer. The spacing between resonant frequencies of the gas is dependant on the speed of sound in the mixture. The speed of ultrasound through a gas mixture is dependant on the average molecular weight, the average specific heat ratio, and the temperature. Therefore, if temperature is known, the average values for the mixture may be measured; and the composition derived (for known fission gas constituents). For a non-ideal gas, the acoustic velocity is also weakly dependant on pressure. More important, the amplitude of resonant signals in a frequency domain increases with pressure. Therefore, pressure measurements may be made for a calibrated system. ${ }^{118}$

Ultrasonics have been used in studies of grain size in metals, specifically in heat treatment of steels. The metallic grains cause scattering and attenuation of ultrasonic signals. For some (relatively low) ultrasonic frequencies, the absorption and scattering of the signal is strongly dependant on the grain size (attenuation generally increasing with increasing frequency and increasing grain size). ${ }^{119}$ Materials characterization for process control and product conformity, including characterization of microstructure has been reported by Goebbles in Reference 120.

\subsubsection{Technology Readiness and Required R\&D for Deployment}

As noted previously, ultrasonic inspection technology is already widely used in industrial applications for detection of features such as liquid level, cracks, and bonding. ${ }^{61,71}$ This technology has also been extensively used in material characterization. For example, the rock-physics community has made measurements of porosity and fluid properties as a function of temperature, pressure and time. ${ }^{70}$ Although there has been limited deployment of ultrasonic systems for in-pile measurements, acoustic emission transducers are regularly used on the outside of reactor pressure vessels for structural integrity evaluations.

Prior to deploying an ultrasonic technology system to meet FCR\&D program in-pile testing requirements, the main issue to be faced is demonstrating that there are transducer materials which are capable of withstanding the environment within the reactor, more specifically the anticipated temperatures and flux levels in ATR and HFIR fuel irradiations. Ultrasonic thermometers and other measurement systems, previously used for in-pile temperature measurements, provide a starting point for selection of suitable materials.

The open literature on high temperature and radiation resistant piezoelectric materials, particularly when exposed to neutrons is very limited. The limited number of papers report piezoelectric materials exposed to 
neutrons at a flux that is well below typical ATR and HFIR FCR\&D test conditions. In order to deploy in-situ ultrasonic transducer technology, it is necessary to demonstrate the long-term performance and stability of candidate transducer materials under anticipated test conditions. Possible tasks that should be completed to demonstrate the viability of ultrasonics to meet FCR\&D testing requirements include:

- Establish representative fuel compact sample (or samples) sizes and other desired test conditions (e.g., for gas and sodium environments, flux levels, temperature limits, etc.);

- Select an initial target phenomena and measurement configuration for testing (i.e., pulse-echo or transmission);

- Developing a reference case to determine possible accuracy of proposed test configuration to detect desired data in a laboratory environment;

- Develop a customized design that will meet ATR and HFIR geometry constraints;

- Complete laboratory tests to demonstrate possible accuracies and enable the development of an in-core system design. Such tests will enable other elements of the experimental system to be selected and signal processing to be designed. For example, autoclave tests could be conducted on the setup shown in Figure 49 to optimize piezoelectric transducer performance by testing different backing materials and/or joining processes. For fast reactor metal fuels, initial work will leverage on-going work to provide under sodium viewing capabilities, including transducer designs already derived from those developed by HEDL in the 1970's. Laboratory tests should be completed to demonstrate capsule robustness with respect to anticipated test conditions (e.g., with respect to anticipated peak temperature and temperature cycling, sodium [if applicable], PWR pressures [if applicable], etc.);

- In conjunction with the above laboratory evaluations, explore signal processing options to demonstrate sensitivity to phenomena of interest;

- As needed, complete irradiation testing to demonstrate component robustness with respect to gamma irradiation and neutron exposures. The transducer design should be such that parts, in particular the active element, can be interchanged and tests performed on both the candidate element materials (and potentially others, if these can be identified). In this phase, commercial ultrasonic systems would be used for both pulse generation and reception, together with a digital ultrasound system for initial signal processing.

Figure 52 shows conceptual designs for a fuel capsule using ultrasonic techniques to evaluate fuel performance during irradiation. Figure 52(a) includes a piezoelectric transducer; and Figure 52(b) shows a magnetostrictive transducer. Figure 52(b) includes a waveguide, but it could be included in either setup to allow the piezoelectric or magnetostrictive transducer to be remotely located. In this way, the propagation and detection of ultrasonic waves can be studied separately from the survivability of candidate transducer elements. The capsules shown in Figure 52 can be used to measure Young's modulus, grain growth, and density of a single pellet. In Figure 52(b), the magnetostrictive wire and waveguide serve as a pushrod for an LVDT core. For finding Young's modulus, both acoustic velocity and density are required. With this setup, expansion/density would be detected with the LVDT and acoustic velocity with the ultrasonics. If the push rod is sufficiently long (or if the echoes are sufficiently narrow), a notch could be added at the center of the pushrod (so that echoes from the notch and the end of the pushrod could be used for temperature detection (as discussed in Section 4.1). Grain size measurements are based on signal attenuation due to scattering and absorption of ultrasonic energy. Using two transducers might yield a stronger signal and better resolution for grain size detection. 


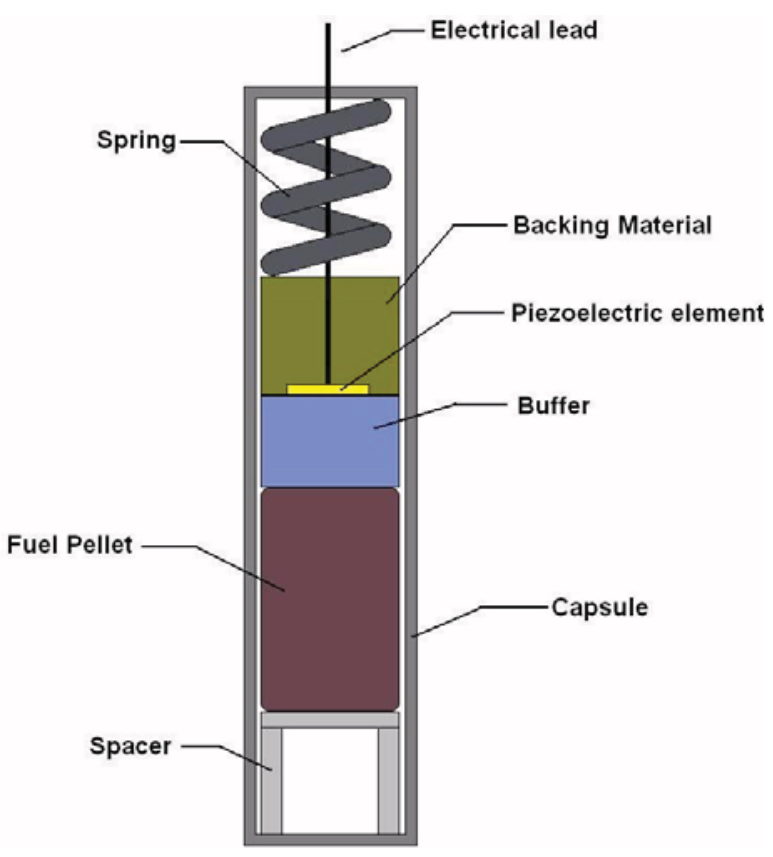

(a)

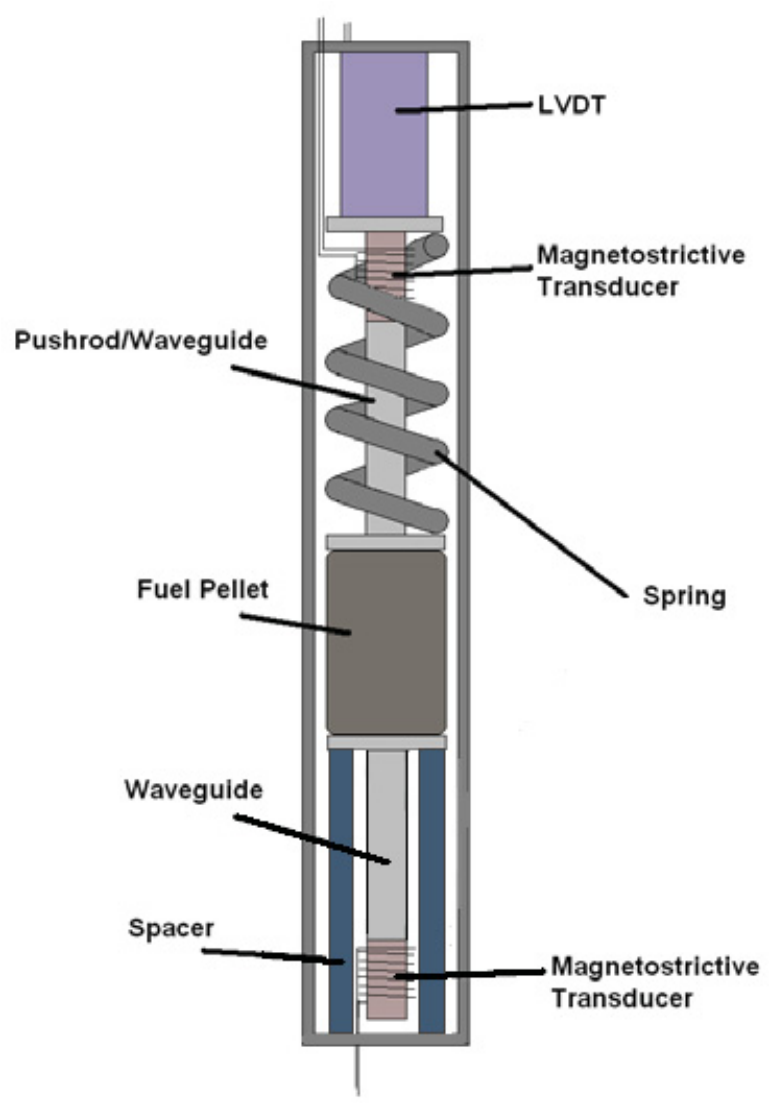

(b)

Figure 52. Potential capsule configuration for in-situ studies of ultrasonic transducer materials and wave propagation.

Once suitable transducers and a capsule design have been demonstrated in the laboratory and their advantages for different test materials and conditions determined, additional R\&D will be required to verify that the proposed setup is optimized for in-pile applications.

\subsection{Laser Ultrasound}

In nuclear reactors, mechanical properties are affected by the interaction of radiation defects with existing defect structures such as dislocations and grain boundaries. These interacting microstructural features involve a perturbation of the perfect lattice (i.e., strain fields). Viewed in this context, high frequency strain waves (i.e., ultrasound) provide a natural choice to study microstructure mediated mechanical properties. Ultrasonic techniques have long been used to assess changes in the microstructure of industrial materials. For example, techniques have been developed to monitor acoustic attenuation due to thermal aging embrittlement, ${ }^{121}$ residual stress, ${ }^{122}$ and changes in texture. ${ }^{123,124}$

Laser acoustics, with optical acoustic generation and detection, has unique advantages over piezoelectric or magnetostrictive methods-it is noncontacting, requires no couplant or invasive sample preparation, and has the demonstrated capability to probe microstructure on a submicron scale. As a consequence, laser techniques are highly reproducible, enabling sophisticated, microstructurally informed data analysis. Generation of ultrasound is accomplished by irradiating a specimen with a short pulse ( $\sim 10 \mathrm{~ns})$ from a high intensity laser. A portion of the incident energy is absorbed giving rise to a rapid and localized thermal expansion of the sample (thermoelastic generation is dominant in metals), which in turn generates ultra- 
sonic waves that propagate through the material. The manner by which ultrasonic waves are generated and propagate depend on the mechanical properties of the material (e.g. texture evolution, void formation, dislocation density, dislocation length, dislocation pinning strength.). Complementing ultrasonic generation, laser-based interferometry can be used to detect ultrasonic waves after propagating through a sample (bulk waves) or along the surface of a sample (surface acoustic waves). Since light is being used for generation and detection of the ultrasonic wave, the specimen being examined is not mechanically coupled to the transducer. As a result, laser ultrasound can be carried out remotely, an especially attractive characteristic for in situ measurements in severe environments. The contactless nature also facilitates attenuation measurements since acoustic coupling to the environment can be limited.

In this section, a brief review of two laser-based ultrasonic techniques is given. Potential applications to in-reactor measurement of microstructure evolution are briefly discussed. At the conclusion of this section, a conceptual sketch for implementation of laser ultrasound in a test capsule at ATR or HFIR is presented.

\subsubsection{Laser-based Resonant Ultrasound Spectroscopy}

Resonant ultrasound spectroscopy ${ }^{125-127}$ involves the study of the mechanical resonances of solids. The resonant response of a particular object depends on the shape, elastic constants, density, and ultrasonic dissipation. Of these properties, the elastic constants and ultrasonic dissipation have been shown to be strongly influenced by microstructure evolution under irradiation.

The low frequency version of this approach was used extensively from the 1950s through the early 1970s to investigate the interaction of radiation defects with dislocations. One of the first examples involves the work of Thompson and Holmes. ${ }^{128}$ They measured the low frequency mechanical resonances of high purity copper samples exposed to neutron irradiation. Their results showed a marked increase in Young's modulus and a decrease in attenuation with increasing radiation dose. Using the oscillating dislocation line model of Granato and Lucke ${ }^{129}$ to interpret their results, it was shown that the change in modulus and attenuation could be related to dislocation pinning by radiation defects. Many other studies have investigated dislocation pinning by radiation defects using temperature as a parameter. ${ }^{129-134}$ While these studies have confirmed the basic elements of the explanation presented by Thompson and Holmes, wide disagreement between experimental results has precluded specification of the type and size of the defect responsible for pinning. This limitation is most likely due to uncertainty in the initial defect structure. The most detailed studies of that era used electrical resistivity to measure vacancy defects and etch pits to measure dislocation concentration.

Advancements made in laser ultrasonics, electron microscopy and model development make a compelling case to advance understanding of ultrasonic interaction with radiation-damaged microstructure. As an example of the potential of laser ultrasound to probe material microstructure, consider recent work at INL involving laser-based resonant ultrasound spectroscopy (LRUS). ${ }^{135,136}$ This new approach, involves exciting a broad spectrum of resonant modes with a pulsed laser and measuring the ring down with a separate laser interferometer. In the current example, LRUS was used to examine in situ changes in microstructure as a rolled copper sample was annealed at high temperature to produce a recrystallization microstructure, (see top of Figure 53). The experimentally determined position of a single ultrasonic resonant peak as a function of increasing temperature is shown in the bottom of Figure 53.

The predicted mode frequencies before and after annealing (also shown in the lower panel of Figure 53) were obtained using the polycrystalline averaged elastic stiffness tensor ${ }^{136,137}$ derived from the EBD data. This close comparison between predicted and measured mode location illustrates that the large shift in frequency is almost entirely driven by a change in texture. Viewed in this manner, LRUS is capable of providing real-time, spatially averaged EBD data which can be used as a direct validation metric for mesoscale models of grain boundary migration and grain growth. 


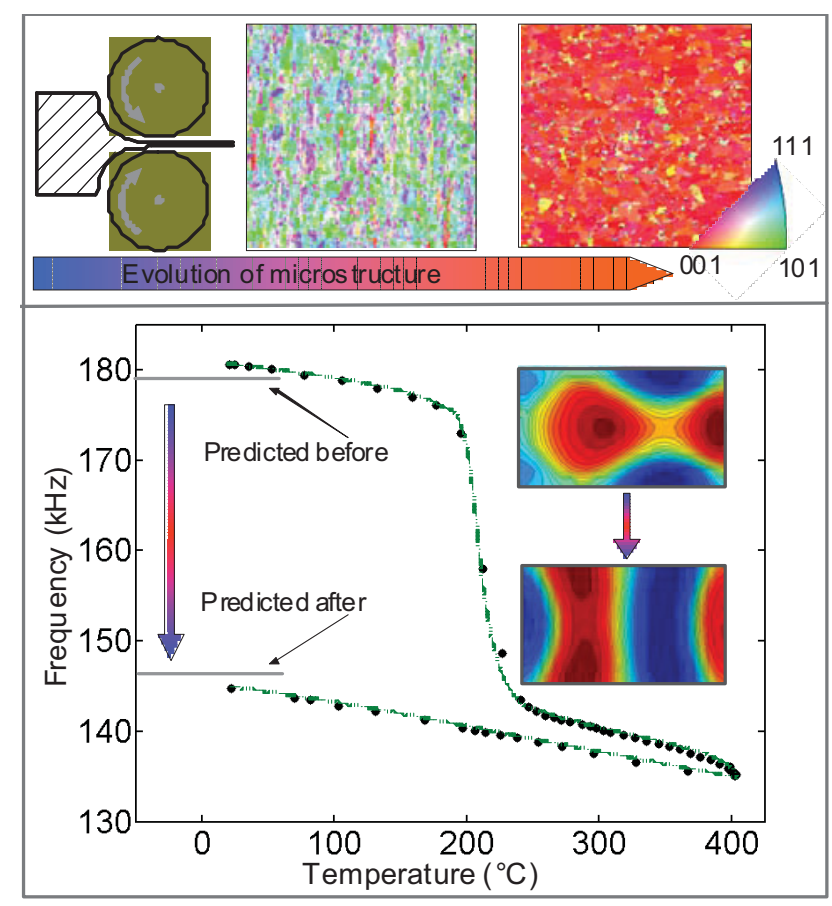

Figure 53. Resonant ultrasound used to provide in situ validation metric. The upper figure shows an electron backscatter diffraction (EBD) rendering of rolled and re-crystallized microstructures. The lower figure shows the change in the location of a single resonant peak during annealing. The data can be used to map the evolution of the elastic stiffness tensor during high temperature processing.

\subsubsection{Imaging Surface Acoustic Waves}

Enhanced resolution and positioning accuracy offered by laser ultrasound in combination with new microstructure imaging technologies have enabled researchers to capture the interaction of ultrasonic waves with isolated microstructural features. This approach is a departure from typical ultrasonic approaches that provide a response averaged over many microstructural features. Imaging ultrasonic interaction with isolated features has enabled, for the first time, a microstructurally informed first principle analysis of experimental data. A striking example of this new direction in ultrasonic imaging is provide by the recent work of Chilo and Zolotoyabko involving the stroboscopic x-ray imaging of surface acoustic wave interaction with a single dislocation in a piezoelectric ceramic. A detailed analysis permitted determination of the local velocities of vibrating dislocations and their viscosity coefficients.

As another insightful example, consider a recent investigation performed at INL involving the imaging of GHz surface acoustic wave propagation across an isolated grain boundary (see Figure 54a). ${ }^{138}$ Surface acoustic pulses were thermoelastically excited in a high purity $(5 \mathrm{~N})$ polycrystalline copper sample. The generation laser pulse was focused at grazing incidence through a microscope objective, and out-of-plane surface motion was recorded with a normal incidence probe beam interferometer. Full animation (not shown here) was obtained by taking images at increasing pump/probe delay times. The location of the grain boundaries along the bounding surface was revealed by lightly etching the sample in a dilute mixture of hydrochloric and hydrofluoric acid. The concentric wavefronts, shown in Figure 54a, arise because of the $76 \mathrm{MHz}$ periodic excitation of the laser source, and the convoluted shape of the wavefront is due to elastic anisotropy. The orientation of the crystallites in the region of interest was obtained using electron backscatter microscopy. A small dashed square superposed on the electron backscatter image (see Figure 54b) corresponds to the data presented in Figure 54a. Due to the large misorientation between the two 
crystallites, the ultrasonic wavefronts exhibit considerable refraction (see dashed box in Figure 54a). However, there is very little reflected ultrasonic energy, which through detailed modeling gives a strong indication of the subsurface grain boundary orientation. It is important to note that it is practical to use surface waves since ultrasonic measurement can be combined with electron backscatter micrographs to identify and isolate specific grain boundaries.

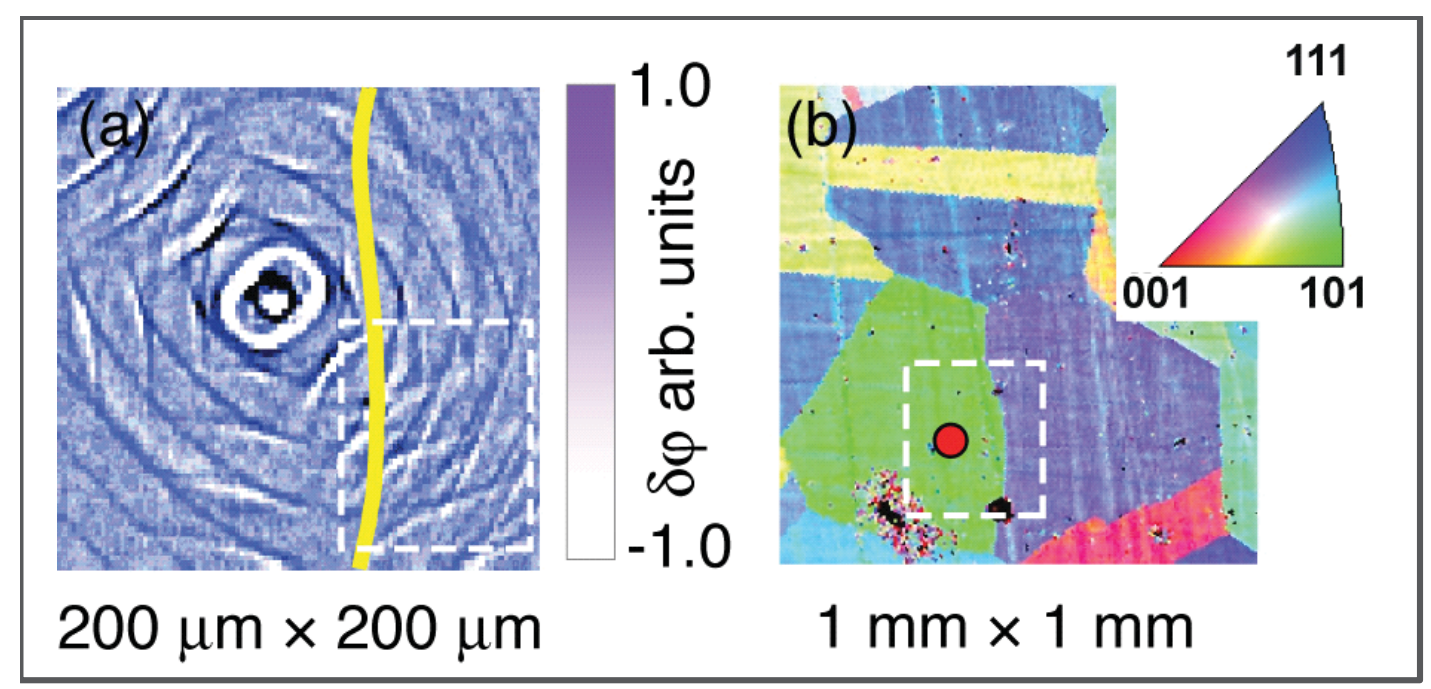

Figure 54. Results from investigation of surface acoustic wave propagation across an isolated grain boundary: (a) Image of surface acoustic phonon pulses propagating across a grain boundary (yellow line) in $\mathrm{Cu}$; (b) Electron backscatter micrograph of region of interest.

\subsubsection{Implementation of In-reactor Monitoring}

In this section, two potential in-pile investigations of microstructure evolution using laser ultrasound are discussed. As an illustrative example, consider monitoring the redistribution of porosity in sintered ceramics immediately after fuel insertion. This type of evolving microstructure will lead to porosity driven variations in the acoustic velocity, which can be monitored in-reactor using laser ultrasonics.

- Porosity redistribution will lead to porosity driven variations in acoustic velocity which will in turn lead to a change in the resonant spectrum of the sample. Microstructurally informed continuum models can be used to define the radial distribution of porosity. The appeal of this approach is that it can serve as a direct validation metric for mesoscopic models of pore transport.

- Megahertz and gigahertz frequency surface acoustic waves can be used to image changes in microstructure mediated mechanical properties. While similar to the LRUS, this approach gives a more direct measure of spatial variations in microstructure. Again, porosity redistribution in a sintered ceramic fuel provides an elucidatory example. In this case, spatial variation in acoustic velocity can be directly related to variations in porosity distribution.

\subsubsection{Implementation into an Irradiation Test Capsule}

Key to the successful implementation of in-pile laser ultrasonics is the design of a test capsule that enables effective optical delivery to and from the sample for both ultrasonic generation and detection. Furthermore, the sample must be contained in an inert gas environment to avoid complications associated with liquid immersion and surface corrosion. Optical fibers are a natural choice for delivering light from the lasers located in the gallery to the test train to be inserted into the reactor. The optical fibers themselves could be 
routed through small diameter stainless steel tubing to both protect the fiber and allow a pressure boundary seal to be created using Conax type fittings. Fiber optic connections could be used in the fittings.

The functional requirements of the test capsule would be to accept the optical fibers, hold and position the sample in a manner which would allow for measurements to be made, provide for light delivery to the sample, collection of the detection laser light, seal against the high pressure coolant, and provide cooling of the sample if necessary.

Conceptually, the test capsule would consist of a small sealed container. The optical fibers enclosed in metal tubing would enter at one end where the metal tubing would be sealed to the container wall. The sample would be held in an inert gas environment in a manner that would not impede optical access and minimize mechanical constraint. There are several options for delivering the light to the sample. The best method would depend on factors such as detection methodology and expected test duration. Radiation induced darkening of optical fibers and optics would limit the time these components could remain in the reactor core. As discussed in Section 5.1, optical fiber darkening is not expected to preclude measurements for at least the first 24 hours of irradiation. A simplified test capsule layout is shown in Figure 55.

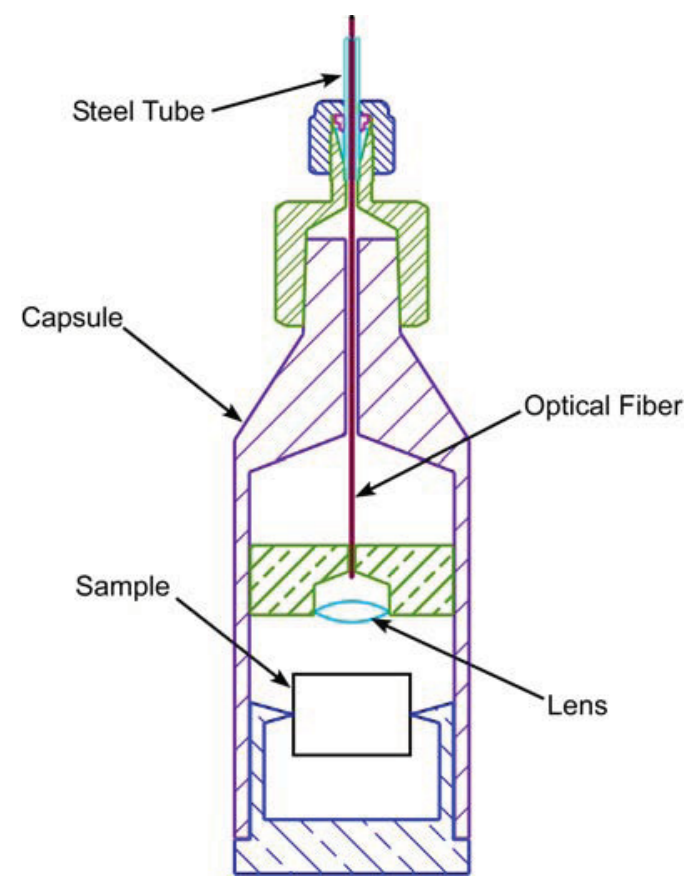

Figure 55. Conceptual sketch for implementing laser ultrasound in an irradiation test capsule.

For extended duration tests, it may be possible to develop a much longer test capsule that would effectively keep the fiber and optics out of the high radiation area associated with the reactor core. In this case the test capsule would be located near the top of the core with the bottom of the capsule in the core area and the top of the capsule above the core. A longer focal distance lens located near the top of the capsule would focus the light from the optical fiber onto the sample located near the bottom of the capsule. The focal length would be sufficient to keep the fiber and optical components in the region above the core where radiation levels are lower and would extend the measurement duration

\subsubsection{Current Status and Required R\&D for Deployment}

To summarize the current status of this technology, the following points should be noted:

- Measurements are routinely made on metallic samples in a laboratory setting; 
- Technique is used to determine microstructure mediated elastic properties.

Some of the engineering research and development issues required for deployment are:

- Verify measurements can be made in a configuration compatible with an ATR or HFIR test train;

- Control of light: spot size, location control, collection;

- Capsule assembly;

- Darkening of fiber;

- Issues associated with capsule seals and fiber optic connectors.

Some of the metrology research and development issues required for deployment are:

- Spectral reflections off polished surfaces are required;

- Efficient ultrasound generation requires strong optical absorption;

- Data Analysis - verify desired properties can be determined from data;

- Determine what test makes the most sense: 1-time domain laser ultrasonics or 2-frequency domain resonant ultrasound.

\subsection{Borescope}

A borescope is an optical device which allows visual inspection of areas that are inaccessible by other means. Both rigid and flexible designs are available, differing in the method of transmitting the image. Rigid types typically transmit the image from one end to the other through a series of lenses and optics. A schematic of a rigid borescope is shown in Figure 56.

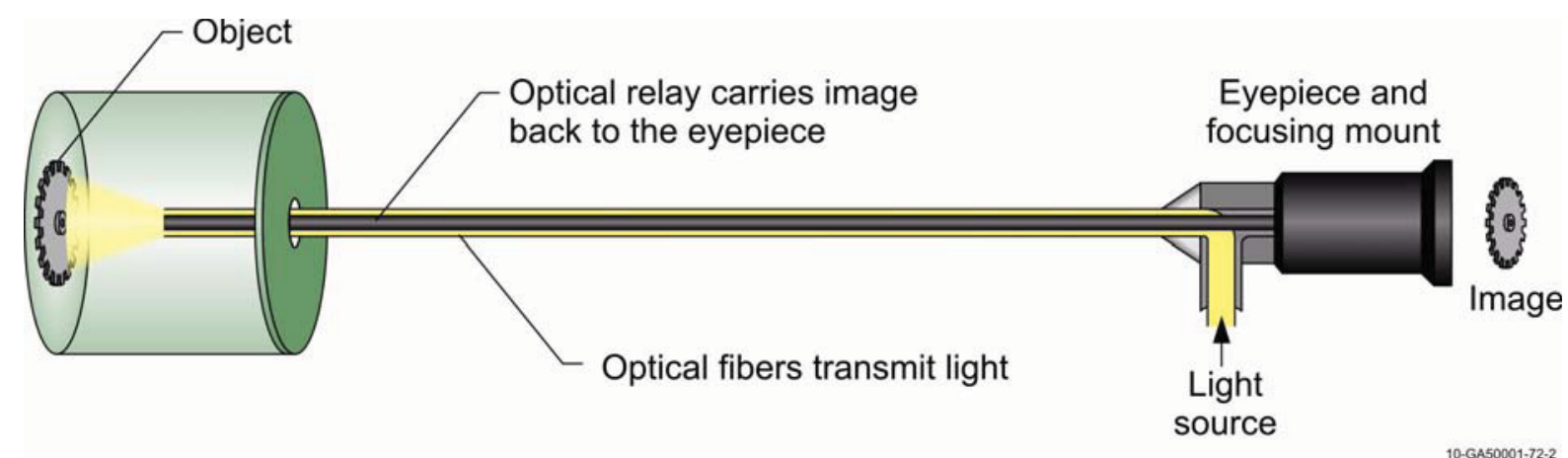

Figure 56. Typical borescope components.

Flexible borescopes can be bent and routed around obstacles and do not required a direct line of sight. They incorporate a coherent bundle of optical fibers that transmit an image from one end of the fiber bundle to the other. Each fiber represents one pixel in the image. Commercially available flexible borescopes may incorporate up to 30,000 individual optical fibers. ${ }^{139}$ Typical fiber bundles range in diameter from 0.35 to $8 \mathrm{~mm}$ and are available in lengths up to $15 \mathrm{~m}$ (50 feet) for non-articulating types. ${ }^{140}$

In a typical borescope design, some of the outer fibers are used to transmit light to the inspection area. A lens collects the light reflected from the object of interest and creates an image on the object end of the fiber bundle. The image is then transmitted pixel by pixel to the opposite end of the fiber bundle where 
additional optics present the image on the end of the fiber bundle to the viewer or onto a charge-coupled device (CCD) camera for viewing on a monitor or computer screen.

The attraction of applying borescope technology to in-pile measurements is the ability to generate a real time visual image of a process or material change as it occurs in the reactor. The borescope would provide a "window" through which one could perform a real time visual inspection. With sufficient resolution and magnification, one could possibly see the development of cracks, porosity or other visually discernible processes.

\subsubsection{Limitations}

Although borescopes are a proven commercially available instrument, there remain a number of limitations and issues to be considered for in-pile use. While a simple borescope could provide a qualitative image of a particular process, it may be difficult to quantify the observed phenomena from the image. For example, it may be possible to visualize the development of a small crack on the surface of the sample, but determining the length of the crack would have to be estimated from the known field of view or other features of known size. Resolution is another issue that would need to be addressed. Rigid borescopes offer better resolution, but may be difficult to implement because they require direct line of sight. In a flexible borescope, the image is transmitted pixel by pixel, so the resolution is limited by the ratio of the pixel size to the image size; and the image size is related to the desired field of view. Thus, there is a trade-off between the discernible feature size and the field of view. Commercial borescope specifications list flaw detection sizes of $0.001 "(25 \mu \mathrm{m})$ for rigid types and $0.005 "(127 \mu \mathrm{m})$ for flexible types. Figure 57 shows an image from a fiberscope where individual pixels can be discerned with close inspection.

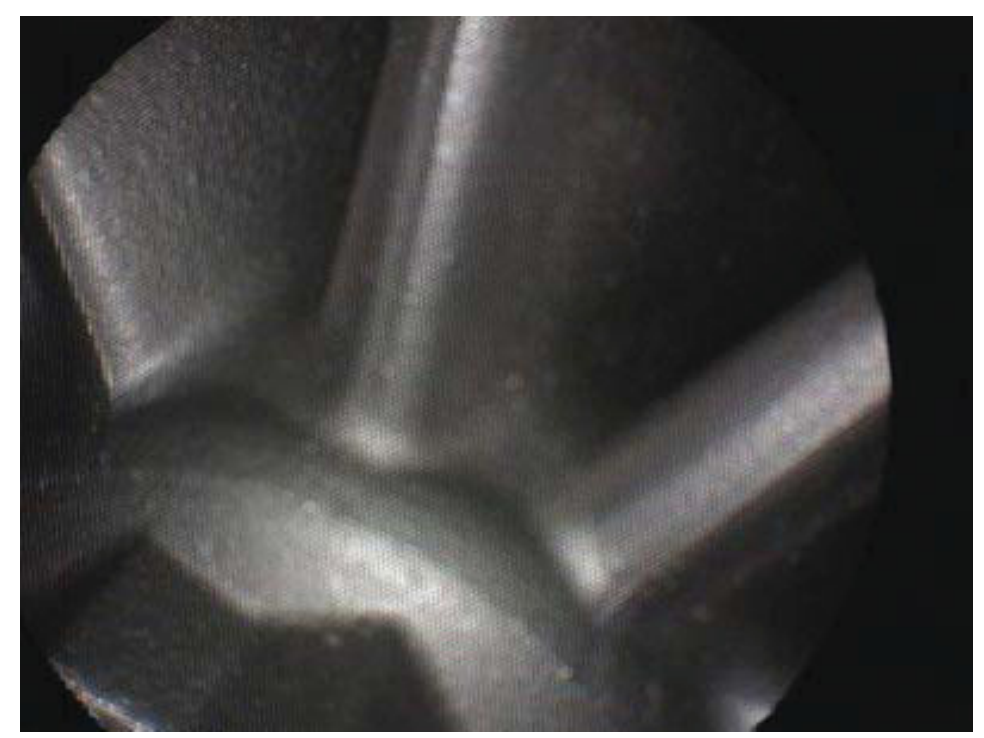

Figure 57. Fiberscope image illustrating individual pixel limitation on resolution. ${ }^{141}$

The optical fibers making up the fiber bundle would also suffer from the same darkening issues as regular optical fibers as would the imaging lens. Existing borescope fiber bundles are not optimized for radiation resistance or the wavelength of light for optimal fiber longevity in high radiation areas. Custom fibers would likely be required. Another issue is the desired sample environment. An inert gas environment would be optimum for light transmission from the object to the fiber bundle, but it may be possible to use a borescope in some cases where the sample is submerged in a coolant. Note that the optical properties of the coolant would effect the ability to capture light from the sample, and contaminants in the coolant could 
also coat the optics limiting light transmission. Hence, this system would not be viable in a sodium-filled capsule.

\subsubsection{Implementation}

Implementation of borescope technology for in-pile measurements would require customizing the basic concept used in commercially available systems. As mentioned previously, a custom fiber bundle would likely be required; and the resistance to radiation darkening of the both the fiber bundle and lens would need to be determined. The fiber bundle would also need to be protected from the environmental conditions inside the reactor. A metal tube or sleeve could be used for this purpose. Depending on the sample environment, the object imaging lens could serve as the cap to seal the protective tube or if an inert environment test capsule is used, the lens could be positioned inside the capsule which would be sealed directly to the metal tube. The metal tube would also serve as the pressure boundary. At the anterior end, the image on the end of the fiber bundle would be focused onto a micro CCD camera. The image from the CCD camera could be viewed and recorded in real time. Standard electrical connectors for power and signal to the camera could be used to allow for installation and removal of the test train. Several borescope vendors offer services for customizing a design for a particular application, so it may be possible to collaborate with a vendor who has expertise in the area in order to speed the development time. A concept for a test capsule incorporating a borescope is shown in Figure 58.

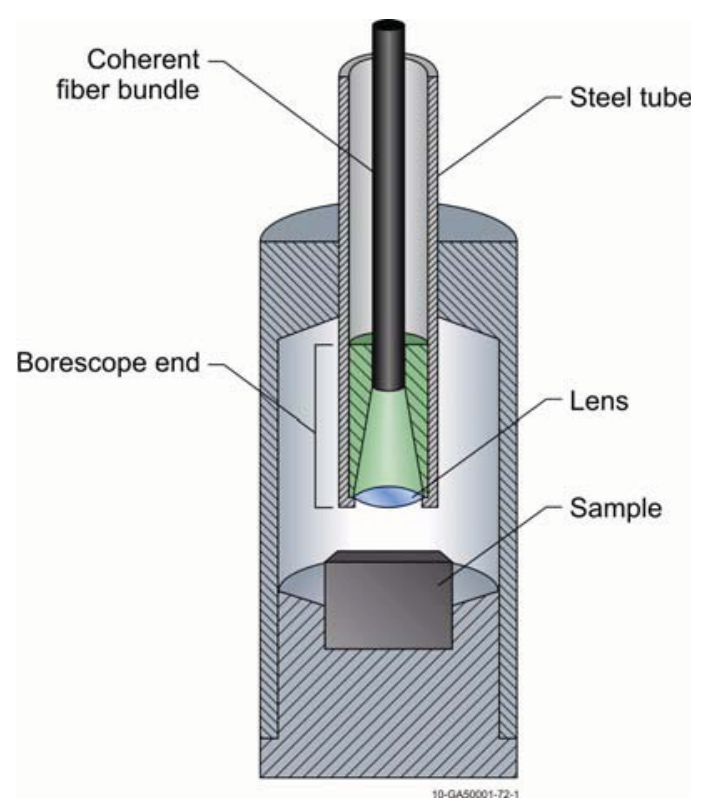

Figure 58. Concept for implementing a borescope into an irradiation test capsule.

\subsubsection{Summary}

Borescope technology could be used to provide a qualitative visual image of a process or change occurring to a sample inside of a reactor. Borescope technology is quite mature, and there are a number of vendors offering borescopes for commercial and industrial applications. The in-pile environment and constraints would require customizing or redesigning commercially available borescopes. The radiation resistance of the coherent optical fiber bundle and imaging lens needs to be determined to have a realistic idea of the longevity of the device inside the reactor. The trade off between resolution and the field of view would also need to be determined and optimized for a particular sample of interest. 


\subsection{Laser-induced Breakdown Spectroscopy}

Laser-induced breakdown spectroscopy (LIBS) is a technique within the class of atomic emission spectroscopy that uses an energetic laser pulse as the excitation source. ${ }^{141-147}$ The laser is focused to obtain an intensity which atomizes and excites the samples surface layer and will form a plasma. In theory, LIBS can evaluate any matter regardless of its material state, solid, liquid or gas. Since all atoms emit light of characteristic wavelength when excited to plasma generating temperatures, LIBS can identify all elements. The element detection range of LIBS is limited by the practical design of the measurement system that includes the laser power, sensitivity of the detector and wavelength range of the spectrograph. The actual detection limits are a function of: a) the plasma temperature; b) the light collection aperture; and c) the intensity in the line of the selected transition. LIBS is a form of optical emission spectrometry and is nearly identical to arc/spark emission spectroscopy.

LIBS can be used to determine the elemental composition of materials. The accuracy of LIBS measurements is normally better than $10 \%$ and the precision is normally better than $5 \%$. Detection limits of 1 to 30 ppm by mass are typical. By taking data at multiple locations, concentration gradients can be determined. Specific to nuclear applications, LIBS can also be used to determine the oxygen to mixed oxide fuel ratio. The LIBS analytical technique is cost effective as well as relatively easy to use and implement. By using a double-clad fiber and a specially designed focusing lens, a single fiber can be used to deliver the excitation light and collect the characteristic atomic emission lines. This enables a compact sensor head that ensures the collection optics are always in alignment with the light generating plasma.

LIBS functions by focusing a laser onto the surface of the specimen as shown in Figure 59. The laser ablates a thin layer of material, on the order of nanograms to picograms. This ablated material creates the plasma plume that has temperatures in excess of $100,000 \mathrm{~K}$. On average, when local thermodynamic equilibrium is reached, plasma temperatures range from 5,000-20,000 K; and this is the usual regime to collect data.

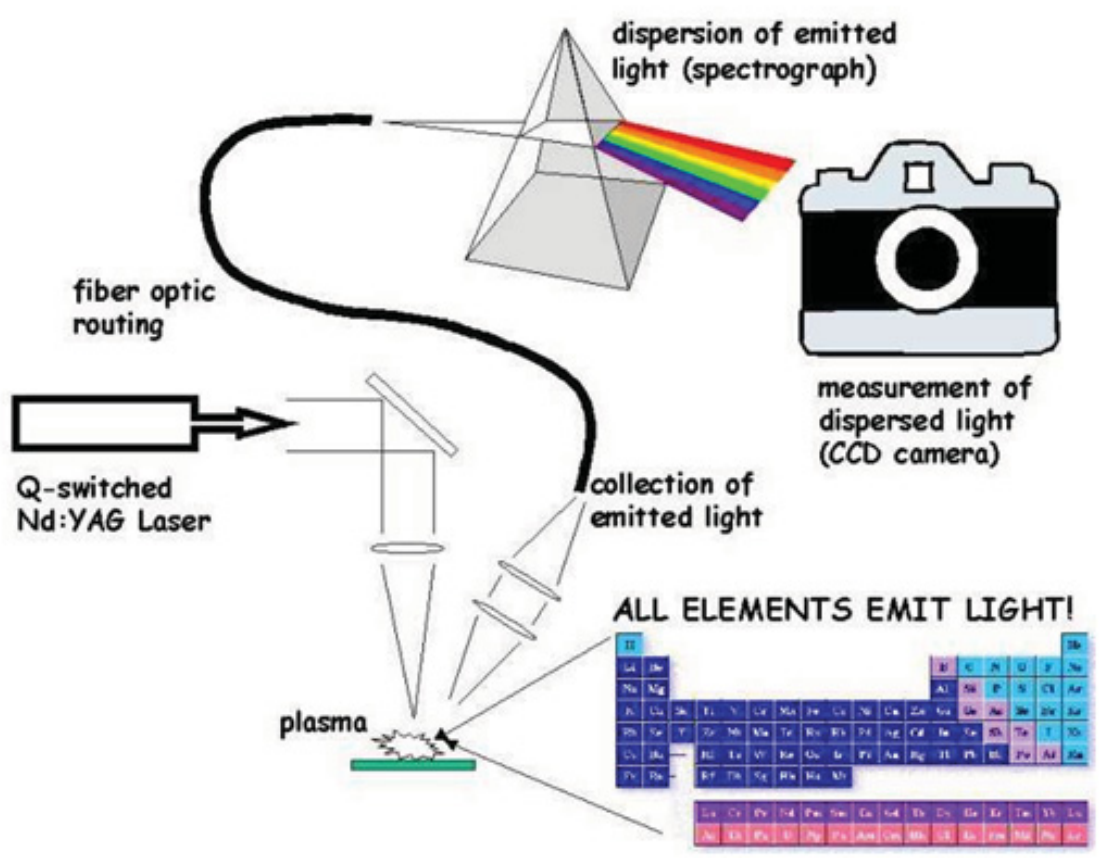

Figure 59. Schematic of a LIBS system - Courtesy of US Army Research Laboratory. ${ }^{141}$

Throughout the early plasma and extreme temperatures, the ablated materials break down into excited ionic and atomic species. Through this time, the plasma emits a continuum of radiation with no unique sig- 
nature about the species. As the plasma expands at supersonic velocities, it cools. Then, the characteristic atomic emission lines of the elements can be collected as shown in Figure 60. The time delay from the generation of continuum radiation to the characteristic radiation is approximately $10 \mu \mathrm{s}$; and thus, it requires the detector to be gated.

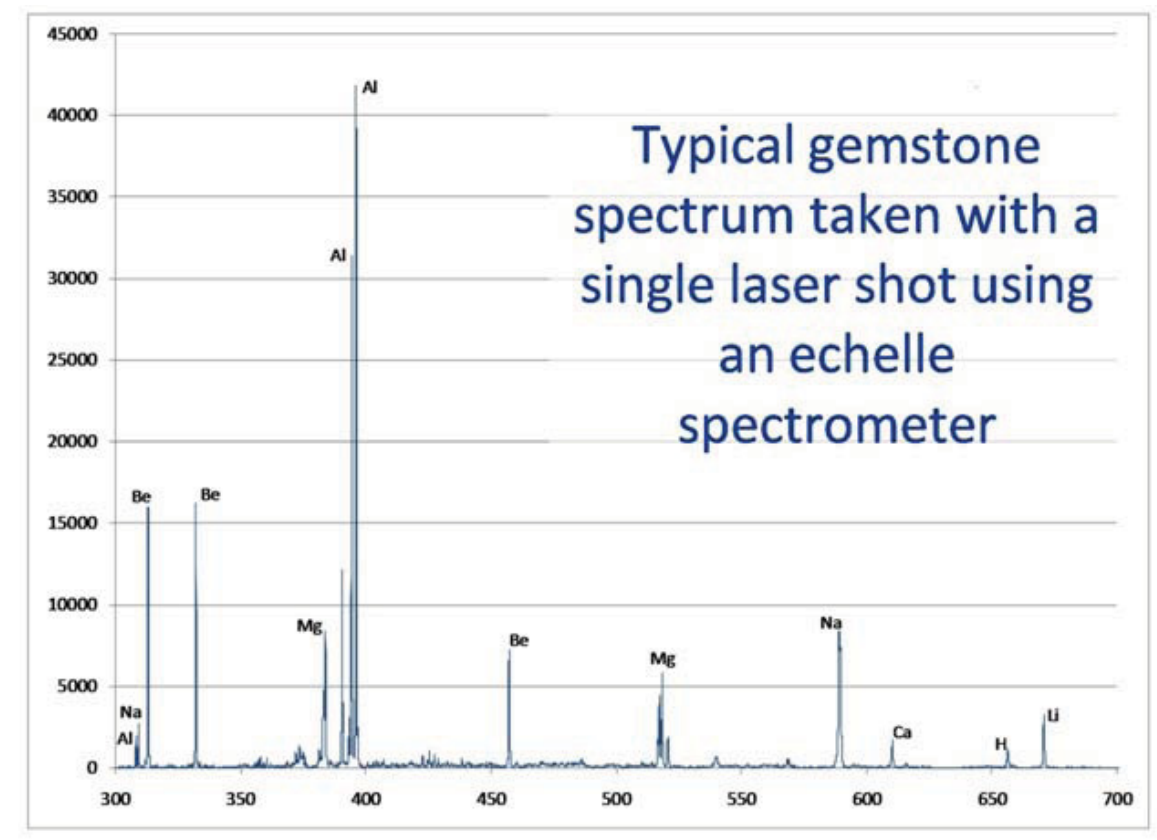

Figure 60. Broadband LIBS spectrum contains full information from $200-900 \mathrm{~nm}$. X-axis units are nm, and $\mathrm{Y}$-axis units are relative intensity. ${ }^{142}$

LIBS is related to a number of other laser-based analytical techniques such as vibrational spectroscopic technique of Raman spectroscopy, and fluorescence spectroscopic technique of laser-induced fluorescence. Instruments are now being sold that combine these techniques in a single instrument, enabling the atomic, molecular and structural characterization of a materials surface. Calibrations as shown in Figure 61 can be used to quantify concentrations; or chemometrics can be used to sort material.

Because a negligible amount of material is consumed during the LIBS process, the technique is minimally-destructive; and there is minimal specimen heating surrounding the ablation site. Preparation is often unnecessary where heterogeneity is to be investigated. One salient advantage of the LIBS technique is the capability to depth profile a specimen by repeatedly discharging the laser in the same position. As a layer is removed, the laser digs into the specimen with each shot. Surface contamination can also be removed in this manner. LIBS is a high through-put technique producing results within seconds enabling on-line industrial monitoring.

\subsubsection{Implementation into an Irradiation Test Capsule}

LIBS is an optical method requiring only line of sight optical access to the specimen and is non-invasive and non-contact. Fiber optics can be used for remote analyses. These attributes are useful for harsh hazardous environments and can characterize any material. The source laser can be rastered over the surface of the specimen creating spatially resolved elemental maps. The elemental maps can be used to determine concentration gradients, measure diffusion or composition ratios.

LIBS measurement reproducibility is limited by the stability of laser pulse energy and shape which affects the ensuing plasma. The elemental composition accuracy of LIBS measurements is normally better than 


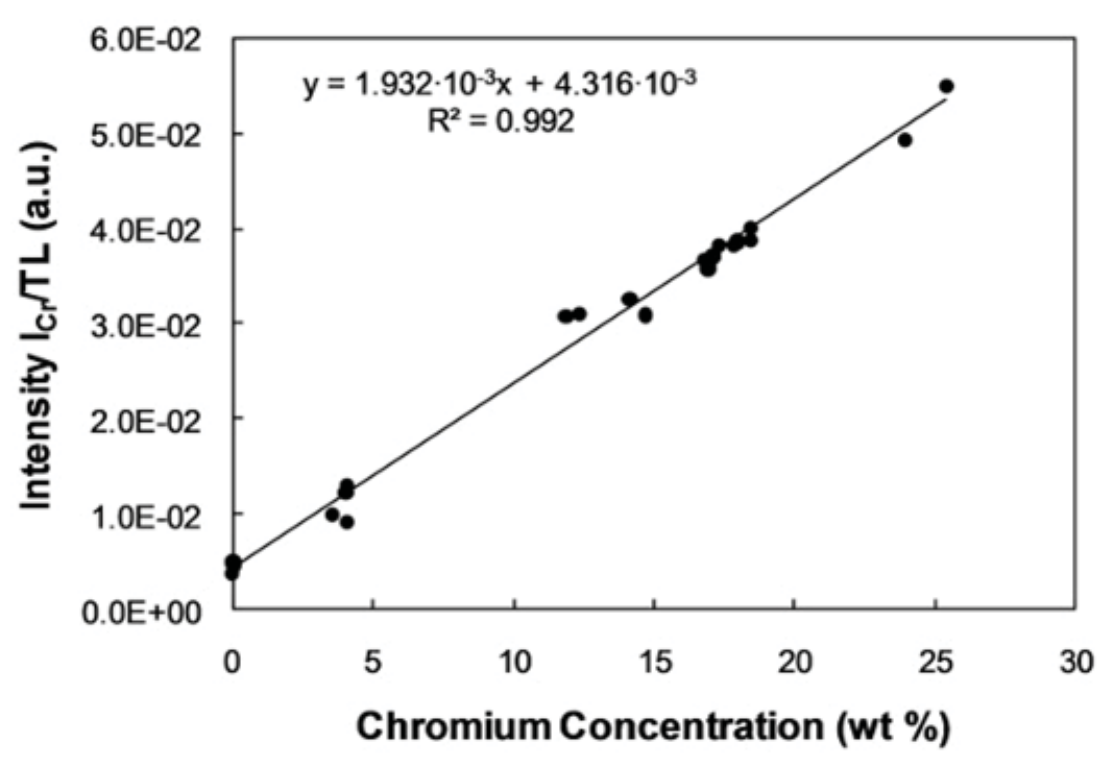

Figure 61. Chromium concentration in steel alloys of differing composition is shown. Calibrations can be used to quantify concentrations, or chemometrics can be used to sort material. ${ }^{142}$

$10 \%$. Detection limits of 1 to $30 \mathrm{ppm}$ by mass are typical but can range from $>100 \mathrm{ppm}$ to $<1 \mathrm{ppm}$ and are element dependant.

A classic LIBS system as shown in Figure 59, consists of a neodymium doped yttrium aluminum garnet (Nd:YAG) laser and a spectrometer with a fast response rate and time gated detector. The preferred spectrometer embodiment consists of a Czerny-Turner type to disperse the radiation onto a CCD line camera. This configuration allows for the simultaneous acquisition of the wavelength range of interest. The spectrometer response is normally from $1100 \mathrm{~nm}$ (near infrared) to $170 \mathrm{~nm}$ (deep ultraviolet) and is approximately the response range of the CCD detector. The emission lines from all elements are within this wavelength range. A computer is used to process and analyze the acquired data. LIBS is a simple spectroscopic analytical tool which makes it cost effective and easy to operate.

The configuration of a LIBS system shown in Figure 59 would not be convenient to use where space is limited such as within a nuclear reactor core. To make the LIBS system more compact and easier to implement in confined spaces, a double-clad fiber (DCF) can be used to deliver the excitation light and collect the atomic emission lines. The use of fiber optics to deliver the excitation light and return the signal spectrum allows for convenient sensing setups over long distances $(100 \mathrm{~m})$. As shown in Figure 62. a DCF is an optical fiber that has a central core and two layers of large-diameter cladding. The central core may be single-mode or may be multimode with a low numerical aperture. Typically, both cladding layers have lower refractive index than the core. The inner cladding has lower refractive index than the outer cladding. This enables the inner cladding to transmit multimode light with a different wavelength from that carried in the central core of the fiber.

Since a single fiber can carry both the excitation and signal light, the LIBS system can be more compact, easier to implement, and easier to use. A single lens can be designed to focus the generation light in the central core onto the surface while being able to collect into the inner cladding the diffuse signal light generated by the atomic emission lines. A double-clad fiber coupler ${ }^{148}$ can be used to get both beams into the fiber as well as to retrieve the signal light. The use of double clad fiber will allow for a compact sensor 


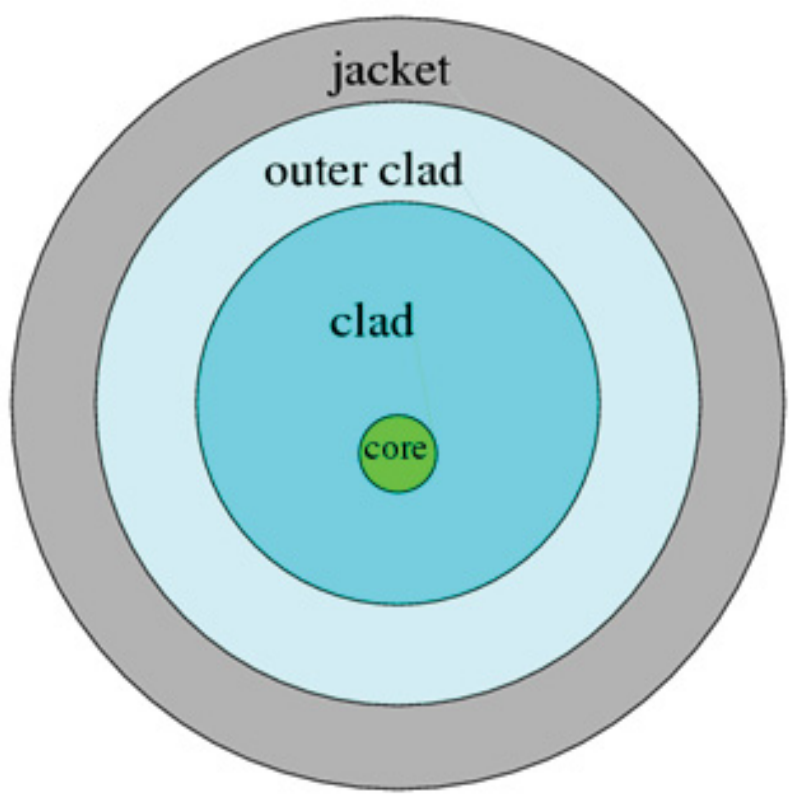

Figure 62. Cross-section of circular DCF with offset core.

head ( $\approx 5 \mathrm{~mm}$ diameter), ensure that the collection optics are always in alignment with the light generating plasma, and facilitate a robust system design.

\subsubsection{Current Status and Required R\&D for Deployment}

LIBS technology has been commercially applied to manufacturing production in a harsh galvanizing steel making environment. ${ }^{146}$ The technology will need to be adapted to in-pile applications that have constraints unique to the nuclear industry. Like all fiber-based systems, LIBS will be sensitive to attenuation caused by photo darkening of the fiber by the nuclear radiation. The LIBS technique does require line of site access to the specimen surface immersed in a gas or vacuum. LIBS will work with any material including metals and ceramics.

The fiber delivery system can penetrate both the pressure vessel with commercial feed-throughs or Conax fittings. Probably the most convenient way to get the sensor head to the top end of the in-pile material sample is to go though the end cap of the cladding via a fiber optic feed through.

\subsection{Electrical Conductivity}

Electrical conductivity measurements offer the opportunity to qualitatively detect real time dimensional changes in uranium dioxide $\left(\mathrm{UO}_{2}\right)$ fuel pellet structure (swelling and cracking) during irradiation. As discussed in this section, this technology could be implemented in an instrumented lead capsule located in an ATR irradiation position containing one or more uranium dioxide fuel pellets. Four conductors will be required in order to make a four point conductivity measurement. Two conductors will deliver excitation current to the ends of the fuel pellet. Two other conductors will measure the resulting potential drop across the pellet. Conductivity will be derived from the current and voltage relationships. The goal is not to make an accurate quantitative measurement of $\mathrm{UO}_{2}$ conductivity but to measure real time relative conductivity changes during neutron radiation. These changes are proposed to be related to pellet temperature, swelling, and cracking. 


\subsubsection{Description}

As discussed in Section 2, $\mathrm{UO}_{2}$ is commonly used as a fuel in existing LWRs and some fast reactors. It also has been reported to have very good semiconductor properties. ${ }^{149}$ This means that it has an electrical conductivity between that of a conductor and an insulator. This property will be exploited in the proposed sensor technology. The band gap energy of $\mathrm{UO}_{2}$ is between that of silicon $(\mathrm{Si})$ and gallium arsenide (GaAs) which are commonly used semiconductors today. $\mathrm{UO}_{2}$ also has other very interesting electrical properties that may be of value such as a high dielectric constant, a high Seebeck coefficient, and high solar cell efficiency.

Electrical conductivity is generally a function temperature. $\mathrm{UO}_{2}$ goes through greater than two orders of magnitude of conductivity change from ambient temperature to $925^{\circ} \mathrm{C} .{ }^{149}$ At one time, electrical resistors were made of $\mathrm{UO}_{2}$ due to their ability to operate at high temperatures. Hence, it should be possible to trend pellet temperature during normal operation. Conductivity also is a function of the pellet dimensions. It is expected that small cracks and porosity changes will to lead to a measurable conductivity change, and certainly large cracks will lead to detectable changes. This could lead to a way to measure the onset of fuel pellet structural changes during thermal transients.

Prior to any irradiation testing, laboratory testing will be required to demonstrate this proposed technology. Simulant fuel pellets, constructed of ceramic materials with similar electrical properties, will be instrumented and placed in an electrically heated furnace. Heat rates typical of normal and transient operation can be simulated over time to evaluate the experiment method.

\subsubsection{Desired Phenomena to Detect}

Examinations of fuel swelling, porosity changes, and cracking effects occur during post irradiation examination. As discussed in Section 4.2, in-pile thermal conductivity measurements can provide insights about these effects. This section proposes a new method for real time indication of the effect occurrence and to some degree the magnitude of the effect. During the irradiation, the following parameters will be inferred with this sensor:

- The fuel pellet temperature. This should be a fairly accurate measure prior to the onset of swelling, porosity, and cracking. The pellet temperature profile should match the startup power profile and should remain some what constant during steady state operation.

- Gradual electrical conductivity changes. While holding cooling temperature and reactor power constant, gradual conductivity changes are assumed to be primarily attributed to swelling and porosity changes. Changes due to swelling and porosity are assumed to be associated to a reduction in the area of the pellet diameter that can be utilized by electrons for current conduction. Secondary effects, such as the generation of conductive fission product gasses filling voids and solids changing the bulk conductivity, could also be detected.

- Instantaneous electrical conductivity changes. Instantaneous changes in conductivity could also be detected if they are due to instantaneous generation of cracks in the fuel pellet matrix.

As noted above, the primary benefit of this method over other probes that could be used to infer changes in fuel morphology is that it is non-intrusive. The hot wire method requires that the fuel be heated by an external heat source (a wire carrying a significant current) and that the fuel pellet be penetrated with a sensor. A second benefit is that the hundreds of samples per second could be taken profiling the growth of a crack. However, the use of this method requires prior knowledge of what phenomena (e.g., crack formation, swelling, etc.) are causing the observed change in electrical conductivity. 


\subsubsection{Required R\&D for Deployment}

The technology required to deploy this sensor currently exists. The R\&D required for deployment is to characterize whether the proposed technology will yield data of sufficient integrity that it can be used to infer changes in fuel morphology.

A conceptual design for an ATR or HFIR test capsule exploiting this design is shown in Figure 63. Initially, it is proposed that the feasibility of this technology be investigated using laboratory thermal cycle tests with a small inert gas filled capsule constructed of stainless steel or other reactor fuel pin cladding material, such as zircaloy. The capsule will contain one simulant fuel pellet, made of material with similar electrical properties as $\mathrm{UO}_{2}$. One is selected over more than one to avoid conductivity issues associated with pellet to pellet contact points. Conductivity measurements associated with a fuel pellet, not a stack of pellets, are proposed. Four compression loaded contacts are used to support a four point conductivity measurement. Contact material will be selected such that it can withstand fuel pellet temperatures that occur in operating reactors. The diameter of the capsule will be on the order of the diameter of a typical fuel pin (e.g., less than $2.54 \mathrm{~cm}$ ). The length will be about $15 \mathrm{~cm}$.

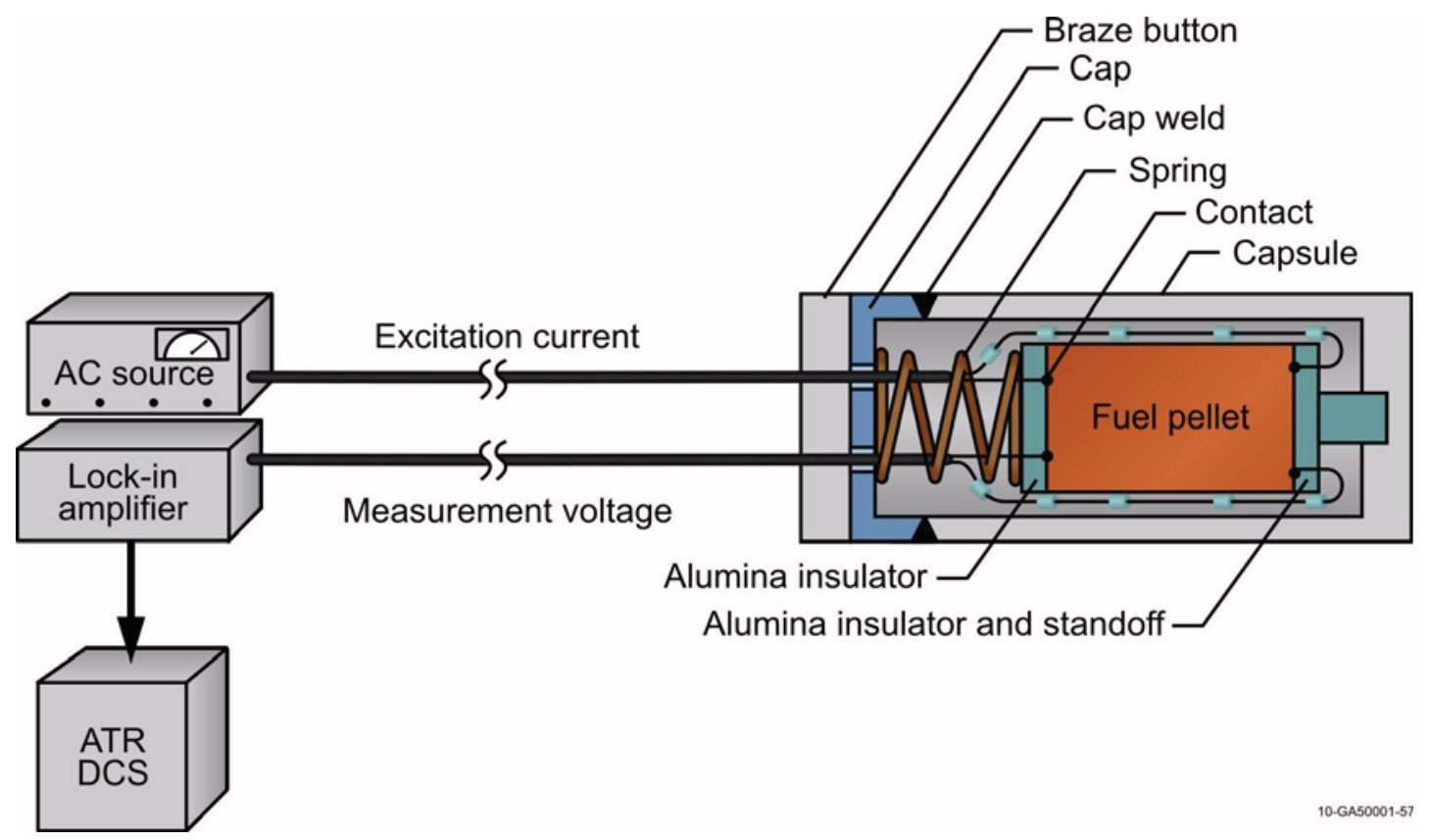

Figure 63. Conceptual design for laboratory test capsule.

Two of the contacts will be located at one end of the pellet and two at the other end. The contacts at both ends will be imbedded in an aluminum dioxide spacer. The spacers will be held in compression with the pellet by spring compression at one end and hard mounted to the capsule at the other end. The spring and hard mount will also hold the pellet away from the capsule wall to eliminate pellet to wall conductivity paths and define a space where two of the wires will be routed. The wires will be threaded through aluminum oxide beads to also prevent electrical and physical contact with the wall. Two hard sheathed cable pairs will be required to conduct the excitation in and the signal out of the reactor vessel. They will be brazed to the capsule top at one end. One pair (one at each end) will be used to excite an alternating current flow through the fuel pellet from a low impedance current source. The second set will be used to measure the current induced voltage drop across the pellet using a high impedance lock-in amplifier. The lock-in will be used to synchronously measure only the voltage associated with the current flow from the AC source and reject all other voltages as noise. The voltage drop is directly related to electrical conductivity. 
The capsule will be placed in an electrically heated furnace that is purged with inert gas. The furnace will slowly be brought from ambient temperature to a temperature based on fuel pellet temperatures of operating reactors. ${ }^{150}$ The experiment will be monitored for the duration of a typical operating cycle ( $\sim 40$ days). It is expected that the conductivity will follow the as-measured temperature/time profile.

Next, a series of temperature transients will be initiated to induce cracking in the simulant fuel (similar to the fuel pellet shown in Figure 8). The electrical conductivity profile should follow the temperature/time profile, but now super-imposed on the profile should be conductivity step functions due to the propagation of cracks in the fuel. After completion of the transient testing, the capsule will be opened; and visual examination will be completed.

Given successful completion of laboratory experiments, an instrumented lead capsule will be fabricated that contains a $\mathrm{UO}_{2}$ fuel pellet. The proposed sensor can be deployed in the ATR or HFIR using fabrication techniques similar to those that were successfully demonstrated for the hot wire needle probe (see Section 4.2). Connections and leads for supplying current and detecting voltage will be combined into two leads that connect to the AC source and amplifier that are located outside of the reactor vessel. Data will be acquired and stored by a data acquisition system. After the operating cycle is complete, the capsule will be opened; and a post irradiation visual examination would be performed for data comparison.

If the laboratory or irradiation tests were coupled with a hot wire thermal conductivity needle probe (see Section 4.2), then changes in thermal conductivity could be correlated with electrical conductivity.

\subsubsection{Desired Physical Parameters to be Detected}

As described in this section, a new candidate sensor technology is proposed that will allow the following to be measured with a non-intrusive sensor:

- Real-time non-intrusive fuel pellet bulk temperature of fuel early in burn up and prior to undergoing swelling, porosity changes, or cracking;

- Real time non-intrusive measurement of instantaneous crack growth occurrence;

- Real time non-intrusive measurement of porosity and swelling.

As noted in this section, this technology is only suited for ceramic fuel contained within a capsule containing inert gas. Although the technology currently exists for deploying this technology, laboratory evaluations are needed to estimate the accuracy of the proposed method.

\subsection{Summary}

Several candidate sensor technologies, not currently used for in-pile applications, are described in this section that could improve the fidelity of data obtained during fuel irradiations. Table 14 lists these technologies with applicable test conditions where they could be deployed, parameters that they could detect, and anticipated accuracies for these parameters. As indicated in this table, there is some uncertainty with respect to feasibility and accuracy for many of these candidate technologies. As part of the FCR\&D instrumentation development effort, modeling experts were asked to prioritize which parameters were of interest for various fuel types; and instrumentation experts were asked to rank the likelihood of the sensor technologies identified in this section and other instrumentation technologies to provide these parameters of interest with the desired accuracy. Results from this evaluation are discussed in Section 6. 
Table 14. Comparison of candidate instrumentation technologies

\begin{tabular}{|c|c|c|c|c|c|c|}
\hline \multirow{2}{*}{ Technology } & \multicolumn{2}{|c|}{ Test Conditions } & \multicolumn{2}{|c|}{ Possible Parameters } & \multicolumn{2}{|c|}{ Comments } \\
\hline & Fuel & Environment & Parameter & Resolution/Accuracy & Advantages & Limitations \\
\hline \multirow[t]{7}{*}{ Fiber Optics } & \multirow{7}{*}{$\begin{array}{l}\text { Ceramic } \\
\text { and Metal }\end{array}$} & \multirow{7}{*}{$\begin{array}{l}\text { Inert gas } \\
\text { (possibly } \\
\text { liquid metal } \\
\text { for some } \\
\text { applications } \\
\text { with } \\
\text { appropriate } \\
\text { coatings) }\end{array}$} & Temperature & Unknown & \multirow{2}{*}{$\begin{array}{l}\text { Small diameter; } \\
\text { non-contact, } \\
\text { distributed } \\
\text { sensor }\end{array}$} & \multirow{2}{*}{$\begin{array}{l}\text { Fiber darkening may } \\
\text { affect accuracy and } \\
\text { longevity }\end{array}$} \\
\hline & & & Pressure & Unknown & & \\
\hline & & & Strain & Unknown & \multirow{4}{*}{$\begin{array}{l}\text { Small diameter; } \\
\text { distributed } \\
\text { sensor }\end{array}$} & \multirow{4}{*}{$\begin{array}{l}\text { Indirect measurement } \\
\text { based on fiber } \\
\text { properties }\end{array}$} \\
\hline & & & Elastic Properties & Unknown & & \\
\hline & & & $\begin{array}{l}\text { Crack Initiation } \\
\text { and Growth }\end{array}$ & Unknown & & \\
\hline & & & Porosity & Unknown & & \\
\hline & & & $\begin{array}{l}\text { Thermal } \\
\text { conductivity }\end{array}$ & Unknown & Unknown & Unknown \\
\hline \multirow[t]{9}{*}{ Ultrasonics } & \multirow[t]{9}{*}{$\begin{array}{l}\text { Ceramic } \\
\text { and Metal }\end{array}$} & \multirow[t]{9}{*}{$\begin{array}{l}\text { Inert gas and } \\
\text { liquid metal }\end{array}$} & $\begin{array}{l}\text { Temperature (see } \\
\text { Section 4.1) }\end{array}$ & $2 \%$ & $\begin{array}{l}\text { Small diameter, } \\
\text { distributed } \\
\text { sensor }\end{array}$ & $\begin{array}{l}\text { Demonstrated up to } \\
3000^{\circ} \mathrm{C}\end{array}$ \\
\hline & & & $\begin{array}{l}\text { Thermal } \\
\text { conductivy/ } \\
\text { thermal diffusivity }\end{array}$ & Unknown & $\begin{array}{l}\text { Small diameter, } \\
\text { distributed } \\
\text { sensor }\end{array}$ & $\begin{array}{l}\text { Accuracy dependent on } \\
\text { sample material and } \\
\text { temperature }\end{array}$ \\
\hline & & & Length & $1 \mu \mathrm{m}^{\mathrm{a}}$ & \multirow{7}{*}{$\begin{array}{l}\text { Non-intrusive } \\
\text { sensor }\end{array}$} & \multirow{7}{*}{$\begin{array}{l}\text { Higher accuracies } \\
\text { possible with } \\
\text { magnetostrictive or } \\
\text { piezoelectric } \\
\text { transducers }\end{array}$} \\
\hline & & & $\begin{array}{l}\text { Diameter } \\
\text { (swelling, } \\
\text { corrosion) }\end{array}$ & $0.05 \mu \mathrm{m}^{\mathrm{a}}$ & & \\
\hline & & & $\begin{array}{l}\text { Crack Initiation } \\
\text { and Growth }\end{array}$ & $1 \mu \mathrm{m}^{\mathrm{a}}$ & & \\
\hline & & & Density & $\sim 1 \%$ & & \\
\hline & & & Young's Modulus & $\sim 1 \%$ & & \\
\hline & & & Porosity & Shape dependent, $1-25 \%$ & & \\
\hline & & & $\begin{array}{l}\text { Grain size / } \\
\text { Microstructure }\end{array}$ & Unknown $^{b}$ & & \\
\hline \multirow[t]{2}{*}{$\begin{array}{l}\text { Laser } \\
\text { Ultrasound }\end{array}$} & \multirow[t]{2}{*}{ Metal } & \multirow[t]{2}{*}{ Inert gas } & $\begin{array}{l}\text { Porosity and } \\
\text { microstructure } \\
\text { inferred from } \\
\text { acoustic and } \\
\text { resonant analysis }\end{array}$ & Unknown & \multirow[t]{2}{*}{$\begin{array}{l}\text { Non-contact } \\
\text { sensor }\end{array}$} & \multirow[t]{2}{*}{$\begin{array}{l}\text { Fiber darkening may } \\
\text { affect longevity }\end{array}$} \\
\hline & & & \begin{tabular}{|l} 
Mechanical \\
properties inferred \\
from acoustic and \\
resonant analysis
\end{tabular} & Unknown & & \\
\hline \multirow[t]{2}{*}{ Borescope } & \multirow[t]{2}{*}{$\begin{array}{l}\text { Ceramic } \\
\text { and Metal }\end{array}$} & \multirow[t]{2}{*}{ Inert gas } & $\begin{array}{l}\text { Crack initiation } \\
\text { and growth }\end{array}$ & $\sim 100 \mu \mathrm{m}$ & \multirow{2}{*}{$\begin{array}{l}\text { Small diameter, } \\
\text { non-contact } \\
\text { sensor with } \\
\text { visual image }\end{array}$} & \multirow[t]{2}{*}{$\begin{array}{l}\text { Fiber darkening may } \\
\text { affect longevity }\end{array}$} \\
\hline & & & Porosity & $\sim 100 \mu \mathrm{m}$ & & \\
\hline \multirow{2}{*}{$\begin{array}{l}\text { Laser-induce } \\
\text { d Breakdown } \\
\text { Spectroscopy }\end{array}$} & \multirow[t]{2}{*}{$\begin{array}{l}\text { Ceramic } \\
\text { and Metal }\end{array}$} & \multirow[t]{2}{*}{ Inert gas } & $\begin{array}{l}\text { Elemental } \\
\text { composition }\end{array}$ & $5-10 \%$ & \multirow[t]{2}{*}{$\begin{array}{l}\text { Non-contact } \\
\text { sensor }\end{array}$} & \multirow[t]{2}{*}{$\begin{array}{l}\text { Fiber darkening may } \\
\text { affect signal/noise ratio }\end{array}$} \\
\hline & & & $\begin{array}{l}\text { Microstructure } \\
\text { changes inferred } \\
\text { from composition }\end{array}$ & Unknown & & \\
\hline $\begin{array}{l}\text { Electrical } \\
\text { Conductivity }\end{array}$ & $\begin{array}{l}\text { Ceramic } \\
\left(\mathrm{UO}_{2}\right)\end{array}$ & Inert gas & $\begin{array}{l}\text { Crack initiation } \\
\text { and growth } \\
\text { Porosity and } \\
\text { swelling } \\
\text { Temperature }\end{array}$ & $\begin{array}{l}\text { Four point resistivity } \\
\text { measurement accurate to } \\
<1 \% \text {; accuracy unknown } \\
\text { for relating resistivity to } \\
\text { other parameters }\end{array}$ & $\begin{array}{l}\text { Non-intrusive } \\
\text { sensor; no } \\
\text { technology } \\
\text { development } \\
\text { required }\end{array}$ & $\begin{array}{l}\text { Non-intrusive sensor; } \\
\text { tests needed to quantify } \\
\text { validity }\end{array}$ \\
\hline
\end{tabular}

a. Reported accuracies depending on sample material and geometry and test conditions.

b. Changes in grain sized inferred from signal. 


\section{SUMMARY AND RECOMMENDATIONS}

New and enhanced nuclear fuels are a key enabler for new and improved reactor technologies. For example, the goals of the NGNP will not be met without irradiations successfully demonstrating the safety and reliability of new fuels. Likewise, fuel reliability has become paramount in ensuring the competitiveness of nuclear power plants. Recently, the DOE-NE launched a new direction in fuel research and development that emphasizes an approach relying on first principle models to develop optimized fuel designs that offer significant improvements over current fuels. To facilitate this approach, high fidelity, real-time, data are essential for characterizing the performance of new fuels during irradiation testing. A three-year strategic research program is proposed for developing the required test vehicles with sensors of unprecedented accuracy and resolution for obtaining the data needed to characterize three-dimensional changes in fuel microstructure during irradiation testing. When implemented, this strategy will yield test capsule designs for the ATR and the HFIR that are instrumented with new sensor technologies and other possible irradiation locations considered for the FCR\&D program. Prior laboratory testing and, as needed, irradiation testing, of these sensors will have been completed to give sufficient confidence that the irradiation tests will yield the required data.

From the onset of this instrumentation development effort, it was recognized that obtaining these sensors must draw upon the expertise of a wide-range of organizations not currently supporting nuclear fuels research. Hence, a draft version of this document was developed to provide necessary background information related to fuel irradiation testing, desired parameters for detection, and an overview of currently available in-pile instrumentation. Then, a workshop was held in which U.S. and foreign experts from fuels, irradiation, and instrumentation fields participated. Prior to this workshop, draft versions of this document were distributed to participants to stimulate expert interactions at this meeting. During the workshop, candidate sensor technologies identified in this document were discussed and ranked by the experts using agreed upon criteria. The final version of this document describes the consensus reached during the workshop with respect to the path forward for accomplishing the goals of this research program.

\subsection{Test Conditions and Current In-Pile Instrumentation}

\subsubsection{Test Conditions and Desired Data}

Based on information provided in Section 2, it was agreed that instrumentation development efforts should focus on three types of fuel irradiation conditions: ceramic fuel surrounded by helium gas for LWRs; ceramic fuel surrounded by helium gas for SFRs; and metallic fuel surrounded by molten sodium for SFRs. Typical test conditions and geometries for each case are provided in Section 2. Although it is recognized that some tests could be performed in non-prototypic conditions (e.g., metallic fuel surrounded by inert gas), experts at the FCR\&D workshop noted that such tests must be carefully planned to demonstrate their applicability. Hence, experts recommended that initial efforts in this instrumentation development program emphasize sensors that can provide data in prototypic conditions.

Initial discussions with fuel performance modeling experts indicate that the parameters listed in Table 15 should be measured with the desired accuracies and spatial resolution. Discussions with fuel modelers indicate that detection of thermal properties is of highest priority, followed by fuel internal pressure due to fission gas release, swelling, crack growth, and constituent migration. However, as noted in Section 2, the FCR\&D program is placing the highest priority on sensors that can provide insights related to changes in fuel morphology because such insights are needed to clarify changes in other parameters, such as thermal conductivity, density, etc. 
Table 15. Summary of desired parameters for detection during fuel irradiation tests.

\begin{tabular}{|c|c|c|c|}
\hline \multirow{2}{*}{ Parameter } & \multirow{2}{*}{ Representative - Peak Value } & \multicolumn{2}{|c|}{ Desired } \\
\hline & & Accuracy & Spatial Resolution \\
\hline \multirow[t]{3}{*}{ fuel temperature } & Ceramic LWR - $1400^{\circ} \mathrm{C}$ & \multirow[t]{3}{*}{$2 \%$} & \multirow{3}{*}{$\begin{array}{l}1-2 \mathrm{~cm} \text { (axially); } \\
0.5 \mathrm{~cm} \text { (radially) }\end{array}$} \\
\hline & Ceramic SFR- $2600{ }^{\circ} \mathrm{C}$ & & \\
\hline & Metallic SFR $-1100^{\circ} \mathrm{C}$ & & \\
\hline \multirow[t]{3}{*}{ cladding temperature } & Ceramic LWR $<400{ }^{\circ} \mathrm{C}$ & \multirow[t]{3}{*}{$2 \%$} & \multirow[t]{3}{*}{$1-2 \mathrm{~cm}$ (axially) } \\
\hline & Ceramic SFR $-650^{\circ} \mathrm{C}$ & & \\
\hline & Metallic SFR - $650^{\circ} \mathrm{C}$ & & \\
\hline \multirow[t]{3}{*}{ pressure in fuel rod plenum } & Ceramic LWR - 800 psi & $5 \%$ & \multirow[t]{3}{*}{$\mathrm{NA}^{\mathrm{a}}$} \\
\hline & Ceramic SFR-1250 psi & $5 \%$ & \\
\hline & Metallic SFR - $1250 \mathrm{psi}$ & $5 \%$ & \\
\hline $\begin{array}{l}\text { fission gas release (amount } \\
\text { and composition) }\end{array}$ & $0-100 \%$ of inventory & $10 \%$ & NA \\
\hline \multirow{3}{*}{$\begin{array}{l}\text { fuel and cladding } \\
\text { dimensions radial and axial } \\
\text { (includes fuel-cladding gap } \\
\text { size) }\end{array}$} & Initial Length, $1 \mathrm{~cm}$ & $1 \%$ & NA \\
\hline & Outer diameter/Strain, $0.5 \mathrm{~cm} / 5-10 \%$ & $0.1 \%$ & NA \\
\hline & Fuel-Cladding Gap (0-0.1 mm) & $0.1 \%$ & NA \\
\hline \multirow{3}{*}{$\begin{array}{l}\text { fuel morphology/ } \\
\text { microstructure/cracking/ } \\
\text { constituent migration }\end{array}$} & Grain size, $10 \mu \mathrm{m}$ & $5 \%$ & $1-10 \mu \mathrm{m}$ \\
\hline & Swelling/Porosity, 5-20\% & $2 \%$ & \\
\hline & Crack formation and growth & $2 \%$ & $10-100 \mu \mathrm{m}$ \\
\hline \multirow[t]{2}{*}{ fuel thermal properties } & $\begin{array}{c}\text { Thermal conductivity, } \\
\text { Ceramic: }<5 \mathrm{~W} / \mathrm{mK} ; \text { Metallic: }<50 \mathrm{~W} / \mathrm{mK}\end{array}$ & $4 \%$ & $<1 \mathrm{~cm}$ (radially) \\
\hline & $\begin{array}{l}\text { Density (estimated from changes in length, } \\
\text { diameter, porosity, etc.) }\end{array}$ & $2 \%$ & NA \\
\hline \multirow{2}{*}{$\begin{array}{l}\text { neutron flux for estimating } \\
\text { fluence and fuel burnup }\end{array}$} & Thermal neutron flux $-\sim 1-5 \times 10^{14} \mathrm{n} / \mathrm{cm}^{2}-\mathrm{s}$ & $1-10 \%$ & $5 \mathrm{~cm}$ (axially) \\
\hline & $\begin{array}{l}\text { Fast neutron flux }(\mathrm{E}>1 \mathrm{MeV})-\sim 1-5 \times 10^{14} \\
\mathrm{n} / \mathrm{cm}^{2}-\mathrm{s}\end{array}$ & $15 \%$ & $5 \mathrm{~cm}$ (axially) \\
\hline
\end{tabular}

a. NA-Not Applicable.

\subsubsection{Currently Available Instrumentation to Provide Required Data}

As discussed in Section 4, sensors that are currently available at other MTRs (see Table 16) can be used in FCR\&D irradiations to obtain many of the desired parameters with the accuracies specified in Table 15. However, it should be noted that many of the sensors used at other MTRs may need some enhancements before they can be successfully implemented in the higher flux, harsher test conditions typical of ATR and HFIR tests. If enhanced, these sensors can provide insights with respect to parameters, such as temperature, thermal conductivity, crack growth, and flux. However, in general, the resolution available with such sensors is limited due to the limited size of the irradiation test and the desire to minimize the impact of the sensor on test results. It should also be noted that existing and near-term sensor technologies do not provide any capability for detecting changes in fuel microstructure or constituent migration. 
Table 16. Summary of sensors deployed at MTRs.

\begin{tabular}{|c|c|c|c|}
\hline Parameter & Sensor & Operating Conditions & Accuracy \\
\hline \multirow[t]{6}{*}{ Temperature } & Melt Wires & $100-1200^{\circ} \mathrm{C}$ & $\begin{array}{l}2-3{ }^{\circ} \mathrm{C} \text {, but limited by } \\
\text { number of wires with } \\
\text { different melting points } \\
\text { included in test. }\end{array}$ \\
\hline & SiC monitors & $100-800^{\circ} \mathrm{C}$ & $2 \%$ \\
\hline & Thermocouples (N, K) & $100-1000^{\circ} \mathrm{C}$ & $2 \%$ \\
\hline & $\begin{array}{l}\text { Thermocouples } \\
\text { (HTIR-TCs) }\end{array}$ & $100-1800^{\circ} \mathrm{C}$ & $2 \%$ \\
\hline & $\begin{array}{c}\text { Thermocouples - Type C, } \\
\text { D, R, and S }\end{array}$ & $100-2000^{\circ} \mathrm{C}$ & $\begin{array}{l}2 \% \text { or worse, } \\
\text { decalibrate due to } \\
\text { transmutation }\end{array}$ \\
\hline & Ultrasonic Thermometers $^{\mathrm{a}}$ & $1300-3000{ }^{\circ} \mathrm{C}$ & $2 \%$ \\
\hline \multirow[t]{2}{*}{ Thermal Conductivity } & Multiple Thermocouple & $\begin{array}{l}100-2000{ }^{\circ} \mathrm{C} \text {, depending on } \\
\text { thermocouple type }\end{array}$ & $2-8 \%$ \\
\hline & Hot Wire Needle Probe & $\begin{array}{c}100-2000{ }^{\circ} \mathrm{C}, \text { depending on } \\
\text { materials selected }\end{array}$ & $2 \%$ \\
\hline \multirow[t]{2}{*}{ Density / Geometry Changes } & Length - LVDT & up to $500^{\circ} \mathrm{C}^{\mathrm{b}}$ & $1-10 \mu \mathrm{m}$ \\
\hline & Diameter - Diameter Gauge & up to $500^{\circ} \mathrm{C}^{\mathrm{b}}$ & $1-10 \mu \mathrm{m}$ \\
\hline Crack Initiation /Growth & DCPD Method & $350^{\circ} \mathrm{C} / 2250 \mathrm{psia}$ & $\sim 20 \%$ \\
\hline Young's Modulus & Loaded Creep Specimen & up to $500^{\circ} \mathrm{C}^{\mathrm{b}}$ & $\sim 10 \%$ \\
\hline \multirow[t]{2}{*}{ Fission Gas/Pressure } & Sampling & Numerous isotopes & Unknown \\
\hline & Pressure gauge & $\begin{array}{c}15 \text { bar (220 psi) to } 70 \text { bar (1020 } \\
\text { psi) }\end{array}$ & $\begin{array}{l} \pm 0.2 \text { bar }(2.9 \mathrm{psi}) \text { to } \\
\pm 0.5 \text { bar }(7.3 \mathrm{psi})\end{array}$ \\
\hline \multirow[t]{3}{*}{ Flux - Thermal } & Flux wires / Foils & Material dependent & $\sim 10 \%$ \\
\hline & SPNDs & Dependent on emitter & $\sim 1-10 \%{ }^{\mathrm{c}}$ \\
\hline & Fission Chambers & Dependent on fissile deposit & $\sim 1-10 \%{ }^{\mathrm{c}}$ \\
\hline \multirow[t]{2}{*}{ Fast } & Flux wires / Foils & Material dependent & $\sim 1-10 \%$ \\
\hline & Fission Chambers & Dependent on fissile deposit & $\sim 1-10 \%$ \\
\hline
\end{tabular}

a. Prior in-pile use typically limited to short duration, fuel damage tests.

b. Some loss of accuracy at $350{ }^{\circ} \mathrm{C}$ due to Curie temperature effects, unless developmental IFE/HRP LVDTs are deployed.

c. Accuracy decreases with use.

\subsection{Expert Workshop Recommendations}

As part of the process to define the FCR\&D in- pile instrumentation development program, a workshop was help on November 10, 2010 in Las Vegas, Nevada, in which approximately forty international experts from fuels, irradiation, and instrumentation fields participated. During the workshop, candidate sensor technologies were discussed and ranked using agreed upon criteria. This section highlights the consensus reached with respect to the path forward for accomplishing the goals of this research program. More detailed information about the topics discussed at the workshop and the process used to gain a consensus may be found in Appendix A. 


\subsubsection{Expert Workshop Process}

During the workshop, experts discussed possible criteria and agreed to rank candidate technologies using the following five items:

- Potential to detect parameters with desired accuracy. A noted objective of the FCR\&D effort is to develop instrumentation capable of unprecedented accuracy and resolution for obtaining the data needed to characterize three-dimensional changes in fuel microstructure during irradiation testing. Many of the candidate technologies offer the potential for improved detection of the 'higher priority' parameters (e.g., thermal properties, cracking, porosity, etc.) identified by fuel modeling experts. However, experts agreed that there were limited sensor technologies offering the potential to directly detect changes in fuel microstructure during irradiation testing, which is the highest priority in the FCR\&D program.

- Potential to detect desired parameters in prototypic conditions (environment, temperature, etc.). Although experts recognized that some tests could be performed in non-prototypic conditions (e.g., metallic fuel surrounded by inert gas), it was agreed that such tests must be carefully planned demonstrate their applicability. Hence, sensors that have the potential to provide data in prototypic conditions were ranked more highly.

- Versatility. Section 2 identifies several parameters desired by fuel modeling experts. However, the size of irradiation tests limits the number of sensors that can be installed in a single test. Hence, experts viewed a single probe that can be used to detect a parameter at multiple locations more favorably. Likewise, from a funding perspective, a single technology that can provide data for detecting multiple parameters was ranked more highly because it offers the FCR\&D effort more opportunities.

- Ease of Installation. As documented in Sections 2 and 3, test rigs are complicated. The ease with which a sensor can be installed is another important consideration. Clearly, sensors are more desirable (and ranked more highly) if they can be installed without concerns about bends, breakage, special connectors, or a line of sight.

- Technology Readiness (demonstrated in-pile experience to obtained desired accuracy under desired conditions). Funding resources for the FCR\&D instrumentation development effort are limited. Technologies are less desirable if they require large investments to overcome large technology hurdles prior to deployment. Although experts deemed that all of the technologies described in Section 5 would require some investment to assess their viability for in-pile testing, experts favored technologies that could be deployed with less investment.

For this assessment, experts ranked the above criteria using the following numeric scores:

1 - Technology unable to meet most aspects of the criteria;

2 - Technology may offer potential to meet some aspects of the criteria;

3 - Technology may offer potential to meet most aspects of this criteria and some data available.

Experts agreed that a simpler ranking system was appropriate due to the availability of information related to technologies with respect to various criteria.

\subsubsection{Workshop Ranking Results and Discussion}

Table 17 summarizes the composite scores for the technologies ranked by experts at this workshop. Note that only a limited number of sensor technologies identified in Section 5 were ranked by the experts. Due to workshop time constraints, a preliminary ranking was completed by INL experts prior to the workshop 
(using the same criteria ultimately selected by the experts attending this workshop), and only those technologies that were ranked more highly were presented to and ranked by the experts.

Table 17. Composite scores from expert evaluations

\begin{tabular}{|l|c|}
\hline \multicolumn{1}{|c|}{ Technology } & Average Rank \\
\hline Fiber Optics & 11.2 \\
\hline Ultrasonic Techniques & 13.7 \\
\hline Laser Ultrasound & 10.5 \\
\hline $\begin{array}{l}\text { Time Reversal (TR) and Nonlinear Elastic Wave Spectroscopy (NEWS) for In-Situ } \\
\text { Material Integrity Diagnostics }\end{array}$ & 8.1 \\
\hline Optical Probes, Contact Stress Sensors, Photo-Acoustic Gas Sensor & 5.6 \\
\hline
\end{tabular}

Discussion prior to the ranking process (and the scores reported in Table 17) indicate that experts favored more mature technologies. Experts believed that existing research demonstrates that ultrasonic and fiber optics technologies offer the potential for some quick successes. For example, prior use of ultrasonic thermometers suggest that a single probe could be used to obtain a temperature profile with accuracies and resolutions not possible with existing technologies.

In addition, experts favored technologies that could obtain the desired data under prototypic conditions and if they offered the potential to detect most, if not all, of the parameters requested by fuel modeling experts. For example, ultrasonic sensors were ranked higher because of their potential to detect desired parameters in metallic fuel surrounded by sodium and in oxide fuel surrounded by helium for the range of temperatures of interest.

Experts also ranked technologies more highly if they offered the potential for 'diverse' parameter detection. For example, initial research investments in ultrasonic and fiber optic technologies could lead to methods that could ultimately be used to detect a wide range of parameters (e.g., physical and environmental parameters). In addition, investments in ultrasonics and fiber optic technologies offer the potential for later achieving more advanced technologies, such as laser ultrasonics, that require these technologies for successful detection and/or transmission.

Experts emphasized the need for U.S. researchers to work with organizations already possessing expertise in selected technologies. Where possible, the FCR\&D program should collaborate and/or learn from developments made by international nuclear instrumentation researchers and non-nuclear instrumentation researchers. In addition, any sensor in-pile deployment efforts should be coupled with post-irradiation examinations to maximize the insights obtained from any irradiation testing.

Experts also emphasized that sensor development efforts should simultaneously focus on capsule design and sensor viability tasks. Experts noted that having sensors installed and functioning in a viable test capsule was as important as development of the sensor. Prior to starting any research, experts emphasized the need for developing detailed plans with 'off-ramps' at key decision points where the viability of the technology could be assessed prior to further research.

Last, experts emphasized the need for U.S. MTRs to gain access to instrumentation available to users at foreign test reactors and to work toward improving the accuracy of existing sensors for detecting phenomena. It was noted that many of the sensors identified in Section 4 are not currently available for use in the higher flux, harsher test conditions proposed for FCR\&D irradiations. In addition, experts noted that additional research was needed to reduce uncertainties associated with existing sensors, such as those used for flux detection. 


\subsection{Summary and Recommendations}

A three-year strategic research program is proposed for developing the required test vehicles with sensors of unprecedented accuracy and resolution for obtaining the data needed to characterize three-dimensional changes in fuel microstructure during irradiation testing. This document defines the strategic program proposed for this effort. It provides the necessary background information related to fuel irradiation testing, desired parameters for detection, and an overview of currently available in-pile instrumentation. At the onset of this effort, it was recognized that this program must draw upon input from a wide-range of experts not currently supporting nuclear fuels research. Hence, a workshop was held to obtain this input and gain consensus on the approach recommended in this document. This document also includes recommendations from this workshop.

Based on the activities completed to develop this strategic plan, it is recommended that the FCR\&D instrumentation development program be initiated as a three year program that includes the following three tasks:

- Ultrasonics-Based Evaluations - In this task, laboratory evaluations and necessary irradiations will be completed to demonstrate the viability of this technology for in-pile applications. Specifically, tasks should be identified for obtaining the required signal processing upgrades, demonstrating the viability of ultrasonics to detect the parameters of interest under various anticipated test conditions (e.g., inert gas and sodium) with the required accuracy, and for developing test capsule(s) in which the proposed sensors could be deployed at the ATR and HFIR.

- Fiber Optics-Based Evaluations - In this task, laboratory evaluations and necessary irradiations will be completed to demonstrate the viability of this technology for in-pile applications. Specifically, tasks should be identified for obtaining the required signal processing, demonstrating the viability of fiber optics to detect the parameters of interest under various anticipated test conditions (e.g., inert gas and sodium) with the required accuracy (note that suitable coatings/sheaths must be identified for sodium applications and temperature limitations must be quantified). In addition, test capsule(s) designs should be developed in which the proposed sensors could be deployed at the ATR and HFIR.

- Upgrade US MTR In-Pile Instrumentation - In this task, laboratory evaluations and necessary irradiations will be completed to demonstrate the viability of enhanced detectors under anticipated ATR and HFIR irradiation conditions. Candidate sensors for enhancement include LVDT-based sensors for elongation and diameter evaluations, flux detectors, and thermal conductivity probes.

For each task, detailed project plans will be developed with a schedule identifying what can be accomplished within a three-year time period. Plans will also identify 'off-ramps' at key decision points where the viability of the technology could be assessed prior to further research. In addition, project plans will identify collaborations to ensure that research will take advantage of existing expertise for each technology. 


\section{REFERENCES}

1. P.Miller, "DOE Nuclear Energy Programs: The Future of Nuclear Energy - Part 1," December 18, 2009, presentation to Nuclear Energy Advisory Committee, Washington, DC.

2. P. Lyons, "DOE Nuclear Energy Programs: The Future of Nuclear Energy - Part 2," December 18, 2009, presentation to Nuclear Energy Advisory Committee, Washington, DC.

3. K.O. Pasamehmetoglu, "Fuel Cycle Research and Development Fuel Development Status," presentation to ANTT Meeting, February 2-3, 2010, Washington, DC.

4. D. C. Crawford, et al., "An approach to fuel development and qualification," Journal of Nuclear Materials 371 (2007) 232-242.

5. J. L. Rempe, D. L. Knudson, K. G. Condie, J. E. Daw, H. Ban, B. S. Fox, and G. E. Kohse, "New Sensors for the Advanced Test Reactor National Scientific User Facility" invited paper for ANIMMA Special Edition, IEEE Transactions on Nuclear Science, 57, No.5, pp 2653-2661, October 2010.

6. J-F. Villard, "State-of-the-art and Improvement of Online Measurements in Present and Future French Research Reactors," Seminar at the Idaho National Laboratory, September 16, 2010.

7. J-F Villard and M. Schyns, "Advanced In-Pile Measurements of Fast Flux, Dimensions, and Fission Gas Release," Proceedings of the ANS NPIC HMIT 2009 Topical Meeting on Nuclear Plant Instrumentation, Controls, and Human Machine Interface Technology, Knoxville, TN, April 2009.

8. K. N. Choo, B. G. Kim, M. S. Cho, Y. K.Kim, and J. J. Ha, "Measurement and Evaluation of the Irradiation Test Parameters for a Specimen in a HANARO Material Irradiation Capsule," Proceedings of the First International Conference on Advancements in Nuclear Instrumentation, Measurement Methods and their Applications (ANIMMA), Marseille, France, June 2009.

9. “JMTR Instrumentation Handbook," presented to J. Rempe during meetings at JMTR, Tokai, Japan, October 2009.

10. K. Bakker, "HFR Instrumentation," informal papers presented to J. Rempe and G. Kohse during meetings, Petten, Netherlands, October 2008.

11. S. Solstad \& R. Van Nieuwenhove, "Instrument Capabilities and Developments," Proceedings of the ANS 2009 Topical Meeting on Nuclear Plant Instrumentation, Controls, and Human Machine Interface Technology, Knoxville, TN, April 2009.

12. K. Maeda, S. Sasaki, M. Kato, Y. Kihara. J. Nucl. Mater. 389 (2009) 78.

13. P. Millet, INL, informal communication to J. Rempe, INL, June 2010.

14. N. Chauvin, A. Courcelle, M. Pelletier, Y. Guerin, JM. Escleine, M. Phelip, F. Michel, S. Bejaoui, and M. Lainet, "Fuel Design, Irradiation Programme and Modelling, Application to Several Fuels for GENIV Systems," presentation at 2010 ATR NSUF User's Week, June 2010.

15. FY2009 Advanced Test Reactor National Scientific User Facility User's Guide, INL/EXT-08-14709, Idaho National Laboratory (2009). 
16. A. J. Palmer, G. L. McCormick, S. J. Corrigan, "Hydraulic Shuttle Irradiation System (HSIS) Recently Installed in the Advanced Test Reactor (ATR)," Proceedings of ICAPP '10, Paper 10354, San Diego, CA, USA, June 13-17, 2010

17. Future Transient Testing of Advanced Fuels - Summary of the May 4-5, 2009 Transient Testing Workshop Held at Idaho National Laboratory, INL/EXT-09-16392, September 2009.

18. http://www.iaea.org/worldatom/rrdb/, International Atomic Energy Agency, "Nuclear Research Reactors in the World", accessed April 30, 2009.

19. “Spallation Neutron Source," http://neutrons.ornl.gov/facilities/SNS/, accessed 21 May 2010.

20. T. Hill, "INSIGHTS - Intense Neutron Spectra with Independent Gamma, Hydraulic and Temperature Separate-effects for Advanced Nuclear Fuel Research and Development," unpublished draft report, September 2009.

21. Standard Guide for Use of Melt Wire Temperature Monitors for Reactor Vessel Surveillance, E706 (IIE), ASTM E 1214-06, dated February 13, 2006.

22. L L. Snead, A. M. Williams, and A. L. Qualls, "Revisiting the use of SiC as a Post Irradiation Temperature Monitor," Effects of Radiation on Materials, ASTM STP 1447, M L. Grossbeck, Ed, ASTM International, West Conshohocken, PA, 2003.

23. J. L. Rempe, K. G. Condie, D. L. Knudson, and L. L. Snead, "Comparison Measurements of Silicon Carbide Temperature Monitors,” IEEE Transactions on Nuclear Science, 57, No. 3, June 2010.

24. J. L. Rempe, D. L. Knudson, K. G. Condie, and S. C. Wilkins, "Thermocouples for High-Temperature In-Pile Testing,” Nuclear Technology, 156, No. 3, December 2006, pp 320-331.

25. J. L. Rempe, D. L. Knudson, K. G. Condie, S. C. Wilkins, J. C. Crepeau, and J. E. Daw, “Options Extending the Applicability of High Temperature Irradiation Resistant Thermocouples," invited paper for NURETH12 Special Edition, Nuclear Technology, 167, July 2009, pp 169-177.

26. D. L. Knudson, J. L. Rempe, K. G. Condie, S. C. Wilkins, J. E. Daw, and J. C. Crepeau, "High Temperature Irradiation Resistant Thermocouples - A Low Cost Sensor for In-Pile Testing at High Temperatures," Paper 8222, Proceeding of the 2008 International Congress on Advances in Nuclear Power Plants (ICAPP '08), Anaheim, CA, June 8-12, 2008.

27. J. E. Daw, J. L. Rempe, D. L. Knudson, S. C. Wilkins, and J. C. Crepeau, "High Temperature Irradiation-Resistant Thermocouple Performance Improvements," ANS NPIC HMIT 2009 Topical Meeting on Nuclear Plant Instrumentation, Controls, and Human Machine Interface Technology, Knoxville, TN, April 2009.

28. J. Daw, J. Rempe, and S. C. Wilkins, "Ultrasonic Thermometry for In-Pile Temperature Detection," 7th International Topical Meeting on Nuclear Plant Instrumentation, Control, and Human Machine Interface Technologies (NPIC\&HMIT 2010), Las Vegas, NV, November 7-11, 2010.

29. M. Laurie, D. Magallon, J. Rempe, S. Wilkins, J. Pierre, C. Marquié, S. Eymery, and R. Morice, "Ultrasonic High Temperature Sensors: Past Experiments and Prospective for Future Use," Joint International Symposium on Temperature, Humidity, and Moisture and Thermal Measurements in Industry and Science, Portorož, Slovenia, May 31 - June 4, 2010. 
30. B. Fox, H. Ban, J. Rempe, D. Knudson, and J. Daw, "Development of an in-pile technique for fuel thermal conductivity measurement," Proc. ANS NPIC HMIT 2009 Topical Meeting, Knoxville, TN, April 2009.

31. B. Fox, H. Ban, J. Rempe, J. Daw, K. Condie, D. Knudson, "In-Pile Thermal Conductivity Measurement Method for Nuclear Fuels," Thermal Conductivity 29, M. A. White (Editor), DEStech Publications Inc., to be published 2010.

32. B. Fox, H. Ban, J. Rempe, J. Daw, K. Condie, and D. Knudson, "In-Pile Thermal Conductivity Measurement Method for Nuclear Fuels," 30th International Thermal Conductivity Conference and 18th International Thermal Expansion Symposium, Pittsburgh, PA, August 29-September 2, 2009.

33. I. Cohen, B. Lustman, and J. D. Eichenberg, "Measurement of the Thermal Conductivity of Metal-Clad Uranium Oxide Rods during Irradiation," Journal of Nuclear Materials, 3, No 3 (1961), pp 331-35.

34. W.N. Beck and R. J. Fousek, "Fission Gas Release and Thermal Conductivity Measurements on U-5 wt\% F Irradiated in CP-5," Trans. American Nuclear Society, 30, 528, 1978.

35. P. R. Betten, "In-Core Measurements of Uranium-5 wt\% Fissium Alloy Thermal Conductivity, Transactions of the American Nuclear Society, 50, pp. 239-240, November 1985.

36. A. L. E. F. Schleirmacher, Vher die Warmeleitungder gase, Weidemann Ann. Phys. 34, p. 625, 1988.

37. M. S. Baghe-Khandan, Y. Choi, and M. R. Okos, "Improved Line Heat Source Thermal Conductivity Probe," Journal of Food Science, 45, p 1430-1432, 1981.

38. J.J. Healy, J. J. de Groot, and J. Kestin, "The Theory of the Transient Hot-Wire Method for Measuring Thermal Conductivity," Physica, 82C, pp 392-408, 1976.

39. X-G Liang, "The Boundary Induced Error on the Measurement of Thermal Conductivity by Transient Hot Wire Method,” Measurement Science Technology, 6, pp 467-471, 1995.

40. P. Prelovsek and B. Uran, "Generalized Hot Wire Method for Thermal Conductivity Measurements," J. Phs. E: Sci. Instrum., 17, 1984

41. K. Manohar, D. W. Yarbrough, and J. R. Booth, "Measurement of Apparent Thermal Conductivity by the Thermal Probe Method," Journal of Testing and Evaluation, September 2000.

42. Standard Test Method for Determination of Thermal Conductivity of Soil and Soft Rock by Thermal Needle Probe Procedure, ASTM D 5334-05, May 27, 2008.

43. M. Ichikawa, M. Uchida, K Yanagisawa, J. Nakamura and T. Nakajima, "Irradiation Studies of JAERI's Fuel at Halden Reactor," Journal of Nuclear Science and Technology, 25 [8], pp.609-614, August 1988.

44. M. Ichikawa, T. Fujishiro and S. Kawasaki, "LWR Fuel Safety Research with Particular Emphasis on RIA/LOCA and Other Conditions," Journal of Nuclear Science and Technology, 26(1), pp. 118 125 (January 1989).

45. B. G. Kim, J. Rempe, D. Knudson, K. Condie, and B. Sencer, "An In-situ Creep Testing Capability for the Advanced Test Reactor," submitted to Nuclear Technology, MS 10-58, April 2010. 
46. B. G. Kim, J. L. Rempe, D. L. Knudson, K. G. Condie, and B. H. Sencer, "Development of an In-situ Creep Testing Capability for the Advanced Test Reactor," 7th International Topical Meeting on Nuclear Plant Instrumentation, Control, and Human Machine Interface Technologies (NPIC\&HMIT 2010), Las Vegas, NV, November 7-11, 2010.

47. D. M. Scates, J. K. Hartwell, J. B. Walter, M. W. Drigert, J. M. Harp, "Fission Product Monitoring of Triso Coated Fuel for the Advanced Gas Reactor-1 Experiment," Proceedings of the 4th International Topical Meeting on High Temperature Reactor Technology, September 28-October 1, 2008, Washington, D.C.

48. Standard Test Method for Measuring Neutron Fluence Rates by Radioactivation of Cobalt and Silver, ASTM E481-03, February 2003.

49. Photothermal Science and Techniques, D.P. Almond and P.M. Patel, Chapman \& Hall, London, 1996.

50. J. K. Partin and G. D. Lancaster, "Radiation Resistant Fiberoptic Inspection Probes," INEEL-LDRD D043 final report, 1998

51. A.N. Gurzhiev, L.K. Turchanovich, V. G. Vasil'chenko, V. A. Bogatyrjov and V. M. Mashinsky, "Radiation damage in optical fibers," Nuclear Instruments and Methods in Physics Research A, 391 (1997) 417-422.

52. V. Hagopian, "Radiation Damage of Quartz Fibers,” Nuclear Physics B (Proc. Suppl.), 78 (1999) 635-638.

53. T. Shikama, T. Kakuta, M. Narui, T. Sagwa and H. Kayano, "Optical properties in fibers during irradiation in a fission reactor," J. Nuclear Materials 225 (1995) 324-327.

54. T. Shikama, T. Kakuta, N. Shamoto, M. Naruiand T. Sagwa, "Behavior of developed radiation-resistant silica-core optical fibers under fission reactor irradiation," Fusion Engineering and Design 51-52, (2000) 179-183.

55. G. Cheymol, H. Long, J.F. Villard, B. Brichard, "High Level Gamma and Neutron Irradiation of Silica Optical Fibers in CEA OSIRIS Nuclear Reactor," IEEE Trans. Nuc. Science, 55 (2008) 2252-2258.

56. R. S. Fielder, K. Klemer, K.L. Stinson-Bagby, "High Neutron Fluence Survivability Testing of Advanced Fiber Bragg Grating Sensors," Volume 699, pp. 650-657 (2004).

57. K. L. Telschow, D.L. Cottle and R. S. Schley, INL Letter Report on LDRD, completed 2005.

58. J. Krautkramer and H. Krautkramer, Ultrasonic Testing of Materials, 4th Edition, Springer-Verlag (Berlin), 1999.

59. R. Turrell, C. Elbaum, and B.B. Chick, Ultrasonic Methods in Solid State Physics, Academic Press (New York) 1969.

60. M. Povey, Ultrasonic Techniques for Fluids Characterization, Academic Press (Boston), 1997.

61. L.C. Lynworth, Ultrasonic Measurements for Process Control. Academic Press, Boston, 1989. 
62. V. Roque, D. Baron, J. Bourgoin, and J. M. Saurel, "Study by Acoustic Microscopy of Irradiated and Non-irradiated Uranium Dioxide, J. Nuclear Materials, 275, 305-311, 1999.

63. M. L. Batzle, D.-H. Han, and R. Hofmann, Fluid mobility and frequency-dependent seismic velocity - direct measurements. Geophysics 71 (1) N1-N9, 2006.

64. B. P. Bonner, P.A. Berge, S. C. Carlson, D. L. Farber, and J. Akella, Nanosecond Ultrasonics to Study Phase Transitions in Solid and Liquid Systems at High Pressure and Temperature, LANL, Report \# UCRL-TR-229901, April 13, 2007.

65. H. A. Spetzler, G. Chen, S. Whitehead, and I. C. Getting, "A New Ultrasonic Interferometer for the Determination of Equation of State Parameters of Sub-Millimeter Single Crystals," PAGEOPH, Vol 141, No 2/3/4, 341-377, 1993.

66. R. A. Pappas, L.J. Bond, M.S. Greenwood, and C. J. Hostick, "On-line Physical Property Process Measurements for Nuclear Fuel Recycling, "Proceedings ANS, GLOBAL 2007, Boise, ID, Sept 9-13, 2007, pp 1808-1816.

67. J. R. Bontha, H. E. Adkins, Jr, K. M. Denslow, J. W. J Jenks, C. A. Burns, P. P. Schonewill, G. P. Morgen, M. S. Greenwood, J. Blanchard, T. J. Peters, P. J. MacFarlan, E. B. K Baer, and W. A. Wilcox. Supplemental Information for Test Loop Demonstration and Evaluation of Slurry Transfer Line Critical Velocity Measurement Instruments, PNNL-19560, Pacific Northwest National Laboratory, Richland, WA, 2010.

68. D. Fourmentel, J.-F. Villard, J. Y. Ferrandis, F. Augereau, E. Rosenkrantz, and M. Dierckx, “Acoustic Sensor for In-pile Fuel Rod Fission Gas Release Measurement," Advancements in Nuclear Instrumentation Measurement Methods and their Applications (ANIMMA), 2009 First International Conference. IEEE 7-10 Marseille, France, Paper \# 978-1-4244-5207-1, June 2009.

69. L. J. Bond, S. Doctor, K. Bunch, M. Good, M. and A. E. Waltar, "Instrumentation, monitoring and NDE for Fast Reactors," Proceedings ANS, GLOBAL 2007, Boise, ID, Sept 9-13, 2007, pp 1274-1279.

70. D. Ensminger and L. J. Bond, Ultrasonics: Fundamentals, Technologies And Applications, 3rd Ed. Taylor and Frances/CRC Press [In Press], 2011.

71. P.J. Shull, Nondestructive Evaluation., Marcel Dekker, New York, 2002.

72. R. Dukes, and E.A Culpan E.A. 'Acoustic Emission: Is Techniques and Applications," IEEE Proceedings, Vol 131 Part A, No 4, June 1984, pp 241-251.

73. F.-K. Chang, Ed. "Structural Health Monitoring", Proc. 4th Int. Workshop on Structural Health Monitoring, DEStech Pubs., Lancaster, PA, Stanford University (2003).

74. M. J. Guers, and B. R. Tittmann, "Finite element analysis of ultrasonic methods for in-situ monitoring of an ASTM creep specimen," Review of Progress in Quantitative Nondestructive Evaluation, Vol. 28, 1394-1401. (2009) CP1096.

75. M. Bohenick, E. Blickley, B. R. Tittmann, and M. Kropf, "Investigating a stepped ultrasonic phased array transducer for the evaluation and characterization of defects," Proc. of SPIE, Vol. 6532 653215-1. (2007). 
76. D. A. Parks and B. R. Tittmann, "Stepped ultrasonic array for inspection of submersed plates in a harsh environment. Review of Progress in Quantitative Nondestructive Evaluation," Vol. 28, 1743-1750. (2009) CP1096.

77. A. E. Arave and R. H. Meservey, A High Temperature Ultrasonic Thermometer for Measuring Reactor Fuel Temperature, Report IN-1413, Idaho Nuclear Corp. Idaho Falls, ID, 1970.

78. A. E. Arave, High-temperature Ultrasonic Thermometer In-reactor Fuel Rod Centerline Temperature Test Results, Report ANCR-1091, Aerojet Nuclear Co. Idaho Falls, ID, 1972.

79. J. F. Bell, A. A. Fathimani, and T.N. Seth, T. N. "An ultrasonic thermometer using a resonant sensor," Int. Colloq. On high temperature in-pile thermometry, JRC Petten, The Netherlands (UKAEA and CEC) 1975 Report \# EUR 5395.

80. D. Iracane and J.-F. Villard, "Advanced Instrumentation for irradiation experiments in material testing reactors," Proceedings of the Advancements in Nuclear Instrumentation Measurement Methods and their Applications (ANIMMA), 2009 First International Conference, IEEE 7-10 June 2009, Marseille, Paper \# 978-1-4244-5208-8.

81. J.-F. Villard, and M. Schyns, "Innovations for in-pile measurements in the framework of the CEA-SCK. Joint Instrument Laboratory," Advancements in Nuclear Instrumentation Measurement Methods and their Applications (ANIMMA), 2009 First International Conference, IEEE 7-10 June 2009, Marseille, Paper \# 978-1-4244-5207-1; also published in IEEE Trans. Nuclear Sci. 57 (6) Part 2, pp 3647-3654. 2010.

82. S. Ahmed, et al., Innovative low-cost approaches to automated QA/QC of fuel particle production using on-line nondestructive methods for high reliability, Final Project Report: NERI Project 02-103: PNNL Report \# 15668, 2006.

83. R. N. Ord, and R. W. Smith, Development of an under-sodium ultrasonic scanner for in-reactor surveillance., Hanford Engineering Development Laboratory (HEDL) Report \# HEDL-TME 72-91, 1972.

84. J. W. Griffin, et al., Under-Sodium viewing: a review of ultrasonic imaging technology for liquid metal fast reactors, PNNL Report \# 18292, March 2009.

85. R. Kazys, A. Voleisis, R. Sliteris, L. Mazeika, R.V. Nieuwenhove, P. Kupschus, and H.A. Abderrahim. High temperature ultrasonic transducers for imaging and measurements in a liquid $\mathrm{Pb} / \mathrm{Bi}$ eutectic alloy. IEEE Transactions of Ultrasonics Ferroelectrics, and Frequency Control, 52(4), 525-537 (2005).

86. E. Dreacher-Krasicka, H. T. Yolken, W. C. Carter and D. de Fontaine, "Ultrasonic monitoring of phase transformations during processing of $\mathrm{YBa} 2 \mathrm{Cu} 3 \mathrm{Oz}$," In Review of Progress in QNDE, Vol 11; Thompson, D. O., Chimenti, D. E., Eds., Plenum (New York) 1837 - 1844, 1992.

87. B. Butler, S.B. Palmer, G.J. and Primavesi, "Techniques for the generation of ultrasound for extended periods at high temperatures," Ultrasonics, Vol 17 (5) 249-255, 1979.

88. J. W. Griffin, et al., Design and Demonstration of a prototype ultrasonic viewing system for liquid metal fast reactors, PNNL Report \# 19234, March 2010. 
89. Y. Soong, A.G. Blackwell, R. R. Schehl, M. F. Zarochak, and J. A. Rayne, "Ultrasonic Characterization of Three-Phase Slurries, Chem. Eng. Commun., 138, 213-224, 1995.

90. Y. Soong, I. K. Gamwo, A. G. Blackwell, F. W. Harke, R. R. Schehl and M. F. Zarochak, "Ultrasonic Characterization of Slurries in an Autoclave Reactor at Elevated Temperature," Ind. Eng. Chem. Res. 35 (6) 1807-1812 (1996).

91. G. H. Broomfield, The Effects of Temperature and Irradiation on Piezoelectric Acoustic Transducers and Materials, UKAEA Report AERE-R 11942, 1985.

92. K. E. Holbert, S. Sankaranarayanan, and S.S. McCready, "Response of Lead Metaniobate Acoustic Emission Sensors to Gamma Irradiation," IEEE Transactions on Nuclear Science 52(6), 2583-2590 (2005).

93. R. M. Severson, An experimental design for measuring in-situ radiation damage to a piezoelectric transducer, Wright Patterson, AFB Report \# AFIT/GNE/ENP/04-06, 2004.

94. F.P.J Augereau, J.-Y. Ferrandis, J.-F. Villard, D. Fourmentel, M.Dierckx, and J. Wagemans. Effects of intense neutron dose radiation on piezoceramics. Acoustics 08, 6473-6477 (2008).

95. Y.P. Meleshko, S.G. Karpechko, G.K. Leont'ev, V.I. Nalivaev, A.D. Nikiforov, and V.M. Smirnov. Radiation resistance of the piezoelectric ceramics TsTS-21 and TNV-1. Soviet Atomic Energy, 61(1), 561-564 (1986). [English translation: 1987 Plenum]

96. V. M. Baranov, S. P. Martynenko, and A. I. Sharapa. Durability of ZLT piezoceramic under the action of reactor radiation. Soviet Atomic Energy, 53(5), 803-804 (1982). [English translation: 1983 Plenum]

97. http://www.americanpiezo.com/

98. R. A. Moore, J. M. Benedetto, J.M., McGarrity, and F. B. McLean, F.B. "Neutron Irradiation Effects on PZT Thin Films for Non-volatile-memory Applications, IEEE Trans. Nuclear Sci. 38 (6) 1078-1082, 1991.

99. C.T. Searfass, B.R. Tittmann, and D.K. Agrawal, "Sol-gel Deposited Thick Film Bismuth Titanate Based Transducer Achieves Operation over $600^{\circ} \mathrm{C}$," Review of Progress in Quantitative Nondestructive Evaluation: Vol. 28, 1751-1758, 2009.

100. D.A. Parks and B.R. Tittmann, "High Temperature Broadband Contact BAW Transducer," Proceedings - IEEE Ultrasonics Symposium, 2389-2392, 2007.

101. D.A. Parks and B.R. Tittmann. "Aluminum Nitride as a High Temperature Transducer," Review of Progress in Quantitative Nondestructive Evaluation, Vol. 28, 1029-1034, 2009.

102. T. Yano, and T. Iseki. "Swelling and Microstructure of AlN Irradiated in a Fast Reactor," $J$ of Nuclear Materials vol 203, 249-254, 2003.

103. T. Shikama, T. Yano, J. Ukai, S. Onose, M. Itoh, S.J. Zinkle, L.L. Snead, T. Maruyama, S. Nagata, B. Tsuchiya, and K. Toh," Gas Nuclear Transmutation Effects in Aluminum Nitride," Fusion Science and Technology, 44, 495-499, 2003. 
104. K. Atobe, M. Okada, and M. Nakagawa, "Irradiation Temperature Dependence of Defect Formation of Nitrides (A1N and c-BN) during Neutron Irradiations," Nuclear Instruments and Methods in Physics Research B, 166-167, 57-63, 2000.

105. H. Kim, K. Park, B. Min, J.-S. Lee, K. Cho, S Kim, H.-S. Han, S.- K. Hong, and T. Yao,” Transmuted Isotopes Doped in Neutron-Irradiated ZnO Thin Films," Nuclear Instruments and Methods in Physics Research B, 217, 429-434, 2004.

106. T. Yano, K. Inokuchi, M. Shikama, J. Ukai, S. Onose, and T. Maruyama. "Neutron Irradiation Effects on Isotope Tailored Aluminum Nitride Ceramics by Fast Reactor up to $2 \times 10^{26} \mathrm{n} / \mathrm{m}^{2}, J$ of Nuclear Materials, Vol 329-333, 1471-1475, 2004.

107. T Yano, K. Ichikawa, M. Akiyoshi, and Y. Tachi, "Neutron Irradiation Damage in Aluminum Oxide and Nitride Ceramics up to a Fluence of $4.2 \times 10^{26} \mathrm{n} / \mathrm{m}^{2}, " J$ of Nuclear Materials, Vol 283-287, 947-951, 2000.

108. Bond, L.J., "Through Transmission Gas and Pulsed Water-Coupled Microscopy of Electronic Packaging and Composite Materials," August 1990-July 1992, Final Report to Materials Reliability Division, NIST, University of Colorado at Boulder, 1992.

109. M. Laurie, D. Magallon, J. Rempe, S. C. Wilkins, J. Pierre, C. Marquié, S. Eymery, R. Morice "Ultrasonic High Temperature Sensors: Past Experiments and Prospective for Future Use," Joint International Symposium on Temperature, Humidity, and Moisture and Thermal Measurements in Industry and Science, Portorož, Slovenia, May 31 - June 4, 2010.

110. M. Huger, D. Fargeot, and C. Gault, "High Temperature Measurement of Ultrasonic Wave Velocity in Refractory Materials," High Temperatures - High Pressures 43 (2) 193-201, 2000.

111. O. Bahloul, T. Chotard, M. Hauger, and C. Gault, "Young's Modulus Evolution at High Temperature of SiC Refractory Castables," J. Mater. Sci 45: 3652-3660, 2010.

112. Z. R. Martinson and R. K. McCardell, "Power-Cooling-Mismatch Test Series Test PCM-2 Test Results Report,” TREE-NUREG-1038, February 1977.

113. S. L. Sieffert, "Power-Cooling-Mismatch Test Series Test PCM-2 Test Results Report," TREE-NUREG-1069, March 1977.

114. R.J. Grossman, “A High Resolution In-Situ Ultrasonic Corrosion Monitor,” KAPL-4162, Knolls Atomic Power Laboratory, December 1, 1982.

115. M.H. November, ITT-Barton Bulletin MF-1, 1974.

116. W.H. Newman and P.P. Lele, "A Transient Heating Technique for the Measurement of Thermal Properties of Perfused Biological Tissue," Journal of Biomedical Engineering 107, pp. 219-223, 1985.

117. B.J. Davis and P.P. Lele, "An Acoustic Phase Shift Technique for Non-Invasive Measurement of Temperature Changes in Tissues,” pp. 921-924, 1985 Ultrasonics Symposium Proc., IEEE, 1985.

118. E. Rosenkrantz, J.-Y. Ferrandis, G.Leveque, and D. Baron, "Ultrasonic measurement of gas pressure and composition for nuclear fuel rods," Nuclear Instruments and Methods in Physics Research A 603, 2009. 
119. O. I. Babikov, Ultrasonics and Its Industrial Applications, Consultants Bureau, Plenum Press, 1960.

120. K. Goebbles, Materials Characterization for Process Control and Product Conformity,CRC Press, 1994.

121. M. Blaszkiewicz, "The Development of Nondestructive Evaluation (NDE) for Monitoring the Embrittlement in Nuclear Reactor Pressure Vessels," Nondestructive Characterization of Materials VII, eds. A. L. Batros, R. E. Green and C. O. Ruud (Transtec Pub. Ltd., Lebanon, New Hampshire 1996) Part 1, 9-15.

122. T. Berruti and M. M. Gola, "Acoustoelastic determination of stresses in steel using Rayleigh ultrasonic waves," Nondestructive Characterization of Materials VII, eds. A. L. Batros, R. E. Green and C. O. Ruud (Transtec Pub. Ltd., Lebanon, New Hampshire, 1996) Part 1, 171-178.

123. E. Schneider and L. Oesterlein, "Ultrasonic Characterization of Texture in Aluminum Rolled Products," Nondestructive Characterization of Materials VII, eds. A. L. Batros, R. E. Green and C. O. Ruud (Transtec Pub. Ltd., Lebanon, New Hampshire, 1996) Part 1, 405-410.

124. G. A. Ahlers, P. Purtschner, J. F. Breedis and F. N. Mandigo, "Monitoring of Texture Development in Copper-alloy Sheet," Nondestructive Characterization of Materials VIII, ed. R. E. Green (Plenum press, New York, 1998) 189-195.

125. A. Migliori, J. L. Sarrao, W. M. Visscher, T. M. Bell, M. Lei, Z. Fisk, and R. G. Leisure, "Resonant Ultrasound Spectroscopic Techniques for Measurement of the Elastic Moduli of Solids," Physica B: Condensed Matter, 183, pp. 1-24, January 1993.

126. W. M. Visscher, A. Migliori, T. M. Bell, and R. A. Reinert, "On the Normal Modes of Free Vibration of Inhomogeneous and Anisotropic Elastic Objects," Journal of the Acoustical Society of America, vol. 90, pp. 2154-62, October 1991.

127. H. Ledbetter, C. Fortunko, and P. Heyliger, "Elastic Constants and Internal Friction of polYcrystalline Copper," Journal of Materials Research, 10, pp. 1352-3, June 1995.

128. D. O. Thompson, D. K. Holmes, J. App. Phys. 27, 713, 1956.

129. A. V. Granato and K. Lücke, J. Appl. Phys. 27, 583 (1956).

130. K. Lücke and A. V. Granato, Phys. Rev. B 24, 6991 (1981).

131. H. M. Simpson and S. J. Kerkhoff, Phys. Rev. Lett. 33, 155 (1974).

132. H. M. Simpson, A. Sosin, and D.F. Johnson, Phys. Rev. B 5, 1393 (1972).

133. D. M. Parkin, J. A. Goldstone, H. M. Simpson, and J. M. Hemsky, J. Pys. F: Met. Phys. 17577 (1987).

134. J. N. Lomer and R. J. Taylor, Philosophical Magazine, Volume: 19 Issue: 159 pg 437 Published: 1969.

135. R. S. Schley, K. L. Telschow, J. B. Walter, and D. L. Cottle, "Real-time Measurement of Material Elastic Properties in a High Gamma Irradiation Environment," Nuclear Technology, 159, August 2007. 
136. S.J. Reese, K.L. Telschow, and D.H. Hurley, "On the Establishment of a Method for the Characterization of Material Microstructure through Laser Based Resonant Ultrasound Spectroscopy," Ultrasonics Ferroelectrics and Frequency Control (IEEE), 55, 770 (2008).

137. R. Hill, "The Elastic Behaviour of a Crystalline Aggregate," Proc. Physical Society of London Section A, 65349 (1952).

138. D. H. Hurley, O. B. Wright, O. Matsuda, T. Suzuki, S. Tamura, and Y. Sugawara, "Time-Resolved Surface Acoustic Wave Propagation Across a Single Grain Boundary," Physical Review B, 73 125403 (2006).

139. See for example: Gradient Lens Corporation, 207 Tremont Street, Rochester, New York 14608, www.gradientlens.com/overview_flexibles.asp, visited September 20, 2010.

140. Lenox Instrument Company, 265 Andrews Road, Trevose, PA 19053 USA, http://www.lenoxinst.com/borescopes.html, accessed September 20, 2010.

141. What is Laser Induced Breakdown Spectroscopy (LIBS)? U. S. Army Research Laboratory, Last edited September 1, 2010, http://www.arl.army.mil/www/default.cfm?Action=247\&Page=247, visited September 1, 2010.

142. "What is LIBS," Photon Machines, http://libs.photon-machines.com/?p=19.

143. David A. Cremers \& Leon J. Radziemski. Handbook of Laser-Induced Breakdown Spectroscopy (London: John Wiley \& Sons, 2006) ISBN 0470092998

144. C. Lopez-Moreno, K. Amponsah-Manager, B.W. Smith I.B. Gornushkin, N. Omenetto, and J.D. Winefordner. "Quantisation of Low-alloy Steel Samples by Powerchip Laser Induced Breakdown Spectroscopy" JAAS 2005, 20(6), 552-556.

145. H. Bette, R. Noll. "High-speed Laser-induced Breakdown Spectrometry for Scanning Microanalysis” J. Phys. D: Appl. Phys. 37, 1281-1288, 2004.

146. H. Balzer, M. Höhne, R. Noll, and V. Sturm. "New Approach to Monitoring the Al Depth Profile of Hot-dip Galvanized Sheet Steel Online using Laser-induced Breakdown Spectroscopy" Anal Bioanal Chem 2006, 385, 225-233

147. V. Sturm, L. Peter, R. Noll. "Steel Analysis with Laser-induced Breakdown Spectrometry in the vAcuum Ultraviolet" Appl. Spectroscopy 2000, 54, 1275-1278

148. H. Bao H, S.Y. Ryu, B. H. Lee, W. Tao W, and M. Gu, Nonlinear endomicroscopy using a double-clad fiber coupler, Opt Lett. 2010 Apr 1;35(7):995-7. doi: 10.1364/OL.35.000995.

149. Thomas Meek, "Semiconductive Properties of Uranium Oxides", Waste Management 2001 Symposium, Tucson, Arizona, February, 2001.

150. J. H. Rust, Nuclear Power Plant Engineering, Haralson Publishing Company: Buchanan, Georgia, 1979.

151. Idaho National Laboratory, Transactions of Presentations Given at the Fuel Cycle Research and Development Workshop, November 10, 2010, Las Vegas, Nevada. 


\section{APPENDIX A.}

\section{EXPERT WORKSHOP}




\section{A. EXPERT WORKSHOP}

As part of the process to define the FCR\&D in-pile instrumentation development program, a workshop was held on November 10, 2010 in Las Vegas, Nevada, in which international experts from fuels, irradiation, and instrumentation fields participated. During the workshop, candidate sensor technologies were discussed and ranked using agreed upon criteria. This appendix describes the technologies presented at this workshop, and the consensus reached with respect to the path forward for accomplishing the goals of this research program.

\section{A.1 Attendees}

Table A-1 lists the names and organizations of attendees participating in this workshop. As shown in this table, the meeting included nearly forty experts, that represented a wide range of domestic and international organizations, including universities, industry, and national laboratories. Attendees were selected based on their expertise in fuels behavior, irradiation testing, and instrumentation (from nuclear and non-nuclear areas).

Table A-1. Participants in FCR\&D instrumentation workshop.

\begin{tabular}{|l|l|}
\hline \multicolumn{1}{|c|}{ Name } & \multicolumn{1}{c|}{ Organization } \\
\hline Todd Allen & University of Wisconsin-Madison / ATR National Scientific User Facility \\
\hline Rick Barto & Lockheed Martin Corporation \\
\hline Kate Boudreau & Idaho National Laboratory \\
\hline Michael Bland & Lawrence Livermore National Laboratory \\
\hline Leonard Bond & Pacific Northwest National Laboratory \\
\hline Mark Bourke & Los Alamos National Laboratory \\
\hline Lori Braase & Idaho National Laboratory \\
\hline Andy Casella & Pacific Northwest National Laboratory \\
\hline Heather Chichester & Idaho National Laboratory \\
\hline Rob Daum & Electric Power Research Industry \\
\hline Joshua Daw & Idaho National Laboratory \\
\hline Bruce Hallbert & Idaho National Laboratory \\
\hline Ryan Hatcher & Lockheed Martin Corporation \\
\hline Tony Hill & Idaho National Laboratory \\
\hline Dave Hurley & Idaho National Laboratory \\
\hline Rory Kennedy & Idaho National Laboratory \\
\hline Bong Goo Kim & Korea Atomic Energy Research Institute \\
\hline Darrell Knudson & Idaho National Laboratory \\
\hline Gordon Kohse & Massachusetts Institute of Technology \\
\hline Ed Lahoda & Westinghouse \\
\hline Larry Lynnworth & Lynnworth Technical Services \\
\hline Frances Marshall & Idaho National Laboratory \\
\hline Joel McDuffee & Oak Ridge National Laboratory \\
\hline Mitch Meyer & Idaho National Laboratory \\
\hline Andy Nelson & Los Alamos National Laboratory \\
\hline Donald Olander & University of California-Berkeley \\
\hline
\end{tabular}


Table A-1. Participants in FCR\&D instrumentation workshop.

\begin{tabular}{|l|l|}
\hline \multicolumn{1}{|c|}{ Name } & \multicolumn{1}{c|}{ Organization } \\
\hline Larry Ott & Oak Ridge National Laboratory \\
\hline Ed Parma & Sandia National Laboratory \\
\hline Kemal & Idaho National Laboratory \\
\hline Joy Rempe & Idaho National Laboratory \\
\hline Gary Rochau & Sandia National Laboratory \\
\hline Rob Schley & Idaho National Laboratory \\
\hline Jim Smith & Idaho National Laboratory \\
\hline Steinar Solstad & Institute for Energy Technology/Halden Reactor Project \\
\hline Steve Taylor & Idaho National Laboratory \\
\hline T. J. Ulrich & Los Alamos National Laboratory \\
\hline Jean-Francois Villard & Commissariat à l'Energie Atomique et aux Energies Alternatives \\
\hline Bernard Wernsman & Naval Nuclear Propulsion \\
\hline S. Curtis Wilkins & Consultant \\
\hline
\end{tabular}

\section{A.2 Agenda}

To facilitate the discussion, draft versions of this report were transmitted to attendees prior to the workshop. Viewgraphs of presentations given during the workshop may be found in Reference 151. As indicated in Table A-2, most of the morning presentations highlighted background information pertaining to phenomena occurring during fuel irradiation, typical fuel irradiation conditions, and existing instrumentation to support fuel irradiations. However, most of the workshop was devoted to discussions of candidate sensor technologies that could be pursued in this FCR\&D effort. It should be noted that only a limited number of sensor technologies identified in Section 5 were presented at this workshop. Due to time constraints, a preliminary ranking was completed by INL instrumentation experts prior to the workshop (using the same criteria ultimately selected by the experts attending this workshop), and only those technologies that were ranked more highly were presented to the experts.

Table A-2. Workshop agenda.

\begin{tabular}{|c|c|c|}
\hline Time & Title & Author(s) /Presenter \\
\hline 08:00 & Welcome & J. Rempe, INL \\
\hline 08:00 & Opening/Overview of DOE-NE FCR\&D & K. Pasamehmetoglu, INL \\
\hline $08: 15$ & Changes in Fuel during Irradiation & D. Olander, UC-Berkeley \\
\hline 09:00 & $\begin{array}{l}\text { Fuel Testing Conditions /Desired Parameters for } \\
\text { Detection }\end{array}$ & H. Chichester, INL \\
\hline 09:30 & $\begin{array}{l}\text { Desired Parameters for Detection from CEA Fuel } \\
\text { Irradiations }\end{array}$ & $\begin{array}{l}\text { N. Chauvin, CEA/presented } \\
\text { by J. F. Villard, CEA }\end{array}$ \\
\hline 09:40 & $\begin{array}{l}\text { EPRI LWR fuel development and Desired Parameters for } \\
\text { Detection }\end{array}$ & R. Daum, EPRI \\
\hline $10: 00$ & Break & \\
\hline
\end{tabular}


Table A-2. Workshop agenda.

\begin{tabular}{|c|c|c|}
\hline Time & Title & Author(s) /Presenter \\
\hline $10: 15$ & $\begin{array}{l}\text { Current Instrumentation to MTR Irradiations/Candidate } \\
\text { Technology Presentation Requirements and Evaluation } \\
\text { Process }\end{array}$ & $\begin{array}{l}\text { J. L. Rempe, INL; J.-F. } \\
\text { Villard, CEA; S. Solstad, } \\
\text { IFE/HRP; B. G. Kim, } \\
\text { KAERI; and G. Kohse, MIT/ } \\
\text { presented by J. Rempe INL }\end{array}$ \\
\hline $11: 15$ & $\begin{array}{l}\text { Prospect and Development Status of Fiber Optics in } \\
\text { MTRs }\end{array}$ & $\begin{array}{l}\text { J.-F. Villard, CEA; and R. } \\
\text { Schley, INL }\end{array}$ \\
\hline $12: 00$ & Lunch & \\
\hline 13:00 & Laser Ultrasound & D Hurley, INL \\
\hline $13: 30$ & $\begin{array}{l}\text { Ultrasonic Sensing Principles and Instrumentation for } \\
\text { In-Pile Testing }\end{array}$ & $\begin{array}{l}\text { L. Lynnworth, LTS/J. Daw, } \\
\text { INL }\end{array}$ \\
\hline $14: 15$ & $\begin{array}{l}\text { Time Reversal (TR) and Nonlinear Elastic Wave } \\
\text { Spectroscopy (NEWS) for In-Situ Material Integrity } \\
\text { Diagnostics }\end{array}$ & T. J. Ulrich, LANL \\
\hline $14: 45$ & $\begin{array}{l}\text { Annular Core Research Reactor (ACRR) Transient Fuels } \\
\text { Testing and In-Pile Diagnostics Overview }\end{array}$ & $\begin{array}{l}\text { Gary Rochau, SNL; and Ed } \\
\text { Parma, SNL }\end{array}$ \\
\hline $15: 15$ & $\begin{array}{l}\text { Optical Probes, Contact Stress Sensors, Photo-acoustic } \\
\text { Gas Sensor }\end{array}$ & Mike Bland, LLNL \\
\hline $16: 00$ & Summary Discussion, Ranking, and Closeout & J. Rempe, INL \\
\hline
\end{tabular}

\section{A.3 Ranking Process}

At the end of the workshop, experts discussed the various technologies presented and provided general guidance related to the proposed research effort. In addition, experts discussed possible criteria and agreed to rank candidate technologies based on the following five items:

- Potential to detect parameters with desired accuracy. A noted objective of the FCR\&D effort is to develop instrumentation capable of unprecedented accuracy and resolution for obtaining the data needed to characterize three-dimensional changes in fuel microstructure during irradiation testing. Many of the candidate technologies offer the potential for improved detection of the "higher priority' parameters (e.g., thermal properties, cracking, porosity, etc.) identified by fuel modeling experts. However, experts agreed that there were limited sensor technologies offering the potential to directly detect changes in fuel microstructure during irradiation testing, which is the highest priority in the FCR\&D program.

- Potential to detect desired parameters in prototypic conditions (environment, temperature, etc.). Although experts recognized that some tests could be performed in non-prototypic conditions (e.g., metallic fuel surrounded by inert gas), it was agreed that such tests must be carefully planned demonstrate their applicability. Hence, sensors that can provide data in prototypic conditions were viewed more highly.

- Versatility. Section 2 identifies several parameters desired by fuel modeling experts. However, the size of irradiation tests limits the number of sensors that can be installed in a single test. Hence, 
experts ranked a single probe that can be used to detect a parameter at multiple locations more favorably. Likewise, from a funding perspective, a single technology that can provide data for detecting multiple parameters was ranked more highly because it offers the FCR\&D effort more opportunities.

- Ease of Installation. As documented in Sections 2 and 3, test rigs are complicated. The ease with which a sensor can be installed is another important consideration. Clearly, sensors are more desirable (and ranked more highly) if they can be installed without concerns about bends, breakage, special connectors, or a line of sight.

- Technology Readiness (demonstrated in-pile experience to obtained desired accuracy under desired conditions). Funding resources for the FCR\&D instrumentation development effort are limited. Technologies are less desirable if they require large investments to overcome large technology hurdles prior to deployment. Although experts deemed that all of the technologies described in Section 5 would require some investment to assess their viability for in-pile testing, experts favored technologies that could be deployed with less investment.

For this assessment, experts ranked the above criteria using the following numeric scores:

1 - Technology unable to meet most aspects of the criteria

2 - Technology may offer potential to meet some aspects of the criteria

3 - Technology may offer potential to meet most aspects of this criteria and some data available.

Experts agreed that a simpler ranking system was appropriate due to the availability of information related to technologies with respect to various criteria.

\section{A.4 Ranking Results}

Table A-3 summarizes the composite scores for the technologies presented at the workshop. Note that information presented on the ACRR was not ranked because experts indicated that the presentation focussed on ACRR capabilities rather than sensor technologies available at or for use at the ACRR. Likewise, only a limited number of sensor technologies identified in Section 5 were ranked by the experts. As noted above, a preliminary ranking was completed by INL experts prior to the workshop (using the same criteria ultimately selected by the experts attending this workshop); and only those technologies that were ranked more highly were presented to the experts.

Table A-3. Composite scores from expert evaluations.

\begin{tabular}{|l|c|}
\hline \multicolumn{1}{|c|}{ Technology } & Average Rank \\
\hline Fiber Optics & 11.2 \\
\hline Ultrasonic Techniques & 13.7 \\
\hline Laser Ultrasound & 10.5 \\
\hline NEWS \& TR & 8.1 \\
\hline Optical Probes, Contact Stress Sensors, Photo-Acoustic Gas Sensor & 5.6 \\
\hline
\end{tabular}

Discussions prior to the ranking process (and the scores reported in Table A-3) indicate that experts favored more mature technologies. Experts believed that existing research demonstrates that ultrasonic and fiber optics technologies offer the potential for earlier successes. For example, prior use of ultrasonic ther- 
mometers suggest that a single probe could be used to obtain a temperature profile with accuracies and resolutions not possible with existing technologies.

In addition, experts favored technologies that could obtain the desired data under prototypic conditions and if they offered the potential to detect most, if not all, of the parameters requested by fuel modeling experts. For example, ultrasonic sensors were ranked higher because of their potential to detect desired parameters in metallic fuel surrounded by sodium and in oxide fuel surrounded by helium for the range of temperatures of interest.

Experts also ranked technologies more highly if they offered the potential for 'diverse' parameter detection. For example, initial research investments in ultrasonic and fiber optic technologies could lead to methods that could ultimately be used to detect a wide range of parameters (e.g., physical and environmental parameters). In addition, investments in ultrasonics and fiber optic technologies offer the potential for later achieving more advanced technologies, such as laser ultrasonic, that require these technologies for successful detection and/or transmission.

Experts emphasized the need for U.S. researchers to work with organizations already possessing expertise in selected technologies. Where possible, the FCR\&D program should collaborate and/or learn from developments made by international nuclear instrumentation researchers and non-nuclear instrumentation researchers. In addition, any sensor in-pile deployment efforts should be coupled with post-irradiation examinations to maximize the insights obtained from any irradiation testing.

Experts also emphasized that sensor development efforts should simultaneously focus on capsule design and sensor viability tasks. Experts noted that having sensors installed and functioning in a viable test capsule was as important as development of the sensor. Prior to starting any research, experts emphasized the need for developing detailed plans with 'off-ramps' at key decision points where the viability of the technology could be assessed prior to further research

Last, experts emphasized the need for U.S. MTRs to gain access to instrumentation available to users at foreign test reactors and to work toward improving the accuracy of existing sensors for detecting phenomena. It was noted that many of the sensors identified in Section 4 are not currently available for use in the higher flux, harsher test conditions proposed for FCR\&D irradiations. In addition, experts noted that additional research was needed to reduce uncertainties associated with existing sensors, such as those used for flux detection.

Detailed ranking sheets and comments obtained from the experts are found in Tables A-4 through A-8. As noted above, expert comments and ranking favored mature technologies that could yield additional sensors for the FCR\&D program within the near term (e.g., less than 3 years). 
Table A-4. Detailed ranking sheet and comments for fiber optic technology.

\begin{tabular}{|c|c|c|c|c|c|}
\hline $\begin{array}{c}\text { Criterion } 1 \text { - Potential } \\
\text { to Detect Parameters } \\
\text { with Desired Accuracy }\end{array}$ & $\begin{array}{c}\text { Criterion } 2 \text { - Potential } \\
\text { to Detect in Prototypic } \\
\text { Conditions }\end{array}$ & $\begin{array}{l}\text { Criterion } 3 \\
\text {-Versatility }\end{array}$ & $\begin{array}{l}\text { Criterion } 4 \text { - } \\
\text { Installation } \\
\quad \text { Ease }\end{array}$ & $\begin{array}{l}\text { Criterion } 5 \text { - } \\
\text { Technology } \\
\text { Readiness }\end{array}$ & Comments \\
\hline \multicolumn{6}{|c|}{ Expert 1 Ranking and Comments: } \\
\hline 2.5 & 2.0 & 2.5 & 2.5 & 3.0 & 12.5 \\
\hline $\begin{array}{l}\text {-Dimensional change } \\
\text { (elongation) } \\
\text {-Temperature } \\
\text {-Pressure (indirectly) }\end{array}$ & $\begin{array}{l}\text { Needs sheath in most } \\
\text { environments but is not } \\
\text { limited if an appropriate } \\
\text { sheath can be made. } \\
\text { Temperature range to } \\
\sim 700^{\circ} \mathrm{C} \text { for fiber }\end{array}$ & & $\begin{array}{l}\text { Fragility of } \\
\text { fibers and } \\
\text { feedthrough } \\
\text { design could be } \\
\text { an issue. }\end{array}$ & $\begin{array}{l}\text { Some prototypes } \\
\text { deployed; further } \\
\text { developments in } \\
1-5 \text { years } \\
\text { expected }\end{array}$ & $\begin{array}{l}\text { Potential for multiple and } \\
\text { distributed measurements } \\
\text { is interesting but probably } \\
\text { further out in time }\end{array}$ \\
\hline \multicolumn{6}{|c|}{ Expert 2 Ranking and Comments: } \\
\hline 2.0 & 2.0 & 2.5 & 2.0 & 2.5 & 11.0 \\
\hline $\begin{array}{l}\text { Temperature, streams, } \\
\text { distance }\end{array}$ & $\begin{array}{l}\text { For environmental } \\
\text { parameters, could likely } \\
\text { operate in most } \\
\text { conditions. }\end{array}$ & & & & $\begin{array}{l}\text { Good for environmental } \\
\text { parameters, material } \\
\text { properties may be difficult. }\end{array}$ \\
\hline \multicolumn{6}{|c|}{ Expert 3 Ranking and Comments: } \\
\hline 3.0 & 1.0 & 2.5 & 1.0 & 1.0 & 8.5 \\
\hline $\begin{array}{l}\text { Thermal properties; } \\
\text { physical conditions }\end{array}$ & $\begin{array}{l}\text { Irradiation and high } \\
\text { temperatures degrade }\end{array}$ & Temperature & $\begin{array}{l}\text { Connections/ } \\
\text { sealing issues } \\
\text { must be } \\
\text { addressed }\end{array}$ & $\begin{array}{l}\text { Much more work } \\
\text { needed. }\end{array}$ & $\begin{array}{l}\text { Criteria } 2,4, \text { and } 5 \\
\text { rankings would increase if } \\
\text { temperature, irradiation, } \\
\text { and sealing issues are } \\
\text { resolved. }\end{array}$ \\
\hline \multicolumn{6}{|c|}{ Expert 4 Ranking and Comments: } \\
\hline 3.0 & 3.0 & 2.0 & 3.0 & 3.0 & $\mathbf{1 4 . 0}$ \\
\hline $\begin{array}{l}\text { Microstructure, thermal } \\
\text { properties, physical } \\
\text { conditions }\end{array}$ & $\begin{array}{l}\text { Limited temperature } \\
\text { range, radiation effect } \\
\text { on glass needs to be } \\
\text { addressed }\end{array}$ & & & $\begin{array}{l}\text { Near term } \\
\text { technology. }\end{array}$ & $\begin{array}{l}\text { Needs work on radiation } \\
\text { hardening. What causes the } \\
\text { darkening? Will } \\
\text { isotopically pure Si help? } \\
\text { Other types of glasses? } \\
\text { How about a channel } \\
\text { concept? }\end{array}$ \\
\hline \multicolumn{6}{|c|}{ Expert 5 Ranking and Comments: } \\
\hline 2.0 & 2.0 & 3.0 & 3.0 & 3.0 & 13.0 \\
\hline Physical conditions & $\begin{array}{l}\text { Maximum temperature } \\
\text { may be limited to } 700^{\circ} \mathrm{C}\end{array}$ & & & & \\
\hline \multicolumn{6}{|c|}{ Expert 6 Ranking and Comments: } \\
\hline \multicolumn{5}{|c|}{ (Expert only provided composite score) } & 13.0 \\
\hline & & & & & $\begin{array}{l}\text { In-pile in } 2011 \text { ( } \sim 1 \mathrm{yr} \\
\text { away) (BR2 Reactor, } \\
\text { Belgium) } 400^{\circ} \mathrm{C} \text { llimit a } \\
\text { concern }\end{array}$ \\
\hline \multicolumn{6}{|c|}{ Expert 7 Ranking and Comments: } \\
\hline \multirow[t]{2}{*}{3.0} & 2.0 & 3.0 & 2.0 & 1.0 & 11.0 \\
\hline & & & $\begin{array}{l}\text { Very concerned } \\
\text { about connection } \\
\text { integrity. }\end{array}$ & & \\
\hline \multicolumn{6}{|c|}{ Expert 8 Ranking and Comments: } \\
\hline & (Expert only provid & led composit & score) & & $\mathbf{1 0 . 0}$ \\
\hline
\end{tabular}


Table A-4. Detailed ranking sheet and comments for fiber optic technology.

\begin{tabular}{|c|c|c|c|c|c|}
\hline $\begin{array}{c}\text { Criterion } 1 \text { - Potential } \\
\text { to Detect Parameters } \\
\text { with Desired Accuracy }\end{array}$ & $\begin{array}{c}\text { Criterion } 2 \text { - Potential } \\
\text { to Detect in Prototypic } \\
\text { Conditions }\end{array}$ & $\begin{array}{l}\text { Criterion } 3 \\
\text {-Versatility }\end{array}$ & $\begin{array}{l}\text { Criterion } 4 \text { - } \\
\text { Installation } \\
\text { Ease }\end{array}$ & $\begin{array}{l}\text { Criterion } 5 \text { - } \\
\text { Technology } \\
\text { Readiness }\end{array}$ & Comments \\
\hline & & & & & $\begin{array}{l}\text { Concern about fiber melt } \\
\text { issue at high temperatures; } \\
\text { 2nd choice/option }\end{array}$ \\
\hline \multicolumn{6}{|c|}{ Expert 9 Ranking and Comments: } \\
\hline 3.0 & 2.0 & 3.0 & $\mathbf{3 . 0}$ & 3.0 & 14.0 \\
\hline $\begin{array}{l}\text { Thermal properties; } \\
\text { Elongation }\end{array}$ & $\begin{array}{l}700^{\circ} \mathrm{C} \text { peak temperature } \\
\text { limits use. }\end{array}$ & & & & \\
\hline \multicolumn{6}{|c|}{ Expert 10 Ranking and Comments: } \\
\hline 3.0 & $\mathbf{1 . 0}$ & 2.0 & 2.0 & 2.0 & 10.0 \\
\hline $\begin{array}{l}\text { Works well for separated } \\
\text { environmental } \\
\text { parameters. One } \\
\text { parameter at a time. }\end{array}$ & $\begin{array}{l}\text { Trouble with multiple } \\
\text { effects. }\end{array}$ & $\begin{array}{l}\text { Elongation } \\
\text { (and other } \\
\text { parameters) }\end{array}$ & & $\begin{array}{l}\text { Viable within 5+ } \\
\text { years }\end{array}$ & $\begin{array}{l}\text { Mechanical properties of } \\
\text { fiber after irradiation } \\
\text { unknown. }\end{array}$ \\
\hline \multicolumn{6}{|c|}{ Expert 11 Ranking and Comments: } \\
\hline 3.0 & 3.0 & $\mathbf{0 . 0}$ & $\mathbf{0 . 0}$ & $\mathbf{0 . 0}$ & 6.0 \\
\hline $\begin{array}{l}\text { Extensometer }(1 \mu \mathrm{m} \text { in } \\
100 \mu \mathrm{m} \text { elongation })\end{array}$ & Demonstrated in gas? & & $\begin{array}{l}\text { Demo in } 2009 \text { in } \\
\text { BR2 reactor }\end{array}$ & $\begin{array}{l}\text { Temperature } \\
\text { compensation }\end{array}$ & $\begin{array}{l}\text { Extension seems to work. } \\
\text { Other apps need R\&D. }\end{array}$ \\
\hline \multicolumn{6}{|c|}{ Expert 12 Ranking and Comments: } \\
\hline 3.0 & 2.0 & 3.0 & 2.0 & 3.0 & 13.0 \\
\hline $\begin{array}{l}\text { Thermal. Temperature: } \\
\pm<2^{\circ} \mathrm{C} \text { in primary } \\
\text { coolant circuit. }\end{array}$ & $\begin{array}{l}\text { Inert gas, water at temps } \\
\sim 1400^{\circ} \mathrm{C}\end{array}$ & $\begin{array}{l}\text { Temperature, } \\
\text { temperature } \\
\text { gradients, } \\
\text { dimensional }\end{array}$ & & $\begin{array}{l}2-3 \text { years } \\
\text { (estimated) }\end{array}$ & $\begin{array}{l}\text { Technique may be very } \\
\text { suitable for TRISO fuels } \\
\text { (carbon raman d \& g perks) }\end{array}$ \\
\hline \multicolumn{6}{|c|}{ Expert 13 Ranking and Comments: } \\
\hline 2.0 & 2.0 & 2.0 & 2.0 & 2.0 & 10.0 \\
\hline $\begin{array}{l}\text { Thermal. Enabled for } \\
\text { optical fiber } \\
\text { measurement techniques }\end{array}$ & $2000 \mathrm{psi}, 400^{\circ} \mathrm{F}$ & & & & \\
\hline
\end{tabular}


Table A-5. Detailed ranking sheet and comments for ultrasonics technology.

\begin{tabular}{|c|c|c|c|c|c|}
\hline \begin{tabular}{|c|} 
Criterion 1 - Potential \\
to Detect Parameters \\
with Desired Accuracy
\end{tabular} & \begin{tabular}{|c|} 
Criterion 2 - Potential \\
to Detect in Prototypic \\
Conditions
\end{tabular} & $\begin{array}{l}\text { Criterion } 3 \\
\text {-Versatility }\end{array}$ & $\begin{array}{l}\text { Criterion } 4 \text { - } \\
\text { Installation } \\
\text { Ease }\end{array}$ & $\begin{array}{l}\text { Criterion } 5 \text { - } \\
\text { Technology } \\
\text { Readiness }\end{array}$ & Comments \\
\hline \multicolumn{6}{|c|}{ Expert 1 Ranking and Comments: } \\
\hline 2.5 & 2.5 & 2.0 & 2.5 & 2.5 & 11.5 \\
\hline $\begin{array}{l}- \text { Temperature to } 0.1^{\circ} \mathrm{C} \\
\text {-Pressure } \\
\text {-Flow }(1 / 2 \%) . \\
\text {-Thickness }(10 \mu \mathrm{m}) \\
\text {-Gas composition } \\
\text {-Crack detection } \\
\text {-Mechanical properties }\end{array}$ & $\begin{array}{l}\text { Dependent on } \\
\text { transducers and their } \\
\text { location. Potentially } \\
\text { applicable in most } \\
\text { nuclear environments }\end{array}$ & & $\begin{array}{l}\text { For some } \\
\text { applications. }\end{array}$ & \begin{tabular}{|l|} 
Fission gas \\
composition \\
transducer already \\
deployed in \\
OSIRIS. Other \\
application 1 - \\
several years
\end{tabular} & $\begin{array}{l}\text { Potential for multiple and } \\
\text { distributed measurements } \\
\text { is interesting but probably } \\
\text { further out in time }\end{array}$ \\
\hline \multicolumn{6}{|c|}{ Expert 2 Ranking and Comments: } \\
\hline 2.5 & 3.0 & 3.0 & 2.5 & 3.0 & 14.0 \\
\hline \multicolumn{6}{|c|}{ Expert 3 Ranking and Comments: } \\
\hline 3.0 & 2.5 & 2.5 & 2.5 & 2.0 & $\mathbf{1 2 . 5}$ \\
\hline $\begin{array}{l}\text {-Temp (very accurate) } \\
\text { - Pressure } \\
\text {-Structural properties } \\
\text { (Young's, shear, etc.) } \\
\text {-Grain size } \\
\text {-Corrosion } \\
\text {-Fission gas (1\% } \\
\text { composition \& pressure) } \\
\text {-Swelling/Density }\end{array}$ & $\begin{array}{l}\text { Sodium \& inert gas, } \\
\text { temperatures are OK; } \\
\text { life of sensors concerns? }\end{array}$ & & & $\begin{array}{l}\text { Some level } \\
\text { needed, complex } \\
\text { systems, life of } \\
\text { sensors? }\end{array}$ & \\
\hline \multicolumn{6}{|c|}{ Expert 4 Ranking and Comments: } \\
\hline 3.0 & 2.0 & 3.0 & 3.0 & 3.0 & 14.0 \\
\hline $\begin{array}{l}\text {-Microstructure } \\
\text {-Level } \\
\text {-Temperature } \\
\text {-Pressure } \\
\text {-Gas composition. }\end{array}$ & & & $\begin{array}{l}\text { Good high } \\
\text { temperature } \\
\text { application }\end{array}$ & Mature & $\begin{array}{l}\text { Point measurements (like } \\
\text { grain growth) not feasible }\end{array}$ \\
\hline \multicolumn{6}{|c|}{ Expert 5 Ranking and Comments: } \\
\hline 3.0 & 2.0 & 3.0 & 2.0 & 3.0 & 13.0 \\
\hline $\begin{array}{l}\text { T, P, flow, level, density, } \\
\text { Young's Modulus, } \\
\text { corrosion }\end{array}$ & & & & & \\
\hline \multicolumn{6}{|c|}{ Expert 6 Ranking and Comments: } \\
\hline 3.0 & 3.0 & 3.0 & 3.0 & 3.0 & $\mathbf{1 5 . 0}$ \\
\hline & & & & & $\begin{array}{l}\text { Very good potential. Top } \\
\text { candidate. }\end{array}$ \\
\hline \multicolumn{6}{|c|}{ Expert 7 Ranking and Comments: } \\
\hline 3.0 & 3.0 & 3.0 & 3.0 & 3.0 & 15.0 \\
\hline $\begin{array}{l}\text { Temp, Young's Modulus, } \\
\text { Grain Growth, } \\
\text { Geometry, Corrosion }\end{array}$ & Sodium, direct coupling & & & & \\
\hline \multicolumn{6}{|c|}{ Expert 8 Ranking and Comments: } \\
\hline 2.0 & 2.0 & 2.0 & 3.0 & 3.0 & 12.0 \\
\hline
\end{tabular}


Table A-5. Detailed ranking sheet and comments for ultrasonics technology.

\begin{tabular}{|c|c|c|c|c|c|}
\hline $\begin{array}{c}\text { Criterion } 1 \text { - Potential } \\
\text { to Detect Parameters } \\
\text { with Desired Accuracy }\end{array}$ & $\begin{array}{c}\text { Criterion } 2 \text { - Potential } \\
\text { to Detect in Prototypic } \\
\text { Conditions }\end{array}$ & $\begin{array}{l}\text { Criterion } 3 \\
\text {-Versatility }\end{array}$ & $\begin{array}{l}\text { Criterion } 4 \text { - } \\
\text { Installation } \\
\text { Ease }\end{array}$ & $\begin{array}{l}\text { Criterion } 5 \text { - } \\
\text { Technology } \\
\text { Readiness }\end{array}$ & Comments \\
\hline $\begin{array}{l}\text { Separating multiple } \\
\text { effects will be fun ... }\end{array}$ & $\begin{array}{l}\text { Concern about thermal } \\
\text { contact along sensor \& } \\
\text { fuel, loss of energy in } \\
\text { sodium }\end{array}$ & & & & $\begin{array}{l}\text { Mature technology \& well } \\
\text { characterized. }\end{array}$ \\
\hline \multicolumn{6}{|c|}{ Expert 9 Ranking and Comments: } \\
\hline 3.0 & 3.0 & 3.0 & 3.0 & 3.0 & $\mathbf{1 5 . 0}$ \\
\hline $\begin{array}{l}\text { Significant - Density, } \\
\text { Grain size, Voids, } \\
\text { Evolution with time, etc. }\end{array}$ & $\begin{array}{l}\text { Needs careful selection, } \\
\text { temp issues, Na testing } \\
\text { not easy - welling } \\
\text { issues. }\end{array}$ & & $\begin{array}{l}\text { See transmission } \\
\text { as well as } \\
\text { reflection } \\
\text { configuration }\end{array}$ & $\begin{array}{l}\text { Needs demo's to } \\
\text { particular effects - } \\
\text { but mature in } \\
\text { industry wider } \\
\text { R\&D community }\end{array}$ & $\begin{array}{l}\text { A lot of more recent work } \\
\& \text { nuclear work not } \\
\text { included in review, will } \\
\text { send some info. }\end{array}$ \\
\hline \multicolumn{6}{|c|}{ Expert 10 Ranking and Comments: } \\
\hline 3.0 & 3.0 & 3.0 & 3.0 & 3.0 & 15.0 \\
\hline $\begin{array}{l}\text { Microstructure, thermal, } \\
\text { physical conditions. Gas } \\
\text { composition }( \pm 1 \%) \text {. } \\
\text { Coolant fluid temp } \\
\left( \pm 0.5^{\circ} \mathrm{C}\right) \text {, flow }\left( \pm 0.5^{\circ} \mathrm{C}\right) \text {, } \\
\text { liquid level }(<5 \mathrm{~mm}) \text {, } \\
\text { fuel temp }\left( \pm 2^{\circ} \mathrm{C}\right), \\
\text { corrosion thickness } \\
(50 \mu \mathrm{m}) \text { crack detection, } \\
\text { Young's modulus (fuel) } \\
\text { swelling/densification } \\
( \pm 1 \mu \mathrm{m})\end{array}$ & $\begin{array}{l}\text { In (cooling fluid) \& in } \\
\text { sodium to } 3000^{\circ} \mathrm{C}\end{array}$ & & & $\begin{array}{l}1-2 \text { years } \\
\text { (estimated) }\end{array}$ & \\
\hline
\end{tabular}


Table A-6. Detailed ranking sheet and comments for laser ultrasound technology.

\begin{tabular}{|c|c|c|c|c|c|}
\hline $\begin{array}{c}\text { Criterion } 1 \text { - Potential } \\
\text { to Detect Parameters } \\
\text { with Desired Accuracy }\end{array}$ & $\begin{array}{l}\text { Criterion 2 - } \\
\text { Potential to } \\
\text { Detect in } \\
\text { Prototypic } \\
\text { Conditions }\end{array}$ & $\begin{array}{l}\text { Criterion } 3 \\
\text {-Versatility }\end{array}$ & $\begin{array}{l}\text { Criterion } 4 \text { - } \\
\text { Installation } \\
\quad \text { Ease }\end{array}$ & $\begin{array}{l}\text { Criterion } 5 \text { - } \\
\text { Technology } \\
\text { Readiness }\end{array}$ & Comments \\
\hline \multicolumn{6}{|c|}{ Expert 1 Ranking and Comments: } \\
\hline 1.5 & 1.5 & 1.5 & 1.0 & 1.5 & 7.0 \\
\hline $\begin{array}{l}\text { Bulk material properties, } \\
\text {-Cracking, } \\
\text {-Microstructure. } \\
\text {-Surface (corrosion or } \\
\text { coating layers) - } 500 \mu \mathrm{m}\end{array}$ & $\begin{array}{l}\text { If } \\
\text { fiber-optic-coupled } \\
\text { has similar } \\
\text { capabilities to fiber } \\
\text { optics? Limited } \\
\text { fluence capability. }\end{array}$ & & $\begin{array}{l}\text { Not sure - need } \\
\text { work on how } \\
\text { the technique } \\
\text { can be applied } \\
\text { in-situ }\end{array}$ & \begin{tabular}{|l} 
Limited \\
demonstration in $\gamma$ \\
field and only at low \\
temperatures. \\
in-core application \\
probably several \\
years away
\end{tabular} & $\begin{array}{l}\text { May be more applicable } \\
\text { to PIE than in-core. }\end{array}$ \\
\hline \multicolumn{6}{|c|}{ Expert 2 Ranking and Comments: } \\
\hline 3.0 & 2.0 & 3.0 & 2.5 & 2.5 & 13.0 \\
\hline $\begin{array}{l}\text { Microstructure. Potential } \\
\text { to measure changes in } \\
\text { microstructure changes. }\end{array}$ & $\begin{array}{l}\text { Inert gas, possibly } \\
\text { through coolant }\end{array}$ & $\begin{array}{l}\text { Can detect } \\
\text { microstructure } \\
\text { changes }\end{array}$ & $\begin{array}{l}\text { Some issues to } \\
\text { overcome }\end{array}$ & $\begin{array}{l}\text { Will require some } \\
\text { R\&D }\end{array}$ & \\
\hline \multicolumn{6}{|c|}{ Expert 3 Ranking and Comments: } \\
\hline 3.0 & $\mathbf{1 . 0}$ & 2.0 & $\mathbf{1 . 0}$ & 1.0 & 8.0 \\
\hline $\begin{array}{l}\text { Surface structure, } \\
\text { microstructure. } \\
\text { Evolution, elastic \& } \\
\text { thermal props } \\
\text { (diffusivity) }\end{array}$ & \begin{tabular}{|l|} 
Inert Gas. Temp \& \\
irradiation \\
limitations for \\
in-reactor. \\
Fiber-optic \\
limitations
\end{tabular} & & Not yet & Years & $\begin{array}{l}\text { Need highly trained } \\
\text { technicians to } \\
\text { implement. Fiber optics } \\
\text { may limit viability. The } \\
\text { '1' rankings could go to } \\
\text { '2.5 - 3' if the temp limit/ } \\
\text { etc can be demonstrated } \\
\text { to not to be a problem }\end{array}$ \\
\hline \multicolumn{6}{|c|}{ Expert 4 Ranking and Comments: } \\
\hline 3.0 & 2.0 & 3.0 & 2.0 & 3.0 & 13.0 \\
\hline $\begin{array}{l}\text { Microstructure, Physical } \\
\text { conditions }\end{array}$ & & & $\begin{array}{l}\text { Via fiber } \\
\text { optics, fibers } \\
\text { are pacing } \\
\text { technology }\end{array}$ & & $\begin{array}{l}\text { Signal can be delivered } \\
\text { through fiber optics. }\end{array}$ \\
\hline \multicolumn{6}{|c|}{ Expert 5 Ranking and Comments: } \\
\hline 2.0 & 2.0 & 3.0 & 2.0 & 3.0 & 12.0 \\
\hline $\begin{array}{l}\text { Basic properties were not } \\
\text { addressed ie. Temp, } \\
\text { press, etc. }\end{array}$ & $\begin{array}{l}\text { Requires line of } \\
\text { sight }\end{array}$ & & & & \\
\hline \multicolumn{6}{|c|}{ Expert 6 Ranking and Comments: } \\
\hline 3.0 & 2.0 & 3.0 & 2.0 & 1.0 & 11.0 \\
\hline $\begin{array}{l}\text { Elastic Properties, some } \\
\text { plastic, thermal } \\
\text { diffusivity }\end{array}$ & $\begin{array}{l}\text { Inert gas. Limited } \\
\text { by fiber optics? }\end{array}$ & & & & \\
\hline \multicolumn{6}{|c|}{ Expert 7 Ranking and Comments: } \\
\hline 1.0 & 1.0 & 1.0 & 1.0 & $\mathbf{1 . 0}$ & 5.0 \\
\hline & & & & & Too far away \\
\hline \multicolumn{6}{|c|}{ Expert 8 Ranking and Comments: } \\
\hline 3.0 & 2.0 & 3.0 & 2.0 & 2.0 & 12.0 \\
\hline
\end{tabular}


Table A-6. Detailed ranking sheet and comments for laser ultrasound technology.

\begin{tabular}{|c|c|c|c|c|c|}
\hline $\begin{array}{c}\text { Criterion } 1 \text { - Potential } \\
\text { to Detect Parameters } \\
\text { with Desired Accuracy }\end{array}$ & $\begin{array}{l}\text { Criterion } 2 \text { - } \\
\text { Potential to } \\
\text { Detect in } \\
\text { Prototypic } \\
\text { Conditions }\end{array}$ & $\begin{array}{l}\text { Criterion } 3 \\
\text {-Versatility }\end{array}$ & $\begin{array}{c}\text { Criterion } 4 \text { - } \\
\text { Installation } \\
\text { Ease }\end{array}$ & $\begin{array}{l}\text { Criterion } 5 \text { - } \\
\text { Technology } \\
\text { Readiness }\end{array}$ & Comments \\
\hline Thermal properties & $\begin{array}{l}\text { Only prototypical } \\
\text { conditions }\end{array}$ & $\begin{array}{l}\text { Elasticity, } \\
\text { temperature }\end{array}$ & $\begin{array}{l}\text { Resolve } \\
\text { temperature } \\
\text { rating issues }\end{array}$ & & \\
\hline \multicolumn{6}{|c|}{ Expert 9 Ranking and Comments: } \\
\hline 3.0 & 1.5 & 2.0 & 2.0 & 2.0 & 10.5 \\
\hline $\begin{array}{l}\text { Indicate changes in } \\
\text { microstructure }\end{array}$ & $\begin{array}{l}\text { Robust against } \\
\text { multiple effects. } \\
\text { Environmental } \\
\text { effects. }\end{array}$ & & Not sure & $5-7$ years & $\begin{array}{l}\text { Similar to fiber optics } \\
\text { strengths and weaknesses }\end{array}$ \\
\hline \multicolumn{6}{|c|}{ Expert 10 Ranking and Comments: } \\
\hline 3.0 & 2.0 & 2.0 & 2.0 & 2.0 & 11.0 \\
\hline $\begin{array}{l}\text { UT - can detect some } \\
\text { phenomena - laser - UT } \\
\text { has deployment issues }\end{array}$ & Needs gas & $\begin{array}{l}\text { Data interpretation } \\
\text { needs more thought } \\
\text { - in particular re } \\
\text { issues like void \& } \\
\text { cracks \& } \\
\text { microstructure in } \\
\mathrm{UO}_{2} \text { fuel. }\end{array}$ & $\begin{array}{l}\text { Deployment } \\
\text { challenges. }\end{array}$ & $\begin{array}{l}\text { Needs work but } \\
\text { should have useful } \\
\text { potential }\end{array}$ & $\begin{array}{l}\text { Good for lab \& some } \\
\text { field measurements - in } \\
\text { reactor will be hard }\end{array}$ \\
\hline \multicolumn{6}{|c|}{ Expert 11 Ranking and Comments: } \\
\hline 3.0 & 2.0 & 3.0 & 2.0 & 3.0 & 13.0 \\
\hline $\begin{array}{l}\text { Microstructure } \\
\text { orientation distribution } \\
\text { function. Elastic } \\
\text { constraints. Thermal } \\
\text { constraints }\end{array}$ & $\begin{array}{l}\text { Inert gas. Temps - } \\
\text { up to } 500^{\circ} \mathrm{C}\end{array}$ & $\begin{array}{l}\text { Microstructure \& } \\
\text { thermal diffusivity }\end{array}$ & Possibly & $\begin{array}{l}2-3 \text { years } \\
\text { (estimated) }\end{array}$ & $\begin{array}{l}\text { Technology looks very } \\
\text { promising for } \\
\text { microstructural } \\
\text { monitoring real-time }\end{array}$ \\
\hline
\end{tabular}


Table A-7. Detailed ranking sheet and comments for TR \& NEWS technology.

\begin{tabular}{|c|c|c|c|c|c|}
\hline $\begin{array}{c}\text { Criterion } 1 \text { - Potential } \\
\text { to Detect Parameters } \\
\text { with Desired Accuracy }\end{array}$ & $\begin{array}{c}\text { Criterion 2 - } \\
\text { Potential to Detect in } \\
\text { Prototypic } \\
\text { Conditions }\end{array}$ & $\begin{array}{l}\text { Criterion } 3 \\
\text {-Versatility }\end{array}$ & $\begin{array}{l}\text { Criterion } 4 \text { - } \\
\text { Installation } \\
\text { Ease }\end{array}$ & $\begin{array}{l}\text { Criterion } 5 \text { - } \\
\text { Technology } \\
\text { Readiness }\end{array}$ & Comments \\
\hline \multicolumn{6}{|c|}{ Expert 1 Ranking and Comments: } \\
\hline 1.5 & 1.5 & 1.5 & 1.0 & 1.0 & 6.5 \\
\hline $\begin{array}{l}\text { Bulk material } \\
\text { characteristics }\end{array}$ & Not clear & & Not clear & $\begin{array}{l}\text { At least several } \\
\text { years for in-core } \\
\text { applications }\end{array}$ & $\begin{array}{l}\text { Seems to need a lot } \\
\text { of the same } \\
\text { development on laser } \\
\text { ultrasound for } \\
\text { practical application } \\
\text { in-core }\end{array}$ \\
\hline \multicolumn{6}{|c|}{ Expert 2 Ranking and Comments: } \\
\hline 2.5 & 2.0 & 2.5 & 2.0 & 2.0 & 11.0 \\
\hline \multicolumn{6}{|c|}{ Expert 3 Ranking and Comments: } \\
\hline \multirow[t]{2}{*}{2.5} & 2.0 & 3.0 & 2.5 & 2.0 & 12.0 \\
\hline & & & & & $\begin{array}{l}\text { Very interesting } \\
\text { technique. }\end{array}$ \\
\hline \multicolumn{6}{|c|}{ Expert 4 Ranking and Comments: } \\
\hline 1.0 & 1.0 & 1.0 & 0.5 & 0.5 & 4.0 \\
\hline $\begin{array}{l}\text { Characterization of fuel? } \\
\text { Damages (cracks)? }\end{array}$ & Radiation? Temp? & & & & \\
\hline \multicolumn{6}{|c|}{ Expert 5 Ranking and Comments: } \\
\hline 3.0 & 2.0 & 2.0 & 1.0 & 1.0 & 9.0 \\
\hline Concrete Damage & & & & & $\begin{array}{l}\text { Can it be coupled } \\
\text { with ultrasonics? } \\
\text { Could be used for } \\
\text { NDE of poolside fuel } \\
\text { (NEWS)? }\end{array}$ \\
\hline \multicolumn{6}{|c|}{ Expert 6 Ranking and Comments: } \\
\hline 2.0 & 2.0 & 2.0 & 2.0 & 2.0 & 10.0 \\
\hline $\begin{array}{l}\text { Flaw detection \& } \\
\text { material physical } \\
\text { properties }\end{array}$ & $\begin{array}{l}\text { Should work } \\
\text { anywhere ultrasonics } \\
\text { will? Piezoelectrics \& } \\
\text { laser, some apps } \\
\text { limited. }\end{array}$ & $\begin{array}{l}\text { Few parameters, } \\
\text { but parameters } \\
\text { cannot easily be } \\
\text { detected by other } \\
\text { methods }\end{array}$ & & $\begin{array}{l}\text { Needs more } \\
\text { development for } \\
\text { in-pile adaptation, } \\
\text { may be closure for } \\
\text { sodium reactors if } \\
\text { lasers are not used }\end{array}$ & $\begin{array}{l}\text { Exciting technology, } \\
\text { tremendous } \\
\text { potential, implied } \\
\text { simplicity of in-pile } \\
\text { use seems unlikely }\end{array}$ \\
\hline \multicolumn{6}{|c|}{ Expert 7 Ranking and Comments: } \\
\hline \multicolumn{5}{|c|}{ (Expert only provided composite score) } & 12.0 \\
\hline & & $\begin{array}{l}\text { Cracks versus } \\
\text { voids, if combined } \\
\text { with linear; } \\
\text { TRANS }\end{array}$ & & & \\
\hline \multicolumn{6}{|c|}{ Expert 8 Ranking and Comments: } \\
\hline 2.0 & 2.0 & 2.0 & 2.0 & 1.0 & 9.0 \\
\hline $\begin{array}{l}\text { Onset of cracking, very } \\
\text { promising for limited \# } \\
\text { parameters, very } \\
\text { uncertain for in-pile use }\end{array}$ & & & & & \\
\hline
\end{tabular}


Table A-7. Detailed ranking sheet and comments for TR \& NEWS technology.

\begin{tabular}{|c|c|c|c|c|c|}
\hline $\begin{array}{l}\text { Criterion } 1 \text { - Potential } \\
\text { to Detect Parameters } \\
\text { with Desired Accuracy }\end{array}$ & $\begin{array}{c}\text { Criterion } 2 \text { - } \\
\text { Potential to Detect in } \\
\text { Prototypic } \\
\text { Conditions }\end{array}$ & $\begin{array}{l}\text { Criterion } 3 \\
\text {-Versatility }\end{array}$ & $\begin{array}{l}\text { Criterion } 4 \text { - } \\
\text { Installation } \\
\text { Ease }\end{array}$ & $\begin{array}{c}\text { Criterion } 5 \text { - } \\
\text { Technology } \\
\text { Readiness }\end{array}$ & Comments \\
\hline \multicolumn{6}{|c|}{ Expert 9 Ranking and Comments: } \\
\hline 2.0 & 3.0 & 2.0 & 3.0 & 3.0 & 13.0 \\
\hline $\begin{array}{l}\text { Physical conditions } \\
\text { (cracks) }\end{array}$ & $\begin{array}{l}\text { Need couplant to } \\
\text { generate signal. } \\
\text { Sensor can be with a } \\
\text { laser. }\end{array}$ & & & & \\
\hline \multicolumn{6}{|c|}{ Expert 10 Ranking and Comments: } \\
\hline 1.0 & 1.0 & 1.0 & 1.0 & 1.0 & 5.0 \\
\hline $\begin{array}{l}\text { TR - Can be sensitive to } \\
\text { a number of profiles }\end{array}$ & & & $\begin{array}{l}\text { Need a } \\
\text { transmission } \\
\text { configuration. } \\
\text { Needs } 4 \\
\text { transducers and } \\
\text { detectors }\end{array}$ & No standards & Needs a lot of R\&D \\
\hline \multicolumn{6}{|c|}{ Expert 11 Ranking and Comments: } \\
\hline 1.0 & 1.0 & 1.0 & 1.0 & 1.0 & 5.0 \\
\hline $\begin{array}{l}\text { NEWS - Some } \\
\text { non-linear properties }\end{array}$ & $\begin{array}{l}\text { Loading can impact } \\
\text { results }\end{array}$ & $\begin{array}{l}\text { Can see some } \\
\text { non-linear } \\
\text { parameters. Can } \\
\text { miss voids, etc. }\end{array}$ & & $\begin{array}{l}\text { No standards. Still } \\
\text { at R\&D stage }\end{array}$ & $\begin{array}{l}\text { May be of value, but } \\
\text { needs a lot of R\&D }\end{array}$ \\
\hline \multicolumn{6}{|c|}{ Expert 12 Ranking and Comments: } \\
\hline 2.0 & 2.0 & 2.0 & 2.0 & 2.0 & 10.0 \\
\hline $\begin{array}{l}\text { NEWS - Cracking of } \\
\text { pellets center void } \\
\text { microstructure, damage } \\
\text { evolution accuracy, but } \\
\text { highly sensitive linear } \\
\text { stress corrosion cracking }\end{array}$ & In-capsule & $\begin{array}{l}\text { Yes, } \\
\text { microstructure and } \\
\text { damage } \\
\text { propagation }\end{array}$ & & $\begin{array}{l}3 \text { - } 4 \text { years } \\
\text { (estimated) }\end{array}$ & \\
\hline \multicolumn{6}{|c|}{ Expert 13 Ranking and Comments: } \\
\hline 1.0 & 2.0 & 1.0 & 2.0 & 1.0 & 7.0 \\
\hline $\begin{array}{l}\text { TR - Physical conditions. } \\
\text { Mechanical structure, } \\
\text { damage }\end{array}$ & & & & $\begin{array}{l}5-6 \text { years } \\
\text { (estimated) }\end{array}$ & $\begin{array}{l}\text { Looks a bit more } \\
\text { long-range to adapt } \\
\text { to nuclear; probably } \\
\text { needs further risk } \\
\text { burn-down by LANL }\end{array}$ \\
\hline
\end{tabular}


Table A-8. Detailed Ranking sheet and comments for optical probes, contact stress sensors, photo-acoustic gas sensor technology.

\begin{tabular}{|c|c|c|c|c|c|}
\hline $\begin{array}{c}\text { Criterion } 1 \text { - Potential } \\
\text { to Detect Parameters } \\
\text { with Desired Accuracy }\end{array}$ & \begin{tabular}{|c|} 
Criterion $2-$ \\
Potential to Detect in \\
Prototypic \\
Conditions
\end{tabular} & $\begin{array}{l}\text { Criterion } 3 \\
\text {-Versatility }\end{array}$ & $\begin{array}{l}\text { Criterion } 4 \text { - } \\
\text { Installation } \\
\text { Ease }\end{array}$ & $\begin{array}{l}\text { Criterion } 5 \text { - } \\
\text { Technology } \\
\text { Readiness }\end{array}$ & Comments \\
\hline \multicolumn{6}{|c|}{ Expert 1 Ranking and Comments: } \\
\hline 1.5 & 1.0 & 1.5 & 1.0 & 1.0 & 6.0 \\
\hline $\begin{array}{l}\text { OFP - strain (ie, } \\
\text { displacement) } \\
\text { measurement } \\
\text { OGG - } 5 \text { mm gap } \pm \mu \mathrm{m}\end{array}$ & $\begin{array}{l}\text { Not clear - probably a } \\
\text { lot of radiation } \\
\text { hardening issues }\end{array}$ & & $\begin{array}{l}\text { Not clear - } \\
\text { development } \\
\text { needed }\end{array}$ & $\begin{array}{l}\text { Several years } \\
\text { required }\end{array}$ & $\begin{array}{l}\text { Generally a subject } \\
\text { of fiber optics. } \\
\text { Photo-acoustic gas } \\
\text { sensor is potentially } \\
\text { interesting because } \\
\text { of small size. }\end{array}$ \\
\hline \multicolumn{6}{|c|}{ Expert 2 Ranking and Comments: } \\
\hline \multicolumn{5}{|c|}{ (Expert only provided composite score) } & 2.0 \\
\hline $\begin{array}{l}\text { Gas species detection } \\
\text { maybe }\end{array}$ & $\begin{array}{l}\text { Temperature \& } \\
\text { irradiation limited }\end{array}$ & No & No & Years if ever & \\
\hline \multicolumn{6}{|c|}{ Expert 3 Ranking and Comments: } \\
\hline 3.0 & 3.0 & $\mathbf{1 . 0}$ & 3.0 & 2.0 & 12.0 \\
\hline $\begin{array}{l}\text { Gap gauge contact stress } \\
\text { sensor } \\
\text { Photo - Acoustic Gas } \\
\text { Sensor (PAS) }\end{array}$ & Yes for PAS & No & & & $\begin{array}{l}\text { Gas determination } \\
\text { using PAS in fuel roc } \\
\text { with time }\end{array}$ \\
\hline \multicolumn{6}{|c|}{ Expert 4 Ranking and Comments: } \\
\hline 1.0 & 1.0 & $\mathbf{0 . 0}$ & 0.0 & $\mathbf{0 . 0}$ & 2.0 \\
\hline $\begin{array}{l}\text { Gauge-gap etc., OFP, } \\
\text { OGG, CSS, PAS, etc. }\end{array}$ & & $\begin{array}{l}\text { May have some } \\
\text { potential }\end{array}$ & No & $\begin{array}{l}\text { Long way from } \\
\text { deployment }\end{array}$ & $\begin{array}{l}\text { Technologies at } \\
\text { R\&D stage }\end{array}$ \\
\hline \multicolumn{6}{|c|}{ Expert 5 Ranking and Comments: } \\
\hline 2.0 & $\mathbf{1 . 0}$ & $\mathbf{1 . 0}$ & $\mathbf{1 . 0}$ & 1.0 & 6.0 \\
\hline $\begin{array}{l}\text { Gas composition (ppm), } \\
\text { cracks, corrosion, } \\
\text { temperature, dimensions, } \\
\text { Change }( \pm 1 \mu \mathrm{m})\end{array}$ & $\begin{array}{l}\text { Possibly (inert gas, } \\
\text { fluid) }\end{array}$ & $\begin{array}{l}\text { Each technology } \\
\text { appears to be } \\
\text { single use }\end{array}$ & Possibly & $\begin{array}{l}\sim 4-5 \text { years } \\
\text { (estimated) }\end{array}$ & $\begin{array}{l}\text { Compelling } \\
\text { technologies that } \\
\text { need development } \\
\text { for in-pile adaptation }\end{array}$ \\
\hline
\end{tabular}

